\title{
Fluoride-Promoted Cross-Coupling Reactions of Alkenylsilanols: Elucidation of the Mechanism through Kinetic Analysis
}

\author{
Scott E. Denmark,* Ramzi F. Sweis, and Daniel Wehrli \\ Roger Adams Laboratory, Department of Chemistry, University of Illinois, Urbana, Illinois, 61801
}

\section{SUPPORTING INFORMATION}

\section{General Experimental}

All reactions were performed in oven $\left(180^{\circ} \mathrm{C}\right)$ and/or flame dried glassware under an atmosphere of dry argon. All reaction temperatures corresponded to internal temperatures unless otherwise noted. All kinetic runs were run at $23{ }^{\circ} \mathrm{C} \pm 1{ }^{\circ} \mathrm{C}$ (ambient temperature). All the solvents used were technical grade and distilled from the indicated drying agents: dichloromethane: $\mathrm{P}_{2} \mathrm{O}_{5}$; diethyl ether and tetrahydrofuran: $\mathrm{Na}$, benzophenone; pentane, hexane: $\mathrm{CaH}_{2}$; methanol: $\mathrm{Mg}(\mathrm{OMe})_{2}$; ethyl acetate: $\mathrm{K}_{2} \mathrm{CO}_{3}$. "Brine" refers to a saturated solution of $\mathrm{NaCl}$. Bulb-to-bulb distillations were performed on a Büchi GKR-50 Kugelrohr and boiling points (bp) correspond to the uncorrected recorded air bath temperatures (ABT).

${ }^{1} \mathrm{H}$ NMR spectra and ${ }^{13} \mathrm{C}$ NMR spectra were recorded on a Varian Unity 400 (400 MHz, $\left.{ }^{1} \mathrm{H} ; 100 \mathrm{MHz},{ }^{13} \mathrm{C} ; 376 \mathrm{MHz},{ }^{19} \mathrm{~F}\right)$, Unity $500\left(500 \mathrm{MHz},{ }^{1} \mathrm{H} ; 126 \mathrm{MHz},{ }^{13} \mathrm{C} ; 470 \mathrm{MHz},{ }^{19} \mathrm{~F}\right)$, Inova $500\left(100 \mathrm{MHz},{ }^{29} \mathrm{Si}\right)$, Inova $600\left(119 \mathrm{MHz},{ }^{29} \mathrm{Si}\right)$ or Inova $750\left(149 \mathrm{MHz},{ }^{29} \mathrm{Si}\right)$ spectrometer. Spectra are referenced to residual chloroform $\left(\delta 7.26 \mathrm{ppm},{ }^{1} \mathrm{H} ; \delta 77.0 \mathrm{ppm},{ }^{13} \mathrm{C}\right)$, residual acetonitrile $\left(\delta 1.93 \mathrm{ppm},{ }^{1} \mathrm{H} ; \delta 1.30 \mathrm{ppm},{ }^{13} \mathrm{C}\right)$, tetramethylsilane $\left(\delta 0.00 \mathrm{ppm},{ }^{1} \mathrm{H},{ }^{13} \mathrm{C}\right.$,

$\left.{ }^{29} \mathrm{Si}\right)$ or $\alpha, \alpha, \alpha$-trifluorotoluene $\left(-63.7 \mathrm{ppm},{ }^{19} \mathrm{~F}\right) .{ }^{29} \mathrm{Si} \mathrm{NMR}$ samples contain $\mathrm{Cr}(\mathrm{acac})_{3}(2$ crystals). Chemical shifts are reported in ppm $(\delta)$; multiplicities are indicated by s (singlet), d (doublet), t (triplet), q (quartet), qn (quintet), sext (sextet), m (multiplet) and br (broad). Coupling constants, $J$, are reported in Hertz. Mass spectroscopy was performed by the University of Illinois Mass Spectrometer Center. Electron impact (EI) spectra were performed on a Finnigan-MAT CH-5 spectrometer. Data are reported in the form of m/z (intensity relative to base peak $=100)$. Infrared spectra $(\mathrm{IR})$ were recorded on an Mattson Galaxy 5020 
spectrophotometer. Peaks are reported in $\mathrm{cm}^{-1}$ with indicated relative intensities: s (strong, 67100\%); m (medium, 34-66\%); w (weak, 0-33\%). Elemental analyses were performed by the University of Illinois Microanalytical Service Laboratory.

Analytical thin-layer chromatography was performed on Merck silica gel plates with QF254 indicator. Visualization was accomplished with UV light and/or potassium permanganate. Methanol was of reagent grade and used as received; other solvents for chromatography and filtration were technical grade and distilled from the indicated drying agents: hexane and pentane $\left(\mathrm{CaCl}_{2}\right)$; ethyl acetate $\left(\mathrm{K}_{2} \mathrm{CO}_{3}\right)$. Column chromatography was performed using EM Science 230-400 mesh silica gel or ICN silica RP C18 (32-63 $\mu \mathrm{m})$ 60A or 230-400 mesh C18 Reverse Phase (RP) silica gel purchased from Fluka. Kugelrohr distillations were performed on a Büchi GKR-50 Kugelrohr; boiling points (bp) corresponding to uncorrected air-bath temperatures. All commercial reagents were purified by distillation or recrystalisation prior to use.

Analytical gas chromatography (GC) was performed using a Hewlett Packard 5890 Series II Gas Chromatograph fitted with a flame ionization detector. Injections were made on a Hewlett-Packard HP-5 (50 meter) or a Hewlett-Packard Ultra-2 (50 meter) capillary column. The injector temperature was $225^{\circ} \mathrm{C}$, the detector temperature was $300^{\circ} \mathrm{C}$, with a split ratio of 100:1. Retention times $\left(t_{R}\right)$ and integrated ratios were obtained using Agilent Chemstation Software. Sample injections were made using an HP 6890 Series Autosample Injector. Kinetic analysis was made by plotting conversion over time. Conversion was calculated by the relative integrations of the signals from the product and an internal standard, biphenyl. The slope of obtained from the conversion vs. time plots was calculated as the initial rate.

A $1.0 \mathrm{M}$ solution of tetra- $n$-butylammonium fluoride (TBAF) was made from the trihydrate salt provided by Fluka dissolved in dry THF and stored under nitrogen. 2-Dimethylaminoethanethiol hydrochloride, purchased from Aldrich, was dissolved in water to make a $20 \%$ solution which was used to quench the aliquots taken from the coupling reaction. 


\section{Literature Preparations}

(E)-Dimethyl-(1-heptenyl)silanol $((E)-1),{ }^{1}(E)$-diisopropyl-(1-heptenyl)silanol $((E)-2),{ }^{1}$ (E)-methyl-(1-heptenyl)siletane $((E)-\mathbf{3}),{ }^{2}(E)$-2-(1-heptenyl)thiophene $((E)-16),{ }^{1}(E)-1$-bromo-1heptene, ${ }^{3}$ and bis(dibenzylideneacetone)palladium $(0)^{4}$ were prepared by literature methods.

\section{Kinetics Experiments}

Each entry in the kinetics portion of the Table of Contents refers to a set of six (6) pages describing triplicate runs of the same experiment. Each individual run is entered on two (2) pages, the first describes the procedure in detail along with the data fit, and the second is a tabular listing of the raw data. 


\section{INDEX OF EXPERIMENTS}

PREPARATIVE

Page number

Preparation of Reference Compounds 6

Qualitative Rate Experiments 14

Spectroscopic Experiments

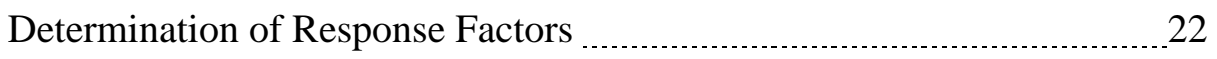

General Procedure for the Kinetic Experiments. 24

KINETICS

Page number

Order in 2-Iodothiophene

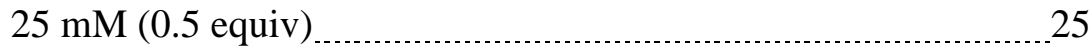

$50 \mathrm{mM}(1.0$ equiv)

$100 \mathrm{mM}(2.0$ equiv)

Order in Palladium

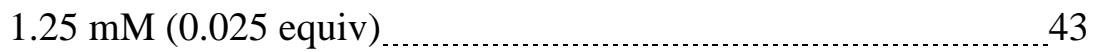

$2.5 \mathrm{mM}(0.05$ equiv $) \ldots \ldots \ldots \ldots \ldots$

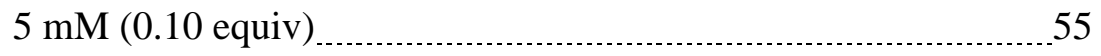

Order in Silanol $(E)-\mathbf{1}$

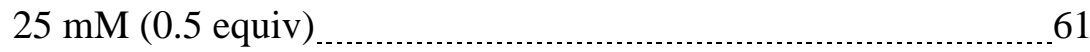

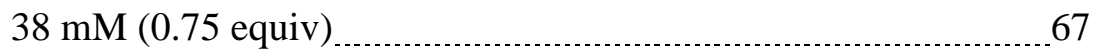

$50 \mathrm{mM}$ (1.0 equiv)

$63 \mathrm{mM}(1.25$ equiv) 79

$100 \mathrm{mM}(2.0$ equiv) 85

Order in Silanol $(E)-2$

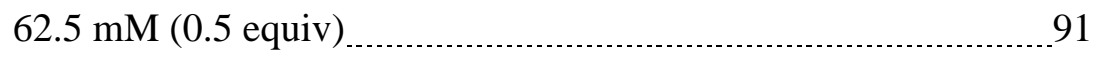

$93.8 \mathrm{mM}(0.75$ equiv $) \ldots 97$

$125 \mathrm{mM}(1.0$ equiv) 103 
$188 \mathrm{mM}$ (1.5 equiv) 109

$250 \mathrm{mM}$ (2.0 equiv)

Order in Disiloxane $(E, E)-7$

$12.5 \mathrm{mM}(0.25$ equiv $)$

$25 \mathrm{mM}(0.5$ equiv $)$

$50 \mathrm{mM}$ (1.0 equiv)

Order in TBAF (with $(E)-\mathbf{1}$ )

$50 \mathrm{mM}$ (1.0 equiv) 127

$75 \mathrm{mM}$ (1.5 equiv) 133

$100 \mathrm{mM}$ (2.0 equiv)

$150 \mathrm{mM}$ (3.0 equiv)

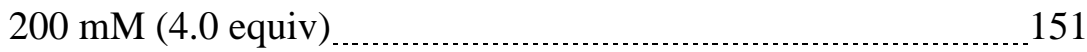

$250 \mathrm{mM}$ (5.0 equiv) 157

Order in TBAF (with (E)-2)

$125 \mathrm{mM}$ (1.0 equiv)

$250 \mathrm{mM}(2.0$ equiv) $\ldots \ldots \ldots \ldots$

$500 \mathrm{mM}$ (4.0 equiv) 175

$625 \mathrm{mM}$ (5.0 equiv) 181

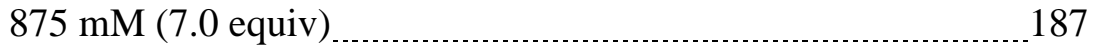

References 


\section{Reaction of $(E)$-Methyl-(1-heptenyl)silacyclobutane $(E)-3$ with TBAF [DW-V-29]}

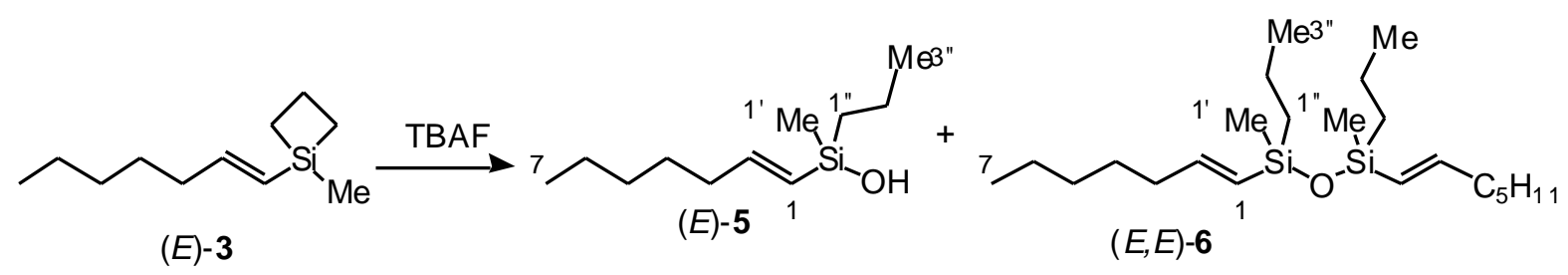

In a $10-\mathrm{mL}$, round-bottomed flask was placed $547 \mathrm{mg}(3.0 \mathrm{mmol})$ of silacyclobutane (E)-3 to which was added $3.0 \mathrm{~mL}$ (1.0 M in THF, $3.0 \mathrm{mmol}, 1.0$ equiv) of $n$-Bu $4 \mathrm{~N}^{+} \mathrm{F}^{-}$(TBAF) solution at room temperature. The solution temperature rose to $35{ }^{\circ} \mathrm{C}$ and then was allowed to cool to room temperature $(10 \mathrm{~min})$. The reaction mixture was then filtered through a plug of silica gel $(10 \mathrm{~g})$ which was further eluted with ether $(20 \mathrm{~mL})$. The combined organic fractions were dried over $\mathrm{MgSO}_{4}$, filtered and the solvent was evaporated in vacuo to give a yellow oil. This residue was purified by column chromatography (silica gel, hexane, then hexane/ethyl acetate, 19/1) to give first disiloxane $(E, E)-6$ as a colorless oil $(258 \mathrm{mg}, 45 \%$, after Kugelrohr distillation) then silanol (E)-5 also as a colorless oil (253 mg, $42 \%$, after Kugelrohr distillation).

\section{Data for $(E)-5$ :}

bp: $\quad 145^{\circ} \mathrm{C}(0.9 \mathrm{mmHg})$

${ }^{1}$ H NMR: $\left(500 \mathrm{MHz}, \mathrm{CDCl}_{3}\right)$

$6.18(\mathrm{dt}, J=18.9,6.2 \mathrm{~Hz}, \mathrm{HC}(2), 1 \mathrm{H}) ; 5.62(\mathrm{~d}, J=18.7 \mathrm{~Hz}, \mathrm{HC}(1), 1 \mathrm{H}) ; 2.11$ (m, $\mathrm{HC}(3), 2 \mathrm{H}) ; 1.60$ (brs, OH, $1 \mathrm{H}) ; 1.40(\mathrm{~m}, 4 \mathrm{H}) ; 1.29$ (m, $4 \mathrm{H}) ; 0.97$ (t, $J=7.3 \mathrm{~Hz}$, HC(3"), 3H); 0.88 (t, $J=7.1 \mathrm{~Hz}, \mathrm{HC}(7), 3 \mathrm{H}) ; 0.64$ (t, $J=6.4 \mathrm{~Hz}, \mathrm{HC}(1 "), 2 \mathrm{H}) ; 0.19$ (s, $\left.\mathrm{HC}\left(1^{\prime}\right), 3 \mathrm{H}\right)$.

${ }^{13}$ C NMR: $\left(126 \mathrm{MHz}, \mathrm{CDCl}_{3}\right)$

$149.5(\mathrm{C} 1), 129.3(\mathrm{C}(2)), 36.6(\mathrm{C}(3)), 31.4(\mathrm{C}(4)), 28.2$ (C(5)), $22.5(\mathrm{C}(6)), 19.2,18.0$, 16.6, 14.0, -1.65 (C(1')).

${ }^{29} \underline{\text { Si NMR: }}\left(99.3 \mathrm{MHz}, \mathrm{THF}-d_{8}\right)$

-0.30 .

IR: $(\mathrm{NaCl})$

3200 (s, br), 2964 (s), 2914 (s), 2874 (s), 2859 (s), 1606 (w), 1251 (s), 866 (s); 793 (s). 
$\underline{\text { MS: }}$ (EI, $70 \mathrm{eV})$

$$
200\left(\mathrm{M}^{+}, 2\right), 158 \text { (25), } 159 \text { (100), } 143 \text { (11), } 61 \text { (42). }
$$

TLC: $\quad R_{f} 0.25$ (hexane/EtOAc, 19/1).

GC: $\quad t_{R}(E)-\mathbf{4}, 5.05 \min (100 \%)\left(\mathrm{HP} 5,250{ }^{\circ} \mathrm{C}, 15 \mathrm{psi}\right)$.

Analysis: $\mathrm{C}_{11} \mathrm{H}_{24} \mathrm{OSi}$ (172.34)

$\begin{array}{lll}\text { Caculated } & \mathrm{C}, 65.93 ; & \mathrm{H}, 12.09 \% \\ \text { Found } & \mathrm{C}, 65.94 ; & \mathrm{H}, 12.30 \%\end{array}$

\section{Data for $(E, E)-6$ :}

bp: $\quad 180{ }^{\circ} \mathrm{C}(0.9 \mathrm{mmHg})$

${ }^{1} \underline{\mathrm{H} \mathrm{NMR}}:\left(500 \mathrm{MHz}, \mathrm{CDCl}_{3}\right)$

$6.09(\mathrm{dt}, J=18.6,6.4 \mathrm{~Hz}, \mathrm{HC}(2), 2 \mathrm{H}) ; 5.55$ (d, $J=18.6 \mathrm{~Hz}, \mathrm{HC}(1), 2 \mathrm{H}) ; 2.10$ (m, $\mathrm{HC}(3), 4 \mathrm{H}) ; 1.38$ (m, $16 \mathrm{H}) ; 0.95$ (t, $\left.J=7.3 \mathrm{~Hz}, \mathrm{HC}\left(3^{\prime \prime}\right), 6 \mathrm{H}\right) ; 0.89$ (t, $J=7.1 \mathrm{~Hz}, \mathrm{HC}(7)$, $6 \mathrm{H}) ; 0.56$ (t, $\left.J=5.9 \mathrm{~Hz}, \mathrm{HC}\left(1^{\prime \prime}\right), 4 \mathrm{H}\right) ; 0.08$ (s, HC(1'), $\left.6 \mathrm{H}\right)$.

${ }^{13}$ C NMR: (126 MHz, $\mathrm{CDCl}_{3}$ )

$148.4(\mathrm{C} 1), 128.6(\mathrm{C}(2)), 36.6(\mathrm{C}(3)), 31.4(\mathrm{C}(4)), 28.3(\mathrm{C}(5)), 22.5(\mathrm{C}(6)), 20.1,18.2$, 16.7, 14.0, $-0.93\left(\mathrm{C}\left(1^{\prime}\right)\right)$.

${ }^{29} \underline{\text { Si NMR: }}\left(99.3 \mathrm{MHz}, \mathrm{THF}-d_{8}\right)$

-4.25 .

IR: $(\mathrm{NaCl})$

2956 (s), 2926 (s), 2859 (s), 1054 (s), 992 (m).

$\underline{\text { MS: }}$ (EI, $70 \mathrm{eV})$

382 (M+ $\left.\mathrm{M}^{+}, 41\right), 339$ (100), 297 (17), 243 (18), 201 (24), 143 (27).

TLC: $R_{f} 0.9$ (hexane/EtOAc, 19/1).

GC: $\quad t_{R}(E)-5,9.50 \min (100 \%)\left(\mathrm{HP} 5,250{ }^{\circ} \mathrm{C}, 15 \mathrm{psi}\right)$.

Analysis: $\mathrm{C}_{22} \mathrm{H}_{46} \mathrm{OSi}_{2}(172.34)$

$\begin{array}{lll}\text { Calculated } & \text { C, 69.03; } & \text { H, } 12.11 \% \\ \text { Found } & \text { C, 68.89; } & \text { H, 12.09\% }\end{array}$




\section{Preparation of $(E, E)-1,1,7,7-T e t r a m e t h y l-1,7-d i-(1-h e p t e n y l) d i s i l o x a n e ~((E, E)-7)$ [DW-IV-62]}

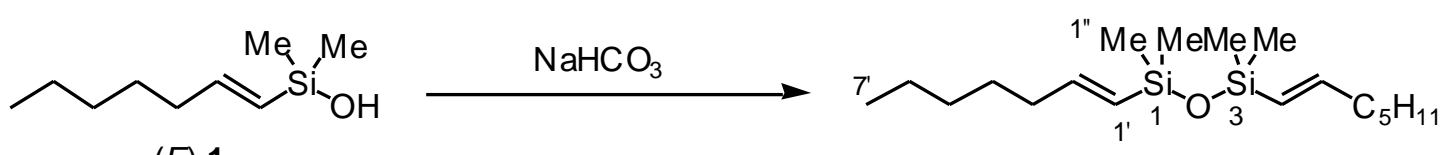

$(E)-1$

$(E, E)-7$

To a solution of $5.313 \mathrm{~g}$ (30.0 mmol) of (E)-dimethyl-(1-heptenyl)silanol (2) in $30 \mathrm{~mL}$ of ether, was added $20 \mathrm{~mL}$ of a $10 \%$ aqueous solution of $\mathrm{NaHCO}_{3}$. The reaction was stirred at room temperature for $30 \mathrm{~min}$. The aqueous phase was separated and then extracted with ether (3 $\times 10 \mathrm{~mL})$ and the combined organic phases were washed with water $(1 \times 10 \mathrm{~mL})$ and brine $(3 \times$ $30 \mathrm{~mL}$ ). The organic layer was dried over $\mathrm{MgSO}_{4}$ and filtered. The solvent was then evaporated in vacuo to give a yellow oil which was purified by distillation to afford $3.836 \mathrm{~g}(74 \%)$ of $(E, E)-7$ as a colorless oil.

Data for $(E, E)-7$ :

bp: $\quad 120{ }^{\circ} \mathrm{C}(0.9 \mathrm{mmHg})$

${ }^{1} \underline{\mathrm{H} \mathrm{NMR}}:\left(400 \mathrm{MHz}, \mathrm{CDCl}_{3}\right)$

6.09 (dt, $\left.J=18.7,6.2 \mathrm{~Hz}, \mathrm{HC}\left(2^{\prime}\right), 2 \mathrm{H}\right) ; 5.59$ (dt, $J=18.7,1.5 \mathrm{~Hz}, \mathrm{HC}\left(1^{\prime}\right), 2 \mathrm{H}$ ); 2.09 (qd, $\left.J=6.2,1.5 \mathrm{~Hz}, \mathrm{HC}\left(3^{\prime}\right), 4 \mathrm{H}\right) ; 1.39$ (qn, $\left.J=7.3 \mathrm{~Hz}, \mathrm{HC}\left(4^{\prime}\right), 4 \mathrm{H}\right) ; 1.29$ (m, HC(5') and HC(6'), 8 H); 0.89 (t, J = 7.1 Hz, HC(7'), $6 \mathrm{H}) ; 0.11$ (s, HC(1"), $12 \mathrm{H})$.

${ }^{13} \underline{\mathrm{C} \mathrm{NMR}}:\left(100.6 \mathrm{MHz}, \mathrm{CDCl}_{3}\right)$

$148.1\left(\mathrm{C}\left(1^{\prime}\right)\right), 128.2\left(\mathrm{C}\left(2^{\prime}\right)\right), 36.5\left(\mathrm{C}\left(3^{\prime}\right)\right), 31.4\left(\mathrm{C}\left(4^{\prime}\right)\right), 28.1\left(\mathrm{C}\left(5^{\prime}\right)\right), 22.5\left(\mathrm{C}\left(6^{\prime}\right)\right), 14.0$ $\left(\mathrm{C}\left(7^{\prime}\right)\right), 0.0\left(\mathrm{C}\left(1^{\prime \prime}\right)\right)$.

${ }^{29} \underline{\text { Si NMR: }}\left(99.3 \mathrm{MHz}, \mathrm{THF}-d_{8}\right)$

-3.95 .

IR: $(\mathrm{NaCl})$

2959 (s), 2928 (s), 2858 (s), 1620 (s), 1253 (s), 1052 (s), 992 (m), 841 (s); 798 (s).

$\underline{\text { MS: }}$ (EI, $70 \mathrm{eV})$

$326\left(\mathrm{M}^{+}, 16\right), 311$ (16), 213 (18), 157 (21), 133 (100), 119 (42).

TLC: $\quad R_{f} 0.91$ (pentane/EtOAc, 19/1).

Analysis: $\mathrm{C}_{18} \mathrm{H}_{38} \mathrm{OSi}_{2}(172.34)$

Calculated C, 66.18; H, 11.73; Si, 17.19\%

Found $\quad$ C, 66.20; H, 11.99; Si, $17.23 \%$ 


\section{Preparation of $(E)$-Dimethylfluoro-(1-heptenyl)silane $((E)-4)$ [DW-IV-61]}

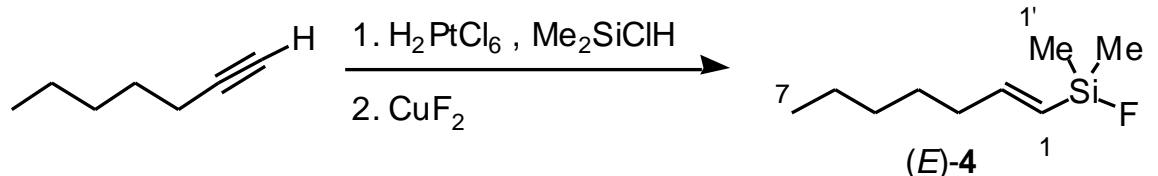

Hexachloroplatinic acid (26.6 mg, $65 \mu \mathrm{mol}, 0.005$ equiv) was dissolved in $1 \mathrm{~mL}$ of 2propanol and $30 \mathrm{~mL}$ of diethyl ether in a dry, two-neck, round-bottom flask equipped with a stir bar and a reflux condenser under an atmosphere of dry argon. Chlorodimethylsilane (2.155 g, $14.3 \mathrm{mmol}, 1.1$ equiv) was then added and the mixture was heated to reflux. A solution of 1.250 $\mathrm{g}(13.0 \mathrm{mmol})$ of 1-heptyne in $10 \mathrm{~mL}$ of dry ether was then added dropwise over $10 \mathrm{~min}$, at a rate sufficient to maintain reflux of the reaction mixture. After the addition was complete, the mixture was heated in an oil bath to reflux for $4 \mathrm{~h}$. After cooling to room temperature, the solvent was evaporated in vacuo and the residual oil was distilled $\left(114{ }^{\circ} \mathrm{C}\right.$ at $\left.6 \mathrm{mmHg}\right)$ to give $2.682 \mathrm{~g}(84 \%)$ of the chlorosilane as a colorless liquid.

The intermediate chlorosilane $(2.731 \mathrm{~g}, 11.1 \mathrm{mmol})$ was dissolved in $20 \mathrm{~mL}$ of ether and $564 \mathrm{mg}(5.55 \mathrm{mmol})$ of $\mathrm{CuF}_{2}$ was added. The mixture was stirred at room temperature overnight and was then filtered through Celite ${ }^{\circledR}$. The filtrate was dried over $\mathrm{MgSO}_{4}$, filtered and the solvents evaporated in vacuo to give a oil which was distilled twice to give $2.408 \mathrm{~g}(95.3 \%)$ of $(E)-\mathbf{4}$ as a colorless oil.

Data for $(E)-\mathbf{4}$ :

bp: $\quad 70{ }^{\circ} \mathrm{C}(20 \mathrm{mmHg})$

${ }^{1} \underline{\mathrm{H} \mathrm{NMR}}:\left(500 \mathrm{MHz}, \mathrm{CDCl}_{3}\right)$

6.27 (dtd, $J=18.7,6.2,1.1 \mathrm{~Hz}, \mathrm{HC}(2), 1 \mathrm{H}) ; 5.65$ (dtd, $J=18.7,1.5,1.5 \mathrm{~Hz}, \mathrm{HC}(1), 1$ H); 2.14 (qd, $J=7.3,1.3 \mathrm{~Hz}, \mathrm{HC}(3), 2 \mathrm{H}) ; 1.41$ (qn, $J=7.3 \mathrm{~Hz}, \mathrm{HC}(4), 2 \mathrm{H}) ; 1.31$ (m, $\mathrm{HC}(5)$ and $\mathrm{HC}(6), 4 \mathrm{H}) ; 0.89$ (t, $J=7.1 \mathrm{~Hz}, \mathrm{HC}(7), 3 \mathrm{H}) ; 0.27$ (d, $J=7.3 \mathrm{~Hz}, \mathrm{HC}\left(1^{\prime}\right), 6$ $\mathrm{H})$.

${ }^{13}$ C NMR: $\left(100 \mathrm{MHz}, \mathrm{CDCl}_{3}\right)$

$151.4(\mathrm{~d}, J=4.2 \mathrm{~Hz}, \mathrm{C}(2)), 125.2(\mathrm{~d}, J=16.8 \mathrm{~Hz}, \mathrm{C}(1)), 36.4(\mathrm{C}(3)), 31.2(\mathrm{C}(4)), 27.8$ (C(5)), $22.4(\mathrm{C}(6)), 13.9(\mathrm{C}(7)),-1.4\left(\mathrm{~d}, J=16.0 \mathrm{~Hz}, \mathrm{C}\left(1^{\prime}\right)\right)$. 
${ }^{19} \underline{\text { F NMR: }}\left(376 \mathrm{MHz}, \mathrm{CDCl}_{3}\right)$

$-160.2$

${ }^{29}$ Si NMR: (99 MHz, THF- $\left.d_{8}\right)$

$18.40(\mathrm{~d}, J=225 \mathrm{~Hz})$.

IR: $(\mathrm{NaCl})$

2929 (s), 2859 (s), 1619 (s), 1467 (s), 1256 (s), 993 (s), 871 (s).

MS: (EI, $70 \mathrm{eV})$

174 (M+, 2.6), 159 (14), 155 (23), 118 (48), 89 (11), 77 (100), 63 (11).

Analysis: $\mathrm{C}_{9} \mathrm{H}_{19} \mathrm{FOSi}(174.33)$

Calculated C, 62.01; H, 10.99; F, 10.90; Si, 16.11\%

Found $\quad$ C, 61.96; H, 11.02; $\quad$ F, 10.53; $\quad \mathrm{Si}, 16.40 \%$

Preparation of $(E, E)-1,1,7,7-T e t r a i s o p r o p y l-1,7-d i-(1-h e p t e n y l) d i s i l o x a n e ~((E, E)-8)$ [DWIX-74]

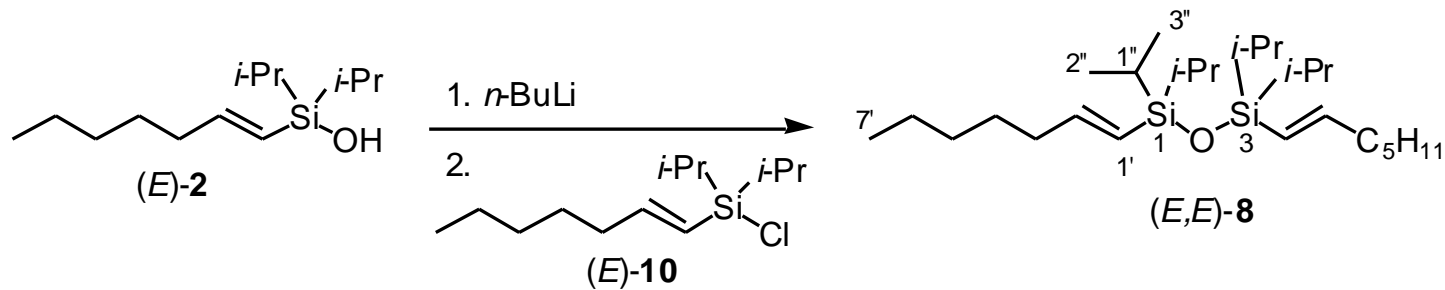

In a 250-mL, flame-dried, round-bottomed flask, $1142 \mathrm{mg}(5.0 \mathrm{mmol})$ of (E)-diisopropyl1-heptenylsilanol ${ }^{2}$ was dissolved in $50 \mathrm{~mL}$ dry THF under $\mathrm{N}_{2}$ and was cooled to $0{ }^{\circ} \mathrm{C} . n$-BuLi (3.34 mL, $1.5 \mathrm{M}, 5.0 \mathrm{mmol}$ ) was added via syringe and the reaction was stirred at $0{ }^{\circ} \mathrm{C}$ for 10 min. (E)-Diisopropyl-1-heptenylchlorosilane ${ }^{2}((E)-10)(1235 \mathrm{mg}, 5.0 \mathrm{mmol})$ was then added and the solution was warmed to $\mathrm{rt}$, at which temperature it was stirred for an additional $36 \mathrm{~h}$. The reaction mixture was then poured into ether $(20 \mathrm{~mL})$ and the mixture was washed with water $(3 \times 50 \mathrm{~mL})$ and brine $(1 \times 50 \mathrm{~mL})$. The organic phase was then dried over $\mathrm{MgSO}_{4}$, filtered and the solvent was evaporated in vacuo, to give a colorless oil. Purification of the oil by column chromatography (silica gel, pentane), followed by distillation afforded $1140 \mathrm{mg}$ (52\%) of desired $(E, \mathrm{E})-\mathbf{8}$ as a colorless oil and $800 \mathrm{mg}(35 \%)$ of starting silanol $(E)-\mathbf{2}$ as colorless oil. 
Data for $(E, E)-8$ :

bp: $\quad 120{ }^{\circ} \mathrm{C}(0.2 \mathrm{mmHg})$

${ }^{1} \underline{\mathrm{H} \mathrm{NMR}}:\left(500 \mathrm{MHz}, \mathrm{CDCl}_{3}\right)$

6.14 (dt, $\left.J=18.7,6.4 \mathrm{~Hz}, \mathrm{HC}\left(2^{\prime}\right), 2 \mathrm{H}\right) ; 5.51$ (dt, $\left.J=18.7,1.5 \mathrm{~Hz}, \mathrm{HC}\left(1^{\prime}\right), 2 \mathrm{H}\right) ; 2.11$ (qd, $\left.J=6.6,1.5 \mathrm{~Hz}, \mathrm{HC}\left(3^{\prime}\right), 4 \mathrm{H}\right) ; 1.39$ (qn, $\left.J=6.9 \mathrm{~Hz}, \mathrm{HC}\left(4^{\prime}\right), 4 \mathrm{H}\right) ; 1.30$ (m, HC(5') and $\left.\mathrm{HC}\left(6^{\prime}\right), 8 \mathrm{H}\right) ; 1.1-0.92$ (m, $\mathrm{HC}\left(1^{\prime \prime}\right), \mathrm{HC}\left(2^{\prime \prime}\right)$ and $\left.\mathrm{HC}\left(3^{\prime \prime}\right), 14 \mathrm{H}\right) ; 0.88$ (t, $J=6.8 \mathrm{~Hz}$, $\left.\mathrm{HC}\left(7^{\prime}\right), 6 \mathrm{H}\right)$.

${ }^{13} \underline{\mathrm{C} \mathrm{NMR}}:\left(125 \mathrm{MHz}, \mathrm{CDCl}_{3}\right)$

$149.6\left(\mathrm{C}\left(2^{\prime}\right)\right), 124.8\left(\mathrm{C}\left(1^{\prime}\right)\right), 36.9\left(\mathrm{C}\left(3^{\prime}\right)\right), 31.3\left(\mathrm{C}\left(4^{\prime}\right)\right), 28.4\left(\mathrm{C}\left(5^{\prime}\right)\right), 22.5\left(\mathrm{C}\left(6^{\prime}\right)\right), 17.49$, $17.43,14.0\left(\mathrm{C}\left(1^{\prime \prime}\right)\right), 13.4\left(\mathrm{C}\left(7^{\prime}\right)\right)$.

${ }^{29} \underline{\text { Si NMR: }}\left(149 \mathrm{MHz}, \mathrm{THF}-d_{8}\right)$

-3.56 .

IR: $(\mathrm{NaCl})$

2958 (s), 2927 (s), 2865 (s), 1619 (m), 1464 (m), 1083 (s), 1056 (s), 993 (s), 882 (m).

MS: (EI, $70 \mathrm{eV})$

$438\left(\mathrm{M}^{+}, 0.6\right), 395$ (100), 299 (26), 225 (77), 169 (78), 141 (21), 97 (12), 85 (23), 59 (30).

TLC: $R_{f} 0.95$ (pentane).

Analysis: $\mathrm{C}_{26} \mathrm{H}_{54} \mathrm{OSi}_{2}(438.89)$

Calculated $\quad \mathrm{C}, 71.15 ; \quad \mathrm{H}, 12.40 ; \quad \mathrm{Si}, 12.80 \%$

Found $\quad \mathrm{C}, 71.23 ; \quad \mathrm{H}, 12.53 ; \quad \mathrm{Si}, 12.76 \%$ 
Preparation of $(E, E)-1,1-D i i s o p r o p y l-7,7-d i m e t h y l-1,7-d i-(1-h e p t e n y l) d i s i l o x a n e ~((E, E)-9)$ [DW-IX-75]

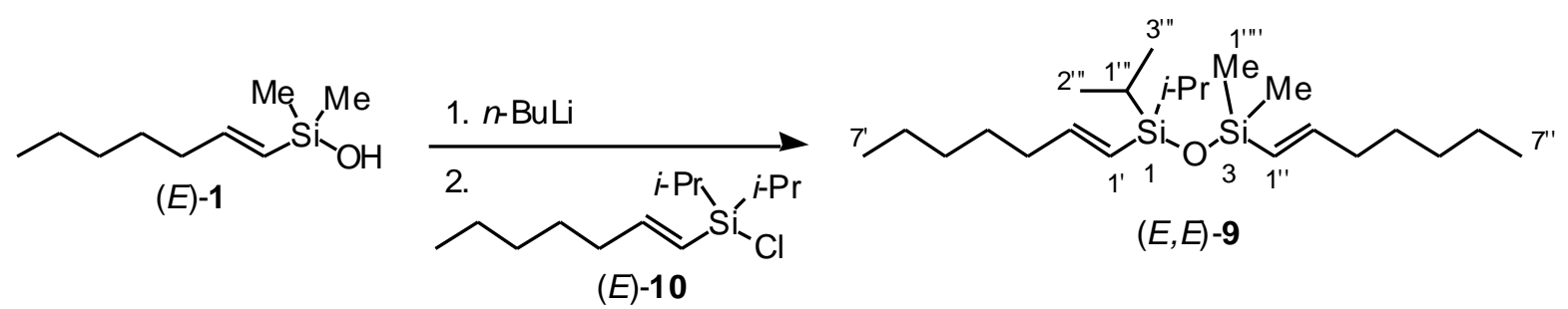

In a flame-dried, 250-mL, round-bottomed flask, was placed $862 \mathrm{mg}(5.0 \mathrm{mmol})$ of $(E)$ dimethyl-(1-heptenyl)silanol ((E)-1) together with $50 \mathrm{~mL}$ dry THF under $\mathrm{N}_{2}$ and the solution was cooled to $0^{\circ} \mathrm{C}$. $n$-BuLi $(3.34 \mathrm{~mL}, 1.5 \mathrm{M}, 5.0 \mathrm{mmol})$ was added via syringe and the solution was stirred at $0{ }^{\circ} \mathrm{C}$ for $10 \mathrm{~min}$. (E)-Diisopropyl-1-heptenylchlorosilane ${ }^{2}(1482 \mathrm{mg}, 6.0 \mathrm{mmol})$ was then added and the mixture was warmed to rt, at which temperature it was stirred for $36 \mathrm{~h}$. The reaction mixture was then poured into ether $(20 \mathrm{~mL})$ which was washed with water $(3 \times 50$ $\mathrm{mL})$ and brine $(1 \times 50 \mathrm{~mL})$. The organic phase was then dried over $\mathrm{MgSO}_{4}$, filtered and the solvent was evaporated in vacuo, to give a colorless oil. Purification by column chromatography (silica gel, pentane, then reversed phase $\mathrm{C}-18, \mathrm{MeOH} / \mathrm{H}_{2} \mathrm{O}, 19 / 1$ ), followed by distillation afforded $1192 \mathrm{mg}(62 \%)$ of $(E, E)-\mathbf{9}$ as a colorless oil.

Data for $(E, E)-9$ :

bp: $\quad 97{ }^{\circ} \mathrm{C}(0.2 \mathrm{mmHg})$

${ }^{1} \underline{\mathrm{H} \mathrm{NMR}}:\left(500 \mathrm{MHz}, \mathrm{CDCl}_{3}\right)$

$6.10\left(\mathrm{dt}, J=18.7,6.2 \mathrm{~Hz}\right.$,coinciding $\mathrm{HC}\left(2^{\prime}\right)$ and $\left.\mathrm{HC}\left(2^{\prime \prime}\right), 2 \mathrm{H}\right) ; 5.62(\mathrm{dt}, J=18.7,1.5 \mathrm{~Hz}, 1$ $\mathrm{H}) ; 5.46(\mathrm{dt}, J=18.9,1.5 \mathrm{~Hz}, 1 \mathrm{H}) ; 2.11\left(\mathrm{~m}, \mathrm{HC}\left(3^{\prime}\right)\right.$ and $\left.\mathrm{HC}\left(3^{\prime \prime}\right), 4 \mathrm{H}\right) ; 1.39$ (m, HC(4') and $\left.\mathrm{HC}\left(4^{\prime \prime}\right), 4 \mathrm{H}\right) ; 1.29$ (m, $\mathrm{HC}\left(5^{\prime}\right), \mathrm{HC}\left(5^{\prime \prime}\right), \mathrm{HC}\left(6^{\prime}\right)$ and $\left.\mathrm{HC}\left(6^{\prime \prime}\right), 8 \mathrm{H}\right) ; 0.96$ (d, J= $7.1 \mathrm{~Hz}$, $3 \mathrm{H}) ; 0.93$ (d, $J=7.1 \mathrm{~Hz}, 3 \mathrm{H}) ; 0.95$ (m, HC(1"'), $1 \mathrm{H}) ; 0.888$ (t, $J=6.6 \mathrm{~Hz}, 3 \mathrm{H}) ; 0.885$ (t, $J=7.0 \mathrm{~Hz}, 3 \mathrm{H}) ; 0.13$ (s, HC(1'"'), $6 \mathrm{H})$.

${ }^{13} \underline{\mathrm{C} \mathrm{NMR}}:\left(125 \mathrm{MHz}, \mathrm{CDCl}_{3}\right)$

$149.8,148.0,129.5,124.3,36.8,36.5,31.4,31.3,28.4,28.3,22.52,22.48,17.3,17.1$, $14.05,14.02,13.0,0.9$. 
${ }^{29} \underline{\text { Si NMR: }}\left(149 \mathrm{MHz}, \mathrm{THF}-d_{8}\right)$

$-2.30,-4.88$.

IR: $(\mathrm{NaCl})$

2927 (s), 2864 (s), 1619 (s), 1464 (s), 1252 (s), 1084 (s), 993 (s), 882 (s), 838 (s), 792 (s).

MS: (EI, $70 \mathrm{eV})$

382 (M+, 0.5), 339 (100), 299 (13), 243 (30), 225 (20), 169 (20), 105 (14), 73 (11), 59 (18).

TLC: $\quad R_{f} 0.92$ (pentane).

HRMS: calculated for $\mathrm{C}_{22} \mathrm{H}_{46} \mathrm{OSi}_{2}, 382.308723$; found, 382.3089 .

Preparation of Tetramethylammonium (E)-Dimethyl-(1-heptenyl)silyloxide ((E)-15) [DWVIII-56]

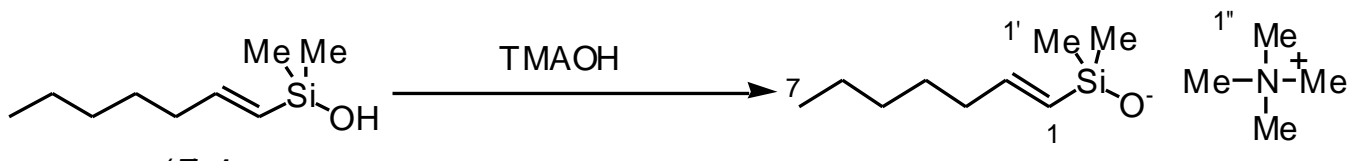

$(E)-1$

$(E)-15$

In a two-neck, 200-mL, round-bottomed flask, were placed $1035 \mathrm{mg}(6.0 \mathrm{mmol})$ of silanol (E)-1 and a solution of $1089 \mathrm{mg}(6.0 \mathrm{mmol})$ tetramethylammonium hydroxide pentahydrate in $i$-PrOH $(100 \mathrm{~mL})$ at $\mathrm{rt}$ under $\mathrm{N}_{2}$. The flask was evacuated and the solvent was removed. This process was repeated twice with $i$-PrOH $(100 \mathrm{~mL})$, twice with $\mathrm{CH}_{3} \mathrm{CN}(100 \mathrm{~mL})$ and once with methanol $(100 \mathrm{~mL})$. The oily residue was then evacuated overnight to form brownish crystals (1465 mg) which were stored in the glove-box.

Data for $(E) \mathbf{- 1 5}$ :

${ }^{1} \underline{\mathrm{H} N M R}:\left(500 \mathrm{MHz}, \mathrm{CD}_{3} \mathrm{CN}\right)$

5.89 (dt, $J=18.2,6.3 \mathrm{~Hz}, \mathrm{HC}(2), 1 \mathrm{H}) ; 5.63$ (dt, $J=18.2,1.5 \mathrm{~Hz}, \mathrm{HC}(1), 1 \mathrm{H}$ ); 3.14 (s, $\mathrm{HC}(1 "), 12 \mathrm{H}) ; 2.00$ (qd, $J=6.3,1.5 \mathrm{~Hz}, \mathrm{HC}(3), 2 \mathrm{H}) ; 1.33$ (m, HC(4), HC(5) and $\mathrm{HC}(6)$, $6 \mathrm{H}) ; 0.87$ (t, $J=6.9 \mathrm{~Hz}, \mathrm{HC}(7), 3 \mathrm{H}) ;-0.20$ (s, HC(1'), $3 \mathrm{H})$.

${ }^{13} \mathrm{C} \mathrm{NMR}:\left(126 \mathrm{MHz}, \mathrm{CD}_{3} \mathrm{CN}\right)$

$142.8(\mathrm{C}(2)), 56.0\left(\mathrm{C}\left(1^{\prime \prime}\right)\right), 37.6(\mathrm{C}(3)), 32.4(\mathrm{C}(4)), 29.7$ (C(5)), $23.3(\mathrm{C}(6)), 14.4(\mathrm{C}(7))$, $3.8\left(\mathrm{C}\left(1^{\prime}\right)\right)$. 
${ }^{29} \underline{\text { Si NMR: }}\left(119 \mathrm{MHz}, \mathrm{CD}_{3} \mathrm{CN}\right)$

-20.9 .

${ }^{29}$ Si NMR: $\left(119 \mathrm{MHz}, \mathrm{DMF}-d_{7}\right)$

-26.2 .

Analysis: $\mathrm{C}_{13} \mathrm{H}_{31} \mathrm{NOSi}(245.48)$

$\begin{array}{llll}\text { Calculated } & \mathrm{C}, \text { 63.61; } & \mathrm{H}, 12.73 ; & \mathrm{N}, 5.71 \% \\ \text { Calculated for 1.5 } \mathrm{H}_{2} \mathrm{O} & \mathrm{C}, 57.68 ; & \mathrm{H}, 12.59 ; & \mathrm{N}, 5.17 \% \\ \text { Found } & \mathrm{C}, 57.73 ; & \mathrm{H}, 12.78 ; & \mathrm{N}, 5.15 \%\end{array}$

Qualitative Rate Experiments Scheme 3. General Procedure.

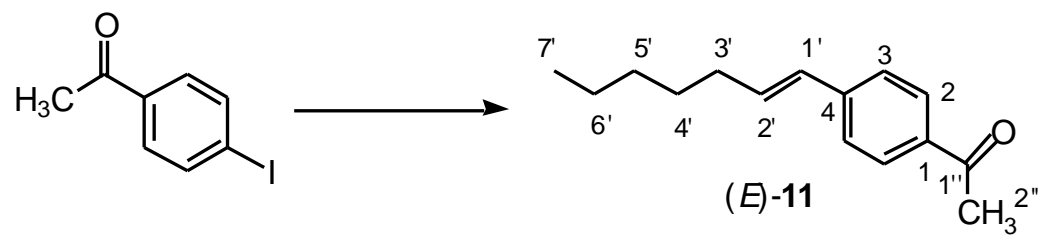

The organosilicon compound (silanol and fluorosilane $1.0 \mathrm{mmol}$, disiloxane $0.5 \mathrm{mmol}$ ) was added to a solution of the indicated activator (0-2 equiv) in THF and the mixture was stirred for $10 \mathrm{~min}$ at rt. 4-Iodoacetophenone ( $246 \mathrm{mg}, 1.0 \mathrm{mmol}$ ) was added to the mixture, followed by the palladium catalyst $(29 \mathrm{mg}, 5 \mathrm{~mol} \%)$ and the mixture was stirred at room temperature for 10 min-24 h. The reaction mixture was then filtered through a short silica gel column $(20 \mathrm{~g})$. The plug was washed with diethyl ether $(100 \mathrm{~mL})$ and the solvent was evaporated in vacuo. The residue was purified by column chromatography (Reverse Phase $\mathrm{C} 18, \mathrm{MeOH}-\mathrm{H}_{2} \mathrm{O}$ 4/1) to afford the coupling product $(E)$-11 which was further purified by distillation.

\section{Reaction of 4-Iodoacetophenone with $(E)$-Dimethyl-(1-heptenyl)silanol $((E)-1)$ in the Presence of Tetra- $\boldsymbol{n}$-butylammonium Fluoride ( 2 equiv) [DW-IV-92].}

Following the General Procedure, $(E)-1(172 \mathrm{mg}, 1.0 \mathrm{mmol})$ was dissolved in TBAF solution (2 mL, 1.0 M in THF, $2.0 \mathrm{mmol})$ and THF $(1 \mathrm{~mL})$ and was stirred for $10 \mathrm{~min}$ at rt. 4Iodoacetophenone (246 mg, $1.0 \mathrm{mmol})$ and $\mathrm{Pd}(\mathrm{dba})_{2}(29 \mathrm{mg}, 0.05$ equiv) were added and the mixture was stirred at $\mathrm{rt}$ for $10 \mathrm{~min}$ and then was filtered through $\mathrm{SiO}_{2}$. Purification of the 
residue by column chromatography (RP C18, $\left.\mathrm{MeOH} / \mathrm{H}_{2} \mathrm{O}, 9 / 1\right)$ afforded $170 \mathrm{mg}$ (79 \%) of (E)-4(1-heptenyl)acetophenone $((E)-\mathbf{1 1})$ as a yellow oil.

Data for $(E)-\mathbf{1 1}$ :

${ }^{1}$ H NMR: (400 MHz, $\mathrm{CDCl}_{3}$ ) $7.89(\mathrm{~d}, J=8.4,2 \mathrm{H}), 7.41(\mathrm{~d}, J=8.3,2 \mathrm{H}), 6.39$ (m, $2 \mathrm{H}), 2.58(\mathrm{~s}, 3 \mathrm{H}), 2.24(\mathrm{qd}, J=7.4$, 1.6, $2 \mathrm{H}), 1.49$ (qn, $J=7.2,2 \mathrm{H}), 1.33(\mathrm{~m}, 4 \mathrm{H}), 0.91$ (t, $J=7.0,3 \mathrm{H}$ ).

${ }^{13} \underline{\mathrm{C} \mathrm{NMR}}:\left(100.6 \mathrm{MHz}, \mathrm{CDCl}_{3}\right)$

$197.6,142.7,135.4,134.6,128.9,128.7,125.9,33.2,31.4,28.8,26.5,22.5,14.0$.

TLC: $R_{f} 0.39\left(\mathrm{MeOH} / \mathrm{H}_{2} \mathrm{O}, 9 / 1\right)$.

Reaction of 4-Iodoacetophenone with $(E)$-Diisopropyl-(1-heptenyl)silanol $((E)-2)$ in the Presence of Tetra- $n$-butylammonium Fluoride (2 equiv) [DW-IV-95].

Following the General Procedure, $(E)-2(251 \mathrm{mg}, 1.1$ equiv) was dissolved in TBAF solution ( $2 \mathrm{~mL}, 1.0 \mathrm{M}$ in THF, $2.0 \mathrm{mmol})$ and THF $(1 \mathrm{~mL})$ and was stirred for $10 \mathrm{~min}$ at rt. 4Iodoacetophenone $(246 \mathrm{mg}, 1.0 \mathrm{mmol})$ and $\mathrm{Pd}(\mathrm{dba})_{2}(29 \mathrm{mg}, 0.05$ equiv) were added and the mixture was stirred at $\mathrm{rt}$ for $10 \mathrm{~min}$ and then was filtered through $\mathrm{SiO}_{2}$. Purification by column chromatography (RP C18, $\left.\mathrm{MeOH} / \mathrm{H}_{2} \mathrm{O}, 9 / 1\right)$ and Kugelrohr distillation afforded $173 \mathrm{mg}(80 \%)$ of $(E)-\mathbf{1 1}$ as colorless oil.

Data for $(E)-\mathbf{1 1}$ :

${ }^{1} \underline{\mathrm{H} \mathrm{NMR}}:\left(400 \mathrm{MHz}, \mathrm{CDCl}_{3}\right)$

$7.89(\mathrm{~d}, J=8.4,2 \mathrm{H}), 7.41(\mathrm{~d}, J=8.3,2 \mathrm{H}), 6.39(\mathrm{~m}, 2 \mathrm{H}), 2.58(\mathrm{~s}, 3 \mathrm{H}), 2.24(\mathrm{qd}, J=7.4$, $1.6,2 \mathrm{H}), 1.49$ (qn, $J=7.2,2 \mathrm{H}), 1.33(\mathrm{~m}, 4 \mathrm{H}), 0.91$ (t, $J=7.0,3 \mathrm{H})$.

${ }^{13}$ C NMR: (100.6 MHz, CDCl3)

$197.6,142.7,135.4,134.6,128.9,128.7,125.9,33.2,31.4,28.8,26.5,22.5,14.0$.

TLC: $\quad R_{f} 0.39\left(\mathrm{MeOH} / \mathrm{H}_{2} \mathrm{O}, 9 / 1\right)$ 
Reaction of 4-Iodoacetophenone with $(E)$-Dimethyl-fluoro-(1-heptenyl)silane $((E)-4)$ in the Presence of Tetra- $n$-butylammonium fluoride (2 equiv) [DW-IV-94].

Following the General Procedure, $(E)-4(174 \mathrm{mg}, 1.0 \mathrm{mmol})$ was dissolved in TBAF solution ( $2 \mathrm{~mL}, 1.0 \mathrm{M}$ in THF, $2.0 \mathrm{mmol})$ and THF $(2 \mathrm{~mL})$ and was stirred for $10 \mathrm{~min}$ at rt. 4Iodoacetophenone (246 mg, $1.0 \mathrm{mmol})$ and $\mathrm{Pd}(\mathrm{dba})_{2}(29 \mathrm{mg}, 0.05$ equiv) were added and the mixture was stirred at $\mathrm{rt}$ for $10 \mathrm{~min}$ and then was filtered through $\mathrm{SiO}_{2}$. Purification of the residue by column chromatography ( $\left.\mathrm{RP} \mathrm{C} 18, \mathrm{MeOH} / \mathrm{H}_{2} \mathrm{O}, 9 / 1\right)$ afforded $169 \mathrm{mg}(78 \%)$ of $(E)$ 4-(1-heptenyl)acetophenone $((E)-\mathbf{1 1})$ as a yellow oil.

Data for $(E)-\mathbf{1 1}$ :

${ }^{1}$ H NMR: (400 MHz, $\mathrm{CDCl}_{3}$ )

$7.89(\mathrm{~d}, J=8.4,2 \mathrm{H}), 7.41(\mathrm{~d}, J=8.3,2 \mathrm{H}), 6.39$ (m, $2 \mathrm{H}), 2.58(\mathrm{~s}, 3 \mathrm{H}), 2.24(\mathrm{qd}, J=7.4$, 1.6, $2 \mathrm{H}), 1.49$ (qn, $J=7.2,2 \mathrm{H}), 1.33(\mathrm{~m}, 4 \mathrm{H}), 0.91$ (t, $J=7.0,3 \mathrm{H})$.

${ }^{13} \mathrm{C} \mathrm{NMR}:\left(100.6 \mathrm{MHz}, \mathrm{CDCl}_{3}\right)$

197.6, 142.7, 135.4, 134.6, 128.9, 128.7, 125.9, 33.2, 31.4, 28.8, 26.5, 22.5, 14.0.

TLC: $R_{f} 0.39\left(\mathrm{MeOH} / \mathrm{H}_{2} \mathrm{O}, 9 / 1\right)$.

Reaction of 4-Iodoacetophenone with (E,E)-1,1,7,7-Tetramethyl-1,7-di-(1-heptenyl)disiloxane $((E, E)-7)$ in the Presence of Tetra-n-butylammonium Fluoride (2 equiv) [DWIV-97].

Following the General Procedure, $(E, E)-7(163 \mathrm{mg}, 0.5 \mathrm{mmol})$ was dissolved in TBAF solution (2 mL, 1.0 M in THF, $2.0 \mathrm{mmol})$ and THF (1 mL) and was stirred for $10 \mathrm{~min}$ at rt. 4Iodoacetophenone (246 mg, $1.0 \mathrm{mmol})$ and $\mathrm{Pd}(\mathrm{dba})_{2}(29 \mathrm{mg}, 0.05$ equiv) were added and the mixture was stirred at $\mathrm{rt}$ for $10 \mathrm{~min}$ and then was filtered through $\mathrm{SiO}_{2}$. Purification of the residue by column chromatography ( $\left.\mathrm{RP} \mathrm{C} 18, \mathrm{MeOH} / \mathrm{H}_{2} \mathrm{O}, 9 / 1\right)$ afforded $178 \mathrm{mg}(82 \%)$ of $(E)$ 4-(1-heptenyl)acetophenone $((E)-\mathbf{1 1})$ as a yellow oil.

Data for $(E)-\mathbf{1 1}$ :

${ }^{1} \underline{\mathrm{H} \mathrm{NMR}}:\left(400 \mathrm{MHz}, \mathrm{CDCl}_{3}\right)$

$7.89(\mathrm{~d}, J=8.4,2 \mathrm{H}), 7.41(\mathrm{~d}, J=8.3,2 \mathrm{H}), 6.39(\mathrm{~m}, 2 \mathrm{H}), 2.58(\mathrm{~s}, 3 \mathrm{H}), 2.24(\mathrm{qd}, J=7.4$, 1.6, $2 \mathrm{H}), 1.49$ (qn, $J=7.2,2 \mathrm{H}), 1.33(\mathrm{~m}, 4 \mathrm{H}), 0.91$ (t, $J=7.0,3 \mathrm{H})$. 
${ }^{13} \underline{\mathrm{C} \mathrm{NMR}}:\left(100.6 \mathrm{MHz}, \mathrm{CDCl}_{3}\right)$

$197.6,142.7,135.4,134.6,128.9,128.7,125.9,33.2,31.4,28.8,26.5,22.5,14.0$.

TLC: $\operatorname{Rf} 0.39\left(\mathrm{MeOH} / \mathrm{H}_{2} \mathrm{O}, 9 / 1\right)$.

Reaction of 4-Iodoacetophenone with $(E)$-Methyl-(1-heptenyl)siletane $((E)-3)$ in the Presence of Tetra-n-butylammonium Fluoride ( 2 equiv) [JY-III-68].

Following the General Procedure, $(E)-3$ (201 mg, $1.1 \mathrm{mmol}, 1.1$ equiv), a solution of TBAF (3 mL, 1.0 M in THF, 3 equiv), 4-iodoacetophenone (246 mg, $1.0 \mathrm{mmol})$ and $\mathrm{Pd}(\mathrm{dba})_{2}$ (29 $\mathrm{mg}, 0.05 \mathrm{mmol}, 0.05$ equiv) were stirred at $\mathrm{rt}$ for $10 \mathrm{~min}$ and the mixture was filtered through $\mathrm{SiO}_{2}$. Purification of the crude product by column chromatography (RP C18, $\mathrm{MeOH} / \mathrm{H}_{2} \mathrm{O}, 9 / 1$ ) and Kugelrohr distillation afforded $182 \mathrm{mg}(84 \%)$ of $(E)-\mathbf{1 1}$ as colorless oil.

Data for $(E)-\mathbf{1 1}$ :

${ }^{1} \underline{\mathrm{H} \mathrm{NMR}}:\left(400 \mathrm{MHz}, \mathrm{CDCl}_{3}\right)$

$7.88\left(\mathrm{~d}, J=8.6, \mathrm{HC}\left(3^{\prime}\right), 1 \mathrm{H}\right), 7.40\left(\mathrm{~d}, J=8.6, \mathrm{HC}\left(2^{\prime}\right), 1 \mathrm{H}\right), 6.38(\mathrm{~m}, 2 \mathrm{H}, \mathrm{HC}(1,2), 2 \mathrm{H})$, 2.57 (s, $\left.\mathrm{H}_{3} \mathrm{C}\left(6^{\prime}\right), 3 \mathrm{H}\right), 2.23\left(\mathrm{td}, J=7.3,5.4, \mathrm{H}_{2} \mathrm{C}(3), 2 \mathrm{H}\right), 1.49$ (q, $\left.J=5.4, \mathrm{H}_{2} \mathrm{C}(4), 2 \mathrm{H}\right)$, $1.33\left(\mathrm{~m}, \mathrm{H}_{2} \mathrm{C}(5,6), 4 \mathrm{H}\right), 0.90\left(\mathrm{t}, J=6.8, \mathrm{H}_{3} \mathrm{C}(7), 3 \mathrm{H}\right)$

${ }^{13} \underline{\mathrm{C} \mathrm{NMR}}:\left(100.6 \mathrm{MHz}, \mathrm{CDCl}_{3}\right)$

$197.49\left(\mathrm{C}\left(5^{\prime}\right)\right), 142.60\left(\mathrm{C}\left(4^{\prime}\right)\right), 135.29\left(\mathrm{C}\left(1^{\prime}\right)\right), 134.50(\mathrm{C}(2)), 128.83(\mathrm{C}(1)), 128.67$ (C(3')), $125.82\left(\mathrm{C}\left(2^{\prime}\right)\right), 33.09(\mathrm{C}(3)), 31.38(\mathrm{C}(5)), 28.76(\mathrm{C}(4)), 26.46\left(\mathrm{C}\left(6^{\prime}\right)\right), 22.48$ $(\mathrm{C}(6)), 13.98(\mathrm{C}(7))$

TLC: $R_{f} 0.39\left(\mathrm{RP} \mathrm{C} 18, \mathrm{MeOH} / \mathrm{H}_{2} \mathrm{O}, 9 / 1\right)$ 


\section{NMR-Experiments of Organosilicon Precursors with TBAF (Figure 4).}

\section{Reaction of $(E)$-Dimethyl-(1-heptenyl)silanol ( $(E)-1)$ with TBAF [DW-V-17]}

Silanol $(E)-1(8.6 \mathrm{mg}, 0.05 \mathrm{mmol})$ was dissolved in THF- $d_{8}(0.5 \mathrm{~mL})$ in a dry NMR-tube under $\mathrm{N}_{2}$. TBAF (16 mg, $0.05 \mathrm{mmol}$ ) was added and the NMR spectra were recorded. The disiloxane $(E, E)-7$ and the unknown $\mathbf{X}$ could be detected in a 1/1 ratio.

Data for $(E, E)-7$ :

${ }^{1}$ H NMR: $\left(500 \mathrm{MHz}, \mathrm{THF}-d_{8}\right)$

$6.13(\mathrm{dt}, J=18.7,6.2 \mathrm{~Hz}, \mathrm{HC}(2), 2 \mathrm{H}) ; 5.62(\mathrm{dt}, J=18.7,1.5 \mathrm{~Hz}, \mathrm{HC}(1), 2 \mathrm{H}) ; 0.10$ (s, $\left.\mathrm{HC}\left(1^{\prime}\right), 12 \mathrm{H}\right)$.

${ }^{29} \underline{\mathrm{Si} N M R}:\left(99.3 \mathrm{MHz}, \mathrm{THF}-d_{8}\right)$

-3.93 .

Data for $\mathbf{X}$ :

$\left.{ }^{1} \underline{\text { H NMR: }} 500 \mathrm{MHz}, \mathrm{THF}-d_{8}\right)$

$6.08(\mathrm{dt}, J=18.5,6.5 \mathrm{~Hz}, \mathrm{HC}(2), 2 \mathrm{H}) ; 5.65$ (dt, $J=18.5,1.0 \mathrm{~Hz}, \mathrm{HC}(1), 2 \mathrm{H}) ; 0.08$ (s, $\left.\mathrm{HC}\left(1^{\prime}\right), 6 \mathrm{H}\right)$.

${ }^{29} \underline{\text { Si NMR: }}\left(99.3 \mathrm{MHz}, \mathrm{THF}-d_{8}\right)$

-7.68 .

\section{Reaction of $(E, E)-1,1,7,7-T e t r a m e t h y l-1,7-d i-(1-h e p t e n y l) d i s i l o x a n e ~((E, E)-7)$ with TBAF [DW-V-21]}

Disiloxane $(E, E)-7(8.2 \mathrm{mg}, 0.025 \mathrm{mmol})$ was dissolved in THF- $d_{8}(0.5 \mathrm{~mL})$ in a dry NMR-tube under $\mathrm{N}_{2}$. TBAF (16 mg, $0.05 \mathrm{mmol}$ ) was added and the NMR spectra were recorded. The disiloxane $(E, E)-\mathbf{7}$ and the unknown $\mathbf{X}$ could be detected in a 1/1 ratio.

Data for $(E, E)-7$ :

${ }^{1}$ H NMR: $\left(500 \mathrm{MHz}, \mathrm{THF}-d_{8}\right)$

$6.13(\mathrm{dt}, J=18.7,6.2 \mathrm{~Hz}, \mathrm{HC}(2), 2 \mathrm{H}) ; 5.62$ (dt, $J=18.7,1.5 \mathrm{~Hz}, \mathrm{HC}(1), 2 \mathrm{H}) ; 0.10$ (s, $\left.\mathrm{HC}\left(1^{\prime}\right), 12 \mathrm{H}\right)$.

${ }^{29} \underline{\text { Si NMR: }}\left(99.3 \mathrm{MHz}, \mathrm{THF}-d_{8}\right)$

-3.93 . 
Data for $\mathbf{X}$ :

${ }^{1}$ H NMR: $\left.500 \mathrm{MHz}, \mathrm{THF}-d_{8}\right)$

$6.08(\mathrm{dt}, J=18.5,6.5 \mathrm{~Hz}, \mathrm{HC}(2), 2 \mathrm{H}) ; 5.65$ (dt, $J=18.5,1.0 \mathrm{~Hz}, \mathrm{HC}(1), 2 \mathrm{H}) ; 0.08$ (s, $\left.\mathrm{HC}\left(1^{\prime}\right), 6 \mathrm{H}\right)$.

${ }^{29} \underline{\text { Si NMR: }}\left(99.3 \mathrm{MHz}, \mathrm{THF}-d_{8}\right)$

-7.68 .

\section{Reaction of $(E)$-Dimethylfluoro-(1-heptenyl)silane ((E)-4) with TBAF [DW-V-18]}

Fluorosilane $(E)-4(8.8 \mathrm{mg}, 0.05 \mathrm{mmol})$ was dissolved in THF- $d_{8}(0.5 \mathrm{~mL})$ in a dry NMR-tube under $\mathrm{N}_{2}$. TBAF (16 mg, $0.05 \mathrm{mmol}$ ) was added and the NMR spectra were recorded. The disiloxane $(E, E)-\mathbf{7}$ and the unknown $\mathbf{X}$ could be detected in a 1/1 ratio.

Data for $(E, E)-7$ :

${ }^{1} \underline{\text { H NMR: }}\left(500 \mathrm{MHz}, \mathrm{THF}-d_{8}\right)$

$6.13(\mathrm{dt}, J=18.7,6.2 \mathrm{~Hz}, \mathrm{HC}(2), 2 \mathrm{H}) ; 5.62$ (dt, $J=18.7,1.5 \mathrm{~Hz}, \mathrm{HC}(1), 2 \mathrm{H}) ; 0.10$ (s, $\left.\mathrm{HC}\left(1^{\prime}\right), 12 \mathrm{H}\right)$.

${ }^{29} \underline{\text { Si NMR: }}\left(99.3 \mathrm{MHz}, \mathrm{THF}-d_{8}\right)$

-3.92 .

\section{Data for $\mathbf{X}^{\prime}$}

${ }^{1}$ H NMR: $\left.500 \mathrm{MHz}, \mathrm{THF}-d_{8}\right)$

$6.08(\mathrm{dt}, J=18.5,6.5 \mathrm{~Hz}, \mathrm{HC}(2), 2 \mathrm{H}) ; 5.65(\mathrm{dt}, J=18.5,1.0 \mathrm{~Hz}, \mathrm{HC}(1), 2 \mathrm{H}) ; 0.08$ (s, $\left.\mathrm{HC}\left(1^{\prime}\right), 6 \mathrm{H}\right)$.

${ }^{29} \underline{\text { Si NMR: }}\left(99.3 \mathrm{MHz}, \mathrm{THF}-d_{8}\right)$

-4.20 .

\section{Reaction of $(E)$-Diisopropyl-(1-heptenyl)silanol ((E)-2) with TBAF [DW-V-17]}

Silanol $(E)-2(11.4 \mathrm{mg}, 0.05 \mathrm{mmol})$ was dissolved in THF- $d_{8}(0.5 \mathrm{~mL})$ in a dry NMRtube under $\mathrm{N}_{2}$. TBAF (16 mg, $0.05 \mathrm{mmol}$ ) was added and the NMR spectra were recorded. The disiloxane $(E, E)-8$ and the unknown $\mathbf{Y}$ could be detected in a 1/1 ratio. 
Data for $(E, E)-8$ :

$\left.{ }^{1} \underline{\mathrm{H} \text { NMR}}: 500 \mathrm{MHz}, \mathrm{THF}-d_{8}\right)$

$6.24(\mathrm{dt}, J=18.8,6.5 \mathrm{~Hz}, \mathrm{HC}(2), 1 \mathrm{H} ; 5.57$ (dt, $J=18.8,1.5 \mathrm{~Hz}, \mathrm{HC}(1), 1 \mathrm{H})$.

${ }^{29} \underline{\mathrm{Si} N M R}:\left(99.3 \mathrm{MHz}, \mathrm{THF}-d_{8}\right)$

-3.55 .

Data for $\mathbf{Y}\left({ }^{1} \underline{\mathrm{H}} \mathrm{NMR}\right.$ data is coincident with $(E)-2,{ }^{29} \mathrm{Si}$ NMR is not):

$\left.{ }^{1} \underline{\mathrm{H} \text { NMR: }} 500 \mathrm{MHz}, \mathrm{THF}-d_{8}\right)$

$6.20(\mathrm{dt}, J=18.6,6.3 \mathrm{~Hz}, \mathrm{HC}(2), 1 \mathrm{H} ; 5.53$ (dt, $J=18.6,1.5 \mathrm{~Hz}, \mathrm{HC}(1), 1 \mathrm{H})$.

${ }^{29} \underline{\mathrm{Si} N M R}:\left(99.3 \mathrm{MHz}, \mathrm{THF}-d_{8}\right)$

-4.63 .

\section{Reaction of $(E)$-Methyl-(1-heptenyl)siletane ((E)-3) with TBAF [DW-V-14]}

Silacyclobutane $(E)-3(9.1 \mathrm{mg}, 0.05 \mathrm{mmol})$ was dissolved in THF- $d_{8}(0.5 \mathrm{~mL})$ in a dry NMR-tube under $\mathrm{N}_{2}$. TBAF (16 mg, $0.05 \mathrm{mmol}$ ) was added and the NMR spectra were recorded. Disiloxane (E,E)-6 and the unknown $\mathbf{Z}$ could be detected in a 1/1 ratio.

Data for $(E, E)-6$ :

${ }^{1}$ H NMR: (400 MHz, THF- $\left.d_{8}\right)$

$6.09(\mathrm{dt}, J=18.6,6.4 \mathrm{~Hz}, \mathrm{HC}(2), 2 \mathrm{H}) ; 5.55$ (d, $J=18.6 \mathrm{~Hz}, \mathrm{HC}(1), 2 \mathrm{H}) ; 0.50$ (t, $J=5.9$ $\left.\mathrm{Hz}, \mathrm{HC}\left(1^{\prime \prime}\right), 4 \mathrm{H}\right) ;-0.02$ (s, HC(1'), $\left.6 \mathrm{H}\right)$.

${ }^{29} \underline{\text { Si NMR: }}\left(99.3 \mathrm{MHz}, \mathrm{THF}-d_{8}\right)$ -4.24 .

Data for $\mathbf{Z}$ :

${ }^{1} \underline{\mathrm{H} N M R}:\left(400 \mathrm{MHz}, \mathrm{THF}-d_{8}\right)$

$0.58(\mathrm{t}, J=8.3 \mathrm{~Hz}, 2 \mathrm{H}) ; 0.09(\mathrm{~s}, 3 \mathrm{H})$.

${ }^{29} \underline{\text { Si NMR: }}\left(99.3 \mathrm{MHz}, \mathrm{THF}-d_{8}\right)$

-8.45 . 


\section{NMR-Titration Experiments of Organosilicon Precursors with TBAF (Figure 5).}

To an NMR tube containing $1 \mathrm{~mL}$ of THF- $d_{8}$ and either $(E)-\mathbf{1}(17.2 \mathrm{mg}, 0.1 \mathrm{mmol})$ or (E)-2 $(22.8 \mathrm{mg}, 0.1 \mathrm{mmol})$ or $(E)-\mathbf{2}(22.8 \mathrm{mg}, 0.1 \mathrm{mmol})(E)-3 \mathrm{or}(9.1 \mathrm{mg}, 0.05 \mathrm{mmol})$ was added the appropriate amount of TBAF (1.6 mg - 5\%, $31.6 \mathrm{mg}$ - 1 equiv, or $63.1 \mathrm{mg}$ - 2 equiv) The resultant solution was analyzed by ${ }^{1} \mathrm{H}$ NMR spectroscopy 30 min later. The ratio of $\mathbf{X}, \mathbf{Y}$, or $\mathbf{Z}$ / disiloxane was determined by integration the proton signals of the silicon-bonded methyl groups for $(E, E)-\mathbf{6} / \mathbf{Z},(E, E)-7 / \mathbf{X}$ or by integration of the olefinic proton $\mathrm{HC}(1)$ for $(E)-\mathbf{8} / \mathbf{Y})$ 


\section{Determination of the Response Factor of $(E)-16$ and Biphenyl (A).}

Stock solutions of (E)-16 (69.4 mg in $10.0 \mathrm{~mL}$ dry THF) and biphenyl (69.5 mg in $10.0 \mathrm{~mL}$ dry THF) were prepared in volumetric flasks. The amounts indicated in column 1 for biphenyl and column 4 for 7 were withdrawn and mixed in a $1.0 \mathrm{~mL}$ volumetric flask which was filled to the mark. These samples were injected into the GC $(1 \mu \mathrm{L})$ three times to give the average areas indicated in columns 3 (A) and 6 (16). Columns 2 and 5 indicate the actual amounts of biphenyl and $(E)-\mathbf{1 6}$ injected. The response factor for every sample was calculated by :

Response factor $\quad=\quad(\mathrm{mg} \mathrm{16} *$ area $\mathbf{A}) /(\operatorname{area} 16 * \operatorname{mg~A})$

\begin{tabular}{c|c|c|c|c|c|c}
\hline$\mu \mathrm{L}$ sol. A & mg A & area A & $\mu \mathrm{L}$ sol.16 & $\mathrm{mg} \mathbf{1 6}$ & area 16 & response factor \\
\hline 100 & 0.695 & 6660 & 50 & 0.347 & 2064 & 1.61 \\
100 & 0.695 & 6868 & 50 & 0.347 & 2073 & 1.65 \\
100 & 0.695 & 6673 & 100 & 0.694 & 3955 & 1.68 \\
100 & 0.695 & 6787 & 100 & 0.694 & 4048 & 1.67 \\
100 & 0.695 & 6929 & 150 & 1.041 & 6121 & 1.70 \\
100 & 0.695 & 6973 & 150 & 1.041 & 6200 & 1.68 \\
100 & 0.695 & 6920 & 200 & 1.388 & 7931 & 1.74 \\
100 & 0.695 & 6839 & 200 & 1.388 & 7878 & 1.73 \\
50 & 0.3475 & 3934 & 150 & 1.041 & 6494 & 1.81 \\
50 & 0.3475 & 4180 & 150 & 1.041 & 6739 & 1.86 \\
50 & 0.3475 & 4198 & 200 & 1.388 & 8889 & 1.89 \\
50 & 0.3475 & 4136 & 200 & 1.388 & 8733 & 1.89 \\
200 & 1.39 & 13501 & 150 & 1.041 & 6180 & 1.64 \\
200 & 1.39 & 14997 & 150 & 1.041 & 6878 & 1.63 \\
200 & 1.39 & 14177 & 250 & 1.735 & 10991 & 1.61 \\
200 & 1.39 & 13800 & 250 & 1.735 & 10646 & 1.62 \\
200 & 1.39 & 13006 & 150 & 1.041 & 6079 & 1.60 \\
200 & 1.39 & 12954 & 150 & 1.041 & 6063 & 1.60 \\
\hline & & & & & average & 1.70 \\
\hline
\end{tabular}




\section{Evaluation of Procedure to Quench the Cross-Coupling Reaction with 2- Dimethylaminoethanethiol Hydrochloride}

In a flame dried, 2-neck, round-bottomed flask, $(E)-1$ (43 mg, $0.25 \mathrm{mmol})$ and biphenyl (12.3 mg) were dissolved in dry THF $(2.70 \mathrm{~mL})$ under argon. A solution of TBAF (1.25 mL, 1.0 $\mathrm{M}$ in THF, $1.25 \mathrm{mmol})$ was added, followed by 2-iodothiophene $(28 \mathrm{~mL}, 0.25 \mathrm{mmol})$ and a suspension of bis(dibenzylideneacetone)palladium $(7.2 \mathrm{mg}, 0.013 \mathrm{mmol})$ in dry THF (1.0 mL). The reaction was then stirred at $\mathrm{rt}$ for $30 \mathrm{~min}$. At this time $200 \mu \mathrm{L}$ of the reaction mixture were withdrawn and filtered through a plug of silica gel, which was then washed with diethyl ether. At the same time, a solution of 2-dimethylaminoethanethiol hydrochloride $(2.0 \mathrm{~mL}, 20 \%$, aqueous) was added to the vigorously stirred reaction mixture. Aliquots of the reaction $(300 \mathrm{~mL})$ were withdrawn after 30,60 and 120 min and were filtered through a plug of silica gel. The four samples were analyzed by GC. The same value for conversion to product was obtained, indicating the 2-dimethylaminoethanethiol hydrochloride solution effectively quenched the reaction.

Retention times (HP 5, 200 C, 15 psi):

$t_{R}(\mathbf{A}): 7.00 \mathrm{~min}$.

$t_{R}$ (16): $7.41 \mathrm{~min}$.

\begin{tabular}{|c|c|c|c|}
\hline time (min) & area A & area 16 & conversion (\%) \\
\hline 0 & 10209 & 5366 & 24 \\
\hline 30 & 9101 & 4778 & 24 \\
\hline 60 & 9032 & 4709 & 24 \\
\hline 120 & 8781 & 4584 & 24 \\
\hline
\end{tabular}




\section{General Procedure for the Kinetic Measurements}

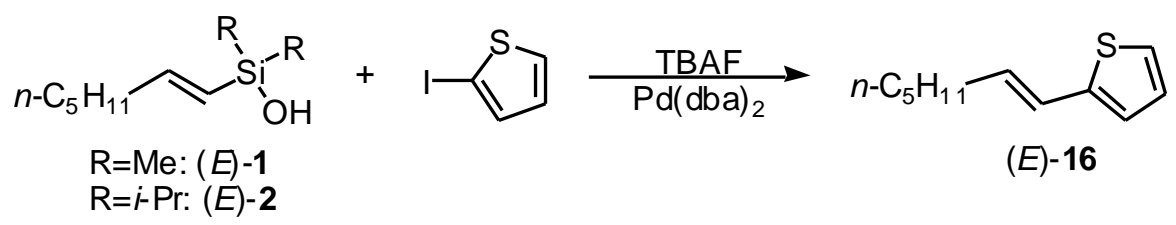

(E)-1 or (E)-2 and biphenyl were dissolved in TBAF solution (1.0 M in THF) in a flamedried 2-neck, round-bottomed flask under argon. Dry THF was added to adjust the total volume to either 2.0 or $4.0 \mathrm{~mL}$. The flask was immersed into a water-bath for $30 \mathrm{~min}$. and the reactionmixture's temperature was adjusted to $23{ }^{\circ} \mathrm{C} \pm 1{ }^{\circ} \mathrm{C}$. The 2-iodothiophene was then added, and the reaction was stirred for $1 \mathrm{~min}$. A suspension of bis(dibenzylideneacetone)palladium in 1.0 $\mathrm{mL}$ of dry THF was then added. The purple, turbid reaction mixture turns clear within 30 seconds. Aliquots $(200 \mu \mathrm{L})$ were removed and quenched after 1, 2, 3, 4, 5, 7, 10, 15, 20, 30, 40, $50,60,75,90,120 \mathrm{~min}$. The quench was performed as follows: the withdrawn aliquot of the reaction mixture was injected into an aqueous $20 \%$ solution of 2-dimethylaminoethanethiol hydrochloride. This colorless to yellow clear solution was filtered through a plug of silica gel. The plug was washed with diethyl ether to adjust the total sample volume to $\sim 2 \mathrm{~mL}$. This sample was subjected to GC analysis. All injections were made in triplicate and the progress of the coupling was monitored as a molar conversion.

Retention times (HP 5, 200 $\mathrm{C}, 15 \mathrm{psi})$ :

$t_{R}(\mathbf{A}): 7.00 \mathrm{~min}$.

$t_{R}(\mathbf{1 6}): 7.41 \mathrm{~min}$. 


\section{Order in 2-Iodothiophene, 0.5 equiv.}

(E)-1

2-Iodothiophene

Tetra- $n$-butylammonium fluoride

Bis(dibenzylideneacetone)palladium $2.5 \mathrm{mM}$ (0.05 equiv)
$50 \mathrm{mM}$ (1.0 equiv)

$25 \mathrm{mM}(0.5$ equiv $)$

$188 \mathrm{mM}$ (3.75 equiv)

Following the General Procedure, silanol (E)-1 (43 mg, $0.25 \mathrm{mmol})$ and biphenyl (12.6 $\mathrm{mg}$ ) were dissolved in TBAF solution $(940 \mu \mathrm{L}, 1.0 \mathrm{M}$ in THF, $0.94 \mathrm{mmol})$ in a flame dried 2neck round-bottomed flask, equipped with a stir bar and a septum under argon. Dry THF (3.0 $\mathrm{mL}$ ) was added and the clear, slightly-yellow solution was immersed into a water-bath at $23{ }^{\circ} \mathrm{C} \pm$ $1{ }^{\circ} \mathrm{C}$ for $30 \mathrm{~min}$. The 2 -iodothiophene $(14 \mu \mathrm{L}, 0.125 \mathrm{mmol})$ was then added and the reaction was stirred for $1 \mathrm{~min}$. A suspension of $\mathrm{Pd}(\mathrm{dba})_{2}(7.2 \mathrm{mg}, 0.0125 \mathrm{mmol})$ in dry THF $(1.0 \mathrm{~mL})$ was added. The initially purple, turbid reaction mixture turns into a clear solution within 30 seconds. Aliquots $(\sim 200 \mu \mathrm{L})$ were withdrawn after 1, 2, 3, 4, 5, 7, 10, 15, 20, 30, 40, 50, 60, 75, 90, and $120 \mathrm{~min}$. To quench, these samples were added to $100 \mu \mathrm{L}$ of a $20 \%$ aqueous solution of 2dimethylaminoethanethiol hydrochloride. The clear colorless to slightly-yellow solution was then filtered through a plug of silica gel, and the silica gel was washed with diethyl ether to give a total sample volume of $\sim 2 \mathrm{~mL}$. This sample was injected into the GC three times.

initial rate $=0.0131$

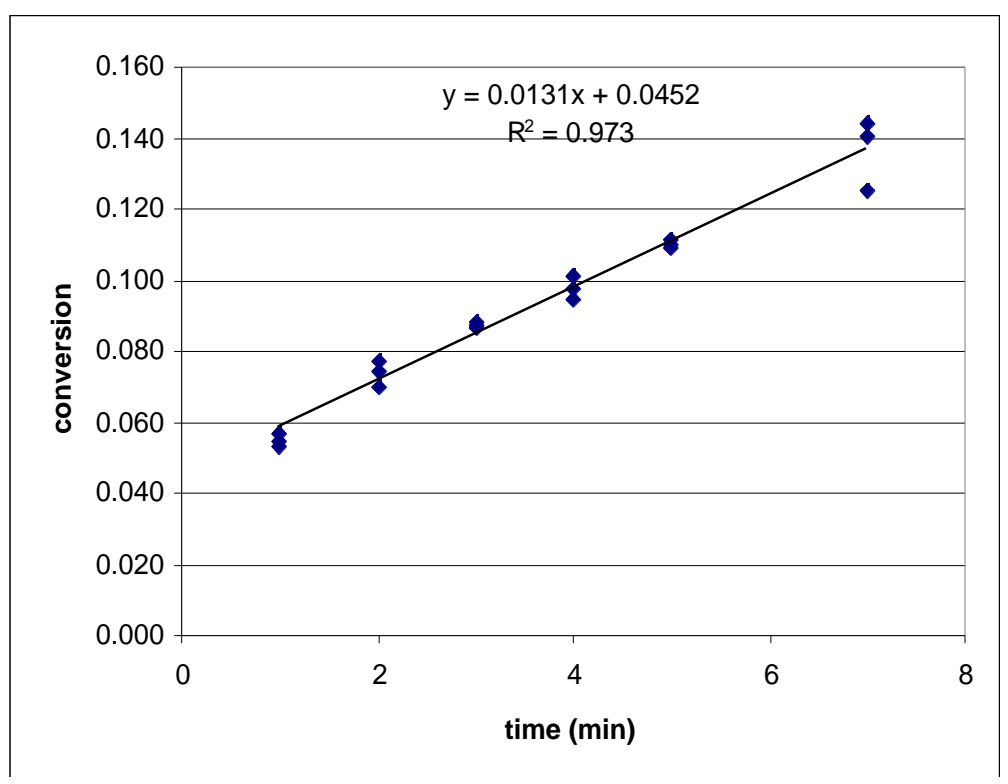




\begin{tabular}{|c|c|c|c|}
\hline time & area $\mathrm{A}$ & area 16 & conversion \\
\hline 1 & 3982 & 478 & 0.057 \\
\hline 1 & 5018 & 562 & 0.053 \\
\hline 1 & 4115 & 471 & 0.054 \\
\hline 2 & 3433 & 534 & 0.074 \\
\hline 2 & 3852 & 624 & 0.077 \\
\hline 2 & 3553 & 522 & 0.070 \\
\hline 3 & 3552 & 654 & 0.087 \\
\hline 3 & 3894 & 711 & 0.087 \\
\hline 3 & 3285 & 609 & 0.088 \\
\hline 4 & 3527 & 702 & 0.095 \\
\hline 4 & 4018 & 821 & 0.097 \\
\hline 4 & 3403 & 725 & 0.101 \\
\hline 5 & 3651 & 837 & 0.109 \\
\hline 5 & 3416 & 799 & 0.111 \\
\hline 5 & 3419 & 788 & 0.110 \\
\hline 7 & 2858 & 752 & 0.125 \\
\hline 7 & 2818 & 832 & 0.140 \\
\hline 7 & 2378 & 719 & 0.144 \\
\hline 10 & 3550 & 1170 & 0.157 \\
\hline 10 & 4285 & 1461 & 0.162 \\
\hline 10 & 3435 & 1196 & 0.165 \\
\hline 15 & 4434 & 1928 & 0.207 \\
\hline 15 & 4795 & 2078 & 0.206 \\
\hline 15 & 3989 & 1796 & 0.214 \\
\hline 20 & 6611 & 3476 & 0.250 \\
\hline 20 & 7034 & 3692 & 0.249 \\
\hline 20 & 6118 & 3183 & 0.247 \\
\hline 30 & 6360 & 4229 & 0.316 \\
\hline 30 & 6560 & 4361 & 0.316 \\
\hline 30 & 6222 & 4108 & 0.314 \\
\hline 40 & 5495 & 4388 & 0.379 \\
\hline 40 & 5985 & 4792 & 0.380 \\
\hline 40 & 5071 & 4053 & 0.380 \\
\hline 50 & 4719 & 4309 & 0.434 \\
\hline 50 & 5647 & 5097 & 0.429 \\
\hline 50 & 4362 & 3903 & 0.425 \\
\hline 60 & 5749 & 5781 & 0.478 \\
\hline 60 & 6484 & 6568 & 0.481 \\
\hline 60 & 5553 & 5660 & 0.484 \\
\hline 75 & 5651 & 6234 & 0.524 \\
\hline 75 & 6446 & 7048 & 0.520 \\
\hline 75 & 5387 & 6019 & 0.531 \\
\hline 90 & 5074 & 5995 & 0.561 \\
\hline 90 & 5868 & 6913 & 0.560 \\
\hline 90 & 5739 & 6802 & 0.563 \\
\hline 120 & 4549 & 5533 & 0.578 \\
\hline 120 & 5626 & 6758 & 0.571 \\
\hline
\end{tabular}




\section{Order in 2-Iodothiophene, 0.5 equiv.}

(E)-1

2-Iodothiophene

Tetra- $n$-butylammonium fluoride

Bis(dibenzylideneacetone)palladium $2.5 \mathrm{mM}$ (0.05 equiv)
$50 \mathrm{mM}$ (1.0 equiv)

$25 \mathrm{mM}(0.5$ equiv $)$

$188 \mathrm{mM}$ (3.75 equiv)

Following the General Procedure, silanol (E)-1 (43 mg, $0.25 \mathrm{mmol})$ and biphenyl (10.1 $\mathrm{mg}$ ) were dissolved in TBAF solution $(940 \mu \mathrm{L}, 1.0 \mathrm{M}$ in THF, $0.94 \mathrm{mmol})$ in a flame dried 2neck round-bottomed flask, equipped with a stir bar and a septum under argon. Dry THF (3.0 $\mathrm{mL}$ ) was added and the clear, slightly-yellow solution was immersed into a water-bath at $23{ }^{\circ} \mathrm{C} \pm$ $1{ }^{\circ} \mathrm{C}$ for $30 \mathrm{~min}$. The 2 -iodothiophene $(14 \mu \mathrm{L}, 0.125 \mathrm{mmol})$ was then added and the reaction was stirred for $1 \mathrm{~min}$. A suspension of $\operatorname{Pd}(\mathrm{dba})_{2}(7.2 \mathrm{mg}, 0.0125 \mathrm{mmol})$ in dry THF $(1.0 \mathrm{~mL})$ was added. The initially purple, turbid reaction mixture turns into a clear solution within 30 seconds. Aliquots $(\sim 200 \mu \mathrm{L})$ were withdrawn after 1, 2, 3, 4, 5, 7, 10, 15, 20, 30, 40, 50, 60, 75, 90, and $120 \mathrm{~min}$. To quench, these samples were added to $100 \mu \mathrm{L}$ of a $20 \%$ aqueous solution of 2dimethylaminoethanethiol hydrochloride. The clear colorless to slightly-yellow solution was then filtered through a plug of silica gel, and the silica gel was washed with diethyl ether to give a total sample volume of $\sim 2 \mathrm{~mL}$. This sample was injected into the GC three times.

initial rate $=0.0105$

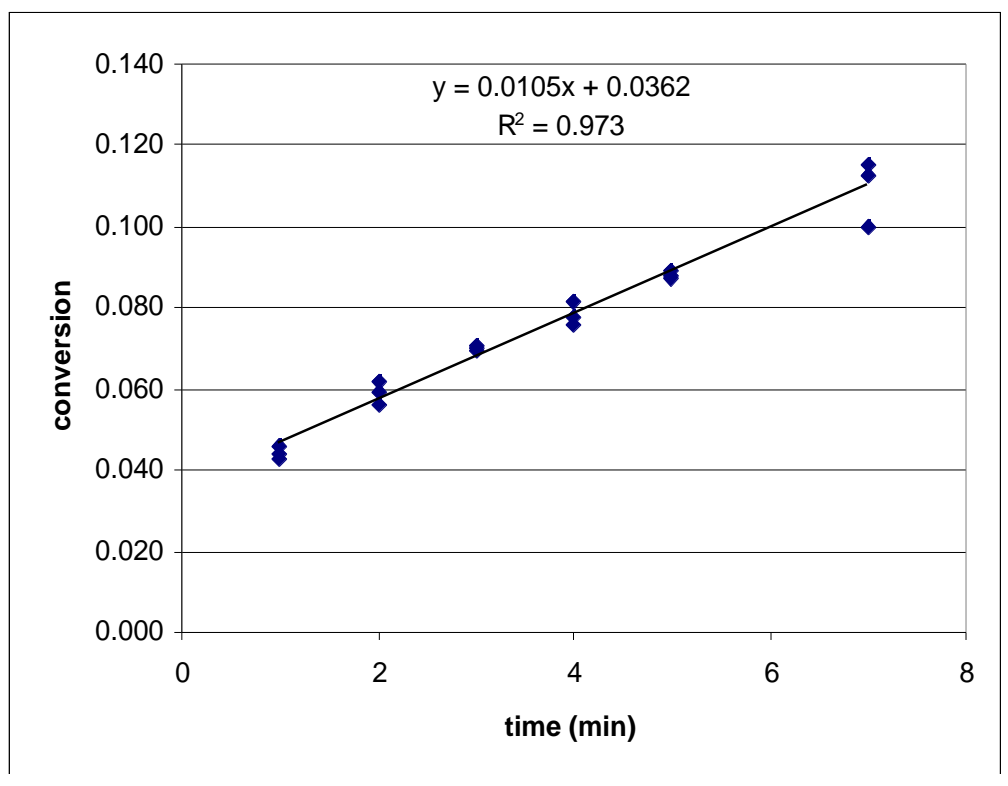




\begin{tabular}{|c|c|c|c|}
\hline time & $\operatorname{area} \mathrm{A}$ & area 16 & conversion \\
\hline 1 & 3982 & 478 & 0.046 \\
\hline 1 & 5018 & 562 & 0.043 \\
\hline 1 & 4115 & 471 & 0.044 \\
\hline 2 & 3433 & 534 & 0.059 \\
\hline 2 & 3852 & 624 & 0.062 \\
\hline 2 & 3553 & 522 & 0.056 \\
\hline 3 & 3552 & 654 & 0.070 \\
\hline 3 & 3894 & 711 & 0.070 \\
\hline 3 & 3285 & 609 & 0.071 \\
\hline 4 & 3527 & 702 & 0.076 \\
\hline 4 & 4018 & 821 & 0.078 \\
\hline 4 & 3403 & 725 & 0.081 \\
\hline 5 & 3651 & 837 & 0.087 \\
\hline 5 & 3416 & 799 & 0.089 \\
\hline 5 & 3419 & 788 & 0.088 \\
\hline 7 & 2858 & 752 & 0.100 \\
\hline 7 & 2818 & 832 & 0.112 \\
\hline 7 & 2378 & 719 & 0.115 \\
\hline 10 & 3550 & 1170 & 0.126 \\
\hline 10 & 4285 & 1461 & 0.130 \\
\hline 10 & 3435 & 1196 & 0.133 \\
\hline 15 & 4434 & 1928 & 0.166 \\
\hline 15 & 4795 & 2078 & 0.165 \\
\hline 15 & 3989 & 1796 & 0.171 \\
\hline 20 & 6611 & 3476 & 0.200 \\
\hline 20 & 7034 & 3692 & 0.200 \\
\hline 20 & 6118 & 3183 & 0.198 \\
\hline 30 & 6360 & 4229 & 0.253 \\
\hline 30 & 6560 & 4361 & 0.253 \\
\hline 30 & 6222 & 4108 & 0.251 \\
\hline 40 & 5495 & 4388 & 0.304 \\
\hline 40 & 5985 & 4792 & 0.305 \\
\hline 40 & 5071 & 4053 & 0.304 \\
\hline 50 & 4719 & 4309 & 0.348 \\
\hline 50 & 5647 & 5097 & 0.344 \\
\hline 50 & 4362 & 3903 & 0.341 \\
\hline 60 & 5749 & 5781 & 0.383 \\
\hline 60 & 6484 & 6568 & 0.386 \\
\hline 60 & 5553 & 5660 & 0.388 \\
\hline 75 & 5651 & 6234 & 0.420 \\
\hline 75 & 6446 & 7048 & 0.416 \\
\hline 75 & 5387 & 6019 & 0.426 \\
\hline 90 & 5074 & 5995 & 0.450 \\
\hline 90 & 5868 & 6913 & 0.449 \\
\hline 90 & 5739 & 6802 & 0.451 \\
\hline 120 & 4549 & 5533 & 0.463 \\
\hline 120 & 5626 & 6758 & 0.458 \\
\hline
\end{tabular}




\section{Order in 2-Iodothiophene, 0.5 equiv.}

(E)-1

2-Iodothiophene

Tetra- $n$-butylammonium fluoride

Bis(dibenzylideneacetone)palladium $2.5 \mathrm{mM}$ (0.05 equiv)
$50 \mathrm{mM}$ (1.0 equiv)

$25 \mathrm{mM}(0.5$ equiv $)$

$188 \mathrm{mM}$ (3.75 equiv)

Following the General Procedure, silanol (E)-1 (43 mg, $0.25 \mathrm{mmol})$ and biphenyl (8.8 $\mathrm{mg}$ ) were dissolved in TBAF solution $(940 \mu \mathrm{L}, 1.0 \mathrm{M}$ in THF, $0.94 \mathrm{mmol})$ in a flame dried 2neck round-bottomed flask, equipped with a stir bar and a septum under argon. Dry THF (3.0 $\mathrm{mL}$ ) was added and the clear, slightly-yellow solution was immersed into a water-bath at $23{ }^{\circ} \mathrm{C} \pm$ $1{ }^{\circ} \mathrm{C}$ for $30 \mathrm{~min}$. The 2 -iodothiophene $(14 \mu \mathrm{L}, 0.125 \mathrm{mmol})$ was then added and the reaction was stirred for $1 \mathrm{~min}$. A suspension of $\operatorname{Pd}(\mathrm{dba})_{2}(7.2 \mathrm{mg}, 0.0125 \mathrm{mmol})$ in dry THF $(1.0 \mathrm{~mL})$ was added. The initially purple, turbid reaction mixture turns into a clear solution within 30 seconds. Aliquots $(\sim 200 \mu \mathrm{L})$ were withdrawn after 1, 2, 3, 4, 5, 7, 10, 15, 20, 30, 40, 50, 60, 75, 90, and $120 \mathrm{~min}$. To quench, these samples were added to $100 \mu \mathrm{L}$ of a $20 \%$ aqueous solution of 2dimethylaminoethanethiol hydrochloride. The clear colorless to slightly-yellow solution was then filtered through a plug of silica gel, and the silica gel was washed with diethyl ether to give a total sample volume of $\sim 2 \mathrm{~mL}$. This sample was injected into the GC three times.

initial rate $=0.0093$

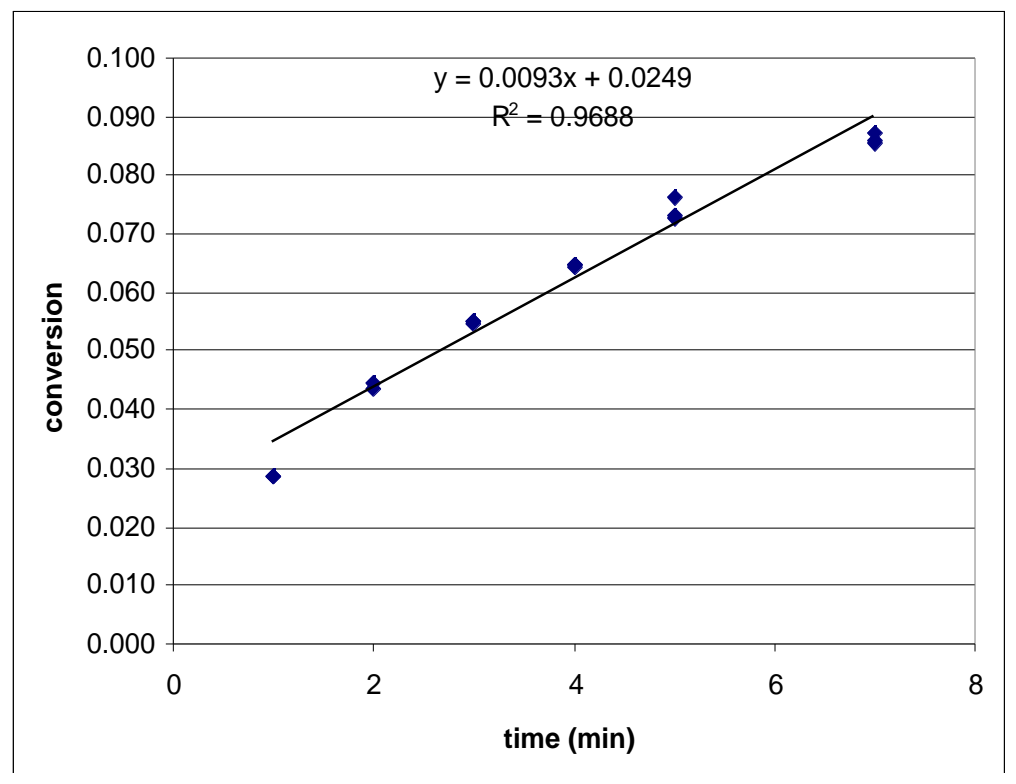




\begin{tabular}{|c|c|c|c|}
\hline time & area standard & area 16 & conversion \\
\hline 1 & 35481 & 3071 & 0.029 \\
\hline 1 & 45355 & 3902 & 0.029 \\
\hline 2 & 11510 & 1547 & 0.045 \\
\hline 2 & 38897 & 5110 & 0.044 \\
\hline 3 & 11239 & 1858 & 0.055 \\
\hline 3 & 30456 & 5035 & 0.055 \\
\hline 3 & 38764 & 6405 & 0.055 \\
\hline 4 & 9892 & 1925 & 0.065 \\
\hline 4 & 29615 & 5765 & 0.065 \\
\hline 4 & 31324 & 6050 & 0.064 \\
\hline 5 & 10275 & 2359 & 0.076 \\
\hline 5 & 28801 & 6318 & 0.073 \\
\hline 5 & 37153 & 8205 & 0.073 \\
\hline 7 & 13313 & 3499 & 0.087 \\
\hline 7 & 35403 & 9124 & 0.086 \\
\hline 7 & 53243 & 13812 & 0.086 \\
\hline 10 & 5700 & 1834 & 0.107 \\
\hline 10 & 16838 & 5450 & 0.107 \\
\hline 10 & 19280 & 6188 & 0.107 \\
\hline 15 & 5137 & 2130 & 0.138 \\
\hline 15 & 15894 & 6521 & 0.136 \\
\hline 15 & 16856 & 6912 & 0.136 \\
\hline 20 & 5496 & 2733 & 0.165 \\
\hline 20 & 16226 & 8038 & 0.164 \\
\hline 30 & 6268 & 4234 & 0.224 \\
\hline 30 & 13196 & 8901 & 0.224 \\
\hline 30 & 21911 & 14667 & 0.222 \\
\hline 40 & 6154 & 5223 & 0.282 \\
\hline 40 & 18254 & 15004 & 0.273 \\
\hline 40 & 23067 & 19500 & 0.281 \\
\hline 50 & 5702 & 5806 & 0.338 \\
\hline 50 & 17824 & 17895 & 0.333 \\
\hline 50 & 24695 & 24579 & 0.330 \\
\hline 60 & 11954 & 14304 & 0.397 \\
\hline 60 & 38210 & 44358 & 0.385 \\
\hline 75 & 4804 & 6581 & 0.455 \\
\hline 75 & 13749 & 18597 & 0.449 \\
\hline 75 & 14316 & 19160 & 0.444 \\
\hline 90 & 8374 & 12766 & 0.506 \\
\hline 90 & 15941 & 24046 & 0.501 \\
\hline 90 & 19143 & 28867 & 0.500 \\
\hline 120 & 8526 & 14483 & 0.564 \\
\hline 120 & 20358 & 34381 & 0.560 \\
\hline 120 & 32899 & 55122 & 0.556 \\
\hline
\end{tabular}




\section{Order in 2-Iodothiophene, (1.0 equiv).}

(E)-1

2-Iodothiophene

Tetra- $n$-butylammonium fluoride

Bis(dibenzylideneacetone)palladium $2.5 \mathrm{mM}$ (0.05 equiv)
$50 \mathrm{mM}$ (1.0 equiv)

$50 \mathrm{mM}$ (1.0 equiv)

$188 \mathrm{mM}$ (3.75 equiv)

Following the General Procedure, silanol (E)-1 (43 mg, $0.25 \mathrm{mmol})$ and biphenyl (10.5 $\mathrm{mg}$ ) were dissolved in TBAF solution $(940 \mu \mathrm{L}, 1.0 \mathrm{M}$ in THF, $0.94 \mathrm{mmol})$ in a flame dried 2neck round-bottomed flask, equipped with a stir bar and a septum under argon. Dry THF (3.05 $\mathrm{mL}$ ) was added and the clear, slightly-yellow solution was immersed into a water-bath at $23{ }^{\circ} \mathrm{C} \pm$ $1{ }^{\circ} \mathrm{C}$ for $30 \mathrm{~min}$. The 2-iodothiophene $(28 \mu \mathrm{L}, 0.25 \mathrm{mmol})$ was then added and the reaction was stirred for $1 \mathrm{~min}$. A suspension of $\operatorname{Pd}(\mathrm{dba})_{2}(7.2 \mathrm{mg}, 0.0125 \mathrm{mmol})$ in dry THF $(1.0 \mathrm{~mL})$ was added. The initially purple, turbid reaction mixture turns into a clear solution within 30 seconds. Aliquots $(\sim 200 \mu \mathrm{L})$ were withdrawn after 1, 2, 3, 4, 5, 7, 10, 15, 20, 30, 40, 50, 60, 75, 90, and $120 \mathrm{~min}$. To quench, these samples were added to $100 \mu \mathrm{L}$ of a $20 \%$ aqueous solution of 2dimethylaminoethanethiol hydrochloride. The clear colorless to slightly-yellow solution was then filtered through a plug of silica gel, and the silica gel was washed with diethyl ether to give a total sample volume of $\sim 2 \mathrm{~mL}$. This sample was injected into the GC three times.

initial rate $=0.0094$

$\mathrm{k}_{\text {obs }}=0.0026$
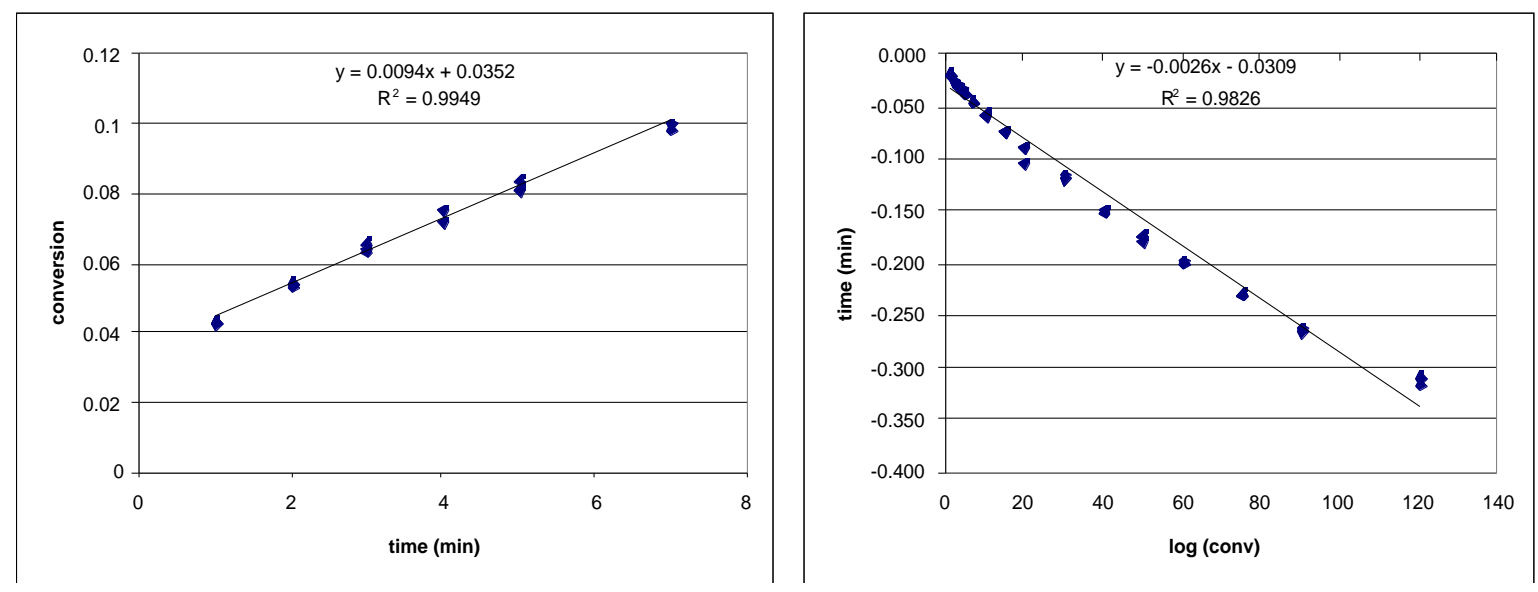


\begin{tabular}{|c|c|c|c|c|}
\hline time & area $\mathrm{A}$ & area 16 & conversion & $\log (1-\mathrm{conv})$ \\
\hline 1 & 6725 & 735 & 0.043 & -0.019 \\
\hline 1 & 7165 & 788 & 0.044 & -0.019 \\
\hline 1 & 8101 & 877 & 0.043 & -0.019 \\
\hline 2 & 5000 & 690 & 0.055 & -0.024 \\
\hline 2 & 5326 & 722 & 0.054 & -0.024 \\
\hline 2 & 5380 & 731 & 0.054 & -0.024 \\
\hline 3 & 6016 & 965 & 0.064 & -0.029 \\
\hline 3 & 6402 & 1041 & 0.064 & -0.029 \\
\hline 3 & 6682 & 1109 & 0.066 & -0.030 \\
\hline 4 & 5617 & 1073 & 0.076 & -0.034 \\
\hline 4 & 6496 & 1187 & 0.072 & -0.033 \\
\hline 5 & 8273 & 1756 & 0.084 & -0.038 \\
\hline 5 & 8892 & 1817 & 0.081 & -0.037 \\
\hline 5 & 9681 & 2002 & 0.082 & -0.037 \\
\hline 7 & 6705 & 1700 & 0.100 & -0.046 \\
\hline 7 & 7026 & 1753 & 0.099 & -0.045 \\
\hline 7 & 7151 & 1813 & 0.100 & -0.046 \\
\hline 10 & 6691 & 2104 & 0.125 & -0.058 \\
\hline 10 & 7282 & 2239 & 0.122 & -0.056 \\
\hline 10 & 7907 & 2487 & 0.125 & -0.058 \\
\hline 15 & 6182 & 2441 & 0.156 & -0.074 \\
\hline 15 & 6620 & 2585 & 0.155 & -0.073 \\
\hline 15 & 7229 & 2823 & 0.155 & -0.073 \\
\hline 20 & 7093 & 3815 & 0.213 & -0.104 \\
\hline 20 & 7695 & 3571 & 0.184 & -0.088 \\
\hline 20 & 8405 & 3904 & 0.184 & -0.088 \\
\hline 30 & 6900 & 4165 & 0.239 & -0.119 \\
\hline 30 & 7627 & 4434 & 0.230 & -0.114 \\
\hline 30 & 8816 & 5332 & 0.239 & -0.119 \\
\hline 40 & 6677 & 4881 & 0.289 & -0.148 \\
\hline 40 & 7292 & 5393 & 0.293 & -0.150 \\
\hline 40 & 8489 & 6237 & 0.291 & -0.149 \\
\hline 50 & 6209 & 5279 & 0.337 & -0.178 \\
\hline 50 & 7149 & 5918 & 0.328 & -0.173 \\
\hline 50 & 7404 & 6112 & 0.327 & -0.172 \\
\hline 60 & 7439 & 6857 & 0.365 & -0.197 \\
\hline 60 & 8409 & 7834 & 0.369 & -0.200 \\
\hline 60 & 8717 & 8078 & 0.367 & -0.199 \\
\hline 75 & 5147 & 5317 & 0.409 & -0.228 \\
\hline 75 & 5908 & 6107 & 0.409 & -0.229 \\
\hline 75 & 5766 & 5987 & 0.411 & -0.230 \\
\hline 90 & 6949 & 7942 & 0.453 & -0.262 \\
\hline 90 & 6313 & 7240 & 0.454 & -0.263 \\
\hline 90 & 7772 & 8963 & 0.457 & -0.265 \\
\hline 120 & 6152 & 8023 & 0.516 & -0.316 \\
\hline 120 & 6397 & 8256 & 0.511 & -0.311 \\
\hline 120 & 8107 & 10412 & 0.509 & -0.309 \\
\hline
\end{tabular}




\section{Order in 2-Iodothiophene, (1.0 equiv).}

(E)-1

2-Iodothiophene

Tetra- $n$-butylammonium fluoride

Bis(dibenzylideneacetone)palladium $2.5 \mathrm{mM}$ (0.05 equiv)
$50 \mathrm{mM}$ (1.0 equiv)

$50 \mathrm{mM}$ (1.0 equiv)

$188 \mathrm{mM}$ (3.75 equiv)

Following the General Procedure, silanol (E)-1 (43 mg, $0.25 \mathrm{mmol})$ and biphenyl (10.1 $\mathrm{mg}$ ) were dissolved in TBAF solution $(940 \mu \mathrm{L}, 1.0 \mathrm{M}$ in THF, $0.94 \mathrm{mmol})$ in a flame dried 2neck round-bottomed flask, equipped with a stir bar and a septum under argon. Dry THF (3.05 $\mathrm{mL}$ ) was added and the clear, slightly-yellow solution was immersed into a water-bath at $23{ }^{\circ} \mathrm{C} \pm$ $1{ }^{\circ} \mathrm{C}$ for $30 \mathrm{~min}$. The 2-iodothiophene $(28 \mu \mathrm{L}, 0.25 \mathrm{mmol})$ was then added and the reaction was stirred for $1 \mathrm{~min}$. A suspension of $\mathrm{Pd}(\mathrm{dba})_{2}(7.2 \mathrm{mg}, 0.0125 \mathrm{mmol})$ in dry THF $(1.0 \mathrm{~mL})$ was added. The initially purple, turbid reaction mixture turns into a clear solution within 30 seconds. Aliquots $(\sim 200 \mu \mathrm{L})$ were withdrawn after 1, 2, 3, 4, 5, 7, 10, 15, 20, 30, 40, 50, 60, 75, 90, and $120 \mathrm{~min}$. To quench, these samples were added to $100 \mu \mathrm{L}$ of a $20 \%$ aqueous solution of 2dimethylaminoethanethiol hydrochloride. The clear colorless to slightly-yellow solution was then filtered through a plug of silica gel, and the silica gel was washed with diethyl ether to give a total sample volume of $\sim 2 \mathrm{~mL}$. This sample was injected into the GC three times.

initial rate $=0.0112$

$\mathrm{k}_{\text {obs }}=0.0043$
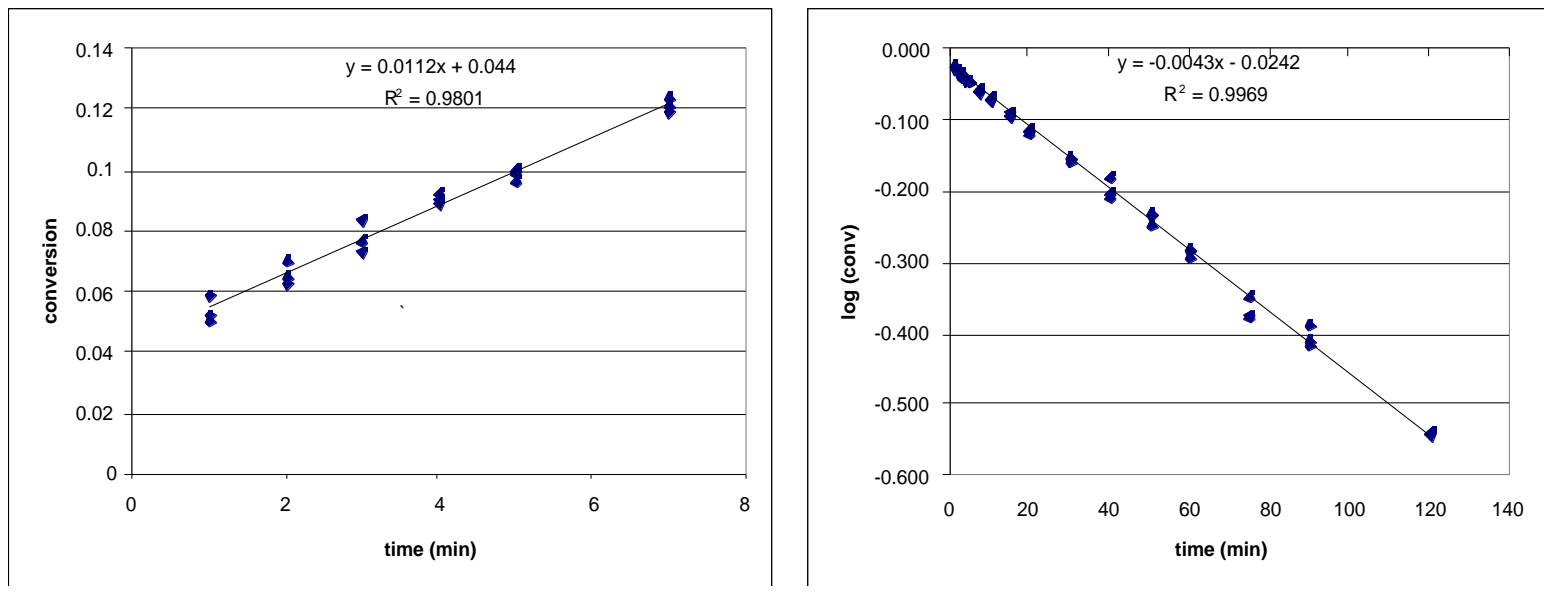


\begin{tabular}{|c|c|c|c|c|}
\hline time & area $\mathrm{A}$ & area 16 & conversion & $\log (1-\mathrm{conv})$ \\
\hline 1 & 4056 & 562 & 0.053 & -0.024 \\
\hline 1 & 4861 & 643 & 0.050 & -0.022 \\
\hline 1 & 5433 & 840 & 0.059 & -0.026 \\
\hline 2 & 4628 & 770 & 0.063 & -0.028 \\
\hline 2 & 7033 & 1200 & 0.065 & -0.029 \\
\hline 2 & 5746 & 1065 & 0.071 & -0.032 \\
\hline 3 & 4341 & 838 & 0.074 & -0.033 \\
\hline 3 & 6230 & 1263 & 0.077 & -0.035 \\
\hline 3 & 5007 & 1108 & 0.084 & -0.038 \\
\hline 4 & 3732 & 909 & 0.093 & -0.042 \\
\hline 4 & 5072 & 1205 & 0.090 & -0.041 \\
\hline 4 & 4594 & 1082 & 0.090 & -0.041 \\
\hline 5 & 4962 & 1259 & 0.097 & -0.044 \\
\hline 5 & 6751 & 1755 & 0.099 & -0.045 \\
\hline 5 & 6437 & 1702 & 0.101 & -0.046 \\
\hline 7 & 3118 & 1016 & 0.124 & -0.058 \\
\hline 7 & 4442 & 1417 & 0.122 & -0.056 \\
\hline 7 & 4348 & 1363 & 0.119 & -0.055 \\
\hline 10 & 3819 & 1478 & 0.147 & -0.069 \\
\hline 10 & 5586 & 2102 & 0.143 & -0.067 \\
\hline 10 & 5611 & 2176 & 0.148 & -0.069 \\
\hline 15 & 3477 & 1740 & 0.191 & -0.092 \\
\hline 15 & 4591 & 2189 & 0.182 & -0.087 \\
\hline 15 & 5281 & 2680 & 0.193 & -0.093 \\
\hline 20 & 4527 & 2712 & 0.228 & -0.112 \\
\hline 20 & 5840 & 3579 & 0.233 & -0.115 \\
\hline 20 & 5775 & 3630 & 0.239 & -0.119 \\
\hline 30 & 4171 & 3199 & 0.292 & -0.150 \\
\hline 30 & 5536 & 4375 & 0.301 & -0.156 \\
\hline 30 & 5632 & 4461 & 0.302 & -0.156 \\
\hline 40 & 4490 & 4014 & 0.341 & -0.181 \\
\hline 40 & 4761 & 4734 & 0.379 & -0.207 \\
\hline 40 & 4990 & 4854 & 0.371 & -0.201 \\
\hline 50 & 3884 & 4175 & 0.409 & -0.229 \\
\hline 50 & 4945 & 5382 & 0.415 & -0.233 \\
\hline 50 & 4910 & 5565 & 0.432 & -0.245 \\
\hline 60 & 4924 & 6160 & 0.477 & -0.281 \\
\hline 60 & 5922 & 7589 & 0.488 & -0.291 \\
\hline 60 & 6113 & 7703 & 0.480 & -0.284 \\
\hline 75 & 3942 & 5955 & 0.575 & -0.372 \\
\hline 75 & 4564 & 6935 & 0.579 & -0.375 \\
\hline 75 & 5161 & 7469 & 0.551 & -0.348 \\
\hline 90 & 4925 & 7884 & 0.610 & -0.409 \\
\hline 90 & 6181 & 9966 & 0.614 & -0.414 \\
\hline 90 & 5347 & 8279 & 0.590 & -0.387 \\
\hline 120 & 4737 & 8850 & 0.712 & -0.540 \\
\hline 120 & 6315 & 11773 & 0.710 & -0.538 \\
\hline
\end{tabular}




\section{Order in 2-Iodothiophene, (1.0 equiv).}

(E)-1

2-Iodothiophene

Tetra- $n$-butylammonium fluoride

Bis(dibenzylideneacetone)palladium $2.5 \mathrm{mM}$ (0.05 equiv)
$50 \mathrm{mM}$ (1.0 equiv)

$50 \mathrm{mM}$ (1.0 equiv)

$188 \mathrm{mM}$ (3.75 equiv)

Following the General Procedure, silanol (E)-1 (43 mg, $0.25 \mathrm{mmol})$ and biphenyl (5.8 $\mathrm{mg}$ ) were dissolved in TBAF solution $(940 \mu \mathrm{L}, 1.0 \mathrm{M}$ in THF, $0.94 \mathrm{mmol})$ in a flame dried 2neck round-bottomed flask, equipped with a stir bar and a septum under argon. Dry THF (3.05 $\mathrm{mL}$ ) was added and the clear, slightly-yellow solution was immersed into a water-bath at $23{ }^{\circ} \mathrm{C} \pm$ $1{ }^{\circ} \mathrm{C}$ for $30 \mathrm{~min}$. The 2-iodothiophene $(28 \mu \mathrm{L}, 0.25 \mathrm{mmol})$ was then added and the reaction was stirred for $1 \mathrm{~min}$. A suspension of $\mathrm{Pd}(\mathrm{dba})_{2}(7.2 \mathrm{mg}, 0.0125 \mathrm{mmol})$ in dry THF $(1.0 \mathrm{~mL})$ was added. The initially purple, turbid reaction mixture turns into a clear solution within 30 seconds. Aliquots $(\sim 200 \mu \mathrm{L})$ were withdrawn after 1, 2, 3, 4, 5, 7, 10, 15, 20, 30, 40, 50, 60, 75, 90, and $120 \mathrm{~min}$. To quench, these samples were added to $100 \mu \mathrm{L}$ of a $20 \%$ aqueous solution of 2dimethylaminoethanethiol hydrochloride. The clear colorless to slightly-yellow solution was then filtered through a plug of silica gel, and the silica gel was washed with diethyl ether to give a total sample volume of $\sim 2 \mathrm{~mL}$. This sample was injected into the GC three times.

initial rate $=0.0104$

$\mathrm{k}_{\text {obs }}=0.0029$
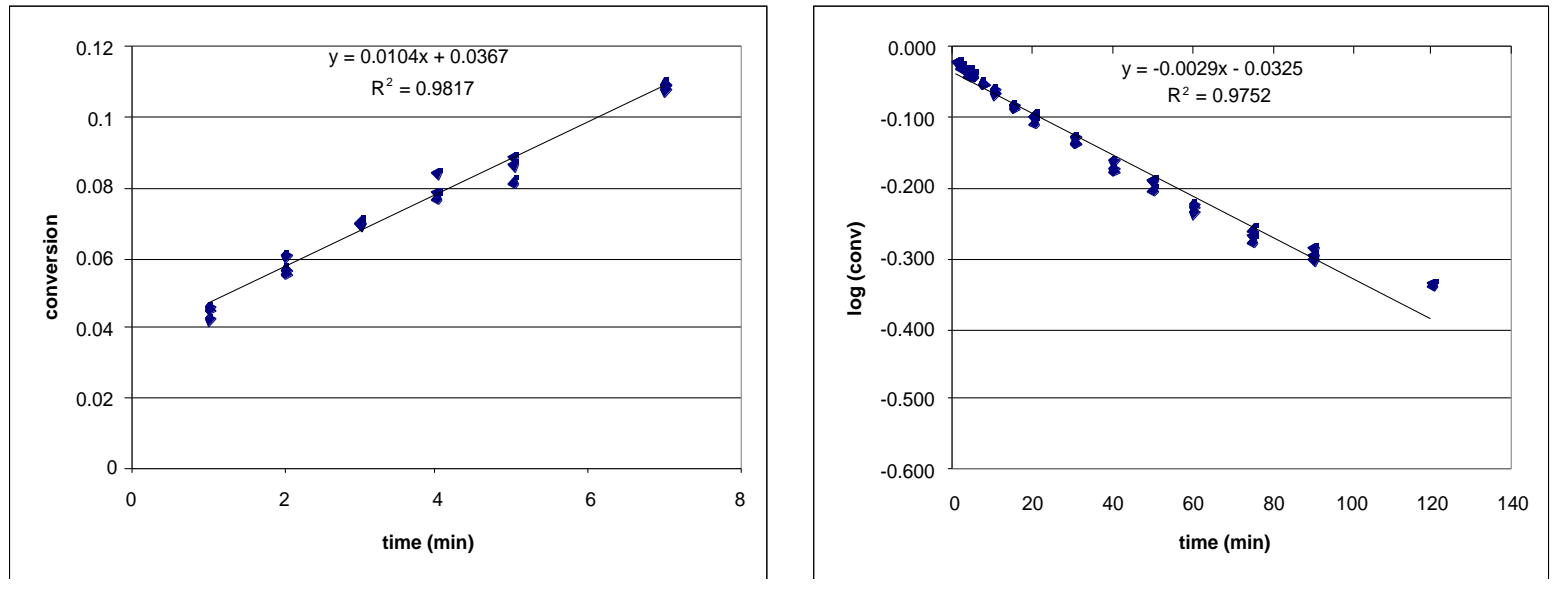


\begin{tabular}{|c|c|c|c|c|}
\hline time & area $\mathrm{A}$ & area $\mathbf{1 6}$ & conversion & $\log (1-\mathrm{conv})$ \\
\hline 1 & 3686 & 726 & 0.043 & -0.019 \\
\hline 1 & 3248 & 687 & 0.046 & -0.021 \\
\hline 1 & 3401 & 706 & 0.045 & -0.020 \\
\hline 2 & 3987 & 1037 & 0.057 & -0.025 \\
\hline 2 & 3608 & 920 & 0.056 & -0.025 \\
\hline 2 & 4021 & 1119 & 0.061 & -0.027 \\
\hline 3 & 4095 & 1315 & 0.070 & -0.032 \\
\hline 3 & 3709 & 1188 & 0.070 & -0.032 \\
\hline 3 & 4422 & 1411 & 0.070 & -0.031 \\
\hline 4 & 3620 & 1282 & 0.077 & -0.035 \\
\hline 4 & 3298 & 1275 & 0.085 & -0.038 \\
\hline 4 & 2904 & 1048 & 0.079 & -0.036 \\
\hline 5 & 3637 & 1484 & 0.089 & -0.041 \\
\hline 5 & 4109 & 1625 & 0.087 & -0.039 \\
\hline 5 & 3982 & 1492 & 0.082 & -0.037 \\
\hline 7 & 2962 & 1467 & 0.108 & -0.050 \\
\hline 7 & 3233 & 1621 & 0.110 & -0.050 \\
\hline 7 & 3603 & 1799 & 0.109 & -0.050 \\
\hline 10 & 3502 & 2047 & 0.128 & -0.059 \\
\hline 10 & 3750 & 2248 & 0.131 & -0.061 \\
\hline 10 & 3741 & 2316 & 0.135 & -0.063 \\
\hline 15 & 3841 & 2990 & 0.170 & -0.081 \\
\hline 15 & 4491 & 3460 & 0.169 & -0.080 \\
\hline 15 & 4500 & 3623 & 0.176 & -0.084 \\
\hline 20 & 3739 & 3419 & 0.200 & -0.097 \\
\hline 20 & 4147 & 3716 & 0.196 & -0.095 \\
\hline 20 & 4050 & 4042 & 0.218 & -0.107 \\
\hline 30 & 3330 & 4077 & 0.268 & -0.135 \\
\hline 30 & 3460 & 4004 & 0.253 & -0.127 \\
\hline 30 & 3554 & 4320 & 0.266 & -0.134 \\
\hline 40 & 3838 & 5813 & 0.331 & -0.175 \\
\hline 40 & 4273 & 6049 & 0.310 & -0.161 \\
\hline 40 & 4089 & 6070 & 0.325 & -0.171 \\
\hline 50 & 3572 & 5762 & 0.353 & -0.189 \\
\hline 50 & 4249 & 6847 & 0.352 & -0.189 \\
\hline 50 & 4372 & 7418 & 0.371 & -0.201 \\
\hline 60 & 4219 & 7782 & 0.403 & -0.224 \\
\hline 60 & 4489 & 8172 & 0.398 & -0.221 \\
\hline 60 & 4451 & 8471 & 0.416 & -0.234 \\
\hline 75 & 4109 & 8799 & 0.468 & -0.274 \\
\hline 75 & 4861 & 9962 & 0.448 & -0.258 \\
\hline 75 & 5558 & 11676 & 0.460 & -0.267 \\
\hline 90 & 5253 & 11809 & 0.492 & -0.294 \\
\hline 90 & 5764 & 12647 & 0.480 & -0.284 \\
\hline 90 & 5986 & 13694 & 0.500 & -0.301 \\
\hline 120 & 4130 & 10198 & 0.540 & -0.337 \\
\hline 120 & 4861 & 11931 & 0.537 & -0.334 \\
\hline
\end{tabular}




\section{Order in 2-Iodothiophene, 2.0 equiv.}

(E)-1

2-Iodothiophene

Tetra- $n$-butylammonium fluoride

Bis(dibenzylideneacetone)palladium $2.5 \mathrm{mM}$ (0.05 equiv)
$50 \mathrm{mM}$ (1.0 equiv)

$100 \mathrm{mM}$ (2.0 equiv)

$188 \mathrm{mM}$ (3.75 equiv)

Following the General Procedure, silanol (E)-1 (43 mg, $0.25 \mathrm{mmol})$ and biphenyl (14.7 $\mathrm{mg}$ ) were dissolved in TBAF solution $(940 \mu \mathrm{L}, 1.0 \mathrm{M}$ in THF, $0.94 \mathrm{mmol})$ in a flame dried 2neck round-bottomed flask, equipped with a stir bar and a septum under argon. Dry THF (3.0 $\mathrm{mL}$ ) was added and the clear, slightly-yellow solution was immersed into a water-bath at $23{ }^{\circ} \mathrm{C} \pm$ $1{ }^{\circ} \mathrm{C}$ for $30 \mathrm{~min}$. The 2 -iodothiophene $(56 \mu \mathrm{L}, 0.5 \mathrm{mmol})$ was then added and the reaction was stirred for $1 \mathrm{~min}$. A suspension of $\operatorname{Pd}(\mathrm{dba})_{2}(7.2 \mathrm{mg}, 0.0125 \mathrm{mmol})$ in dry THF $(1.0 \mathrm{~mL})$ was added. The initially purple, turbid reaction mixture turns into a clear solution within 30 seconds. Aliquots $(\sim 200 \mu \mathrm{L})$ were withdrawn after 1, 2, 3, 4, 5, 7, 10, 15, 20, 30, 40, 50, 60, 75, 90, and $120 \mathrm{~min}$. To quench, these samples were added to $100 \mu \mathrm{L}$ of a $20 \%$ aqueous solution of 2dimethylaminoethanethiol hydrochloride. The clear colorless to slightly-yellow solution was then filtered through a plug of silica gel, and the silica gel was washed with diethyl ether to give a total sample volume of $\sim 2 \mathrm{~mL}$. This sample was injected into the GC three times.

initial rate $=0.0156$

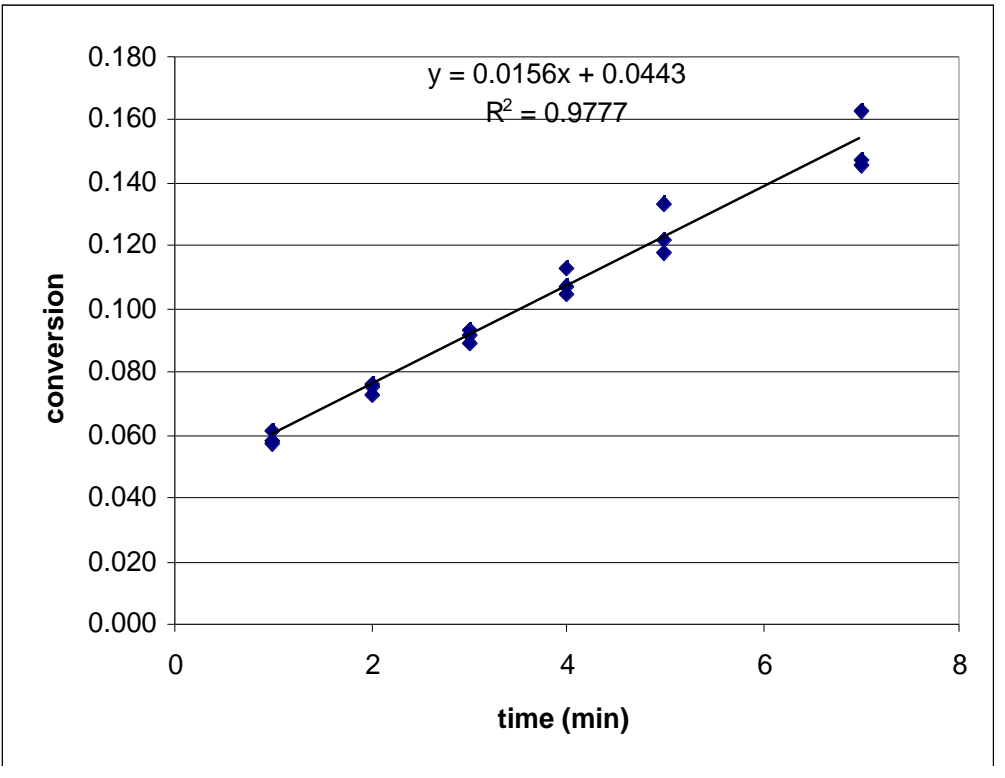




\begin{tabular}{|c|c|c|c|}
\hline time & area standard & area 16 & conversion \\
\hline 1 & 5541 & 571 & 0.057 \\
\hline 1 & 5642 & 592 & 0.058 \\
\hline 1 & 7011 & 780 & 0.062 \\
\hline 2 & 6005 & 818 & 0.076 \\
\hline 2 & 6027 & 829 & 0.076 \\
\hline 2 & 6511 & 859 & 0.073 \\
\hline 3 & 5159 & 870 & 0.093 \\
\hline 3 & 5297 & 875 & 0.092 \\
\hline 3 & 6518 & 1053 & 0.090 \\
\hline 4 & 5893 & 1135 & 0.107 \\
\hline 4 & 5943 & 1122 & 0.105 \\
\hline 4 & 6993 & 1423 & 0.113 \\
\hline 5 & 6003 & 1276 & 0.118 \\
\hline 5 & 6726 & 1480 & 0.122 \\
\hline 5 & 7498 & 1807 & 0.134 \\
\hline 7 & 5018 & 1320 & 0.146 \\
\hline 7 & 5665 & 1507 & 0.147 \\
\hline 7 & 5847 & 1713 & 0.162 \\
\hline 10 & 3955 & 1250 & 0.175 \\
\hline 10 & 5341 & 1749 & 0.182 \\
\hline 10 & 5911 & 1866 & 0.175 \\
\hline 15 & 6372 & 2571 & 0.224 \\
\hline 15 & 7336 & 2981 & 0.225 \\
\hline 15 & 7987 & 3141 & 0.218 \\
\hline 20 & 7063 & 3454 & 0.271 \\
\hline 20 & 8300 & 4005 & 0.268 \\
\hline 20 & 9139 & 4462 & 0.271 \\
\hline 30 & 5803 & 3810 & 0.364 \\
\hline 30 & 7057 & 4516 & 0.355 \\
\hline 30 & 7678 & 4974 & 0.359 \\
\hline 40 & 7829 & 6169 & 0.437 \\
\hline 40 & 9432 & 7388 & 0.434 \\
\hline 40 & 10872 & 8420 & 0.429 \\
\hline 50 & 5085 & 4634 & 0.505 \\
\hline 50 & 6665 & 6154 & 0.512 \\
\hline 50 & 7261 & 6609 & 0.505 \\
\hline 60 & 5720 & 5909 & 0.573 \\
\hline 60 & 6662 & 6800 & 0.566 \\
\hline 60 & 7569 & 7419 & 0.543 \\
\hline 75 & 5961 & 6953 & 0.647 \\
\hline 75 & 6772 & 7741 & 0.634 \\
\hline 75 & 7984 & 9128 & 0.634 \\
\hline 90 & 7059 & 9026 & 0.709 \\
\hline 90 & 7319 & 9459 & 0.716 \\
\hline 90 & 8982 & 11262 & 0.695 \\
\hline 120 & 6692 & 9496 & 0.787 \\
\hline 120 & 7737 & 11019 & 0.790 \\
\hline
\end{tabular}




\section{Order in 2-Iodothiophene, 2.0 equiv.}

(E)-1

2-Iodothiophene

Tetra- $n$-butylammonium fluoride

Bis(dibenzylideneacetone)palladium $2.5 \mathrm{mM}$ (0.05 equiv)
$50 \mathrm{mM}$ (1.0 equiv)

$100 \mathrm{mM}$ (2.0 equiv)

$188 \mathrm{mM}$ (3.75 equiv)

Following the General Procedure, silanol (E)-1 (43 mg, $0.25 \mathrm{mmol})$ and biphenyl $(9.5$ $\mathrm{mg}$ ) were dissolved in TBAF solution $(940 \mu \mathrm{L}, 1.0 \mathrm{M}$ in THF, $0.94 \mathrm{mmol})$ in a flame dried 2neck round-bottomed flask, equipped with a stir bar and a septum under argon. Dry THF (3.0 $\mathrm{mL}$ ) was added and the clear, slightly-yellow solution was immersed into a water-bath at $23{ }^{\circ} \mathrm{C} \pm$ $1{ }^{\circ} \mathrm{C}$ for $30 \mathrm{~min}$. The 2 -iodothiophene $(56 \mu \mathrm{L}, 0.5 \mathrm{mmol})$ was then added and the reaction was stirred for $1 \mathrm{~min}$. A suspension of $\operatorname{Pd}(\mathrm{dba})_{2}(7.2 \mathrm{mg}, 0.0125 \mathrm{mmol})$ in dry THF $(1.0 \mathrm{~mL})$ was added. The initially purple, turbid reaction mixture turns into a clear solution within 30 seconds. Aliquots $(\sim 200 \mu \mathrm{L})$ were withdrawn after 1, 2, 3, 4, 5, 7, 10, 15, 20, 30, 40, 50, 60, 75, 90, and $120 \mathrm{~min}$. To quench, these samples were added to $100 \mu \mathrm{L}$ of a $20 \%$ aqueous solution of 2dimethylaminoethanethiol hydrochloride. The clear colorless to slightly-yellow solution was then filtered through a plug of silica gel, and the silica gel was washed with diethyl ether to give a total sample volume of $\sim 2 \mathrm{~mL}$. This sample was injected into the GC three times.

initial rate $=0.011$

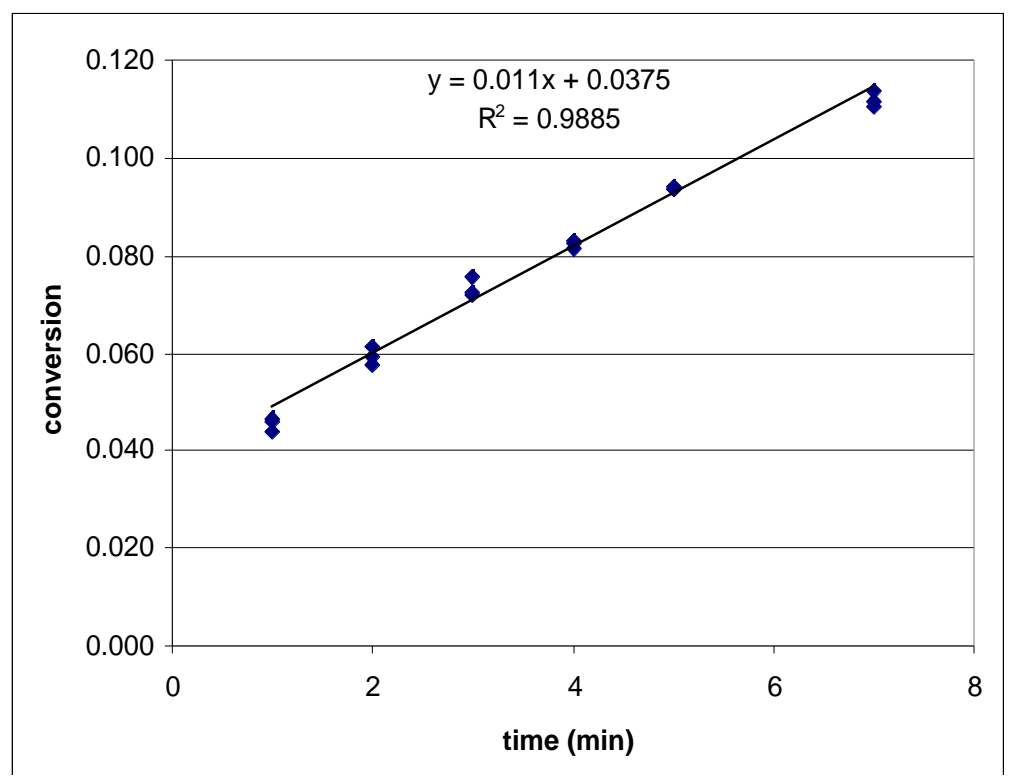




\begin{tabular}{|c|c|c|c|}
\hline time & area $\mathrm{A}$ & area 16 & conversion \\
\hline 1 & 7301 & 939 & 0.046 \\
\hline 1 & 9701 & 1256 & 0.046 \\
\hline 1 & 11716 & 1439 & 0.044 \\
\hline 2 & 7117 & 1213 & 0.061 \\
\hline 2 & 7781 & 1256 & 0.058 \\
\hline 2 & 10965 & 1819 & 0.059 \\
\hline 3 & 8952 & 1812 & 0.073 \\
\hline 3 & 13709 & 2886 & 0.075 \\
\hline 3 & 15841 & 3176 & 0.072 \\
\hline 4 & 11570 & 2671 & 0.083 \\
\hline 4 & 11217 & 2596 & 0.083 \\
\hline 4 & 12705 & 2890 & 0.081 \\
\hline 5 & 13905 & 3622 & 0.093 \\
\hline 5 & 14201 & 3721 & 0.094 \\
\hline 5 & 14379 & 3751 & 0.093 \\
\hline 7 & 4574 & 1409 & 0.110 \\
\hline 7 & 6387 & 2022 & 0.113 \\
\hline 7 & 7936 & 2475 & 0.112 \\
\hline 10 & 5228 & 1980 & 0.136 \\
\hline 10 & 5737 & 2234 & 0.140 \\
\hline 10 & 6095 & 2294 & 0.135 \\
\hline 15 & 5117 & 2533 & 0.177 \\
\hline 15 & 4887 & 2454 & 0.180 \\
\hline 15 & 6632 & 3306 & 0.179 \\
\hline 20 & 6143 & 3675 & 0.214 \\
\hline 20 & 7158 & 4402 & 0.220 \\
\hline 20 & 7577 & 4586 & 0.217 \\
\hline 30 & 8383 & 6757 & 0.289 \\
\hline 30 & 17264 & 13929 & 0.289 \\
\hline 30 & 22352 & 17438 & 0.280 \\
\hline 40 & 5356 & 5318 & 0.356 \\
\hline 40 & 14611 & 14503 & 0.356 \\
\hline 40 & 21781 & 21030 & 0.346 \\
\hline 50 & 6552 & 7569 & 0.414 \\
\hline 50 & 21580 & 24793 & 0.412 \\
\hline 50 & 32564 & 36980 & 0.407 \\
\hline 60 & 5833 & 7592 & 0.466 \\
\hline 60 & 17298 & 22454 & 0.465 \\
\hline 60 & 36142 & 46295 & 0.459 \\
\hline 75 & 6207 & 9297 & 0.537 \\
\hline 75 & 20813 & 31088 & 0.535 \\
\hline 75 & 24123 & 35500 & 0.527 \\
\hline 90 & 6022 & 10038 & 0.597 \\
\hline 90 & 17652 & 29216 & 0.593 \\
\hline 90 & 16872 & 28081 & 0.596 \\
\hline 120 & 6005 & 11464 & 0.684 \\
\hline 120 & 25671 & 49041 & 0.684 \\
\hline
\end{tabular}




\section{Order in 2-Iodothiophene, 2.0 equiv.}

(E)-1

2-Iodothiophene

Tetra- $n$-butylammonium fluoride

Bis(dibenzylideneacetone)palladium $2.5 \mathrm{mM}$ (0.05 equiv)
$50 \mathrm{mM}$ (1.0 equiv)

$100 \mathrm{mM}$ (2.0 equiv)

$188 \mathrm{mM}$ (3.75 equiv)

Following the General Procedure, silanol (E)-1 (43 mg, $0.25 \mathrm{mmol})$ and biphenyl (11.1 $\mathrm{mg}$ ) were dissolved in TBAF solution $(940 \mu \mathrm{L}, 1.0 \mathrm{M}$ in THF, $0.94 \mathrm{mmol})$ in a flame dried 2neck round-bottomed flask, equipped with a stir bar and a septum under argon. Dry THF (3.0 $\mathrm{mL}$ ) was added and the clear, slightly-yellow solution was immersed into a water-bath at $23{ }^{\circ} \mathrm{C} \pm$ $1{ }^{\circ} \mathrm{C}$ for $30 \mathrm{~min}$. The 2 -iodothiophene $(56 \mu \mathrm{L}, 0.5 \mathrm{mmol})$ was then added and the reaction was stirred for $1 \mathrm{~min}$. A suspension of $\operatorname{Pd}(\mathrm{dba})_{2}(7.2 \mathrm{mg}, 0.0125 \mathrm{mmol})$ in dry THF $(1.0 \mathrm{~mL})$ was added. The initially purple, turbid reaction mixture turns into a clear solution within 30 seconds. Aliquots $(\sim 200 \mu \mathrm{L})$ were withdrawn after 1, 2, 3, 4, 5, 7, 10, 15, 20, 30, 40, 50, 60, 75, 90, and $120 \mathrm{~min}$. To quench, these samples were added to $100 \mu \mathrm{L}$ of a $20 \%$ aqueous solution of 2dimethylaminoethanethiol hydrochloride. The clear colorless to slightly-yellow solution was then filtered through a plug of silica gel, and the silica gel was washed with diethyl ether to give a total sample volume of $\sim 2 \mathrm{~mL}$. This sample was injected into the GC three times.

initial rate $=0.0099$

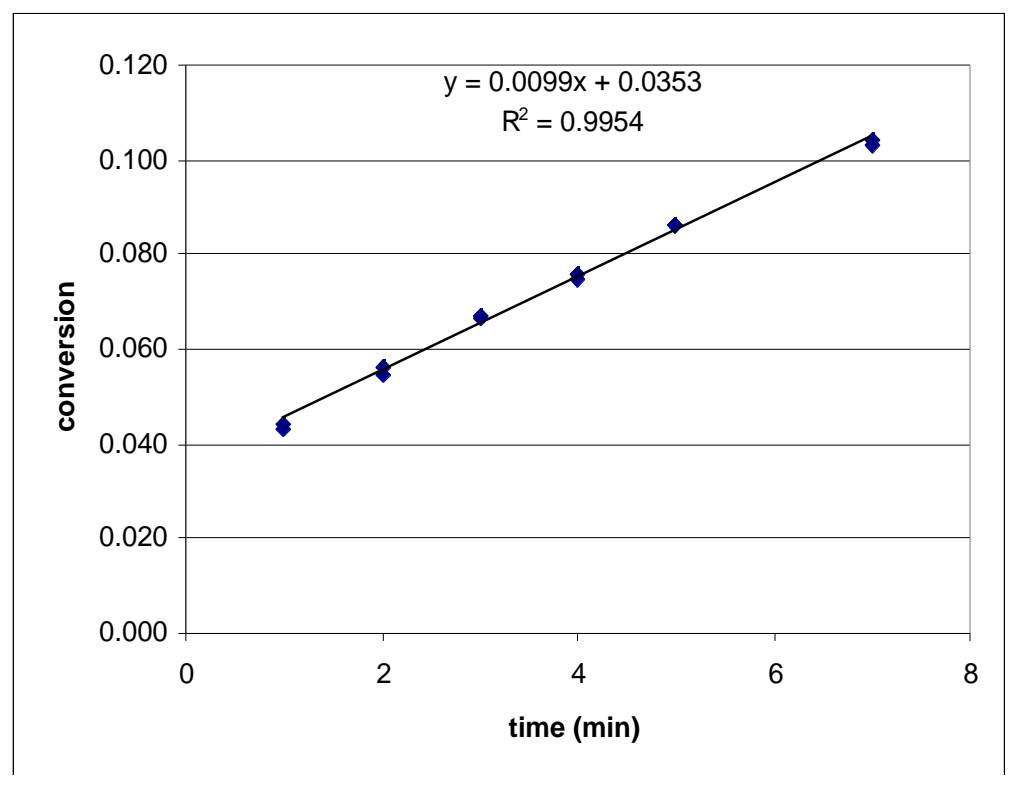




\begin{tabular}{|c|c|c|c|}
\hline time & area $\mathrm{A}$ & area 16 & conversion \\
\hline 1 & 19496 & 2056 & 0.044 \\
\hline 1 & 47575 & 4887 & 0.043 \\
\hline 2 & 19707 & 2641 & 0.056 \\
\hline 2 & 30994 & 4050 & 0.055 \\
\hline 3 & 22503 & 3615 & 0.067 \\
\hline 3 & 57581 & 9132 & 0.066 \\
\hline 4 & 16125 & 2915 & 0.076 \\
\hline 4 & 37384 & 6680 & 0.075 \\
\hline 5 & 14824 & 3058 & 0.086 \\
\hline 5 & 33119 & 6802 & 0.086 \\
\hline 7 & 8947 & 2200 & 0.103 \\
\hline 7 & 23451 & 5828 & 0.104 \\
\hline 10 & 12933 & 3906 & 0.126 \\
\hline 10 & 35531 & 10819 & 0.127 \\
\hline 15 & 9376 & 3728 & 0.166 \\
\hline 15 & 21682 & 8480 & 0.164 \\
\hline 20 & 9073 & 4308 & 0.199 \\
\hline 20 & 20229 & 9668 & 0.200 \\
\hline 30 & 5832 & 3738 & 0.268 \\
\hline 30 & 20263 & 12888 & 0.266 \\
\hline 40 & 9041 & 7240 & 0.335 \\
\hline 40 & 32634 & 26010 & 0.334 \\
\hline 50 & 7998 & 7185 & 0.376 \\
\hline 50 & 27247 & 25205 & 0.387 \\
\hline 60 & 7995 & 8471 & 0.444 \\
\hline 60 & 25288 & 26505 & 0.439 \\
\hline 75 & 10401 & 12798 & 0.515 \\
\hline 75 & 29074 & 35598 & 0.513 \\
\hline 90 & 9933 & 13775 & 0.581 \\
\hline 90 & 31480 & 43141 & 0.574 \\
\hline 120 & 11720 & 18870 & 0.674 \\
\hline 120 & 33272 & 52790 & 0.664 \\
\hline
\end{tabular}




\section{Order in Palladium, (0.025 equiv).}

(E)-1

2-Iodothiophene

Tetra- $n$-butylammonium fluoride

Bis(dibenzylideneacetone)palladium
$50 \mathrm{mM}$ (1.0 equiv)

$50 \mathrm{mM}$ (1.0 equiv)

$188 \mathrm{mM}$ (3.75 equiv)

$1.25 \mathrm{mM}$ ( 0.025 equiv $)$

Following the General Procedure, silanol (E)-1 (43 mg, $0.25 \mathrm{mmol})$ and biphenyl (22.1 $\mathrm{mg}$ ) were dissolved in TBAF solution $(940 \mu \mathrm{L}, 1.0 \mathrm{M}$ in THF, $0.94 \mathrm{mmol})$ in a flame dried 2neck round-bottomed flask, equipped with a stir bar and a septum under argon. Dry THF (3.0 $\mathrm{mL}$ ) was added and the clear, slightly-yellow solution was immersed into a water-bath at $23{ }^{\circ} \mathrm{C} \pm$ $1{ }^{\circ} \mathrm{C}$ for $30 \mathrm{~min}$. The 2-iodothiophene $(28 \mu \mathrm{L}, 0.25 \mathrm{mmol})$ was then added and the reaction was stirred for $1 \mathrm{~min}$. A suspension of $\mathrm{Pd}(\mathrm{dba})_{2}(3.6 \mathrm{mg}, 0.00625 \mathrm{mmol})$ in dry THF $(1.0 \mathrm{~mL})$ was added. The initially purple, turbid reaction mixture turns into a clear solution within 30 seconds. Aliquots $(\sim 200 \mu \mathrm{L})$ were withdrawn after 1, 2, 3, 4, 5, 7, 10, 15, 20, 30, 40, 50, 60, 75, 90, and $120 \mathrm{~min}$. To quench, these samples were added to $100 \mu \mathrm{L}$ of a $20 \%$ aqueous solution of 2dimethylaminoethanethiol hydrochloride. The clear colorless to slightly-yellow solution was then filtered through a plug of silica gel, and the silica gel was washed with diethyl ether to give a total sample volume of $\sim 2 \mathrm{~mL}$. This sample was injected into the GC three times.

$\mathrm{k}_{\text {obs }}=0.002$

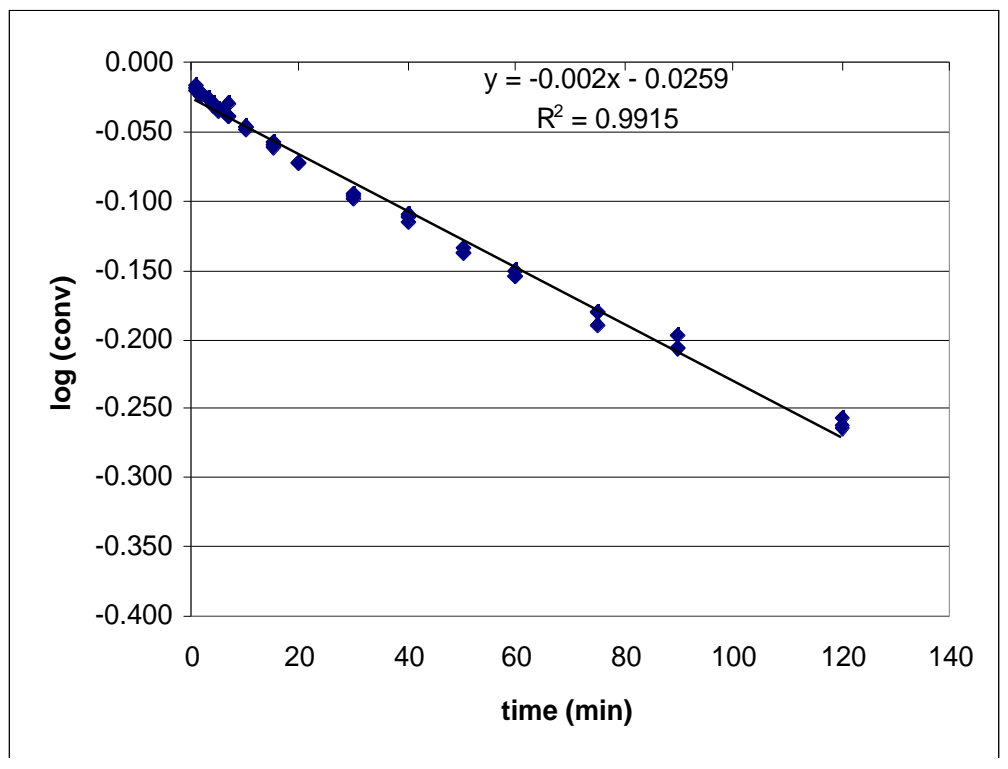




\begin{tabular}{|c|c|c|c|c|}
\hline Time & $\operatorname{area} \mathrm{A}$ & area $\mathbf{1 6}$ & conversion & $\log (1-\mathrm{conv})$ \\
\hline 1 & 10056 & 539 & 0.045 & -0.020 \\
\hline 1 & 10686 & 539 & 0.042 & -0.019 \\
\hline 1 & 11387 & 528 & 0.039 & -0.017 \\
\hline 2 & 7775 & 489 & 0.052 & -0.023 \\
\hline 2 & 9348 & 547 & 0.049 & -0.022 \\
\hline 2 & 9606 & 586 & 0.051 & -0.023 \\
\hline 3 & 6078 & 439 & 0.060 & -0.027 \\
\hline 4 & 8687 & 714 & 0.069 & -0.031 \\
\hline 4 & 8986 & 732 & 0.068 & -0.031 \\
\hline 5 & 6930 & 626 & 0.075 & -0.034 \\
\hline 5 & 7640 & 680 & 0.074 & -0.033 \\
\hline 5 & 7752 & 709 & 0.076 & -0.034 \\
\hline 7 & 9929 & 773 & 0.065 & -0.029 \\
\hline 7 & 10855 & 1114 & 0.086 & -0.039 \\
\hline 7 & 12557 & 1302 & 0.086 & -0.039 \\
\hline 10 & 7700 & 939 & 0.102 & -0.047 \\
\hline 10 & 8921 & 1127 & 0.105 & -0.048 \\
\hline 10 & 8155 & 1009 & 0.103 & -0.047 \\
\hline 15 & 7250 & 1079 & 0.124 & -0.058 \\
\hline 15 & 9097 & 1443 & 0.132 & -0.062 \\
\hline 15 & 8777 & 1365 & 0.130 & -0.060 \\
\hline 20 & 9237 & 1689 & 0.152 & -0.072 \\
\hline 20 & 10729 & 1977 & 0.154 & -0.072 \\
\hline 20 & 10183 & 1877 & 0.154 & -0.072 \\
\hline 30 & 7264 & 1780 & 0.204 & -0.099 \\
\hline 30 & 9038 & 2162 & 0.199 & -0.097 \\
\hline 30 & 8294 & 1944 & 0.195 & -0.094 \\
\hline 40 & 8661 & 2410 & 0.232 & -0.115 \\
\hline 40 & 10183 & 2784 & 0.228 & -0.112 \\
\hline 40 & 9299 & 2483 & 0.223 & -0.109 \\
\hline 50 & 5786 & 1883 & 0.271 & -0.137 \\
\hline 50 & 6559 & 2137 & 0.272 & -0.138 \\
\hline 50 & 6990 & 2224 & 0.265 & -0.134 \\
\hline 60 & 7667 & 2708 & 0.294 & -0.151 \\
\hline 60 & 8344 & 2998 & 0.299 & -0.155 \\
\hline 60 & 8774 & 3164 & 0.301 & -0.155 \\
\hline 75 & 9697 & 4131 & 0.355 & -0.190 \\
\hline 75 & 11573 & 4739 & 0.341 & -0.181 \\
\hline 75 & 10899 & 4434 & 0.339 & -0.180 \\
\hline 90 & 6353 & 2880 & 0.378 & -0.206 \\
\hline 90 & 8131 & 3687 & 0.378 & -0.206 \\
\hline 90 & 7251 & 3186 & 0.366 & -0.198 \\
\hline 120 & 4454 & 2427 & 0.454 & -0.263 \\
\hline 120 & 5089 & 2729 & 0.447 & -0.257 \\
\hline 120 & 5523 & 3018 & 0.455 & -0.264 \\
\hline
\end{tabular}




\section{Order in Palladium, (0.025 equiv).}

(E)-1

2-Iodothiophene

Tetra- $n$-butylammonium fluoride

Bis(dibenzylideneacetone)palladium
$50 \mathrm{mM}$ (1.0 equiv)

$50 \mathrm{mM}$ (1.0 equiv)

$188 \mathrm{mM}$ (3.75 equiv)

$1.25 \mathrm{mM}$ ( 0.025 equiv $)$

Following the General Procedure, silanol (E)-1 (43 mg, $0.25 \mathrm{mmol})$ and biphenyl (20.0 $\mathrm{mg}$ ) were dissolved in TBAF solution $(940 \mu \mathrm{L}, 1.0 \mathrm{M}$ in THF, $0.94 \mathrm{mmol})$ in a flame dried 2neck round-bottomed flask, equipped with a stir bar and a septum under argon. Dry THF (3.0 $\mathrm{mL}$ ) was added and the clear, slightly-yellow solution was immersed into a water-bath at $23{ }^{\circ} \mathrm{C} \pm$ $1{ }^{\circ} \mathrm{C}$ for $30 \mathrm{~min}$. The 2-iodothiophene $(28 \mu \mathrm{L}, 0.25 \mathrm{mmol})$ was then added and the reaction was stirred for $1 \mathrm{~min}$. A suspension of $\mathrm{Pd}(\mathrm{dba})_{2}(3.6 \mathrm{mg}, 0.00625 \mathrm{mmol})$ in dry THF $(1.0 \mathrm{~mL})$ was added. The initially purple, turbid reaction mixture turns into a clear solution within 30 seconds. Aliquots $(\sim 200 \mu \mathrm{L})$ were withdrawn after 1, 2, 3, 4, 5, 7, 10, 15, 20, 30, 40, 50, 60, 75, 90, and $120 \mathrm{~min}$. To quench, these samples were added to $100 \mu \mathrm{L}$ of a $20 \%$ aqueous solution of 2dimethylaminoethanethiol hydrochloride. The clear colorless to slightly-yellow solution was then filtered through a plug of silica gel, and the silica gel was washed with diethyl ether to give a total sample volume of $\sim 2 \mathrm{~mL}$. This sample was injected into the GC three times.

$\mathrm{k}_{\mathrm{obs}}=0.0022$

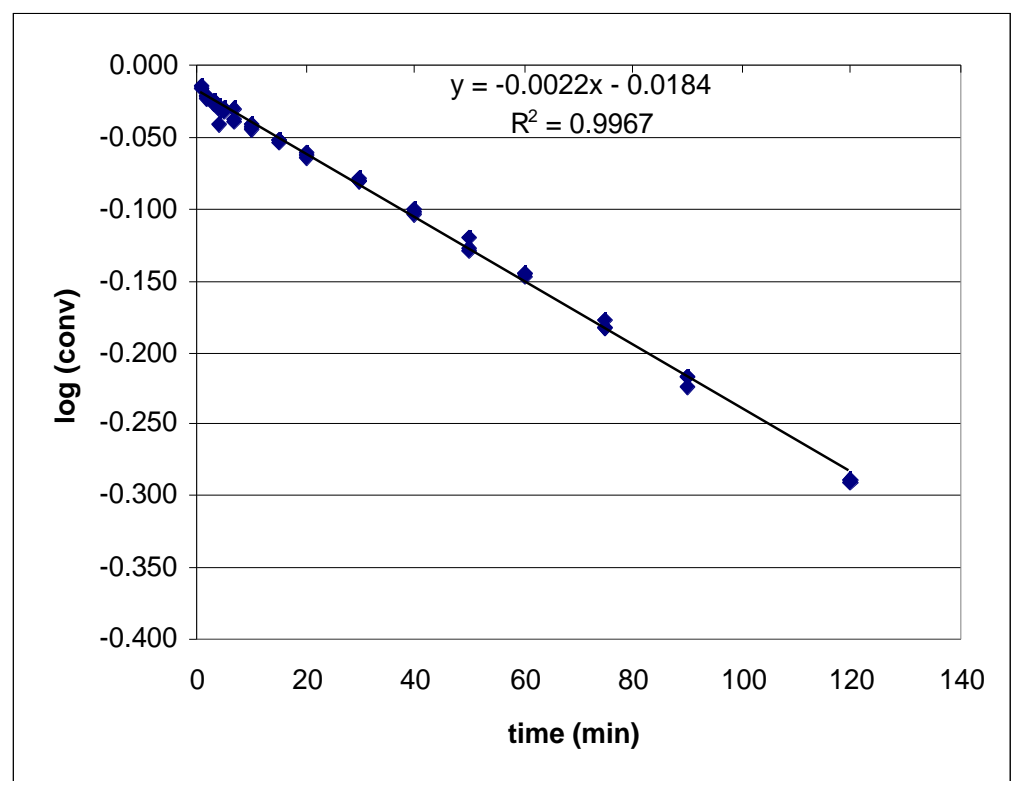




\begin{tabular}{|c|c|c|c|c|}
\hline time & area $\mathrm{A}$ & area $\mathbf{1 6}$ & conversion & $\log (1-\mathrm{conv})$ \\
\hline 1 & 11127 & 628 & 0.043 & -0.019 \\
\hline 1 & 10103 & 504 & 0.038 & -0.017 \\
\hline 1 & 13029 & 559 & 0.032 & -0.014 \\
\hline 2 & 9127 & 583 & 0.048 & -0.021 \\
\hline 2 & 9408 & 652 & 0.052 & -0.023 \\
\hline 2 & 10546 & 674 & 0.048 & -0.021 \\
\hline 3 & 9065 & 695 & 0.058 & -0.026 \\
\hline 3 & 9552 & 768 & 0.061 & -0.027 \\
\hline 3 & 11392 & 849 & 0.056 & -0.025 \\
\hline 4 & 9281 & 1130 & 0.092 & -0.042 \\
\hline 4 & 9233 & 813 & 0.066 & -0.030 \\
\hline 4 & 10962 & 941 & 0.065 & -0.029 \\
\hline 5 & 8946 & 837 & 0.071 & -0.032 \\
\hline 5 & 10231 & 937 & 0.069 & -0.031 \\
\hline 5 & 11592 & 1063 & 0.069 & -0.031 \\
\hline 7 & 4088 & 445 & 0.082 & -0.037 \\
\hline 7 & 4767 & 542 & 0.086 & -0.039 \\
\hline 7 & 5676 & 513 & 0.068 & -0.031 \\
\hline 10 & 5016 & 660 & 0.099 & -0.045 \\
\hline 10 & 5281 & 649 & 0.093 & -0.042 \\
\hline 10 & 6932 & 848 & 0.092 & -0.042 \\
\hline 15 & 5858 & 873 & 0.112 & -0.052 \\
\hline 15 & 6636 & 1011 & 0.115 & -0.053 \\
\hline 15 & 8193 & 1237 & 0.114 & -0.053 \\
\hline 20 & 6538 & 1156 & 0.133 & -0.062 \\
\hline 20 & 6858 & 1244 & 0.137 & -0.064 \\
\hline 20 & 8090 & 1422 & 0.133 & -0.062 \\
\hline 30 & 5621 & 1250 & 0.168 & -0.080 \\
\hline 30 & 5863 & 1313 & 0.169 & -0.080 \\
\hline 30 & 7405 & 1663 & 0.169 & -0.081 \\
\hline 40 & 6438 & 1808 & 0.212 & -0.103 \\
\hline 40 & 7452 & 2088 & 0.211 & -0.103 \\
\hline 40 & 9595 & 2616 & 0.206 & -0.100 \\
\hline 50 & 5183 & 1745 & 0.254 & -0.127 \\
\hline 50 & 5535 & 1772 & 0.241 & -0.120 \\
\hline 50 & 7416 & 2525 & 0.257 & -0.129 \\
\hline 60 & 4598 & 1759 & 0.289 & -0.148 \\
\hline 60 & 5097 & 1929 & 0.285 & -0.146 \\
\hline 60 & 6642 & 2506 & 0.285 & -0.145 \\
\hline 75 & 5297 & 2422 & 0.345 & -0.184 \\
\hline 75 & 5582 & 2491 & 0.337 & -0.178 \\
\hline 75 & 7369 & 3364 & 0.344 & -0.183 \\
\hline 90 & 4976 & 2659 & 0.403 & -0.224 \\
\hline 90 & 5302 & 2763 & 0.393 & -0.217 \\
\hline 90 & 7039 & 3670 & 0.393 & -0.217 \\
\hline 120 & 7611 & 4902 & 0.486 & -0.289 \\
\hline 120 & 10053 & 6510 & 0.488 & -0.291 \\
\hline
\end{tabular}




\section{Order in Palladium, (0.025 equiv).}

(E)-1

2-Iodothiophene

Tetra- $n$-butylammonium fluoride

Bis(dibenzylideneacetone)palladium
$50 \mathrm{mM}$ (1.0 equiv)

$50 \mathrm{mM}$ (1.0 equiv)

$188 \mathrm{mM}$ (3.75 equiv)

$1.25 \mathrm{mM}$ ( 0.025 equiv $)$

Following the General Procedure, silanol (E)-1 (43 mg, $0.25 \mathrm{mmol})$ and biphenyl (22.1 $\mathrm{mg}$ ) were dissolved in TBAF solution $(940 \mu \mathrm{L}, 1.0 \mathrm{M}$ in THF, $0.94 \mathrm{mmol})$ in a flame dried 2neck round-bottomed flask, equipped with a stir bar and a septum under argon. Dry THF (3.0 $\mathrm{mL}$ ) was added and the clear, slightly-yellow solution was immersed into a water-bath at $23{ }^{\circ} \mathrm{C} \pm$ $1{ }^{\circ} \mathrm{C}$ for $30 \mathrm{~min}$. The 2-iodothiophene $(28 \mu \mathrm{L}, 0.25 \mathrm{mmol})$ was then added and the reaction was stirred for $1 \mathrm{~min}$. A suspension of $\mathrm{Pd}(\mathrm{dba})_{2}(3.6 \mathrm{mg}, 0.00625 \mathrm{mmol})$ in dry THF $(1.0 \mathrm{~mL})$ was added. The initially purple, turbid reaction mixture turns into a clear solution within 30 seconds. Aliquots $(\sim 200 \mu \mathrm{L})$ were withdrawn after 1, 2, 3, 4, 5, 7, 10, 15, 20, 30, 40, 50, 60, 75, 90, and $120 \mathrm{~min}$. To quench, these samples were added to $100 \mu \mathrm{L}$ of a $20 \%$ aqueous solution of 2dimethylaminoethanethiol hydrochloride. The clear colorless to slightly-yellow solution was then filtered through a plug of silica gel, and the silica gel was washed with diethyl ether to give a total sample volume of $\sim 2 \mathrm{~mL}$. This sample was injected into the GC three times.

$\mathrm{k}_{\text {obs }}=0.002$

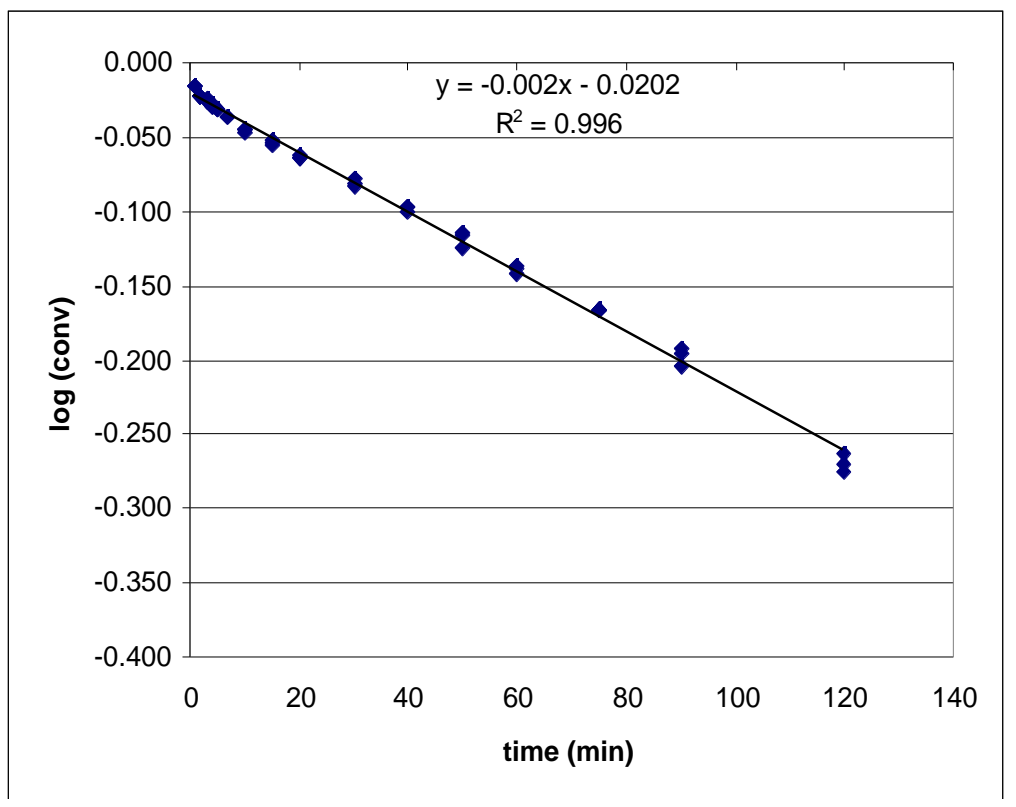




\begin{tabular}{|c|c|c|c|c|}
\hline time & area $\mathrm{A}$ & area 16 & conversion & $\log (1-$ conv $)$ \\
\hline 1 & 9251 & 446 & 0.040 & -0.018 \\
\hline 1 & 10258 & 439 & 0.036 & -0.016 \\
\hline 1 & 10028 & 436 & 0.036 & -0.016 \\
\hline 2 & 11177 & 662 & 0.049 & -0.022 \\
\hline 2 & 13484 & 797 & 0.049 & -0.022 \\
\hline 2 & 20067 & 1200 & 0.050 & -0.022 \\
\hline 3 & 9879 & 655 & 0.055 & -0.025 \\
\hline 3 & 11183 & 782 & 0.058 & -0.026 \\
\hline 3 & 12781 & 870 & 0.057 & -0.025 \\
\hline 4 & 7377 & 575 & 0.065 & -0.029 \\
\hline 4 & 8519 & 636 & 0.062 & -0.028 \\
\hline 4 & 11569 & 924 & 0.067 & -0.030 \\
\hline 5 & 12632 & 1063 & 0.070 & -0.032 \\
\hline 5 & 20536 & 1751 & 0.071 & -0.032 \\
\hline 7 & 6122 & 590 & 0.080 & -0.036 \\
\hline 7 & 7203 & 697 & 0.081 & -0.037 \\
\hline 10 & 5312 & 626 & 0.098 & -0.045 \\
\hline 10 & 5415 & 663 & 0.102 & -0.047 \\
\hline 10 & 6451 & 765 & 0.099 & -0.045 \\
\hline 15 & 5357 & 773 & 0.120 & -0.056 \\
\hline 15 & 6652 & 911 & 0.114 & -0.053 \\
\hline 15 & 6818 & 955 & 0.117 & -0.054 \\
\hline 20 & 7195 & 1195 & 0.138 & -0.065 \\
\hline 20 & 9082 & 1508 & 0.138 & -0.065 \\
\hline 20 & 8826 & 1425 & 0.135 & -0.063 \\
\hline 30 & 5584 & 1178 & 0.176 & -0.084 \\
\hline 30 & 7562 & 1557 & 0.172 & -0.082 \\
\hline 30 & 7508 & 1490 & 0.165 & -0.079 \\
\hline 40 & 5506 & 1356 & 0.205 & -0.100 \\
\hline 40 & 7023 & 1682 & 0.200 & -0.097 \\
\hline 40 & 7857 & 1898 & 0.201 & -0.098 \\
\hline 50 & 5995 & 1685 & 0.234 & -0.116 \\
\hline 50 & 6909 & 1910 & 0.230 & -0.114 \\
\hline 50 & 8348 & 2490 & 0.249 & -0.124 \\
\hline 60 & 6086 & 2032 & 0.278 & -0.142 \\
\hline 60 & 7588 & 2455 & 0.270 & -0.136 \\
\hline 60 & 8098 & 2643 & 0.272 & -0.138 \\
\hline 75 & 5592 & 2140 & 0.319 & -0.167 \\
\hline 75 & 6191 & 2360 & 0.318 & -0.166 \\
\hline 75 & 6483 & 2464 & 0.317 & -0.165 \\
\hline 90 & 5685 & 2434 & 0.357 & -0.192 \\
\hline 90 & 6820 & 3069 & 0.375 & -0.204 \\
\hline 90 & 7047 & 3067 & 0.363 & -0.196 \\
\hline 120 & 6244 & 3401 & 0.454 & -0.263 \\
\hline 120 & 7345 & 4141 & 0.470 & -0.276 \\
\hline 120 & 7167 & 3981 & 0.463 & -0.270 \\
\hline
\end{tabular}




\section{Order in Palladium, (0.05 equiv).}

(E)-1

2-Iodothiophene

Tetra- $n$-butylammonium fluoride

Bis(dibenzylideneacetone)palladium $2.5 \mathrm{mM}$ (0.05 equiv)
$50 \mathrm{mM}$ (1.0 equiv)

$50 \mathrm{mM}$ (1.0 equiv)

$188 \mathrm{mM}$ (3.75 equiv)

Following the General Procedure, silanol (E)-1 (43 mg, $0.25 \mathrm{mmol})$ and biphenyl (10.5 $\mathrm{mg}$ ) were dissolved in TBAF solution $(940 \mu \mathrm{L}, 1.0 \mathrm{M}$ in THF, $0.94 \mathrm{mmol})$ in a flame dried 2neck round-bottomed flask, equipped with a stir bar and a septum under argon. Dry THF (3.05 $\mathrm{mL}$ ) was added and the clear, slightly-yellow solution was immersed into a water-bath at $23{ }^{\circ} \mathrm{C} \pm$ $1{ }^{\circ} \mathrm{C}$ for $30 \mathrm{~min}$. The 2-iodothiophene $(28 \mu \mathrm{L}, 0.25 \mathrm{mmol})$ was then added and the reaction was stirred for $1 \mathrm{~min}$. A suspension of $\operatorname{Pd}(\mathrm{dba})_{2}(7.2 \mathrm{mg}, 0.0125 \mathrm{mmol})$ in dry THF $(1.0 \mathrm{~mL})$ was added. The initially purple, turbid reaction mixture turns into a clear solution within 30 seconds. Aliquots $(\sim 200 \mu \mathrm{L})$ were withdrawn after 1, 2, 3, 4, 5, 7, 10, 15, 20, 30, 40, 50, 60, 75, 90, and $120 \mathrm{~min}$. To quench, these samples were added to $100 \mu \mathrm{L}$ of a $20 \%$ aqueous solution of 2dimethylaminoethanethiol hydrochloride. The clear colorless to slightly-yellow solution was then filtered through a plug of silica gel, and the silica gel was washed with diethyl ether to give a total sample volume of $\sim 2 \mathrm{~mL}$. This sample was injected into the GC three times.

initial rate $=0.0094$

$\mathrm{k}_{\text {obs }}=0.0026$
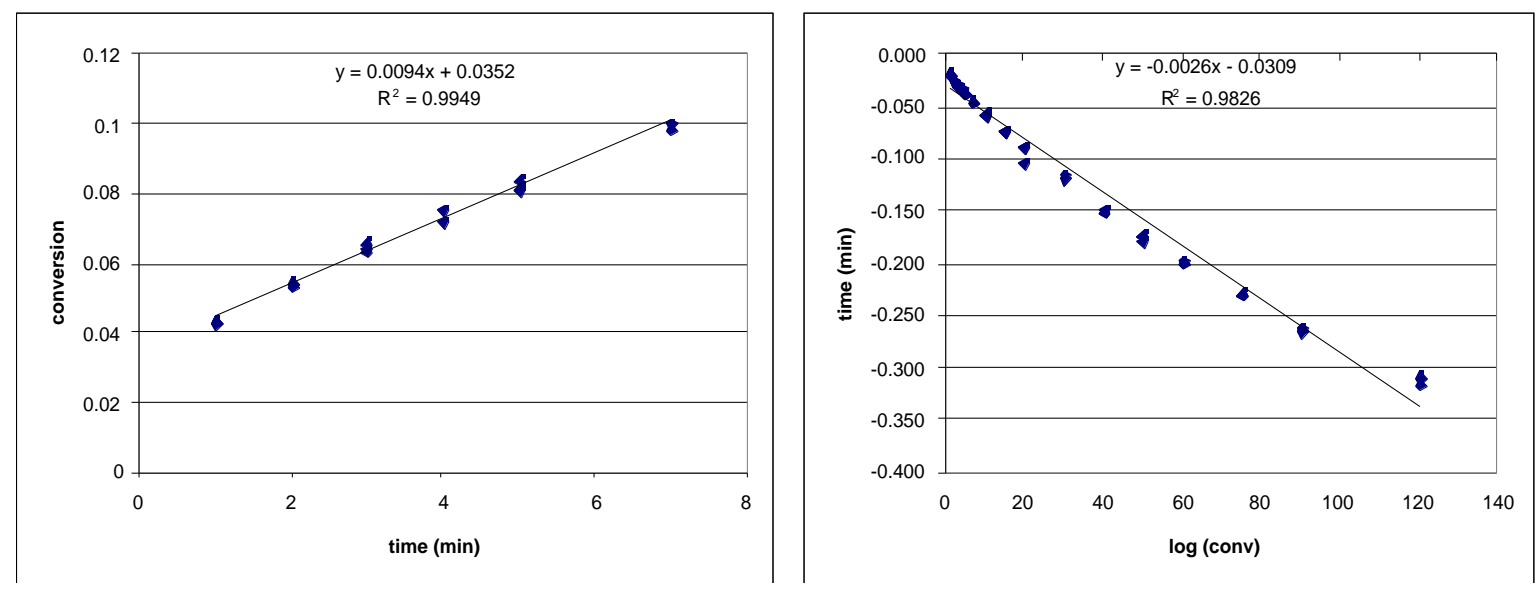


\begin{tabular}{|c|c|c|c|c|}
\hline time & area $\mathrm{A}$ & area 16 & conversion & $\log (1-\mathrm{conv})$ \\
\hline 1 & 6725 & 735 & 0.043 & -0.019 \\
\hline 1 & 7165 & 788 & 0.044 & -0.019 \\
\hline 1 & 8101 & 877 & 0.043 & -0.019 \\
\hline 2 & 5000 & 690 & 0.055 & -0.024 \\
\hline 2 & 5326 & 722 & 0.054 & -0.024 \\
\hline 2 & 5380 & 731 & 0.054 & -0.024 \\
\hline 3 & 6016 & 965 & 0.064 & -0.029 \\
\hline 3 & 6402 & 1041 & 0.064 & -0.029 \\
\hline 3 & 6682 & 1109 & 0.066 & -0.030 \\
\hline 4 & 5617 & 1073 & 0.076 & -0.034 \\
\hline 4 & 6496 & 1187 & 0.072 & -0.033 \\
\hline 5 & 8273 & 1756 & 0.084 & -0.038 \\
\hline 5 & 8892 & 1817 & 0.081 & -0.037 \\
\hline 5 & 9681 & 2002 & 0.082 & -0.037 \\
\hline 7 & 6705 & 1700 & 0.100 & -0.046 \\
\hline 7 & 7026 & 1753 & 0.099 & -0.045 \\
\hline 7 & 7151 & 1813 & 0.100 & -0.046 \\
\hline 10 & 6691 & 2104 & 0.125 & -0.058 \\
\hline 10 & 7282 & 2239 & 0.122 & -0.056 \\
\hline 10 & 7907 & 2487 & 0.125 & -0.058 \\
\hline 15 & 6182 & 2441 & 0.156 & -0.074 \\
\hline 15 & 6620 & 2585 & 0.155 & -0.073 \\
\hline 15 & 7229 & 2823 & 0.155 & -0.073 \\
\hline 20 & 7093 & 3815 & 0.213 & -0.104 \\
\hline 20 & 7695 & 3571 & 0.184 & -0.088 \\
\hline 20 & 8405 & 3904 & 0.184 & -0.088 \\
\hline 30 & 6900 & 4165 & 0.239 & -0.119 \\
\hline 30 & 7627 & 4434 & 0.230 & -0.114 \\
\hline 30 & 8816 & 5332 & 0.239 & -0.119 \\
\hline 40 & 6677 & 4881 & 0.289 & -0.148 \\
\hline 40 & 7292 & 5393 & 0.293 & -0.150 \\
\hline 40 & 8489 & 6237 & 0.291 & -0.149 \\
\hline 50 & 6209 & 5279 & 0.337 & -0.178 \\
\hline 50 & 7149 & 5918 & 0.328 & -0.173 \\
\hline 50 & 7404 & 6112 & 0.327 & -0.172 \\
\hline 60 & 7439 & 6857 & 0.365 & -0.197 \\
\hline 60 & 8409 & 7834 & 0.369 & -0.200 \\
\hline 60 & 8717 & 8078 & 0.367 & -0.199 \\
\hline 75 & 5147 & 5317 & 0.409 & -0.228 \\
\hline 75 & 5908 & 6107 & 0.409 & -0.229 \\
\hline 75 & 5766 & 5987 & 0.411 & -0.230 \\
\hline 90 & 6949 & 7942 & 0.453 & -0.262 \\
\hline 90 & 6313 & 7240 & 0.454 & -0.263 \\
\hline 90 & 7772 & 8963 & 0.457 & -0.265 \\
\hline 120 & 6152 & 8023 & 0.516 & -0.316 \\
\hline 120 & 6397 & 8256 & 0.511 & -0.311 \\
\hline 120 & 8107 & 10412 & 0.509 & -0.309 \\
\hline
\end{tabular}




\section{Order in Palladium, (0.05 equiv).}

(E)-1

2-Iodothiophene

Tetra- $n$-butylammonium fluoride

Bis(dibenzylideneacetone)palladium $2.5 \mathrm{mM}$ (0.05 equiv)
$50 \mathrm{mM}$ (1.0 equiv)

$188 \mathrm{mM}$ (3.75 equiv)
$50 \mathrm{mM}$ (1.0 equiv)

Following the General Procedure, silanol (E)-1 (43 mg, $0.25 \mathrm{mmol})$ and biphenyl (10.1 $\mathrm{mg}$ ) were dissolved in TBAF solution $(940 \mu \mathrm{L}, 1.0 \mathrm{M}$ in THF, $0.94 \mathrm{mmol})$ in a flame dried 2neck round-bottomed flask, equipped with a stir bar and a septum under argon. Dry THF (3.05 $\mathrm{mL}$ ) was added and the clear, slightly-yellow solution was immersed into a water-bath at $23{ }^{\circ} \mathrm{C} \pm$ $1{ }^{\circ} \mathrm{C}$ for $30 \mathrm{~min}$. The 2-iodothiophene $(28 \mu \mathrm{L}, 0.25 \mathrm{mmol})$ was then added and the reaction was stirred for $1 \mathrm{~min}$. A suspension of $\mathrm{Pd}(\mathrm{dba})_{2}(7.2 \mathrm{mg}, 0.0125 \mathrm{mmol})$ in dry THF $(1.0 \mathrm{~mL})$ was added. The initially purple, turbid reaction mixture turns into a clear solution within 30 seconds. Aliquots $(\sim 200 \mu \mathrm{L})$ were withdrawn after 1, 2, 3, 4, 5, 7, 10, 15, 20, 30, 40, 50, 60, 75, 90, and $120 \mathrm{~min}$. To quench, these samples were added to $100 \mu \mathrm{L}$ of a $20 \%$ aqueous solution of 2dimethylaminoethanethiol hydrochloride. The clear colorless to slightly-yellow solution was then filtered through a plug of silica gel, and the silica gel was washed with diethyl ether to give a total sample volume of $\sim 2 \mathrm{~mL}$. This sample was injected into the GC three times.

initial rate $=0.0112$

$\mathrm{k}_{\text {obs }}=0.0043$
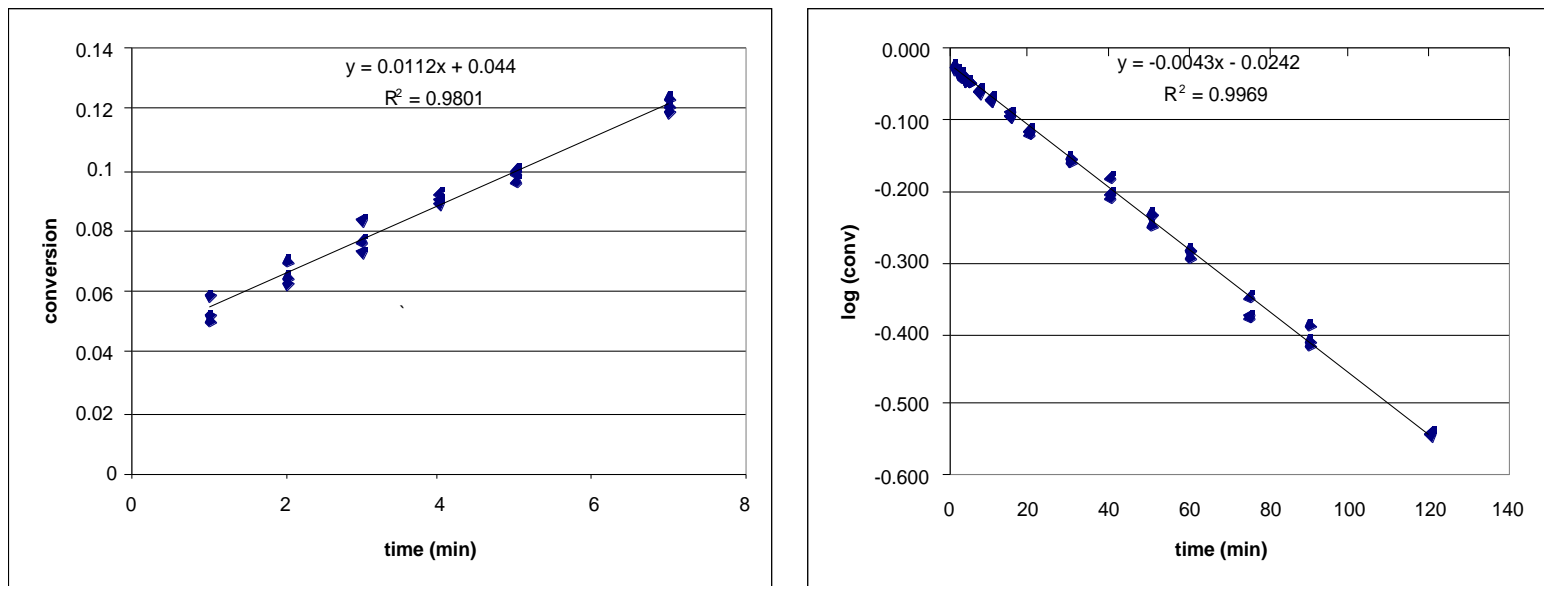


\begin{tabular}{|c|c|c|c|c|}
\hline time & area $\mathrm{A}$ & area 16 & conversion & $\log (1-\mathrm{conv})$ \\
\hline 1 & 4056 & 562 & 0.053 & -0.024 \\
\hline 1 & 4861 & 643 & 0.050 & -0.022 \\
\hline 1 & 5433 & 840 & 0.059 & -0.026 \\
\hline 2 & 4628 & 770 & 0.063 & -0.028 \\
\hline 2 & 7033 & 1200 & 0.065 & -0.029 \\
\hline 2 & 5746 & 1065 & 0.071 & -0.032 \\
\hline 3 & 4341 & 838 & 0.074 & -0.033 \\
\hline 3 & 6230 & 1263 & 0.077 & -0.035 \\
\hline 3 & 5007 & 1108 & 0.084 & -0.038 \\
\hline 4 & 3732 & 909 & 0.093 & -0.042 \\
\hline 4 & 5072 & 1205 & 0.090 & -0.041 \\
\hline 4 & 4594 & 1082 & 0.090 & -0.041 \\
\hline 5 & 4962 & 1259 & 0.097 & -0.044 \\
\hline 5 & 6751 & 1755 & 0.099 & -0.045 \\
\hline 5 & 6437 & 1702 & 0.101 & -0.046 \\
\hline 7 & 3118 & 1016 & 0.124 & -0.058 \\
\hline 7 & 4442 & 1417 & 0.122 & -0.056 \\
\hline 7 & 4348 & 1363 & 0.119 & -0.055 \\
\hline 10 & 3819 & 1478 & 0.147 & -0.069 \\
\hline 10 & 5586 & 2102 & 0.143 & -0.067 \\
\hline 10 & 5611 & 2176 & 0.148 & -0.069 \\
\hline 15 & 3477 & 1740 & 0.191 & -0.092 \\
\hline 15 & 4591 & 2189 & 0.182 & -0.087 \\
\hline 15 & 5281 & 2680 & 0.193 & -0.093 \\
\hline 20 & 4527 & 2712 & 0.228 & -0.112 \\
\hline 20 & 5840 & 3579 & 0.233 & -0.115 \\
\hline 20 & 5775 & 3630 & 0.239 & -0.119 \\
\hline 30 & 4171 & 3199 & 0.292 & -0.150 \\
\hline 30 & 5536 & 4375 & 0.301 & -0.156 \\
\hline 30 & 5632 & 4461 & 0.302 & -0.156 \\
\hline 40 & 4490 & 4014 & 0.341 & -0.181 \\
\hline 40 & 4761 & 4734 & 0.379 & -0.207 \\
\hline 40 & 4990 & 4854 & 0.371 & -0.201 \\
\hline 50 & 3884 & 4175 & 0.409 & -0.229 \\
\hline 50 & 4945 & 5382 & 0.415 & -0.233 \\
\hline 50 & 4910 & 5565 & 0.432 & -0.245 \\
\hline 60 & 4924 & 6160 & 0.477 & -0.281 \\
\hline 60 & 5922 & 7589 & 0.488 & -0.291 \\
\hline 60 & 6113 & 7703 & 0.480 & -0.284 \\
\hline 75 & 3942 & 5955 & 0.575 & -0.372 \\
\hline 75 & 4564 & 6935 & 0.579 & -0.375 \\
\hline 75 & 5161 & 7469 & 0.551 & -0.348 \\
\hline 90 & 4925 & 7884 & 0.610 & -0.409 \\
\hline 90 & 6181 & 9966 & 0.614 & -0.414 \\
\hline 90 & 5347 & 8279 & 0.590 & -0.387 \\
\hline 120 & 4737 & 8850 & 0.712 & -0.540 \\
\hline 120 & 6315 & 11773 & 0.710 & -0.538 \\
\hline
\end{tabular}




\section{Order in Palladium, (0.05 equiv).}

(E)-1

2-Iodothiophene

Tetra- $n$-butylammonium fluoride

Bis(dibenzylideneacetone)palladium $2.5 \mathrm{mM}$ (0.05 equiv)
$50 \mathrm{mM}$ (1.0 equiv)

$50 \mathrm{mM}$ (1.0 equiv)

$188 \mathrm{mM}$ (3.75 equiv)

Following the General Procedure, silanol (E)-1 (43 mg, $0.25 \mathrm{mmol})$ and biphenyl (5.8 $\mathrm{mg}$ ) were dissolved in TBAF solution $(940 \mu \mathrm{L}, 1.0 \mathrm{M}$ in THF, $0.94 \mathrm{mmol})$ in a flame dried 2neck round-bottomed flask, equipped with a stir bar and a septum under argon. Dry THF (3.05 $\mathrm{mL}$ ) was added and the clear, slightly-yellow solution was immersed into a water-bath at $23{ }^{\circ} \mathrm{C} \pm$ $1{ }^{\circ} \mathrm{C}$ for $30 \mathrm{~min}$. The 2-iodothiophene $(28 \mu \mathrm{L}, 0.25 \mathrm{mmol})$ was then added and the reaction was stirred for $1 \mathrm{~min}$. A suspension of $\operatorname{Pd}(\mathrm{dba})_{2}(7.2 \mathrm{mg}, 0.0125 \mathrm{mmol})$ in dry THF $(1.0 \mathrm{~mL})$ was added. The initially purple, turbid reaction mixture turns into a clear solution within 30 seconds. Aliquots $(\sim 200 \mu \mathrm{L})$ were withdrawn after 1, 2, 3, 4, 5, 7, 10, 15, 20, 30, 40, 50, 60, 75, 90, and $120 \mathrm{~min}$. To quench, these samples were added to $100 \mu \mathrm{L}$ of a $20 \%$ aqueous solution of 2dimethylaminoethanethiol hydrochloride. The clear colorless to slightly-yellow solution was then filtered through a plug of silica gel, and the silica gel was washed with diethyl ether to give a total sample volume of $\sim 2 \mathrm{~mL}$. This sample was injected into the GC three times.

initial rate $=0.0104$

$\mathrm{k}_{\text {obs }}=0.0029$
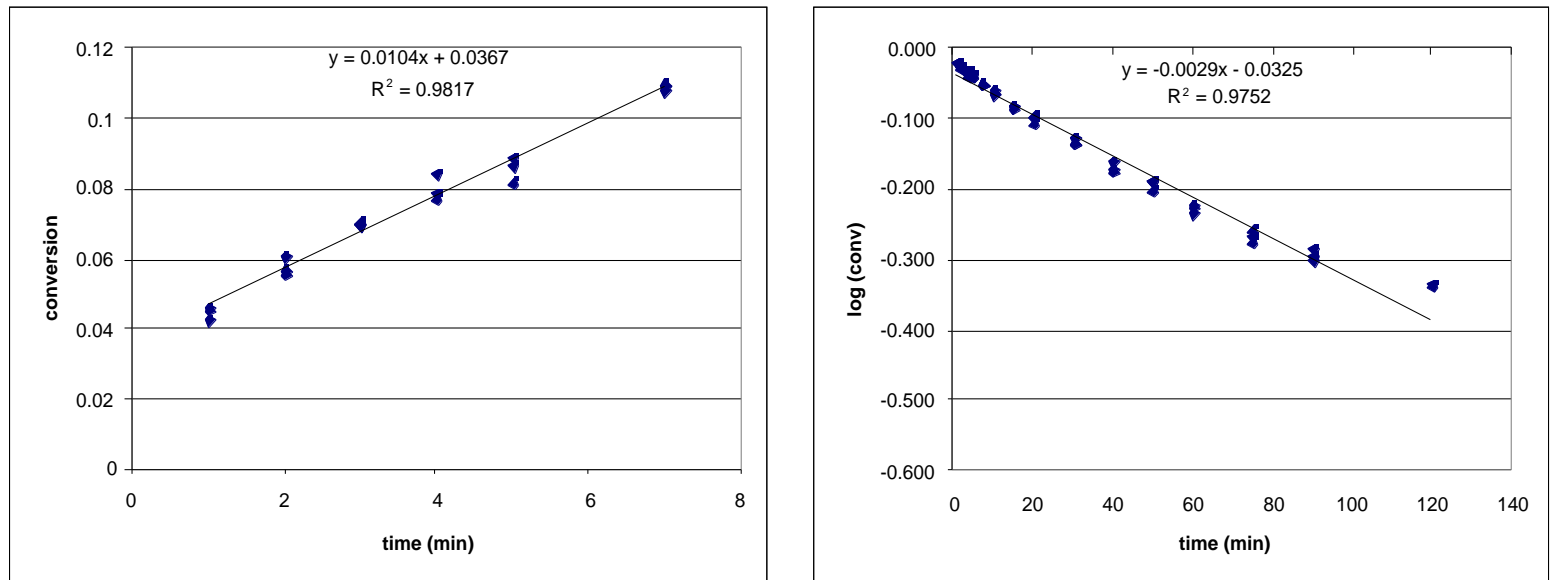


\begin{tabular}{|c|c|c|c|c|}
\hline time & area $\mathrm{A}$ & area $\mathbf{1 6}$ & conversion & $\log (1-\mathrm{conv})$ \\
\hline 1 & 3686 & 726 & 0.043 & -0.019 \\
\hline 1 & 3248 & 687 & 0.046 & -0.021 \\
\hline 1 & 3401 & 706 & 0.045 & -0.020 \\
\hline 2 & 3987 & 1037 & 0.057 & -0.025 \\
\hline 2 & 3608 & 920 & 0.056 & -0.025 \\
\hline 2 & 4021 & 1119 & 0.061 & -0.027 \\
\hline 3 & 4095 & 1315 & 0.070 & -0.032 \\
\hline 3 & 3709 & 1188 & 0.070 & -0.032 \\
\hline 3 & 4422 & 1411 & 0.070 & -0.031 \\
\hline 4 & 3620 & 1282 & 0.077 & -0.035 \\
\hline 4 & 3298 & 1275 & 0.085 & -0.038 \\
\hline 4 & 2904 & 1048 & 0.079 & -0.036 \\
\hline 5 & 3637 & 1484 & 0.089 & -0.041 \\
\hline 5 & 4109 & 1625 & 0.087 & -0.039 \\
\hline 5 & 3982 & 1492 & 0.082 & -0.037 \\
\hline 7 & 2962 & 1467 & 0.108 & -0.050 \\
\hline 7 & 3233 & 1621 & 0.110 & -0.050 \\
\hline 7 & 3603 & 1799 & 0.109 & -0.050 \\
\hline 10 & 3502 & 2047 & 0.128 & -0.059 \\
\hline 10 & 3750 & 2248 & 0.131 & -0.061 \\
\hline 10 & 3741 & 2316 & 0.135 & -0.063 \\
\hline 15 & 3841 & 2990 & 0.170 & -0.081 \\
\hline 15 & 4491 & 3460 & 0.169 & -0.080 \\
\hline 15 & 4500 & 3623 & 0.176 & -0.084 \\
\hline 20 & 3739 & 3419 & 0.200 & -0.097 \\
\hline 20 & 4147 & 3716 & 0.196 & -0.095 \\
\hline 20 & 4050 & 4042 & 0.218 & -0.107 \\
\hline 30 & 3330 & 4077 & 0.268 & -0.135 \\
\hline 30 & 3460 & 4004 & 0.253 & -0.127 \\
\hline 30 & 3554 & 4320 & 0.266 & -0.134 \\
\hline 40 & 3838 & 5813 & 0.331 & -0.175 \\
\hline 40 & 4273 & 6049 & 0.310 & -0.161 \\
\hline 40 & 4089 & 6070 & 0.325 & -0.171 \\
\hline 50 & 3572 & 5762 & 0.353 & -0.189 \\
\hline 50 & 4249 & 6847 & 0.352 & -0.189 \\
\hline 50 & 4372 & 7418 & 0.371 & -0.201 \\
\hline 60 & 4219 & 7782 & 0.403 & -0.224 \\
\hline 60 & 4489 & 8172 & 0.398 & -0.221 \\
\hline 60 & 4451 & 8471 & 0.416 & -0.234 \\
\hline 75 & 4109 & 8799 & 0.468 & -0.274 \\
\hline 75 & 4861 & 9962 & 0.448 & -0.258 \\
\hline 75 & 5558 & 11676 & 0.460 & -0.267 \\
\hline 90 & 5253 & 11809 & 0.492 & -0.294 \\
\hline 90 & 5764 & 12647 & 0.480 & -0.284 \\
\hline 90 & 5986 & 13694 & 0.500 & -0.301 \\
\hline 120 & 4130 & 10198 & 0.540 & -0.337 \\
\hline 120 & 4861 & 11931 & 0.537 & -0.334 \\
\hline
\end{tabular}




\section{Order in Palladium, (0.10 equiv).}

(E)-1

2-Iodothiophene

Tetra- $n$-butylammonium fluoride

Bis(dibenzylideneacetone)palladium $5 \mathrm{mM}$ (0.10 equiv)
$50 \mathrm{mM}$ (1.0 equiv)

$50 \mathrm{mM}$ (1.0 equiv)

$188 \mathrm{mM}$ (3.75 equiv)

Following the General Procedure, silanol (E)-1 (43 mg, $0.25 \mathrm{mmol})$ and biphenyl (13.5 $\mathrm{mg}$ ) were dissolved in TBAF solution $(940 \mu \mathrm{L}, 1.0 \mathrm{M}$ in THF, $0.94 \mathrm{mmol})$ in a flame dried 2neck round-bottomed flask, equipped with a stir bar and a septum under argon. Dry THF (3.0 $\mathrm{mL}$ ) was added and the clear, slightly-yellow solution was immersed into a water-bath at $23{ }^{\circ} \mathrm{C} \pm$ $1{ }^{\circ} \mathrm{C}$ for $30 \mathrm{~min}$. The 2-iodothiophene $(28 \mu \mathrm{L}, 0.25 \mathrm{mmol})$ was then added and the reaction was stirred for $1 \mathrm{~min}$. A suspension of $\operatorname{Pd}(\mathrm{dba})_{2}(14.4 \mathrm{mg}, 0.025 \mathrm{mmol})$ in dry THF $(1.0 \mathrm{~mL})$ was added. The initially purple, turbid reaction mixture turns into a clear solution within 30 seconds. Aliquots $(\sim 200 \mu \mathrm{L})$ were withdrawn after 1, 2, 3, 4, 5, 7, 10, 15, 20, 30, 40, 50, 60, 75, and 90 min. To quench, these samples were added to $100 \mu \mathrm{L}$ of a $20 \%$ aqueous solution of 2dimethylaminoethanethiol hydrochloride. The clear colorless to slightly-yellow solution was then filtered through a plug of silica gel, and the silica gel was washed with diethyl ether to give a total sample volume of $\sim 2 \mathrm{~mL}$. This sample was injected into the GC three times.

$\mathrm{k}_{\mathrm{obs}}=0.0086$

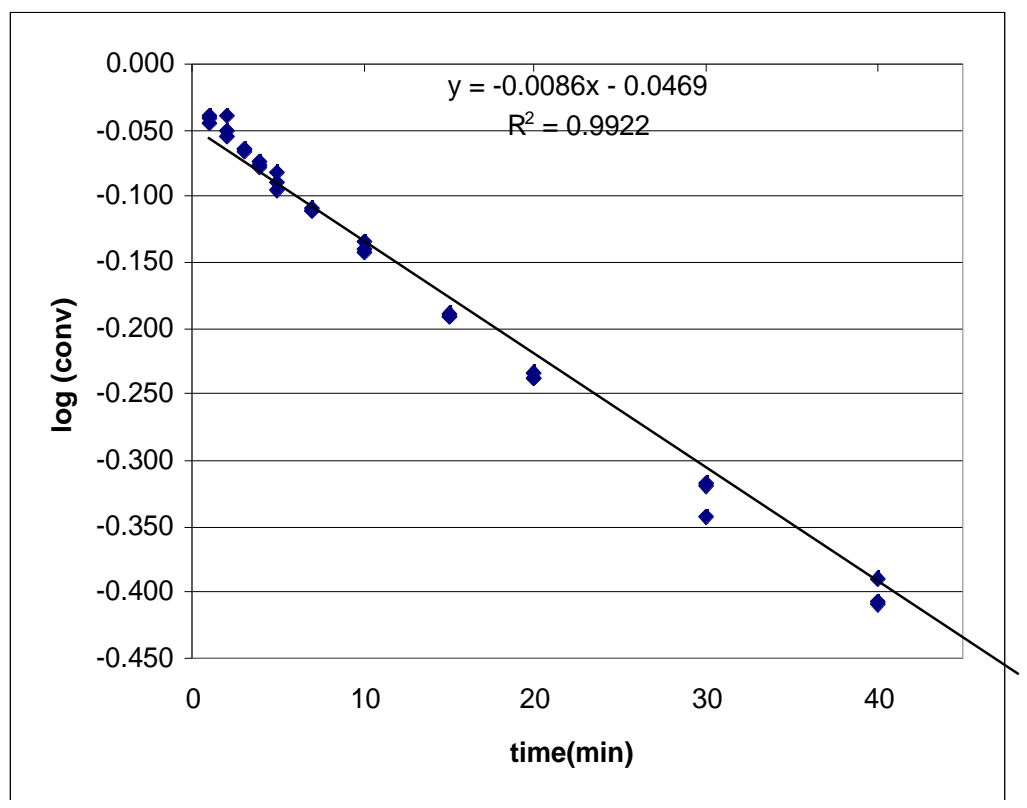




\begin{tabular}{|c|c|c|c|c|}
\hline time & $\operatorname{area} \mathrm{A}$ & area $\mathbf{1 6}$ & conversion & $\log (1-\operatorname{conv})$ \\
\hline 1 & 5469 & 1041 & 0.097 & -0.044 \\
\hline 1 & 5995 & 1025 & 0.087 & -0.040 \\
\hline 1 & 6427 & 1115 & 0.088 & -0.040 \\
\hline 2 & 6210 & 1421 & 0.116 & -0.054 \\
\hline 2 & 6928 & 1151 & 0.085 & -0.038 \\
\hline 2 & 7766 & 1702 & 0.112 & -0.051 \\
\hline 3 & 6641 & 1788 & 0.137 & -0.064 \\
\hline 3 & 7115 & 1963 & 0.140 & -0.066 \\
\hline 3 & 8262 & 2213 & 0.136 & -0.064 \\
\hline 4 & 6884 & 2215 & 0.164 & -0.078 \\
\hline 4 & 7502 & 2334 & 0.158 & -0.075 \\
\hline 4 & 9174 & 2880 & 0.160 & -0.076 \\
\hline 5 & 6001 & 2201 & 0.187 & -0.090 \\
\hline 5 & 6647 & 2575 & 0.197 & -0.095 \\
\hline 5 & 8429 & 2867 & 0.173 & -0.083 \\
\hline 7 & 5034 & 2227 & 0.225 & -0.111 \\
\hline 7 & 5178 & 2266 & 0.223 & -0.109 \\
\hline 7 & 6517 & 2867 & 0.224 & -0.110 \\
\hline 10 & 5127 & 2803 & 0.278 & -0.142 \\
\hline 10 & 5402 & 2936 & 0.277 & -0.141 \\
\hline 10 & 6837 & 3589 & 0.267 & -0.135 \\
\hline 15 & 5710 & 3993 & 0.356 & -0.191 \\
\hline 15 & 6891 & 4787 & 0.354 & -0.190 \\
\hline 15 & 7280 & 5092 & 0.356 & -0.191 \\
\hline 20 & 5277 & 4357 & 0.420 & -0.237 \\
\hline 20 & 5548 & 4594 & 0.422 & -0.238 \\
\hline 20 & 6674 & 5445 & 0.415 & -0.233 \\
\hline 30 & 5199 & 5575 & 0.546 & -0.343 \\
\hline 30 & 5559 & 5684 & 0.521 & -0.319 \\
\hline 30 & 7032 & 7165 & 0.519 & -0.318 \\
\hline 40 & 5034 & 6011 & 0.608 & -0.407 \\
\hline 40 & 4566 & 5473 & 0.610 & -0.409 \\
\hline 40 & 6717 & 7809 & 0.592 & -0.389 \\
\hline 50 & 7029 & 9102 & 0.659 & -0.468 \\
\hline 50 & 7258 & 9505 & 0.667 & -0.477 \\
\hline 50 & 9547 & 12502 & 0.667 & -0.477 \\
\hline 60 & 5156 & 7258 & 0.717 & -0.548 \\
\hline 60 & 5206 & 7363 & 0.720 & -0.553 \\
\hline 60 & 6753 & 9258 & 0.698 & -0.520 \\
\hline 75 & 5096 & 7823 & 0.782 & -0.661 \\
\hline 75 & 5383 & 8252 & 0.780 & -0.659 \\
\hline 75 & 7424 & 11185 & 0.767 & -0.633 \\
\hline 90 & 4448 & 7369 & 0.843 & -0.805 \\
\hline 90 & 5222 & 8376 & 0.817 & -0.737 \\
\hline 90 & 6222 & 9740 & 0.797 & -0.692 \\
\hline
\end{tabular}




\section{Order in Palladium, (0.10 equiv).}

(E)-1

2-Iodothiophene

Tetra- $n$-butylammonium fluoride

Bis(dibenzylideneacetone)palladium $5 \mathrm{mM}$ (0.10 equiv)
$50 \mathrm{mM}$ (1.0 equiv)

$50 \mathrm{mM}$ (1.0 equiv)

$188 \mathrm{mM}$ (3.75 equiv)

Following the General Procedure, silanol (E)-1 (43 mg, $0.25 \mathrm{mmol})$ and biphenyl (13.1 $\mathrm{mg}$ ) were dissolved in TBAF solution $(940 \mu \mathrm{L}, 1.0 \mathrm{M}$ in THF, $0.94 \mathrm{mmol})$ in a flame dried 2neck round-bottomed flask, equipped with a stir bar and a septum under argon. Dry THF (3.0 $\mathrm{mL}$ ) was added and the clear, slightly-yellow solution was immersed into a water-bath at $23{ }^{\circ} \mathrm{C} \pm$ $1{ }^{\circ} \mathrm{C}$ for $30 \mathrm{~min}$. The 2-iodothiophene $(28 \mu \mathrm{L}, 0.25 \mathrm{mmol})$ was then added and the reaction was stirred for $1 \mathrm{~min}$. A suspension of $\operatorname{Pd}(\mathrm{dba})_{2}(14.4 \mathrm{mg}, 0.025 \mathrm{mmol})$ in dry THF $(1.0 \mathrm{~mL})$ was added. The initially purple, turbid reaction mixture turns into a clear solution within 30 seconds. Aliquots $(\sim 200 \mu \mathrm{L})$ were withdrawn after 1, 2, 3, 4, 5, 7, 10, 15, 20, 30, 40, 50, 60, 75, and 90 min. To quench, these samples were added to $100 \mu \mathrm{L}$ of a $20 \%$ aqueous solution of 2dimethylaminoethanethiol hydrochloride. The clear colorless to slightly-yellow solution was then filtered through a plug of silica gel, and the silica gel was washed with diethyl ether to give a total sample volume of $\sim 2 \mathrm{~mL}$. This sample was injected into the GC three times.

$\mathrm{k}_{\mathrm{obs}}=0.00077$

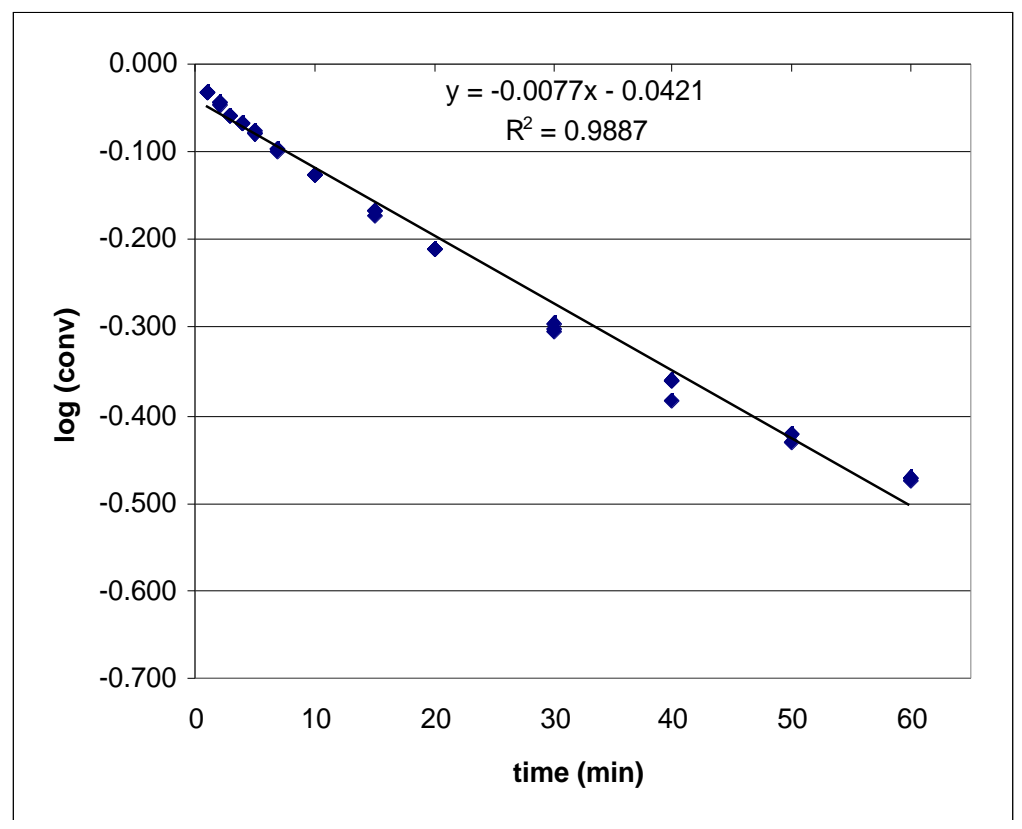




\begin{tabular}{|c|c|c|c|c|}
\hline time & $\operatorname{area} \mathrm{A}$ & area $\mathbf{1 6}$ & conversion & $\log (1-\mathrm{conv})$ \\
\hline 1 & 10778 & 1528 & 0.070 & -0.032 \\
\hline 1 & 12205 & 1754 & 0.071 & -0.032 \\
\hline 1 & 14419 & 2107 & 0.072 & -0.033 \\
\hline 2 & 12816 & 2495 & 0.096 & -0.044 \\
\hline 2 & 13795 & 2834 & 0.101 & -0.046 \\
\hline 2 & 17491 & 3490 & 0.099 & -0.045 \\
\hline 3 & 12008 & 3046 & 0.125 & -0.058 \\
\hline 3 & 12423 & 3121 & 0.124 & -0.058 \\
\hline 3 & 16069 & 4050 & 0.125 & -0.058 \\
\hline 4 & 12222 & 3520 & 0.142 & -0.067 \\
\hline 4 & 12946 & 3756 & 0.143 & -0.067 \\
\hline 4 & 16895 & 4932 & 0.144 & -0.068 \\
\hline 5 & 13574 & 4522 & 0.165 & -0.078 \\
\hline 5 & 14410 & 4743 & 0.163 & -0.077 \\
\hline 5 & 19362 & 6479 & 0.165 & -0.078 \\
\hline 7 & 13470 & 5470 & 0.201 & -0.097 \\
\hline 7 & 14429 & 5860 & 0.201 & -0.097 \\
\hline 7 & 17736 & 7262 & 0.202 & -0.098 \\
\hline 10 & 6259 & 3164 & 0.250 & -0.125 \\
\hline 10 & 6604 & 3335 & 0.249 & -0.125 \\
\hline 10 & 8295 & 4219 & 0.251 & -0.126 \\
\hline 15 & 5540 & 3591 & 0.320 & -0.168 \\
\hline 15 & 5888 & 3809 & 0.320 & -0.167 \\
\hline 15 & 7599 & 5029 & 0.327 & -0.172 \\
\hline 20 & 6660 & 5182 & 0.384 & -0.211 \\
\hline 20 & 7026 & 5464 & 0.384 & -0.211 \\
\hline 20 & 9274 & 7240 & 0.386 & -0.212 \\
\hline 30 & 7013 & 7088 & 0.499 & -0.300 \\
\hline 30 & 9316 & 9475 & 0.502 & -0.303 \\
\hline 30 & 10667 & 10675 & 0.494 & -0.296 \\
\hline 40 & 7169 & 8195 & 0.565 & -0.361 \\
\hline 40 & 7851 & 9312 & 0.586 & -0.383 \\
\hline 40 & 10573 & 12090 & 0.565 & -0.361 \\
\hline 50 & 7303 & 9198 & 0.622 & -0.423 \\
\hline 50 & 8210 & 10472 & 0.630 & -0.432 \\
\hline 50 & 11081 & 13922 & 0.621 & -0.421 \\
\hline 60 & 7364 & 9891 & 0.664 & -0.473 \\
\hline 60 & 8425 & 11293 & 0.662 & -0.471 \\
\hline 60 & 10302 & 13804 & 0.662 & -0.471 \\
\hline 75 & 7691 & 11150 & 0.716 & -0.547 \\
\hline 75 & 10406 & 14816 & 0.703 & -0.528 \\
\hline 75 & 13160 & 18810 & 0.706 & -0.532 \\
\hline 90 & 7620 & 10810 & 0.701 & -0.524 \\
\hline 90 & 9711 & 14533 & 0.739 & -0.584 \\
\hline 90 & 12370 & 18454 & 0.737 & -0.580 \\
\hline
\end{tabular}




\section{Order in Palladium, (0.10 equiv).}

(E)-1

2-Iodothiophene

Tetra- $n$-butylammonium fluoride

Bis(dibenzylideneacetone)palladium $5 \mathrm{mM}$ (0.10 equiv)
$50 \mathrm{mM}$ (1.0 equiv)

$50 \mathrm{mM}$ (1.0 equiv)

$188 \mathrm{mM}$ (3.75 equiv)

Following the General Procedure, silanol (E)-1 (43 mg, $0.25 \mathrm{mmol})$ and biphenyl (8.4 $\mathrm{mg}$ ) were dissolved in TBAF solution $(940 \mu \mathrm{L}, 1.0 \mathrm{M}$ in THF, $0.94 \mathrm{mmol})$ in a flame dried 2neck round-bottomed flask, equipped with a stir bar and a septum under argon. Dry THF (3.0 $\mathrm{mL}$ ) was added and the clear, slightly-yellow solution was immersed into a water-bath at $23{ }^{\circ} \mathrm{C} \pm$ $1{ }^{\circ} \mathrm{C}$ for $30 \mathrm{~min}$. The 2-iodothiophene $(28 \mu \mathrm{L}, 0.25 \mathrm{mmol})$ was then added and the reaction was stirred for $1 \mathrm{~min}$. A suspension of $\operatorname{Pd}(\mathrm{dba})_{2}(14.4 \mathrm{mg}, 0.025 \mathrm{mmol})$ in dry THF $(1.0 \mathrm{~mL})$ was added. The initially purple, turbid reaction mixture turns into a clear solution within 30 seconds. Aliquots $(\sim 200 \mu \mathrm{L})$ were withdrawn after 1, 2, 3, 4, 5, 7, 10, 15, 20, 30, 40, 50, 60, 75, 90, and $120 \mathrm{~min}$. To quench, these samples were added to $100 \mu \mathrm{L}$ of a $20 \%$ aqueous solution of 2dimethylaminoethanethiol hydrochloride. The clear colorless to slightly-yellow solution was then filtered through a plug of silica gel, and the silica gel was washed with diethyl ether to give a total sample volume of $\sim 2 \mathrm{~mL}$. This sample was injected into the GC three times.

$\mathrm{k}_{\mathrm{obs}}=0.0068$

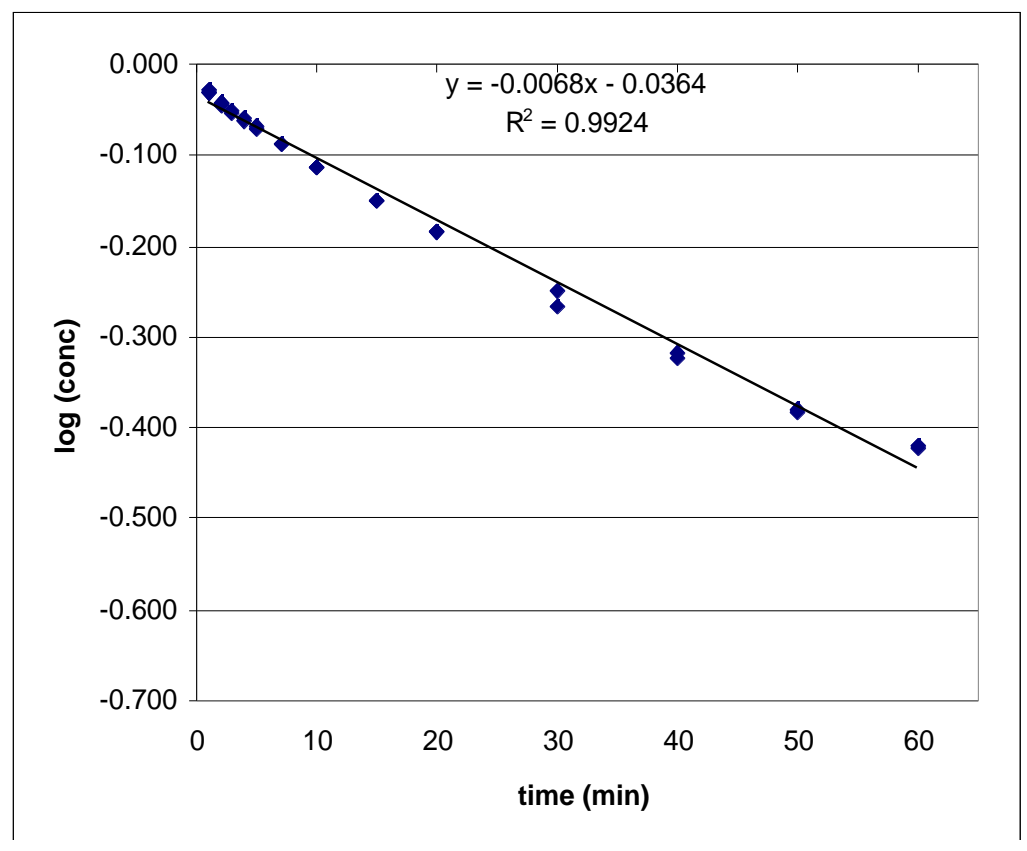




\begin{tabular}{|c|c|c|c|c|}
\hline time & area $\mathrm{A}$ & area 16 & conversion & $\log (1-$ conv $)$ \\
\hline 1 & 5699 & 1164 & 0.065 & -0.029 \\
\hline 1 & 5228 & 1096 & 0.066 & -0.030 \\
\hline 1 & 4426 & 937 & 0.067 & -0.030 \\
\hline 2 & 4072 & 1242 & 0.097 & -0.044 \\
\hline 2 & 5247 & 1543 & 0.093 & -0.042 \\
\hline 2 & 4397 & 1282 & 0.092 & -0.042 \\
\hline 3 & 4812 & 1756 & 0.116 & -0.053 \\
\hline 3 & 4495 & 1600 & 0.113 & -0.052 \\
\hline 4 & 4661 & 1872 & 0.127 & -0.059 \\
\hline 4 & 4138 & 1720 & 0.132 & -0.061 \\
\hline 5 & 5521 & 2634 & 0.151 & -0.071 \\
\hline 5 & 4445 & 2060 & 0.147 & -0.069 \\
\hline 7 & 4343 & 2545 & 0.186 & -0.089 \\
\hline 7 & 3792 & 2170 & 0.181 & -0.087 \\
\hline 10 & 4861 & 3527 & 0.230 & -0.113 \\
\hline 10 & 4878 & 3536 & 0.230 & -0.113 \\
\hline 15 & 4942 & 4557 & 0.292 & -0.150 \\
\hline 15 & 3945 & 3624 & 0.291 & -0.149 \\
\hline 20 & 5215 & 5686 & 0.345 & -0.184 \\
\hline 20 & 4397 & 4797 & 0.346 & -0.184 \\
\hline 30 & 5126 & 7057 & 0.436 & -0.249 \\
\hline 30 & 4571 & 6609 & 0.458 & -0.266 \\
\hline 40 & 5529 & 9043 & 0.518 & -0.317 \\
\hline 40 & 4684 & 7740 & 0.523 & -0.322 \\
\hline 50 & 4553 & 8389 & 0.584 & -0.381 \\
\hline 50 & 4499 & 8329 & 0.586 & -0.383 \\
\hline 60 & 5157 & 10098 & 0.620 & -0.421 \\
\hline 60 & 4144 & 8129 & 0.621 & -0.422 \\
\hline 75 & 6350 & 13495 & 0.673 & -0.486 \\
\hline 75 & 4860 & 10413 & 0.679 & -0.493 \\
\hline 90 & 6454 & 14407 & 0.707 & -0.533 \\
\hline 90 & 4684 & 10480 & 0.709 & -0.536 \\
\hline 120 & 6233 & 14746 & 0.749 & -0.601 \\
\hline 120 & 4905 & 11683 & 0.755 & -0.610 \\
\hline
\end{tabular}




\section{Order in (E)-1, (0.5 equiv).}

(E)-1

2-Iodothiophene

Tetra- $n$-butylammonium fluoride

Bis(dibenzylideneacetone)palladium $2.5 \mathrm{mM}$ (0.05 equiv)
$25 \mathrm{mM}$ ( 0.5 equiv $)$

$50 \mathrm{mM}$ (1.0 equiv)

$188 \mathrm{mM}$ (3.75 equiv)

Following the General Procedure, silanol $(E)-1(22 \mathrm{mg}, 0.125 \mathrm{mmol})$ and biphenyl $(3.8$ $\mathrm{mg}$ ) were dissolved in TBAF solution $(940 \mu \mathrm{L}, 1.0 \mathrm{M}$ in THF, $0.94 \mathrm{mmol})$ in a flame dried 2neck round-bottomed flask, equipped with a stir bar and a septum under argon. Dry THF (3.05 $\mathrm{mL}$ ) was added and the clear, slightly-yellow solution was immersed into a water-bath at $23{ }^{\circ} \mathrm{C} \pm$ $1{ }^{\circ} \mathrm{C}$ for $30 \mathrm{~min}$. The 2-iodothiophene $(28 \mu \mathrm{L}, 0.25 \mathrm{mmol})$ was then added and the reaction was stirred for $1 \mathrm{~min}$. A suspension of $\operatorname{Pd}(\mathrm{dba})_{2}(7.2 \mathrm{mg}, 0.0125 \mathrm{mmol})$ in dry THF $(1.0 \mathrm{~mL})$ was added. The initially purple, turbid reaction mixture turns into a clear solution within 30 seconds. Aliquots $(\sim 200 \mu \mathrm{L})$ were withdrawn after 1, 2, 3, 4, 5, 7, 10, 15, 20, 30, 40, 50, 60, 75, 90, and $120 \mathrm{~min}$. To quench, these samples were added to $100 \mu \mathrm{L}$ of a $20 \%$ aqueous solution of 2dimethylaminoethanethiol hydrochloride. The clear colorless to slightly-yellow solution was then filtered through a plug of silica gel, and the silica gel was washed with diethyl ether to give a total sample volume of $\sim 2 \mathrm{~mL}$. This sample was injected into the GC three times.

initial rate $=0.0024$

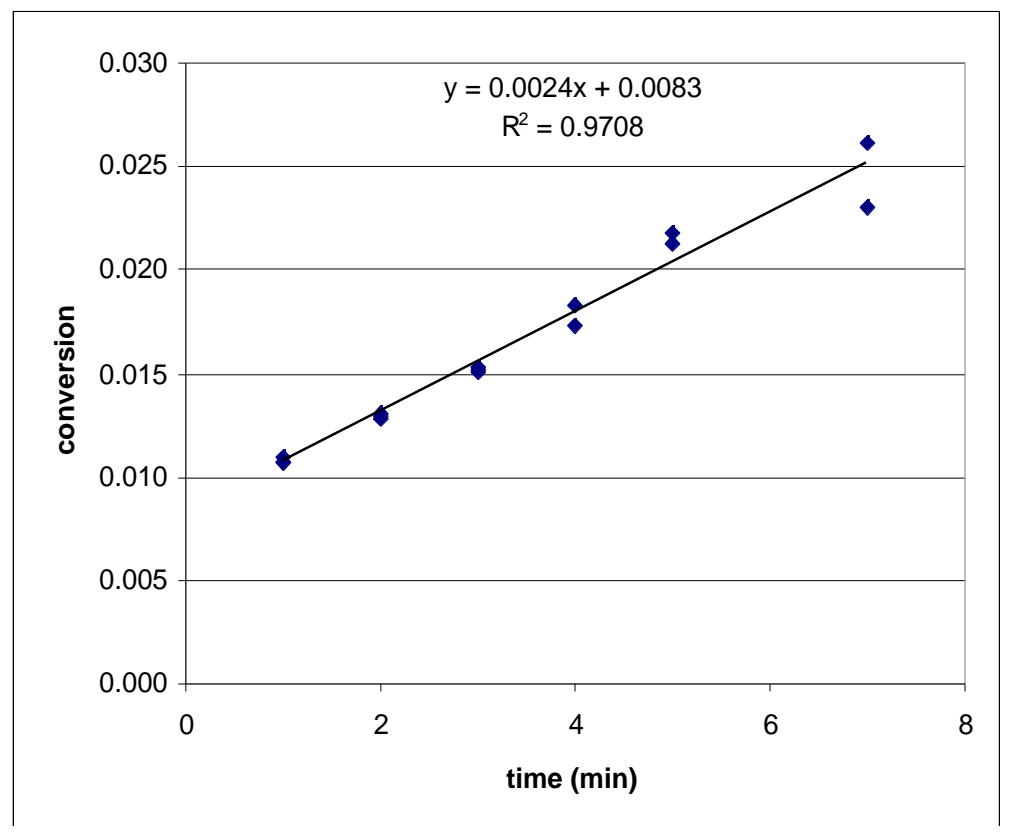




\begin{tabular}{|c|c|c|c|}
\hline time & area $\mathrm{A}$ & area 16 & conversion \\
\hline 1 & 5182 & 389 & 0.011 \\
\hline 1 & 6300 & 473 & 0.011 \\
\hline 1 & 7149 & 545 & 0.011 \\
\hline 2 & 5770 & 528 & 0.013 \\
\hline 2 & 6427 & 573 & 0.013 \\
\hline 2 & 7161 & 645 & 0.013 \\
\hline 3 & 3438 & 367 & 0.015 \\
\hline 3 & 3649 & 387 & 0.015 \\
\hline 3 & 4261 & 448 & 0.015 \\
\hline 4 & 4752 & 607 & 0.018 \\
\hline 4 & 6116 & 741 & 0.017 \\
\hline 5 & 4401 & 669 & 0.022 \\
\hline 5 & 4560 & 677 & 0.021 \\
\hline 7 & 2912 & 530 & 0.026 \\
\hline 7 & 3561 & 571 & 0.023 \\
\hline 10 & 3943 & 790 & 0.029 \\
\hline 10 & 4057 & 796 & 0.028 \\
\hline 10 & 5061 & 973 & 0.028 \\
\hline 15 & 4448 & 1117 & 0.036 \\
\hline 15 & 4992 & 1126 & 0.032 \\
\hline 15 & 5772 & 1327 & 0.033 \\
\hline 20 & 3263 & 883 & 0.039 \\
\hline 20 & 3521 & 954 & 0.039 \\
\hline 20 & 3864 & 927 & 0.034 \\
\hline 30 & 3896 & 1343 & 0.049 \\
\hline 30 & 4513 & 1460 & 0.046 \\
\hline 30 & 4724 & 1540 & 0.047 \\
\hline 40 & 5354 & 2010 & 0.054 \\
\hline 40 & 6322 & 2380 & 0.054 \\
\hline 40 & 6804 & 2616 & 0.055 \\
\hline 50 & 3257 & 1422 & 0.063 \\
\hline 50 & 3408 & 1460 & 0.061 \\
\hline 50 & 3857 & 1706 & 0.063 \\
\hline 60 & 5105 & 2439 & 0.068 \\
\hline 60 & 5743 & 2784 & 0.069 \\
\hline 60 & 7034 & 3331 & 0.068 \\
\hline 75 & 4249 & 2212 & 0.075 \\
\hline 75 & 4477 & 2346 & 0.075 \\
\hline 75 & 5418 & 2869 & 0.076 \\
\hline 90 & 4686 & 2714 & 0.083 \\
\hline 90 & 4947 & 2934 & 0.085 \\
\hline 90 & 5934 & 3496 & 0.084 \\
\hline 120 & 4771 & 3287 & 0.099 \\
\hline 120 & 4745 & 3316 & 0.100 \\
\hline 120 & 6201 & 4257 & 0.098 \\
\hline
\end{tabular}




\section{Order in (E)-1, (0.5 equiv).}

(E)-1

2-Iodothiophene

Tetra- $n$-butylammonium fluoride

Bis(dibenzylideneacetone)palladium $2.5 \mathrm{mM}$ (0.05 equiv)
$25 \mathrm{mM}$ ( 0.5 equiv $)$

$50 \mathrm{mM}$ (1.0 equiv)

$188 \mathrm{mM}$ (3.75 equiv)

Following the General Procedure, silanol $(E)-1(22 \mathrm{mg}, 0.125 \mathrm{mmol})$ and biphenyl $(3.5$ $\mathrm{mg}$ ) were dissolved in TBAF solution $(940 \mu \mathrm{L}, 1.0 \mathrm{M}$ in THF, $0.94 \mathrm{mmol})$ in a flame dried 2neck round-bottomed flask, equipped with a stir bar and a septum under argon. Dry THF (3.05 $\mathrm{mL}$ ) was added and the clear, slightly-yellow solution was immersed into a water-bath at $23{ }^{\circ} \mathrm{C} \pm$ $1{ }^{\circ} \mathrm{C}$ for $30 \mathrm{~min}$. The 2-iodothiophene $(28 \mu \mathrm{L}, 0.25 \mathrm{mmol})$ was then added and the reaction was stirred for $1 \mathrm{~min}$. A suspension of $\operatorname{Pd}(\mathrm{dba})_{2}(7.2 \mathrm{mg}, 0.0125 \mathrm{mmol})$ in dry THF $(1.0 \mathrm{~mL})$ was added. The initially purple, turbid reaction mixture turns into a clear solution within 30 seconds. Aliquots $(\sim 200 \mu \mathrm{L})$ were withdrawn after 1, 2, 3, 4, 5, 7, 10, 15, 20, 30, 40, 50, 60, 75, 90, and $120 \mathrm{~min}$. To quench, these samples were added to $100 \mu \mathrm{L}$ of a $20 \%$ aqueous solution of 2dimethylaminoethanethiol hydrochloride. The clear colorless to slightly-yellow solution was then filtered through a plug of silica gel, and the silica gel was washed with diethyl ether to give a total sample volume of $\sim 2 \mathrm{~mL}$. This sample was injected into the GC three times.

initial rate $=0.0021$

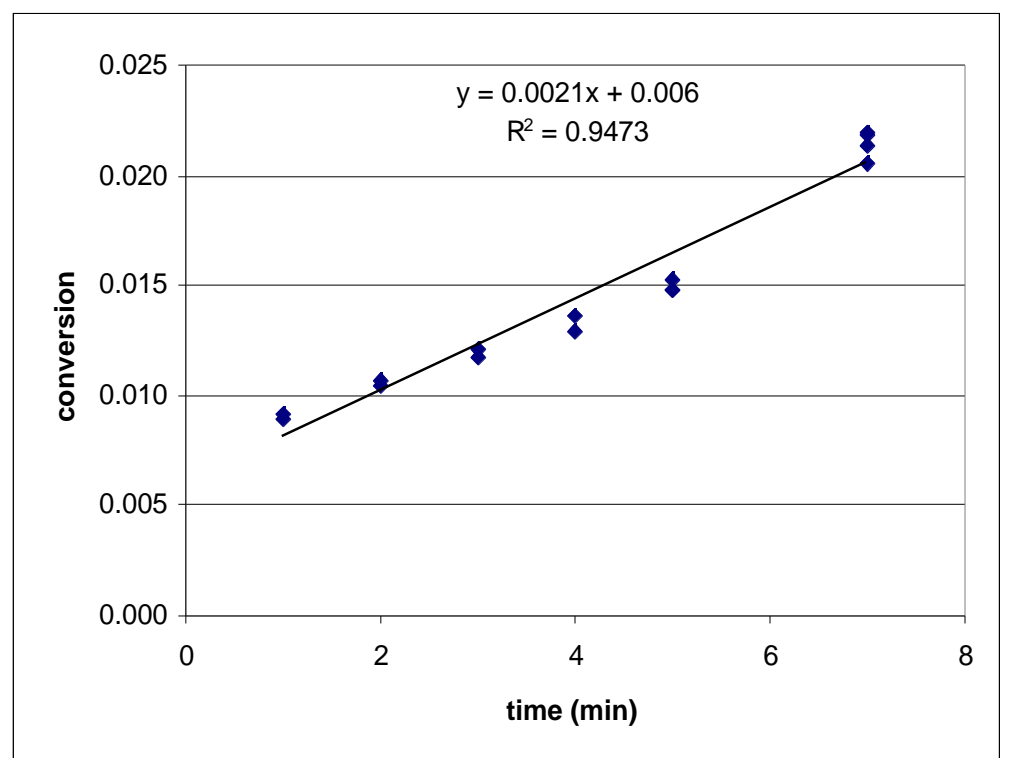




\begin{tabular}{|c|c|c|c|}
\hline time & area $\mathrm{A}$ & area 16 & conversion \\
\hline 1 & 14303 & 998 & 0.009 \\
\hline 1 & 14177 & 957 & 0.009 \\
\hline 2 & 8490 & 675 & 0.010 \\
\hline 2 & 9854 & 796 & 0.011 \\
\hline 3 & 11021 & 1011 & 0.012 \\
\hline 3 & 11718 & 1037 & 0.012 \\
\hline 4 & 6870 & 710 & 0.014 \\
\hline 4 & 8804 & 858 & 0.013 \\
\hline 5 & 6108 & 707 & 0.015 \\
\hline 5 & 7400 & 832 & 0.015 \\
\hline 5 & 7074 & 793 & 0.015 \\
\hline 7 & 3432 & 534 & 0.021 \\
\hline 7 & 8862 & 1474 & 0.022 \\
\hline 7 & 10679 & 1732 & 0.021 \\
\hline 7 & 8967 & 1487 & 0.022 \\
\hline 10 & 3174 & 471 & 0.020 \\
\hline 10 & 3740 & 552 & 0.019 \\
\hline 10 & 3889 & 530 & 0.018 \\
\hline 15 & 2139 & 413 & 0.025 \\
\hline 15 & 2683 & 558 & 0.027 \\
\hline 15 & 3153 & 664 & 0.028 \\
\hline 20 & 3503 & 563 & 0.021 \\
\hline 20 & 4108 & 956 & 0.031 \\
\hline 20 & 4473 & 1095 & 0.032 \\
\hline 30 & 4053 & 1238 & 0.040 \\
\hline 30 & 4771 & 1438 & 0.040 \\
\hline 30 & 5394 & 1503 & 0.037 \\
\hline 40 & 4978 & 1661 & 0.044 \\
\hline 40 & 6582 & 2181 & 0.044 \\
\hline 40 & 6648 & 2103 & 0.042 \\
\hline 50 & 4519 & 1794 & 0.052 \\
\hline 50 & 5699 & 2169 & 0.050 \\
\hline 50 & 6624 & 2518 & 0.050 \\
\hline 60 & 4193 & 1738 & 0.055 \\
\hline 60 & 5377 & 2300 & 0.056 \\
\hline 60 & 6840 & 2740 & 0.053 \\
\hline 75 & 4095 & 1977 & 0.064 \\
\hline 75 & 5296 & 2391 & 0.060 \\
\hline 75 & 6333 & 2905 & 0.061 \\
\hline 90 & 4861 & 2692 & 0.073 \\
\hline 90 & 6339 & 3342 & 0.070 \\
\hline 90 & 7070 & 3489 & 0.065 \\
\hline 120 & 4726 & 2949 & 0.082 \\
\hline 120 & 5740 & 3597 & 0.099 \\
\hline 120 & 7093 & 4431 & 0.099 \\
\hline
\end{tabular}




\section{Order in (E)-1, (0.5 equiv).}

(E)-1

2-Iodothiophene

Tetra- $n$-butylammonium fluoride

Bis(dibenzylideneacetone)palladium $2.5 \mathrm{mM}$ (0.05 equiv)
$25 \mathrm{mM}$ ( 0.5 equiv $)$

$50 \mathrm{mM}$ (1.0 equiv)

$188 \mathrm{mM}$ (3.75 equiv)

Following the General Procedure, silanol $(E)-1(22 \mathrm{mg}, 0.125 \mathrm{mmol})$ and biphenyl $(3.6$ $\mathrm{mg}$ ) were dissolved in TBAF solution $(940 \mu \mathrm{L}, 1.0 \mathrm{M}$ in THF, $0.94 \mathrm{mmol})$ in a flame dried 2neck round-bottomed flask, equipped with a stir bar and a septum under argon. Dry THF (3.05 $\mathrm{mL}$ ) was added and the clear, slightly-yellow solution was immersed into a water-bath at $23{ }^{\circ} \mathrm{C} \pm$ $1{ }^{\circ} \mathrm{C}$ for $30 \mathrm{~min}$. The 2-iodothiophene $(28 \mu \mathrm{L}, 0.25 \mathrm{mmol})$ was then added and the reaction was stirred for $1 \mathrm{~min}$. A suspension of $\operatorname{Pd}(\mathrm{dba})_{2}(7.2 \mathrm{mg}, 0.0125 \mathrm{mmol})$ in dry THF $(1.0 \mathrm{~mL})$ was added. The initially purple, turbid reaction mixture turns into a clear solution within 30 seconds. Aliquots $(\sim 200 \mu \mathrm{L})$ were withdrawn after 1, 2, 3, 4, 5, 7, 10, 15, 20, 30, 40, 50, 60, 75, 90, and $120 \mathrm{~min}$. To quench, these samples were added to $100 \mu \mathrm{L}$ of a $20 \%$ aqueous solution of 2dimethylaminoethanethiol hydrochloride. The clear colorless to slightly-yellow solution was then filtered through a plug of silica gel, and the silica gel was washed with diethyl ether to give a total sample volume of $\sim 2 \mathrm{~mL}$. This sample was injected into the GC three times.

initial rate $=0.0018$

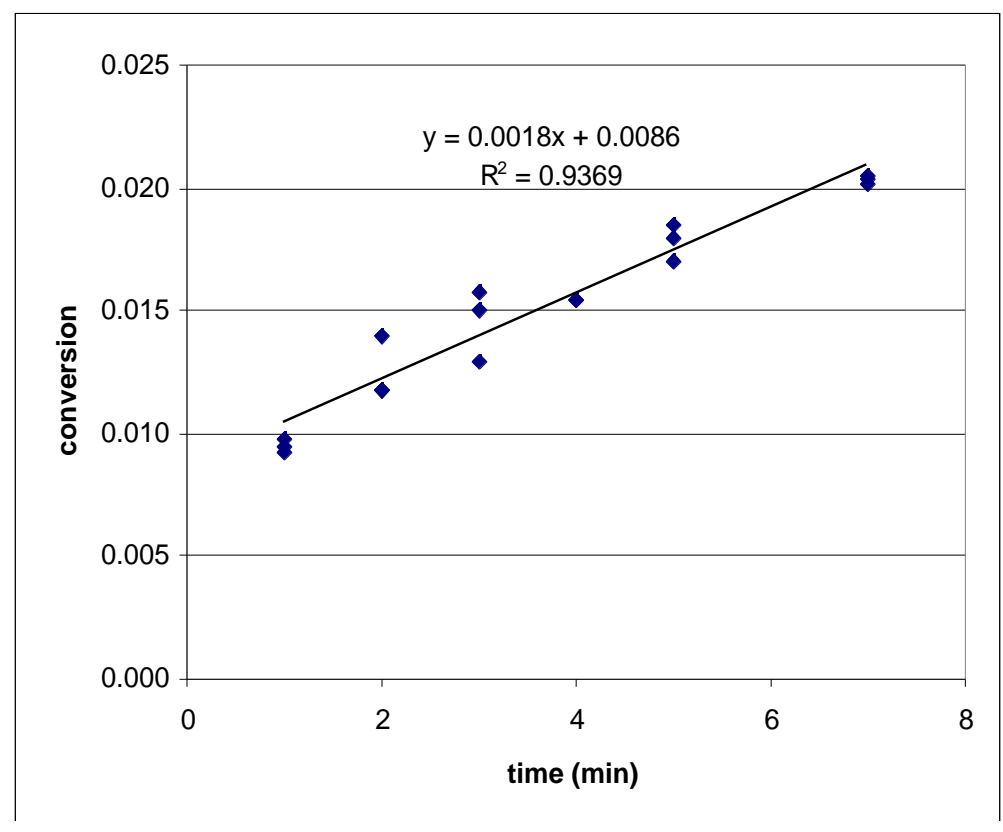




\begin{tabular}{|c|c|c|c|}
\hline time & area $\mathrm{A}$ & area 16 & conversion \\
\hline 1 & 7553 & 527 & 0.009 \\
\hline 1 & 8017 & 579 & 0.010 \\
\hline 1 & 7371 & 500 & 0.009 \\
\hline 2 & 10333 & 1061 & 0.014 \\
\hline 2 & 9903 & 857 & 0.012 \\
\hline 2 & 11809 & 1021 & 0.012 \\
\hline 3 & 6487 & 716 & 0.015 \\
\hline 3 & 7188 & 834 & 0.016 \\
\hline 3 & 7467 & 709 & 0.013 \\
\hline 4 & 5942 & 678 & 0.015 \\
\hline 4 & 7629 & 869 & 0.015 \\
\hline 5 & 5753 & 719 & 0.017 \\
\hline 5 & 5103 & 674 & 0.018 \\
\hline 5 & 6116 & 832 & 0.018 \\
\hline 7 & 7805 & 1175 & 0.020 \\
\hline 7 & 8326 & 1248 & 0.020 \\
\hline 7 & 9465 & 1409 & 0.020 \\
\hline 10 & 3472 & 629 & 0.025 \\
\hline 10 & 3567 & 597 & 0.023 \\
\hline 10 & 3935 & 703 & 0.024 \\
\hline 15 & 3304 & 779 & 0.032 \\
\hline 15 & 3511 & 887 & 0.034 \\
\hline 15 & 4009 & 893 & 0.030 \\
\hline 20 & 3157 & 887 & 0.038 \\
\hline 20 & 3741 & 975 & 0.035 \\
\hline 20 & 4145 & 1070 & 0.035 \\
\hline 30 & 4022 & 1342 & 0.045 \\
\hline 30 & 5270 & 1700 & 0.044 \\
\hline 30 & 5840 & 1906 & 0.044 \\
\hline 40 & 3309 & 1279 & 0.052 \\
\hline 40 & 3629 & 1397 & 0.052 \\
\hline 40 & 4190 & 1621 & 0.053 \\
\hline 50 & 4262 & 1841 & 0.059 \\
\hline 50 & 4506 & 1897 & 0.057 \\
\hline 50 & 4773 & 2009 & 0.057 \\
\hline 60 & 3714 & 1700 & 0.062 \\
\hline 60 & 3906 & 1840 & 0.064 \\
\hline 60 & 3575 & 1706 & 0.065 \\
\hline 75 & 3941 & 2025 & 0.070 \\
\hline 75 & 3644 & 1963 & 0.073 \\
\hline 75 & 4392 & 2355 & 0.073 \\
\hline 90 & 4491 & 2532 & 0.077 \\
\hline 90 & 4604 & 2658 & 0.078 \\
\hline 90 & 4780 & 2725 & 0.077 \\
\hline 120 & 4066 & 2792 & 0.093 \\
\hline 120 & 4447 & 2854 & 0.087 \\
\hline 120 & 4946 & 3175 & 0.087 \\
\hline
\end{tabular}




\section{Order in (E)-1, (0.75 equiv).}

(E)-1

2-Iodothiophene

Tetra- $n$-butylammonium fluoride

Bis(dibenzylideneacetone)palladium $2.5 \mathrm{mM}$ ( 0.05 equiv)
$38 \mathrm{mM}$ ( 0.75 equiv)

$50 \mathrm{mM}$ (1.0 equiv)

$188 \mathrm{mM}$ (3.75 equiv)

Following the General Procedure, silanol (E)-1 (32 mg, $0.188 \mathrm{mmol})$ and biphenyl (4.9 $\mathrm{mg}$ ) were dissolved in TBAF solution $(940 \mu \mathrm{L}, 1.0 \mathrm{M}$ in THF, $0.94 \mathrm{mmol})$ in a flame dried 2neck round-bottomed flask, equipped with a stir bar and a septum under argon. Dry THF (3.05 $\mathrm{mL}$ ) was added and the clear, slightly-yellow solution was immersed into a water-bath at $23{ }^{\circ} \mathrm{C} \pm$ $1{ }^{\circ} \mathrm{C}$ for $30 \mathrm{~min}$. The 2-iodothiophene $(28 \mu \mathrm{L}, 0.25 \mathrm{mmol})$ was then added and the reaction was stirred for $1 \mathrm{~min}$. A suspension of $\operatorname{Pd}(\mathrm{dba})_{2}(7.2 \mathrm{mg}, 0.0125 \mathrm{mmol})$ in dry THF $(1.0 \mathrm{~mL})$ was added. The initially purple, turbid reaction mixture turns into a clear solution within 30 seconds. Aliquots $(\sim 200 \mu \mathrm{L})$ were withdrawn after 1, 2, 3, 4, 5, 7, 10, 15, 20, 30, 40, 50, 60, 75, 90, and $120 \mathrm{~min}$. To quench, these samples were added to $100 \mu \mathrm{L}$ of a $20 \%$ aqueous solution of 2dimethylaminoethanethiol hydrochloride. The clear colorless to slightly-yellow solution was then filtered through a plug of silica gel, and the silica gel was washed with diethyl ether to give a total sample volume of $\sim 2 \mathrm{~mL}$. This sample was injected into the GC three times.

initial rate $=0.0043$

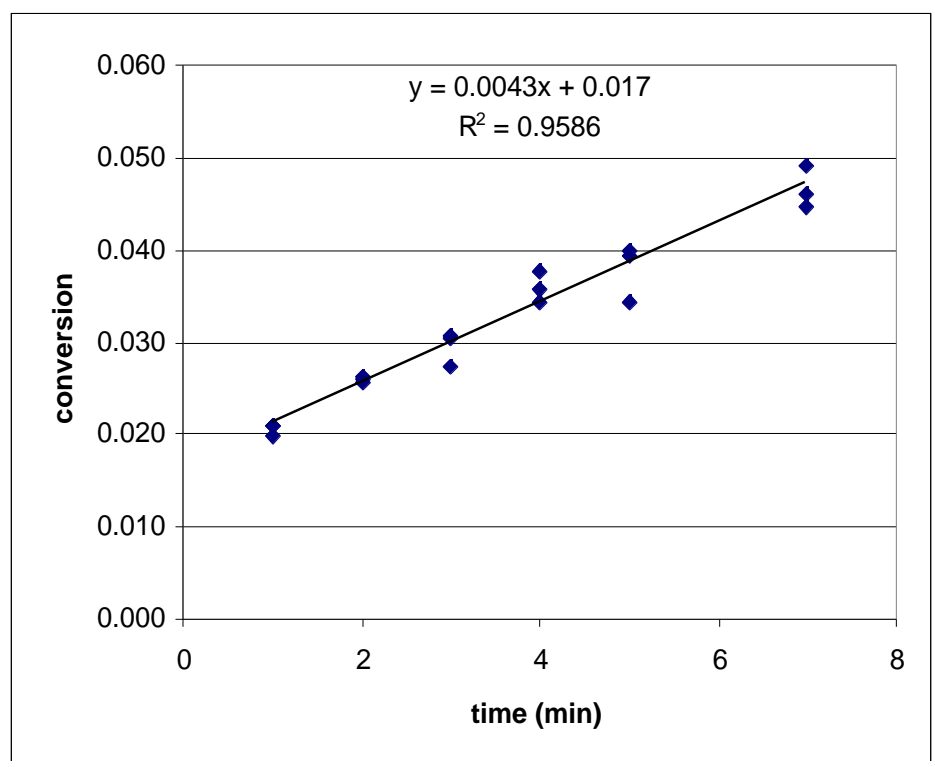




\begin{tabular}{|c|c|c|c|}
\hline time & $\operatorname{area} \mathrm{A}$ & area 16 & conversion \\
\hline 1 & 9960 & 1124 & 0.021 \\
\hline 1 & 12312 & 1325 & 0.020 \\
\hline 1 & 13910 & 1576 & 0.021 \\
\hline 2 & 10531 & 1483 & 0.026 \\
\hline 2 & 15410 & 2188 & 0.026 \\
\hline 2 & 15214 & 2125 & 0.026 \\
\hline 3 & 8295 & 1384 & 0.031 \\
\hline 3 & 10633 & 1573 & 0.027 \\
\hline 3 & 10484 & 1733 & 0.031 \\
\hline 4 & 6887 & 1333 & 0.036 \\
\hline 4 & 8531 & 1735 & 0.038 \\
\hline 4 & 9425 & 1747 & 0.034 \\
\hline 5 & 7688 & 1639 & 0.039 \\
\hline 5 & 8548 & 1586 & 0.034 \\
\hline 5 & 9244 & 1992 & 0.040 \\
\hline 7 & 7071 & 1765 & 0.046 \\
\hline 7 & 4230 & 1025 & 0.045 \\
\hline 7 & 8703 & 2316 & 0.049 \\
\hline 10 & 5868 & 1838 & 0.058 \\
\hline 10 & 7190 & 2201 & 0.057 \\
\hline 10 & 5754 & 1947 & 0.063 \\
\hline 15 & 5811 & 2294 & 0.073 \\
\hline 15 & 5123 & 1977 & 0.071 \\
\hline 15 & 5290 & 2136 & 0.075 \\
\hline 20 & 5754 & 2670 & 0.086 \\
\hline 20 & 6863 & 3164 & 0.085 \\
\hline 20 & 6875 & 3175 & 0.085 \\
\hline 30 & 4828 & 2761 & 0.106 \\
\hline 30 & 5125 & 3026 & 0.109 \\
\hline 30 & 7041 & 3861 & 0.101 \\
\hline 40 & 2896 & 1978 & 0.126 \\
\hline 40 & 3658 & 2524 & 0.128 \\
\hline 40 & 4226 & 2787 & 0.122 \\
\hline 50 & 7102 & 5629 & 0.146 \\
\hline 50 & 9376 & 7380 & 0.145 \\
\hline 50 & 8993 & 7058 & 0.145 \\
\hline 60 & 6226 & 5438 & 0.161 \\
\hline 60 & 5920 & 5326 & 0.166 \\
\hline 60 & 5991 & 5334 & 0.165 \\
\hline 75 & 8680 & 8716 & 0.186 \\
\hline 75 & 9641 & 9828 & 0.188 \\
\hline 75 & 8979 & 9026 & 0.186 \\
\hline 90 & 5835 & 6641 & 0.210 \\
\hline 90 & 6433 & 6795 & 0.195 \\
\hline 90 & 6322 & 6962 & 0.204 \\
\hline 120 & 6650 & 8455 & 0.235 \\
\hline 120 & 7238 & 9400 & 0.240 \\
\hline
\end{tabular}




\section{Order in $(E)-1,(0.75$ equiv $)$.}

(E)-1

2-Iodothiophene

Tetra- $n$-butylammonium fluoride

Bis(dibenzylideneacetone)palladium $2.5 \mathrm{mM}$ (0.05 equiv)
$38 \mathrm{mM}(0.75$ equiv $)$

$50 \mathrm{mM}$ (1.0 equiv)

$188 \mathrm{mM}$ (3.75 equiv)

Following the General Procedure, silanol (E)-1 (32 mg, $0.188 \mathrm{mmol})$ and biphenyl (4.9 $\mathrm{mg}$ ) were dissolved in TBAF solution $(940 \mu \mathrm{L}, 1.0 \mathrm{M}$ in THF, $0.94 \mathrm{mmol})$ in a flame dried 2neck round-bottomed flask, equipped with a stir bar and a septum under argon. Dry THF (3.05 $\mathrm{mL}$ ) was added and the clear, slightly-yellow solution was immersed into a water-bath at $23{ }^{\circ} \mathrm{C} \pm$ $1{ }^{\circ} \mathrm{C}$ for $30 \mathrm{~min}$. The 2-iodothiophene $(28 \mu \mathrm{L}, 0.25 \mathrm{mmol})$ was then added and the reaction was stirred for $1 \mathrm{~min}$. A suspension of $\operatorname{Pd}(\mathrm{dba})_{2}(7.2 \mathrm{mg}, 0.0125 \mathrm{mmol})$ in dry THF $(1.0 \mathrm{~mL})$ was added. The initially purple, turbid reaction mixture turns into a clear solution within 30 seconds. Aliquots $(\sim 200 \mu \mathrm{L})$ were withdrawn after 1, 2, 3, 4, 5, 7, 10, 15, 20, 30, 40, 50, 60, 75, 90, and $120 \mathrm{~min}$. To quench, these samples were added to $100 \mu \mathrm{L}$ of a $20 \%$ aqueous solution of 2dimethylaminoethanethiol hydrochloride. The clear colorless to slightly-yellow solution was then filtered through a plug of silica gel, and the silica gel was washed with diethyl ether to give a total sample volume of $\sim 2 \mathrm{~mL}$. This sample was injected into the GC three times.

initial rate $=0.0036$

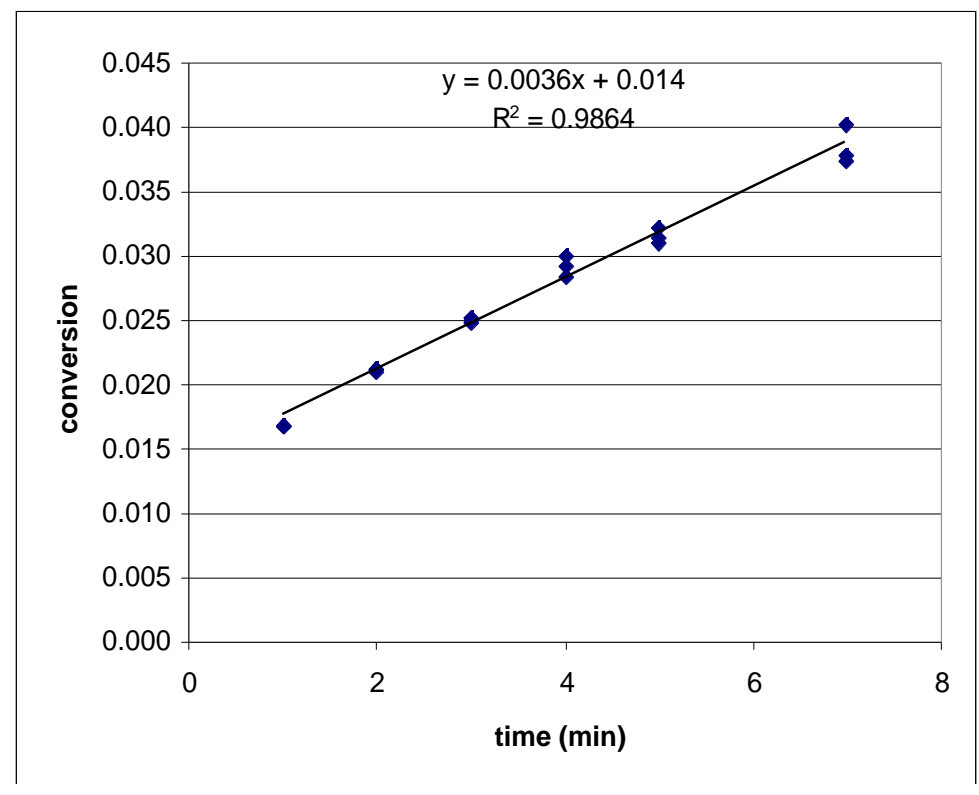




\begin{tabular}{|c|c|c|c|}
\hline time & area $\mathrm{A}$ & area 16 & conversion \\
\hline 1 & 10515 & 959 & 0.017 \\
\hline 1 & 13539 & 1234 & 0.017 \\
\hline 1 & 13273 & 1201 & 0.017 \\
\hline 2 & 11622 & 1330 & 0.021 \\
\hline 2 & 10908 & 1256 & 0.021 \\
\hline 2 & 11418 & 1303 & 0.021 \\
\hline 3 & 8942 & 1196 & 0.025 \\
\hline 3 & 9897 & 1350 & 0.025 \\
\hline 3 & 10284 & 1393 & 0.025 \\
\hline 4 & 11613 & 1835 & 0.029 \\
\hline 4 & 11828 & 1819 & 0.028 \\
\hline 4 & 11348 & 1839 & 0.030 \\
\hline 5 & 10795 & 1815 & 0.031 \\
\hline 5 & 5545 & 965 & 0.032 \\
\hline 5 & 11604 & 1972 & 0.031 \\
\hline 7 & 5003 & 1011 & 0.037 \\
\hline 7 & 6106 & 1328 & 0.040 \\
\hline 7 & 6929 & 1416 & 0.038 \\
\hline 10 & 4071 & 988 & 0.045 \\
\hline 10 & 4612 & 1216 & 0.049 \\
\hline 10 & 5682 & 1494 & 0.049 \\
\hline 15 & 3530 & 1137 & 0.060 \\
\hline 15 & 4543 & 1508 & 0.061 \\
\hline 15 & 4769 & 1283 & 0.050 \\
\hline 20 & 4793 & 1673 & 0.065 \\
\hline 20 & 6807 & 2345 & 0.064 \\
\hline 20 & 6612 & 2365 & 0.066 \\
\hline 30 & 4659 & 2262 & 0.090 \\
\hline 30 & 5996 & 2885 & 0.089 \\
\hline 30 & 6741 & 3194 & 0.088 \\
\hline 40 & 4382 & 2478 & 0.104 \\
\hline 40 & 5705 & 3220 & 0.104 \\
\hline 40 & 5349 & 3095 & 0.107 \\
\hline 50 & 4442 & 2984 & 0.124 \\
\hline 50 & 6133 & 4003 & 0.121 \\
\hline 50 & 6500 & 4460 & 0.127 \\
\hline 60 & 5761 & 4301 & 0.138 \\
\hline 60 & 7453 & 5432 & 0.135 \\
\hline 60 & 7289 & 5531 & 0.140 \\
\hline 75 & 4078 & 3535 & 0.160 \\
\hline 75 & 5383 & 4832 & 0.166 \\
\hline 75 & 4864 & 4655 & 0.177 \\
\hline 90 & 4911 & 4604 & 0.173 \\
\hline 90 & 5675 & 5463 & 0.178 \\
\hline 90 & 5531 & 5328 & 0.178 \\
\hline 120 & 4460 & 5100 & 0.211 \\
\hline 120 & 5967 & 6804 & 0.211 \\
\hline
\end{tabular}




\section{Order in $(E)-1,(0.75$ equiv $)$.}

(E)-1

2-Iodothiophene

Tetra- $n$-butylammonium fluoride

Bis(dibenzylideneacetone)palladium $2.5 \mathrm{mM}$ (0.05 equiv)
$38 \mathrm{mM}(0.75$ equiv $)$

$50 \mathrm{mM}$ (1.0 equiv)

$188 \mathrm{mM}$ (3.75 equiv)

Following the General Procedure, silanol (E)-1 (32 mg, $0.188 \mathrm{mmol})$ and biphenyl (4.3 $\mathrm{mg}$ ) were dissolved in TBAF solution $(940 \mu \mathrm{L}, 1.0 \mathrm{M}$ in THF, $0.94 \mathrm{mmol})$ in a flame dried 2neck round-bottomed flask, equipped with a stir bar and a septum under argon. Dry THF (3.05 $\mathrm{mL}$ ) was added and the clear, slightly-yellow solution was immersed into a water-bath at $23{ }^{\circ} \mathrm{C} \pm$ $1{ }^{\circ} \mathrm{C}$ for $30 \mathrm{~min}$. The 2-iodothiophene $(28 \mu \mathrm{L}, 0.25 \mathrm{mmol})$ was then added and the reaction was stirred for $1 \mathrm{~min}$. A suspension of $\operatorname{Pd}(\mathrm{dba})_{2}(7.2 \mathrm{mg}, 0.0125 \mathrm{mmol})$ in dry THF $(1.0 \mathrm{~mL})$ was added. The initially purple, turbid reaction mixture turns into a clear solution within 30 seconds. Aliquots $(\sim 200 \mu \mathrm{L})$ were withdrawn after 1, 2, 3, 4, 5, 7, 10, 15, 20, 30, 40, 50, 60, 75, 90, and $120 \mathrm{~min}$. To quench, these samples were added to $100 \mu \mathrm{L}$ of a $20 \%$ aqueous solution of 2dimethylaminoethanethiol hydrochloride. The clear colorless to slightly-yellow solution was then filtered through a plug of silica gel, and the silica gel was washed with diethyl ether to give a total sample volume of $\sim 2 \mathrm{~mL}$. This sample was injected into the GC three times.

initial rate $=0.004$

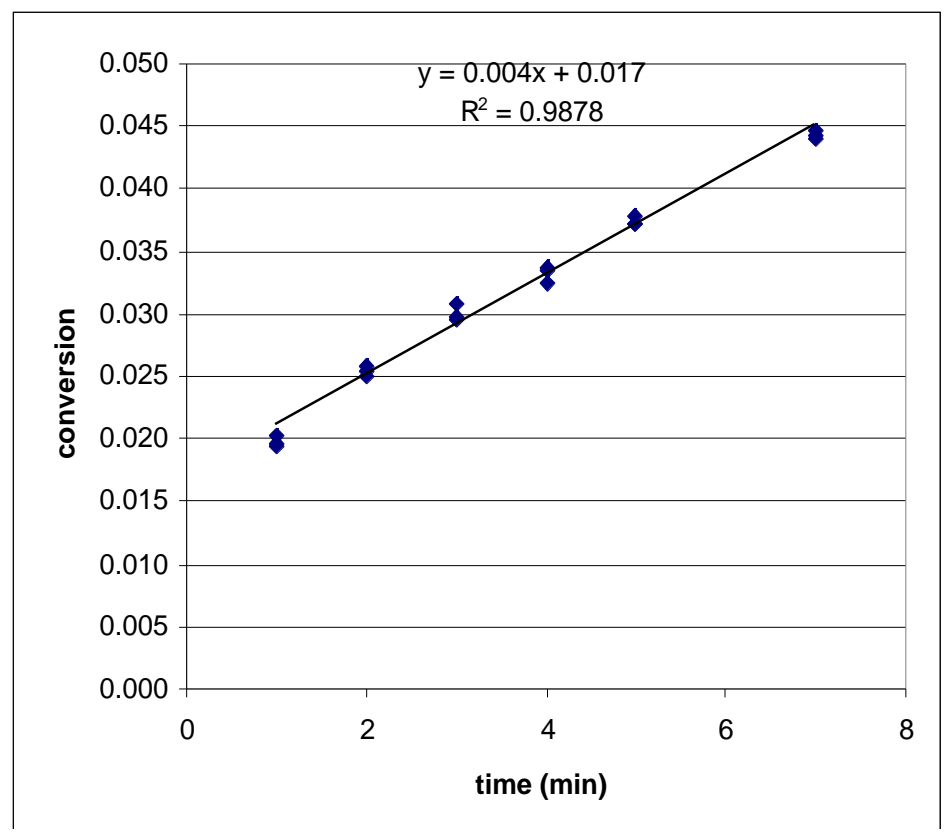




\begin{tabular}{|c|c|c|c|}
\hline time & area $\mathrm{A}$ & area 16 & conversion \\
\hline 1 & 9824 & 1223 & 0.020 \\
\hline 1 & 7927 & 960 & 0.020 \\
\hline 1 & 8059 & 964 & 0.019 \\
\hline 2 & 7883 & 1257 & 0.026 \\
\hline 2 & 6999 & 1098 & 0.025 \\
\hline 2 & 6180 & 952 & 0.025 \\
\hline 3 & 7550 & 1387 & 0.030 \\
\hline 3 & 6219 & 1182 & 0.031 \\
\hline 3 & 6786 & 1237 & 0.030 \\
\hline 4 & 7547 & 1553 & 0.033 \\
\hline 4 & 7671 & 1535 & 0.032 \\
\hline 4 & 7667 & 1594 & 0.034 \\
\hline 5 & 7443 & 1710 & 0.037 \\
\hline 5 & 6910 & 1608 & 0.038 \\
\hline 5 & 6614 & 1513 & 0.037 \\
\hline 7 & 8512 & 2343 & 0.045 \\
\hline 7 & 7577 & 2063 & 0.044 \\
\hline 7 & 6950 & 1882 & 0.044 \\
\hline 10 & 5043 & 1663 & 0.053 \\
\hline 10 & 4652 & 1524 & 0.053 \\
\hline 10 & 4309 & 1428 & 0.054 \\
\hline 15 & 5511 & 2301 & 0.068 \\
\hline 15 & 5519 & 2346 & 0.069 \\
\hline 15 & 4963 & 2092 & 0.068 \\
\hline 20 & 3318 & 1563 & 0.076 \\
\hline 20 & 3379 & 1713 & 0.082 \\
\hline 20 & 3207 & 1677 & 0.085 \\
\hline 30 & 3253 & 2058 & 0.103 \\
\hline 30 & 3397 & 2114 & 0.101 \\
\hline 30 & 2655 & 1654 & 0.101 \\
\hline 40 & 3991 & 2903 & 0.118 \\
\hline 40 & 3530 & 2664 & 0.122 \\
\hline 40 & 3216 & 2373 & 0.120 \\
\hline 50 & 4461 & 3749 & 0.136 \\
\hline 50 & 4100 & 3487 & 0.138 \\
\hline 50 & 3564 & 3021 & 0.137 \\
\hline 60 & 4597 & 4302 & 0.152 \\
\hline 60 & 4692 & 4391 & 0.152 \\
\hline 60 & 4684 & 4357 & 0.151 \\
\hline 75 & 4496 & 4845 & 0.175 \\
\hline 75 & 4230 & 4487 & 0.172 \\
\hline 75 & 3921 & 4291 & 0.177 \\
\hline 90 & 5248 & 6151 & 0.190 \\
\hline 90 & 4639 & 5359 & 0.187 \\
\hline 90 & 4526 & 5330 & 0.191 \\
\hline 120 & 3357 & 4633 & 0.224 \\
\hline 120 & 3952 & 5395 & 0.221 \\
\hline
\end{tabular}




\section{Order in $(E)-1,(1.0$ equiv).}

(E)-1

2-Iodothiophene

Tetra- $n$-butylammonium fluoride

Bis(dibenzylideneacetone)palladium $2.5 \mathrm{mM}$ (0.05 equiv)
$50 \mathrm{mM}$ (1.0 equiv)

$50 \mathrm{mM}$ (1.0 equiv)

$188 \mathrm{mM}$ (3.75 equiv)

Following the General Procedure, silanol (E)-1 (43 mg, $0.25 \mathrm{mmol})$ and biphenyl (10.5 $\mathrm{mg}$ ) were dissolved in TBAF solution $(940 \mu \mathrm{L}, 1.0 \mathrm{M}$ in THF, $0.94 \mathrm{mmol})$ in a flame dried 2neck round-bottomed flask, equipped with a stir bar and a septum under argon. Dry THF (3.05 $\mathrm{mL}$ ) was added and the clear, slightly-yellow solution was immersed into a water-bath at $23{ }^{\circ} \mathrm{C} \pm$ $1{ }^{\circ} \mathrm{C}$ for $30 \mathrm{~min}$. The 2-iodothiophene $(28 \mu \mathrm{L}, 0.25 \mathrm{mmol})$ was then added and the reaction was stirred for $1 \mathrm{~min}$. A suspension of $\operatorname{Pd}(\mathrm{dba})_{2}(7.2 \mathrm{mg}, 0.0125 \mathrm{mmol})$ in dry THF $(1.0 \mathrm{~mL})$ was added. The initially purple, turbid reaction mixture turns into a clear solution within 30 seconds. Aliquots $(\sim 200 \mu \mathrm{L})$ were withdrawn after 1, 2, 3, 4, 5, 7, 10, 15, 20, 30, 40, 50, 60, 75, 90, and $120 \mathrm{~min}$. To quench, these samples were added to $100 \mu \mathrm{L}$ of a $20 \%$ aqueous solution of 2dimethylaminoethanethiol hydrochloride. The clear colorless to slightly-yellow solution was then filtered through a plug of silica gel, and the silica gel was washed with diethyl ether to give a total sample volume of $\sim 2 \mathrm{~mL}$. This sample was injected into the GC three times.

initial rate $=0.0094$

$\mathrm{k}_{\text {obs }}=0.0026$
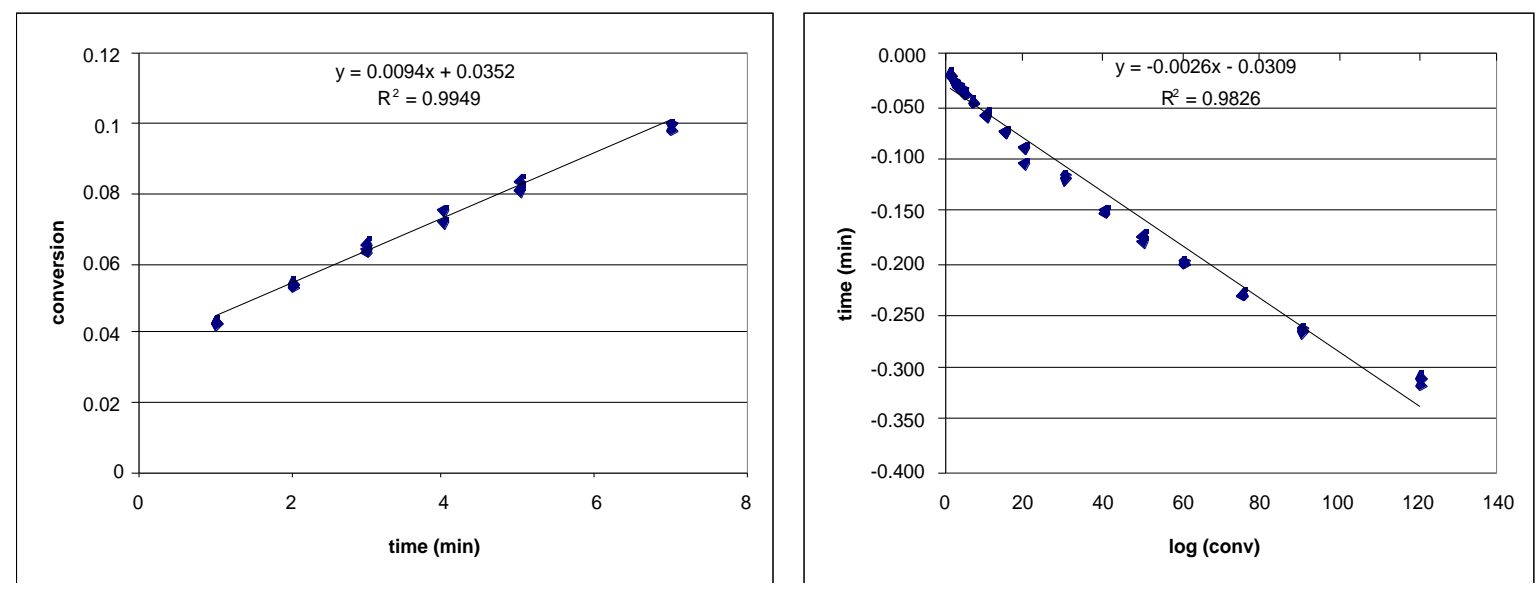


\begin{tabular}{|c|c|c|c|c|}
\hline time & area $\mathrm{A}$ & area 16 & conversion & $\log (1-\mathrm{conv})$ \\
\hline 1 & 6725 & 735 & 0.043 & -0.019 \\
\hline 1 & 7165 & 788 & 0.044 & -0.019 \\
\hline 1 & 8101 & 877 & 0.043 & -0.019 \\
\hline 2 & 5000 & 690 & 0.055 & -0.024 \\
\hline 2 & 5326 & 722 & 0.054 & -0.024 \\
\hline 2 & 5380 & 731 & 0.054 & -0.024 \\
\hline 3 & 6016 & 965 & 0.064 & -0.029 \\
\hline 3 & 6402 & 1041 & 0.064 & -0.029 \\
\hline 3 & 6682 & 1109 & 0.066 & -0.030 \\
\hline 4 & 5617 & 1073 & 0.076 & -0.034 \\
\hline 4 & 6496 & 1187 & 0.072 & -0.033 \\
\hline 5 & 8273 & 1756 & 0.084 & -0.038 \\
\hline 5 & 8892 & 1817 & 0.081 & -0.037 \\
\hline 5 & 9681 & 2002 & 0.082 & -0.037 \\
\hline 7 & 6705 & 1700 & 0.100 & -0.046 \\
\hline 7 & 7026 & 1753 & 0.099 & -0.045 \\
\hline 7 & 7151 & 1813 & 0.100 & -0.046 \\
\hline 10 & 6691 & 2104 & 0.125 & -0.058 \\
\hline 10 & 7282 & 2239 & 0.122 & -0.056 \\
\hline 10 & 7907 & 2487 & 0.125 & -0.058 \\
\hline 15 & 6182 & 2441 & 0.156 & -0.074 \\
\hline 15 & 6620 & 2585 & 0.155 & -0.073 \\
\hline 15 & 7229 & 2823 & 0.155 & -0.073 \\
\hline 20 & 7093 & 3815 & 0.213 & -0.104 \\
\hline 20 & 7695 & 3571 & 0.184 & -0.088 \\
\hline 20 & 8405 & 3904 & 0.184 & -0.088 \\
\hline 30 & 6900 & 4165 & 0.239 & -0.119 \\
\hline 30 & 7627 & 4434 & 0.230 & -0.114 \\
\hline 30 & 8816 & 5332 & 0.239 & -0.119 \\
\hline 40 & 6677 & 4881 & 0.289 & -0.148 \\
\hline 40 & 7292 & 5393 & 0.293 & -0.150 \\
\hline 40 & 8489 & 6237 & 0.291 & -0.149 \\
\hline 50 & 6209 & 5279 & 0.337 & -0.178 \\
\hline 50 & 7149 & 5918 & 0.328 & -0.173 \\
\hline 50 & 7404 & 6112 & 0.327 & -0.172 \\
\hline 60 & 7439 & 6857 & 0.365 & -0.197 \\
\hline 60 & 8409 & 7834 & 0.369 & -0.200 \\
\hline 60 & 8717 & 8078 & 0.367 & -0.199 \\
\hline 75 & 5147 & 5317 & 0.409 & -0.228 \\
\hline 75 & 5908 & 6107 & 0.409 & -0.229 \\
\hline 75 & 5766 & 5987 & 0.411 & -0.230 \\
\hline 90 & 6949 & 7942 & 0.453 & -0.262 \\
\hline 90 & 6313 & 7240 & 0.454 & -0.263 \\
\hline 90 & 7772 & 8963 & 0.457 & -0.265 \\
\hline 120 & 6152 & 8023 & 0.516 & -0.316 \\
\hline 120 & 6397 & 8256 & 0.511 & -0.311 \\
\hline 120 & 8107 & 10412 & 0.509 & -0.309 \\
\hline
\end{tabular}




\section{Order in $(E)-1,(1.0$ equiv $)$.}

(E)-1

2-Iodothiophene

Tetra- $n$-butylammonium fluoride

Bis(dibenzylideneacetone)palladium $2.5 \mathrm{mM}$ (0.05 equiv)
$50 \mathrm{mM}$ (1.0 equiv)

$50 \mathrm{mM}$ (1.0 equiv)

$188 \mathrm{mM}$ (3.75 equiv)

Following the General Procedure, silanol (E)-1 (43 mg, $0.25 \mathrm{mmol})$ and biphenyl (10.1 $\mathrm{mg}$ ) were dissolved in TBAF solution $(940 \mu \mathrm{L}, 1.0 \mathrm{M}$ in THF, $0.94 \mathrm{mmol})$ in a flame dried 2neck round-bottomed flask, equipped with a stir bar and a septum under argon. Dry THF (3.05 $\mathrm{mL}$ ) was added and the clear, slightly-yellow solution was immersed into a water-bath at $23{ }^{\circ} \mathrm{C} \pm$ $1{ }^{\circ} \mathrm{C}$ for $30 \mathrm{~min}$. The 2-iodothiophene $(28 \mu \mathrm{L}, 0.25 \mathrm{mmol})$ was then added and the reaction was stirred for $1 \mathrm{~min}$. A suspension of $\mathrm{Pd}(\mathrm{dba})_{2}(7.2 \mathrm{mg}, 0.0125 \mathrm{mmol})$ in dry THF $(1.0 \mathrm{~mL})$ was added. The initially purple, turbid reaction mixture turns into a clear solution within 30 seconds. Aliquots $(\sim 200 \mu \mathrm{L})$ were withdrawn after 1, 2, 3, 4, 5, 7, 10, 15, 20, 30, 40, 50, 60, 75, 90, and $120 \mathrm{~min}$. To quench, these samples were added to $100 \mu \mathrm{L}$ of a $20 \%$ aqueous solution of 2dimethylaminoethanethiol hydrochloride. The clear colorless to slightly-yellow solution was then filtered through a plug of silica gel, and the silica gel was washed with diethyl ether to give a total sample volume of $\sim 2 \mathrm{~mL}$. This sample was injected into the GC three times.

initial rate $=0.0112$

$\mathrm{k}_{\mathrm{obs}}=0.0043$
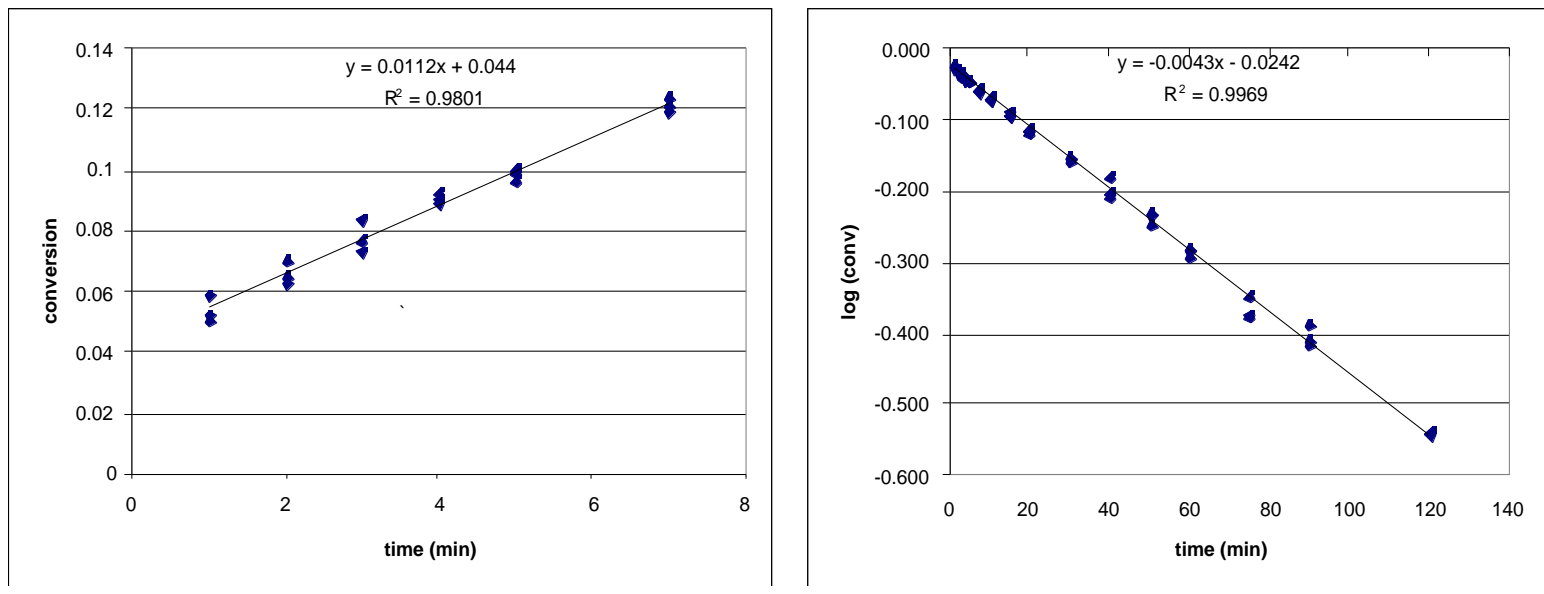


\begin{tabular}{|c|c|c|c|c|}
\hline time & area $\mathrm{A}$ & area 16 & conversion & $\log (1-\mathrm{conv})$ \\
\hline 1 & 4056 & 562 & 0.053 & -0.024 \\
\hline 1 & 4861 & 643 & 0.050 & -0.022 \\
\hline 1 & 5433 & 840 & 0.059 & -0.026 \\
\hline 2 & 4628 & 770 & 0.063 & -0.028 \\
\hline 2 & 7033 & 1200 & 0.065 & -0.029 \\
\hline 2 & 5746 & 1065 & 0.071 & -0.032 \\
\hline 3 & 4341 & 838 & 0.074 & -0.033 \\
\hline 3 & 6230 & 1263 & 0.077 & -0.035 \\
\hline 3 & 5007 & 1108 & 0.084 & -0.038 \\
\hline 4 & 3732 & 909 & 0.093 & -0.042 \\
\hline 4 & 5072 & 1205 & 0.090 & -0.041 \\
\hline 4 & 4594 & 1082 & 0.090 & -0.041 \\
\hline 5 & 4962 & 1259 & 0.097 & -0.044 \\
\hline 5 & 6751 & 1755 & 0.099 & -0.045 \\
\hline 5 & 6437 & 1702 & 0.101 & -0.046 \\
\hline 7 & 3118 & 1016 & 0.124 & -0.058 \\
\hline 7 & 4442 & 1417 & 0.122 & -0.056 \\
\hline 7 & 4348 & 1363 & 0.119 & -0.055 \\
\hline 10 & 3819 & 1478 & 0.147 & -0.069 \\
\hline 10 & 5586 & 2102 & 0.143 & -0.067 \\
\hline 10 & 5611 & 2176 & 0.148 & -0.069 \\
\hline 15 & 3477 & 1740 & 0.191 & -0.092 \\
\hline 15 & 4591 & 2189 & 0.182 & -0.087 \\
\hline 15 & 5281 & 2680 & 0.193 & -0.093 \\
\hline 20 & 4527 & 2712 & 0.228 & -0.112 \\
\hline 20 & 5840 & 3579 & 0.233 & -0.115 \\
\hline 20 & 5775 & 3630 & 0.239 & -0.119 \\
\hline 30 & 4171 & 3199 & 0.292 & -0.150 \\
\hline 30 & 5536 & 4375 & 0.301 & -0.156 \\
\hline 30 & 5632 & 4461 & 0.302 & -0.156 \\
\hline 40 & 4490 & 4014 & 0.341 & -0.181 \\
\hline 40 & 4761 & 4734 & 0.379 & -0.207 \\
\hline 40 & 4990 & 4854 & 0.371 & -0.201 \\
\hline 50 & 3884 & 4175 & 0.409 & -0.229 \\
\hline 50 & 4945 & 5382 & 0.415 & -0.233 \\
\hline 50 & 4910 & 5565 & 0.432 & -0.245 \\
\hline 60 & 4924 & 6160 & 0.477 & -0.281 \\
\hline 60 & 5922 & 7589 & 0.488 & -0.291 \\
\hline 60 & 6113 & 7703 & 0.480 & -0.284 \\
\hline 75 & 3942 & 5955 & 0.575 & -0.372 \\
\hline 75 & 4564 & 6935 & 0.579 & -0.375 \\
\hline 75 & 5161 & 7469 & 0.551 & -0.348 \\
\hline 90 & 4925 & 7884 & 0.610 & -0.409 \\
\hline 90 & 6181 & 9966 & 0.614 & -0.414 \\
\hline 90 & 5347 & 8279 & 0.590 & -0.387 \\
\hline 120 & 4737 & 8850 & 0.712 & -0.540 \\
\hline 120 & 6315 & 11773 & 0.710 & -0.538 \\
\hline
\end{tabular}




\section{Order in (E)-1, (1.0 equiv).}

(E)-1

2-Iodothiophene

Tetra- $n$-butylammonium fluoride

Bis(dibenzylideneacetone)palladium $2.5 \mathrm{mM}$ (0.05 equiv)
$50 \mathrm{mM}$ (1.0 equiv)

$50 \mathrm{mM}$ (1.0 equiv)

$188 \mathrm{mM}$ (3.75 equiv)

Following the General Procedure, silanol (E)-1 (43 mg, $0.25 \mathrm{mmol})$ and biphenyl (5.8 $\mathrm{mg}$ ) were dissolved in TBAF solution $(940 \mu \mathrm{L}, 1.0 \mathrm{M}$ in THF, $0.94 \mathrm{mmol})$ in a flame dried 2neck round-bottomed flask, equipped with a stir bar and a septum under argon. Dry THF (3.05 $\mathrm{mL}$ ) was added and the clear, slightly-yellow solution was immersed into a water-bath at $23{ }^{\circ} \mathrm{C} \pm$ $1{ }^{\circ} \mathrm{C}$ for $30 \mathrm{~min}$. The 2-iodothiophene $(28 \mu \mathrm{L}, 0.25 \mathrm{mmol})$ was then added and the reaction was stirred for $1 \mathrm{~min}$. A suspension of $\operatorname{Pd}(\mathrm{dba})_{2}(7.2 \mathrm{mg}, 0.0125 \mathrm{mmol})$ in dry THF $(1.0 \mathrm{~mL})$ was added. The initially purple, turbid reaction mixture turns into a clear solution within 30 seconds. Aliquots $(\sim 200 \mu \mathrm{L})$ were withdrawn after 1, 2, 3, 4, 5, 7, 10, 15, 20, 30, 40, 50, 60, 75, 90, and $120 \mathrm{~min}$. To quench, these samples were added to $100 \mu \mathrm{L}$ of a $20 \%$ aqueous solution of 2dimethylaminoethanethiol hydrochloride. The clear colorless to slightly-yellow solution was then filtered through a plug of silica gel, and the silica gel was washed with diethyl ether to give a total sample volume of $\sim 2 \mathrm{~mL}$. This sample was injected into the GC three times.

initial rate $=0.0104$

$\mathrm{k}_{\text {obs }}=0.0029$
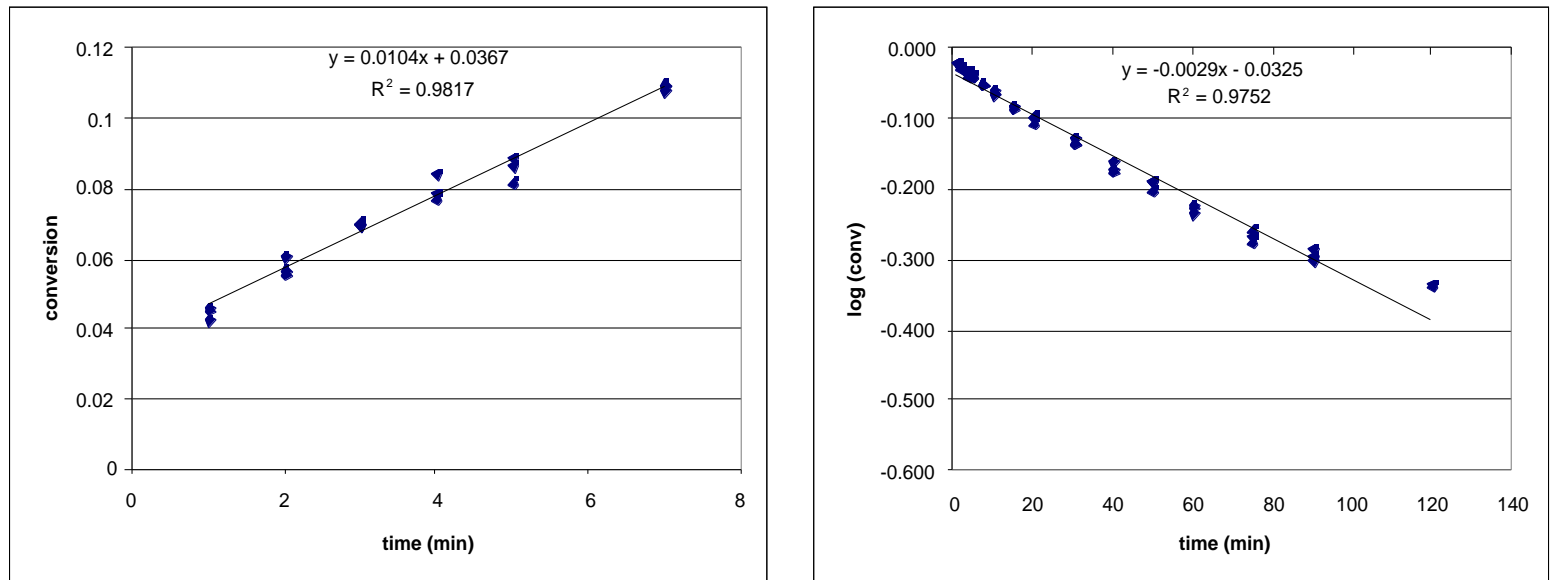


\begin{tabular}{|c|c|c|c|c|}
\hline time & area $\mathrm{A}$ & area $\mathbf{1 6}$ & conversion & $\log (1-\mathrm{conv})$ \\
\hline 1 & 3686 & 726 & 0.043 & -0.019 \\
\hline 1 & 3248 & 687 & 0.046 & -0.021 \\
\hline 1 & 3401 & 706 & 0.045 & -0.020 \\
\hline 2 & 3987 & 1037 & 0.057 & -0.025 \\
\hline 2 & 3608 & 920 & 0.056 & -0.025 \\
\hline 2 & 4021 & 1119 & 0.061 & -0.027 \\
\hline 3 & 4095 & 1315 & 0.070 & -0.032 \\
\hline 3 & 3709 & 1188 & 0.070 & -0.032 \\
\hline 3 & 4422 & 1411 & 0.070 & -0.031 \\
\hline 4 & 3620 & 1282 & 0.077 & -0.035 \\
\hline 4 & 3298 & 1275 & 0.085 & -0.038 \\
\hline 4 & 2904 & 1048 & 0.079 & -0.036 \\
\hline 5 & 3637 & 1484 & 0.089 & -0.041 \\
\hline 5 & 4109 & 1625 & 0.087 & -0.039 \\
\hline 5 & 3982 & 1492 & 0.082 & -0.037 \\
\hline 7 & 2962 & 1467 & 0.108 & -0.050 \\
\hline 7 & 3233 & 1621 & 0.110 & -0.050 \\
\hline 7 & 3603 & 1799 & 0.109 & -0.050 \\
\hline 10 & 3502 & 2047 & 0.128 & -0.059 \\
\hline 10 & 3750 & 2248 & 0.131 & -0.061 \\
\hline 10 & 3741 & 2316 & 0.135 & -0.063 \\
\hline 15 & 3841 & 2990 & 0.170 & -0.081 \\
\hline 15 & 4491 & 3460 & 0.169 & -0.080 \\
\hline 15 & 4500 & 3623 & 0.176 & -0.084 \\
\hline 20 & 3739 & 3419 & 0.200 & -0.097 \\
\hline 20 & 4147 & 3716 & 0.196 & -0.095 \\
\hline 20 & 4050 & 4042 & 0.218 & -0.107 \\
\hline 30 & 3330 & 4077 & 0.268 & -0.135 \\
\hline 30 & 3460 & 4004 & 0.253 & -0.127 \\
\hline 30 & 3554 & 4320 & 0.266 & -0.134 \\
\hline 40 & 3838 & 5813 & 0.331 & -0.175 \\
\hline 40 & 4273 & 6049 & 0.310 & -0.161 \\
\hline 40 & 4089 & 6070 & 0.325 & -0.171 \\
\hline 50 & 3572 & 5762 & 0.353 & -0.189 \\
\hline 50 & 4249 & 6847 & 0.352 & -0.189 \\
\hline 50 & 4372 & 7418 & 0.371 & -0.201 \\
\hline 60 & 4219 & 7782 & 0.403 & -0.224 \\
\hline 60 & 4489 & 8172 & 0.398 & -0.221 \\
\hline 60 & 4451 & 8471 & 0.416 & -0.234 \\
\hline 75 & 4109 & 8799 & 0.468 & -0.274 \\
\hline 75 & 4861 & 9962 & 0.448 & -0.258 \\
\hline 75 & 5558 & 11676 & 0.460 & -0.267 \\
\hline 90 & 5253 & 11809 & 0.492 & -0.294 \\
\hline 90 & 5764 & 12647 & 0.480 & -0.284 \\
\hline 90 & 5986 & 13694 & 0.500 & -0.301 \\
\hline 120 & 4130 & 10198 & 0.540 & -0.337 \\
\hline 120 & 4861 & 11931 & 0.537 & -0.334 \\
\hline
\end{tabular}




\section{Order in $(E)-1,(1.25$ equiv $)$.}

(E)-1

2-Iodothiophene

Tetra- $n$-butylammonium fluoride

Bis(dibenzylideneacetone)palladium $2.5 \mathrm{mM}$ (0.05 equiv)
$63 \mathrm{mM}(1.25$ equiv)

$50 \mathrm{mM}$ (1.0 equiv)

$188 \mathrm{mM}$ (3.75 equiv)

Following the General Procedure, silanol (E)-1 (56 mg, $0.32 \mathrm{mmol})$ and biphenyl (12.0 $\mathrm{mg}$ ) were dissolved in TBAF solution $(940 \mu \mathrm{L}, 1.0 \mathrm{M}$ in THF, $0.94 \mathrm{mmol})$ in a flame dried 2neck round-bottomed flask, equipped with a stir bar and a septum under argon. Dry THF (3.05 $\mathrm{mL}$ ) was added and the clear, slightly-yellow solution was immersed into a water-bath at $23{ }^{\circ} \mathrm{C} \pm$ $1{ }^{\circ} \mathrm{C}$ for $30 \mathrm{~min}$. The 2-iodothiophene $(28 \mu \mathrm{L}, 0.25 \mathrm{mmol})$ was then added and the reaction was stirred for $1 \mathrm{~min}$. A suspension of $\operatorname{Pd}(\mathrm{dba})_{2}(7.2 \mathrm{mg}, 0.0125 \mathrm{mmol})$ in dry THF $(1.0 \mathrm{~mL})$ was added. The initially purple, turbid reaction mixture turns into a clear solution within 30 seconds. Aliquots $(\sim 200 \mu \mathrm{L})$ were withdrawn after 1, 2, 3, 4, 5, 7, 10, 15, 20, 30, 40, 50, 60, 75, 90, and $120 \mathrm{~min}$. To quench, these samples were added to $100 \mu \mathrm{L}$ of a $20 \%$ aqueous solution of 2dimethylaminoethanethiol hydrochloride. The clear colorless to slightly-yellow solution was then filtered through a plug of silica gel, and the silica gel was washed with diethyl ether to give a total sample volume of $\sim 2 \mathrm{~mL}$. This sample was injected into the GC three times.

initial rate $=0.0138$

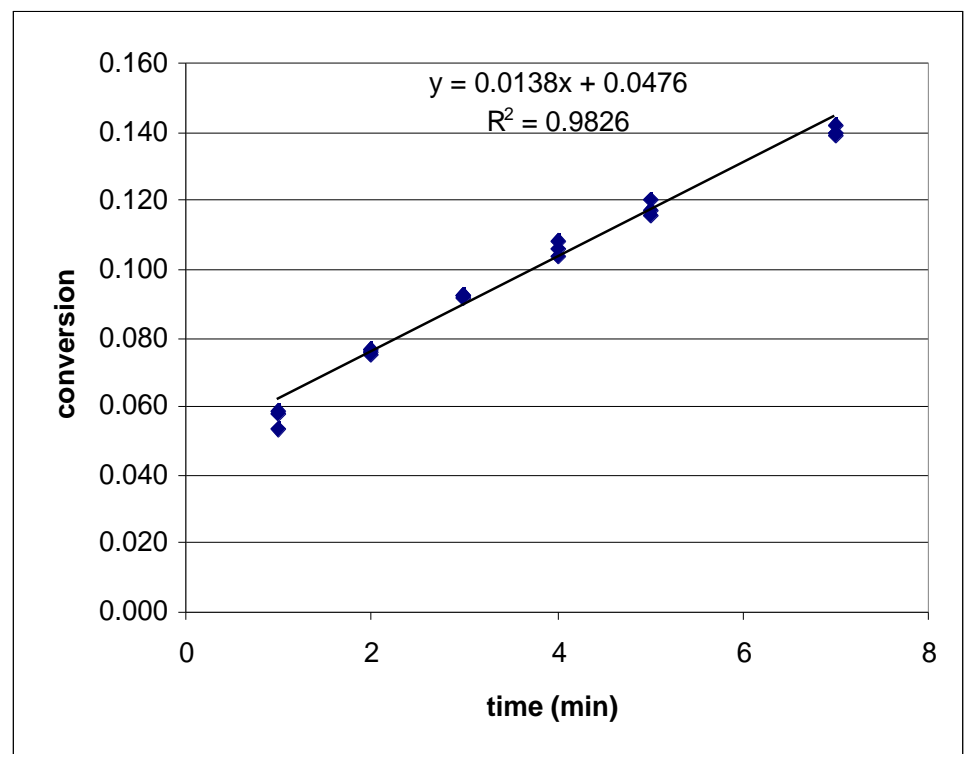




\begin{tabular}{|c|c|c|c|}
\hline time & area $\mathrm{A}$ & area 16 & conversion \\
\hline 1 & 8307 & 1071 & 0.058 \\
\hline 1 & 6950 & 818 & 0.053 \\
\hline 1 & 8479 & 1082 & 0.058 \\
\hline 2 & 7286 & 1235 & 0.077 \\
\hline 2 & 7048 & 1166 & 0.075 \\
\hline 2 & 6479 & 1084 & 0.076 \\
\hline 3 & 15128 & 3095 & 0.093 \\
\hline 3 & 15537 & 3166 & 0.092 \\
\hline 3 & 12774 & 2594 & 0.092 \\
\hline 4 & 6635 & 1554 & 0.106 \\
\hline 4 & 6484 & 1490 & 0.104 \\
\hline 4 & 5952 & 1427 & 0.109 \\
\hline 5 & 8131 & 2076 & 0.116 \\
\hline 5 & 8485 & 2195 & 0.117 \\
\hline 5 & 9693 & 2579 & 0.120 \\
\hline 7 & 9813 & 3016 & 0.139 \\
\hline 7 & 8485 & 2617 & 0.140 \\
\hline 7 & 9180 & 2881 & 0.142 \\
\hline 10 & 6420 & 2443 & 0.172 \\
\hline 10 & 5918 & 2255 & 0.172 \\
\hline 10 & 7224 & 2830 & 0.177 \\
\hline 15 & 7819 & 3856 & 0.223 \\
\hline 15 & 7246 & 3696 & 0.231 \\
\hline 15 & 7810 & 3981 & 0.231 \\
\hline 20 & 9145 & 5573 & 0.276 \\
\hline 20 & 7275 & 4363 & 0.271 \\
\hline 20 & 9323 & 5785 & 0.281 \\
\hline 30 & 8410 & 6739 & 0.363 \\
\hline 30 & 6099 & 4829 & 0.358 \\
\hline 30 & 8252 & 6685 & 0.367 \\
\hline 40 & 11362 & 11024 & 0.439 \\
\hline 40 & 7589 & 7328 & 0.437 \\
\hline 40 & 8690 & 8703 & 0.453 \\
\hline 50 & 9951 & 11324 & 0.515 \\
\hline 50 & 9094 & 10686 & 0.532 \\
\hline 50 & 8404 & 9735 & 0.524 \\
\hline 60 & 10689 & 13560 & 0.574 \\
\hline 60 & 9539 & 12216 & 0.580 \\
\hline 60 & 11721 & 15136 & 0.584 \\
\hline 75 & 8904 & 12610 & 0.641 \\
\hline 75 & 8815 & 12905 & 0.663 \\
\hline 75 & 10493 & 15371 & 0.663 \\
\hline 90 & 10799 & 17370 & 0.728 \\
\hline 90 & 11184 & 18090 & 0.732 \\
\hline 90 & 12930 & 20926 & 0.732 \\
\hline 120 & 10600 & 19495 & 0.832 \\
\hline 120 & 9339 & 17258 & 0.836 \\
\hline
\end{tabular}




\section{Order in $(E)$-1, (1.25 equiv).}

(E)-1

2-Iodothiophene

Tetra- $n$-butylammonium fluoride

Bis(dibenzylideneacetone)palladium $2.5 \mathrm{mM}$ (0.05 equiv)
$63 \mathrm{mM}(1.25$ equiv)

$50 \mathrm{mM}$ (1.0 equiv)

$188 \mathrm{mM}$ (3.75 equiv)

Following the General Procedure, silanol (E)-1 (56 mg, $0.32 \mathrm{mmol})$ and biphenyl (11.6 $\mathrm{mg}$ ) were dissolved in TBAF solution $(940 \mu \mathrm{L}, 1.0 \mathrm{M}$ in THF, $0.94 \mathrm{mmol})$ in a flame dried 2neck round-bottomed flask, equipped with a stir bar and a septum under argon. Dry THF (3.05 $\mathrm{mL}$ ) was added and the clear, slightly-yellow solution was immersed into a water-bath at $23{ }^{\circ} \mathrm{C} \pm$ $1{ }^{\circ} \mathrm{C}$ for $30 \mathrm{~min}$. The 2-iodothiophene $(28 \mu \mathrm{L}, 0.25 \mathrm{mmol})$ was then added and the reaction was stirred for $1 \mathrm{~min}$. A suspension of $\operatorname{Pd}(\mathrm{dba})_{2}(7.2 \mathrm{mg}, 0.0125 \mathrm{mmol})$ in dry THF $(1.0 \mathrm{~mL})$ was added. The initially purple, turbid reaction mixture turns into a clear solution within 30 seconds. Aliquots $(\sim 200 \mu \mathrm{L})$ were withdrawn after 1, 2, 3, 4, 5, 7, 10, 15, 20, 30, 40, 50, 60, 75, 90, and $120 \mathrm{~min}$. To quench, these samples were added to $100 \mu \mathrm{L}$ of a $20 \%$ aqueous solution of 2dimethylaminoethanethiol hydrochloride. The clear colorless to slightly-yellow solution was then filtered through a plug of silica gel, and the silica gel was washed with diethyl ether to give a total sample volume of $\sim 2 \mathrm{~mL}$. This sample was injected into the GC three times.

initial rate $=0.0134$

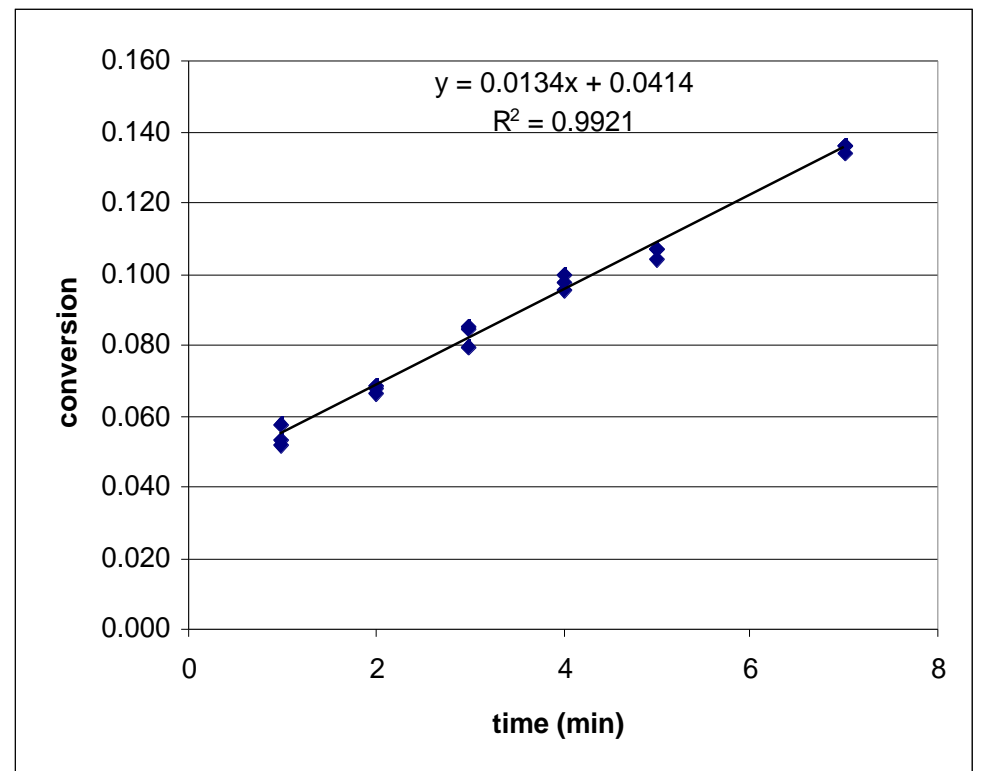




\begin{tabular}{|c|c|c|c|}
\hline Time & $\operatorname{area} \mathrm{A}$ & area 16 & conversion \\
\hline 1 & 8132 & 962 & 0.052 \\
\hline 1 & 7017 & 853 & 0.053 \\
\hline 1 & 8037 & 1052 & 0.057 \\
\hline 2 & 12752 & 1980 & 0.068 \\
\hline 2 & 11874 & 1863 & 0.069 \\
\hline 2 & 10554 & 1588 & 0.066 \\
\hline 3 & 10478 & 1900 & 0.079 \\
\hline 3 & 10385 & 2018 & 0.085 \\
\hline 3 & 10097 & 1942 & 0.084 \\
\hline 4 & 9147 & 1985 & 0.095 \\
\hline 4 & 9241 & 2103 & 0.100 \\
\hline 4 & 10048 & 2240 & 0.098 \\
\hline 5 & 9658 & 2354 & 0.107 \\
\hline 5 & 7297 & 1740 & 0.104 \\
\hline 5 & 11411 & 2793 & 0.107 \\
\hline 7 & 15235 & 4650 & 0.134 \\
\hline 7 & 15124 & 4709 & 0.136 \\
\hline 7 & 18001 & 5582 & 0.136 \\
\hline 10 & 6450 & 2321 & 0.157 \\
\hline 10 & 6339 & 2348 & 0.162 \\
\hline 10 & 9476 & 3521 & 0.163 \\
\hline 15 & 7122 & 3469 & 0.213 \\
\hline 15 & 6708 & 3331 & 0.217 \\
\hline 15 & 6470 & 3289 & 0.222 \\
\hline 20 & 9267 & 5627 & 0.266 \\
\hline 20 & 10656 & 6415 & 0.263 \\
\hline 20 & 11835 & 7144 & 0.264 \\
\hline 30 & 9071 & 7183 & 0.346 \\
\hline 30 & 8575 & 6730 & 0.343 \\
\hline 30 & 10547 & 8529 & 0.354 \\
\hline 40 & 8816 & 8590 & 0.426 \\
\hline 40 & 8155 & 7882 & 0.423 \\
\hline 40 & 13793 & 13797 & 0.438 \\
\hline 50 & 7705 & 8756 & 0.497 \\
\hline 50 & 8747 & 10054 & 0.503 \\
\hline 50 & 11070 & 12909 & 0.510 \\
\hline 60 & 8052 & 10389 & 0.564 \\
\hline 60 & 8613 & 10996 & 0.559 \\
\hline 60 & 15614 & 20293 & 0.569 \\
\hline 75 & 10389 & 15521 & 0.654 \\
\hline 75 & 9028 & 13471 & 0.653 \\
\hline 75 & 12547 & 18871 & 0.658 \\
\hline 90 & 10485 & 17285 & 0.721 \\
\hline 90 & 8785 & 14353 & 0.715 \\
\hline 90 & 12371 & 20528 & 0.726 \\
\hline 120 & 12707 & 24060 & 0.828 \\
\hline 120 & 10201 & 19143 & 0.821 \\
\hline
\end{tabular}




\section{Order in $(E)$-1, (1.25 equiv).}

(E)-1

2-Iodothiophene

Tetra- $n$-butylammonium fluoride

Bis(dibenzylideneacetone)palladium $2.5 \mathrm{mM}$ (0.05 equiv)
$63 \mathrm{mM}$ (1.25 equiv)

$50 \mathrm{mM}$ (1.0 equiv)

$188 \mathrm{mM}$ (3.75 equiv)

Following the General Procedure, silanol (E)-1 (56 mg, $0.32 \mathrm{mmol})$ and biphenyl (7.3 $\mathrm{mg}$ ) were dissolved in TBAF solution $(940 \mu \mathrm{L}, 1.0 \mathrm{M}$ in THF, $0.94 \mathrm{mmol})$ in a flame dried 2neck round-bottomed flask, equipped with a stir bar and a septum under argon. Dry THF (3.05 $\mathrm{mL}$ ) was added and the clear, slightly-yellow solution was immersed into a water-bath at $23{ }^{\circ} \mathrm{C} \pm$ $1{ }^{\circ} \mathrm{C}$ for $30 \mathrm{~min}$. The 2-iodothiophene $(28 \mu \mathrm{L}, 0.25 \mathrm{mmol})$ was then added and the reaction was stirred for $1 \mathrm{~min}$. A suspension of $\operatorname{Pd}(\mathrm{dba})_{2}(7.2 \mathrm{mg}, 0.0125 \mathrm{mmol})$ in dry THF $(1.0 \mathrm{~mL})$ was added. The initially purple, turbid reaction mixture turns into a clear solution within 30 seconds. Aliquots $(\sim 200 \mu \mathrm{L})$ were withdrawn after 1, 2, 3, 4, 5, 7, 10, 15, 20, 30, 40, 50, 60, 75, 90, and $120 \mathrm{~min}$. To quench, these samples were added to $100 \mu \mathrm{L}$ of a $20 \%$ aqueous solution of 2dimethylaminoethanethiol hydrochloride. The clear colorless to slightly-yellow solution was then filtered through a plug of silica gel, and the silica gel was washed with diethyl ether to give a total sample volume of $\sim 2 \mathrm{~mL}$. This sample was injected into the GC three times.

initial rate $=0.0145$

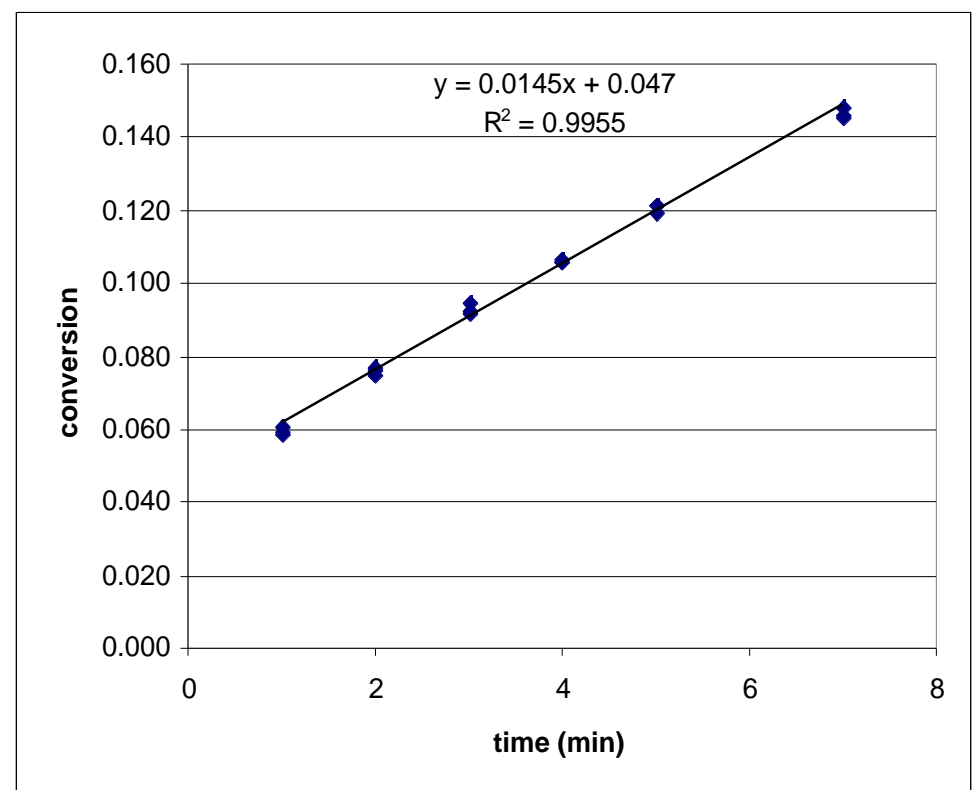




\begin{tabular}{|c|c|c|c|}
\hline time & area $\mathrm{A}$ & area 16 & conversion \\
\hline 1 & 8558 & 1809 & 0.058 \\
\hline 1 & 6570 & 1407 & 0.059 \\
\hline 1 & 8761 & 1932 & 0.061 \\
\hline 2 & 5296 & 1436 & 0.075 \\
\hline 2 & 7483 & 2064 & 0.076 \\
\hline 2 & 7995 & 2234 & 0.077 \\
\hline 3 & 7334 & 2458 & 0.092 \\
\hline 3 & 7834 & 2598 & 0.091 \\
\hline 3 & 9497 & 3261 & 0.095 \\
\hline 4 & 5401 & 2081 & 0.106 \\
\hline 4 & 6849 & 2641 & 0.106 \\
\hline 4 & 7762 & 2983 & 0.106 \\
\hline 5 & 6857 & 2965 & 0.119 \\
\hline 5 & 6635 & 2929 & 0.122 \\
\hline 5 & 8144 & 3587 & 0.121 \\
\hline 7 & 7727 & 4082 & 0.145 \\
\hline 7 & 8253 & 4428 & 0.148 \\
\hline 7 & 9903 & 5259 & 0.146 \\
\hline 10 & 6317 & 4105 & 0.179 \\
\hline 10 & 5710 & 3782 & 0.182 \\
\hline 10 & 4973 & 3301 & 0.183 \\
\hline 15 & 6048 & 5258 & 0.239 \\
\hline 15 & 7058 & 6095 & 0.238 \\
\hline 15 & 9432 & 8098 & 0.236 \\
\hline 20 & 7478 & 7664 & 0.282 \\
\hline 20 & 7080 & 7327 & 0.285 \\
\hline 20 & 9643 & 9957 & 0.284 \\
\hline 30 & 11497 & 15398 & 0.369 \\
\hline 30 & 12517 & 16428 & 0.361 \\
\hline 30 & 15197 & 20578 & 0.373 \\
\hline 40 & 16701 & 26478 & 0.436 \\
\hline 40 & 15133 & 24095 & 0.438 \\
\hline 40 & 16026 & 25440 & 0.437 \\
\hline 50 & 13452 & 24565 & 0.503 \\
\hline 50 & 14807 & 27093 & 0.504 \\
\hline 50 & 16001 & 29087 & 0.500 \\
\hline 60 & 8980 & 18136 & 0.556 \\
\hline 60 & 12796 & 25595 & 0.551 \\
\hline 60 & 11053 & 22319 & 0.556 \\
\hline 75 & 14064 & 31367 & 0.614 \\
\hline 75 & 13720 & 30672 & 0.615 \\
\hline 75 & 10970 & 24414 & 0.613 \\
\hline 90 & 8547 & 20790 & 0.670 \\
\hline 90 & 9282 & 22769 & 0.675 \\
\hline 90 & 12624 & 30670 & 0.669 \\
\hline 120 & 11538 & 31869 & 0.760 \\
\hline 120 & 13633 & 37292 & 0.753 \\
\hline
\end{tabular}


Order in (E)-1, (2.0 equiv).

(E)-1

2-Iodothiophene

Tetra- $n$-butylammonium fluoride

Bis(dibenzylideneacetone)palladium $2.5 \mathrm{mM}$ ( 0.05 equiv)
$100 \mathrm{mM}$ (2.0 equiv)

$50 \mathrm{mM}$ (1.0 equiv)

$188 \mathrm{mM}$ (3.75 equiv)

Following the General Procedure, silanol (E)-1 (86 mg, $0.50 \mathrm{mmol})$ and biphenyl (22.1 $\mathrm{mg}$ ) were dissolved in TBAF solution $(940 \mu \mathrm{L}, 1.0 \mathrm{M}$ in THF, $0.94 \mathrm{mmol})$ in a flame dried 2neck round-bottomed flask, equipped with a stir bar and a septum under argon. Dry THF (3.05 $\mathrm{mL}$ ) was added and the clear, slightly-yellow solution was immersed into a water-bath at $23{ }^{\circ} \mathrm{C} \pm$ $1{ }^{\circ} \mathrm{C}$ for $30 \mathrm{~min}$. The 2-iodothiophene $(28 \mu \mathrm{L}, 0.25 \mathrm{mmol})$ was then added and the reaction was stirred for $1 \mathrm{~min}$. A suspension of $\mathrm{Pd}(\mathrm{dba})_{2}(7.2 \mathrm{mg}, 0.0125 \mathrm{mmol})$ in dry THF $(1.0 \mathrm{~mL})$ was added. The initially purple, turbid reaction mixture turns into a clear solution within 30 seconds. Aliquots $(\sim 200 \mu \mathrm{L}$ )were withdrawn after 1, 2, 3, 4, 5, 7, 10, 15, 20, 30, 40, and $50 \mathrm{~min}$. To quench, these samples were added to $100 \mu \mathrm{L}$ of a $20 \%$ aqueous solution of 2dimethylaminoethanethiol hydrochloride. The clear colorless to slightly-yellow solution was then filtered through a plug of silica gel, and the silica gel was washed with diethyl ether to give a total sample volume of $\sim 2 \mathrm{~mL}$. This sample was injected into the GC three times.

initial rate $=0.0339$

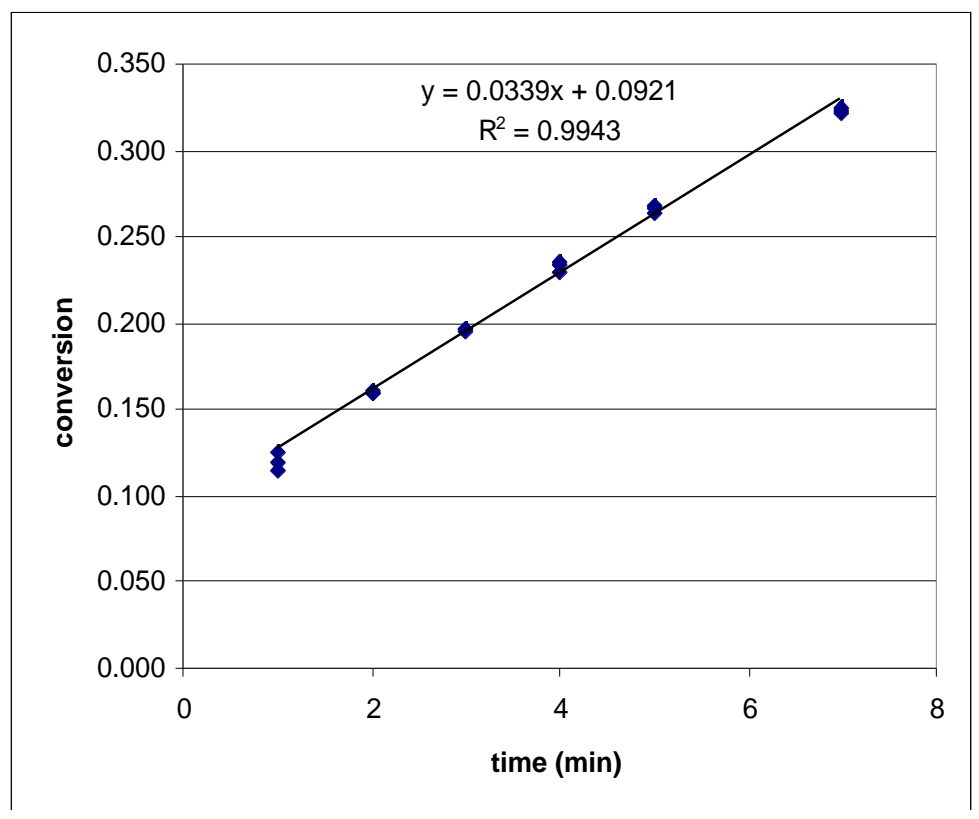




\begin{tabular}{|c|c|c|c|}
\hline time & $\operatorname{area} \mathrm{A}$ & area 16 & conversion \\
\hline 1 & 7999 & 1202 & 0.125 \\
\hline 1 & 8612 & 1192 & 0.115 \\
\hline 1 & 9837 & 1411 & 0.120 \\
\hline 2 & 7506 & 1440 & 0.160 \\
\hline 2 & 8136 & 1576 & 0.161 \\
\hline 2 & 9486 & 1815 & 0.159 \\
\hline 3 & 8601 & 2028 & 0.197 \\
\hline 3 & 9166 & 2150 & 0.195 \\
\hline 3 & 9870 & 2328 & 0.197 \\
\hline 4 & 8764 & 2476 & 0.235 \\
\hline 4 & 9594 & 2640 & 0.229 \\
\hline 4 & 9686 & 2720 & 0.234 \\
\hline 5 & 8316 & 2671 & 0.268 \\
\hline 5 & 9184 & 2903 & 0.263 \\
\hline 5 & 9459 & 3018 & 0.266 \\
\hline 7 & 8918 & 3445 & 0.322 \\
\hline 7 & 9634 & 3747 & 0.324 \\
\hline 7 & 10590 & 4106 & 0.323 \\
\hline 10 & 13020 & 6307 & 0.404 \\
\hline 10 & 14320 & 6932 & 0.403 \\
\hline 10 & 15202 & 7322 & 0.401 \\
\hline 15 & 10612 & 6591 & 0.518 \\
\hline 15 & 11321 & 6943 & 0.511 \\
\hline 15 & 11784 & 7248 & 0.513 \\
\hline 20 & 9675 & 7062 & 0.608 \\
\hline 20 & 9910 & 7105 & 0.598 \\
\hline 20 & 10508 & 7501 & 0.595 \\
\hline 30 & 12379 & 11030 & 0.743 \\
\hline 30 & 12652 & 11177 & 0.736 \\
\hline 30 & 13553 & 11950 & 0.735 \\
\hline 40 & 9304 & 9654 & 0.865 \\
\hline 40 & 9769 & 9901 & 0.845 \\
\hline 40 & 10265 & 10382 & 0.843 \\
\hline 50 & 10138 & 11592 & 0.953 \\
\hline 50 & 10182 & 11497 & 0.941 \\
\hline 50 & 11960 & 13528 & 0.943 \\
\hline
\end{tabular}


Order in (E)-1, (2.0 equiv).

(E)-1

2-Iodothiophene

Tetra- $n$-butylammonium fluoride

Bis(dibenzylideneacetone)palladium $2.5 \mathrm{mM}$ (0.05 equiv)
$100 \mathrm{mM}$ (2.0 equiv)

$50 \mathrm{mM}$ (1.0 equiv)

$188 \mathrm{mM}$ (3.75 equiv)

Following the General Procedure, silanol $(E)-1$ (86 mg, $0.50 \mathrm{mmol})$ and biphenyl (11.5 $\mathrm{mg}$ ) were dissolved in TBAF solution $(940 \mu \mathrm{L}, 1.0 \mathrm{M}$ in THF, $0.94 \mathrm{mmol})$ in a flame dried 2neck round-bottomed flask, equipped with a stir bar and a septum under argon. Dry THF (3.05 $\mathrm{mL}$ ) was added and the clear, slightly-yellow solution was immersed into a water-bath at $23{ }^{\circ} \mathrm{C} \pm$ $1{ }^{\circ} \mathrm{C}$ for $30 \mathrm{~min}$. The 2-iodothiophene $(28 \mu \mathrm{L}, 0.25 \mathrm{mmol})$ was then added and the reaction was stirred for $1 \mathrm{~min}$. A suspension of $\operatorname{Pd}(\mathrm{dba})_{2}(7.2 \mathrm{mg}, 0.0125 \mathrm{mmol})$ in dry THF $(1.0 \mathrm{~mL})$ was added. The initially purple, turbid reaction mixture turns into a clear solution within 30 seconds. Aliquots $(\sim 200 \mu \mathrm{L})$ were withdrawn after 1, 2, 3, 4, 5, 7, 10, 15, 20, 30, 40, 50, 60, 75, 90, and $120 \mathrm{~min}$. To quench, these samples were added to $100 \mu \mathrm{L}$ of a $20 \%$ aqueous solution of 2dimethylaminoethanethiol hydrochloride. The clear colorless to slightly-yellow solution was then filtered through a plug of silica gel, and the silica gel was washed with diethyl ether to give a total sample volume of $\sim 2 \mathrm{~mL}$. This sample was injected into the GC three times.

initial rate $=0.0336$

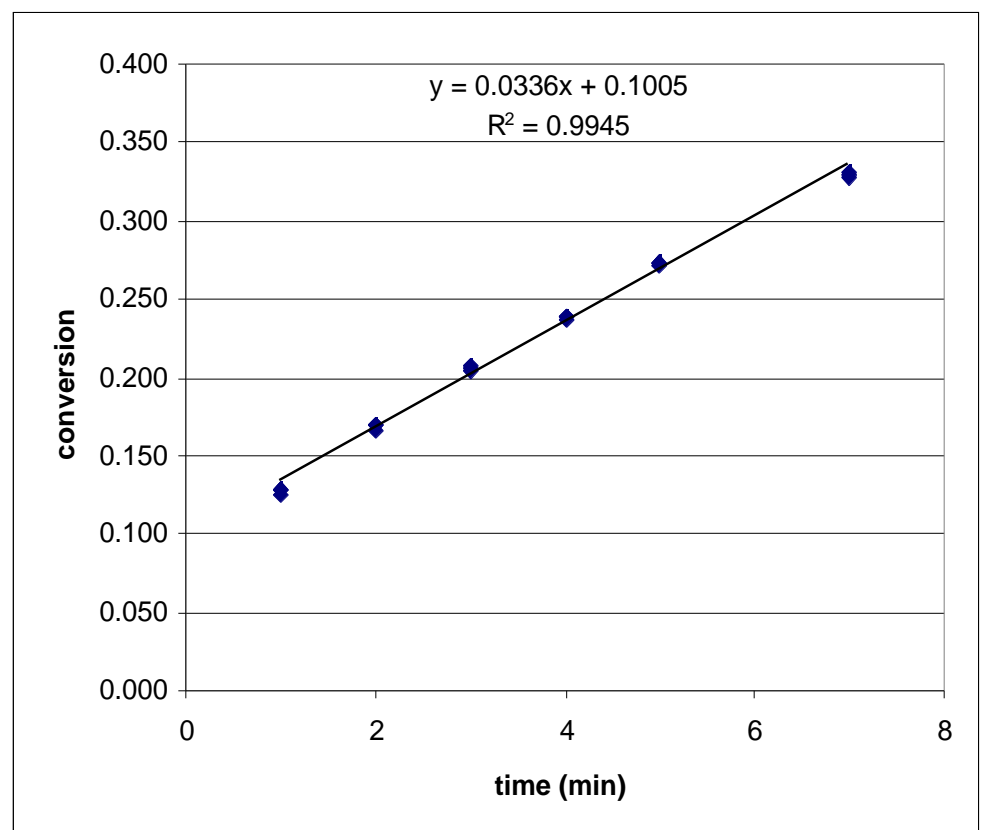




\begin{tabular}{|c|c|c|c|}
\hline time & area $\mathrm{A}$ & area 16 & conversion \\
\hline 1 & 7529 & 2179 & 0.126 \\
\hline 1 & 7012 & 2085 & 0.129 \\
\hline 1 & 7933 & 2336 & 0.128 \\
\hline 2 & 10000 & 3905 & 0.169 \\
\hline 2 & 6543 & 2548 & 0.169 \\
\hline 2 & 10062 & 3872 & 0.167 \\
\hline 3 & 10281 & 4888 & 0.206 \\
\hline 3 & 9175 & 4394 & 0.208 \\
\hline 3 & 13959 & 6557 & 0.204 \\
\hline 4 & 11847 & 6463 & 0.237 \\
\hline 4 & 9762 & 5361 & 0.238 \\
\hline 4 & 12077 & 6652 & 0.239 \\
\hline 5 & 6442 & 4069 & 0.274 \\
\hline 5 & 6817 & 4263 & 0.271 \\
\hline 5 & 8960 & 5643 & 0.273 \\
\hline 7 & 9787 & 7477 & 0.331 \\
\hline 7 & 6627 & 4993 & 0.327 \\
\hline 7 & 9251 & 7038 & 0.330 \\
\hline 10 & 7612 & 7232 & 0.412 \\
\hline 10 & 6692 & 6378 & 0.413 \\
\hline 10 & 10034 & 9601 & 0.415 \\
\hline 15 & 14807 & 18314 & 0.536 \\
\hline 15 & 10489 & 13005 & 0.538 \\
\hline 15 & 13235 & 16272 & 0.533 \\
\hline 20 & 12371 & 18140 & 0.636 \\
\hline 20 & 6406 & 9365 & 0.634 \\
\hline 20 & 10352 & 15155 & 0.635 \\
\hline 30 & 16136 & 30114 & 0.809 \\
\hline 30 & 10467 & 19472 & 0.807 \\
\hline 30 & 11016 & 20470 & 0.806 \\
\hline 40 & 17136 & 36764 & 0.930 \\
\hline 40 & 8881 & 19219 & 0.939 \\
\hline 40 & 16317 & 35171 & 0.935 \\
\hline 50 & 22832 & 54440 & 1.034 \\
\hline 50 & 14830 & 35708 & 1.044 \\
\hline 50 & 19521 & 46835 & 1.041 \\
\hline
\end{tabular}




\section{Order in (E)-1, (2.0 equiv).}

(E)-1

2-Iodothiophene

Tetra- $n$-butylammonium fluoride

Bis(dibenzylideneacetone)palladium $2.5 \mathrm{mM}$ (0.05 equiv)
$100 \mathrm{mM}$ (2.0 equiv)

$50 \mathrm{mM}$ (1.0 equiv)

$188 \mathrm{mM}$ (3.75 equiv)

Following the General Procedure, silanol (E)-1 (86 mg, $0.50 \mathrm{mmol})$ and biphenyl (12.4 $\mathrm{mg}$ ) were dissolved in TBAF solution $(940 \mu \mathrm{L}, 1.0 \mathrm{M}$ in THF, $0.94 \mathrm{mmol})$ in a flame dried 2neck round-bottomed flask, equipped with a stir bar and a septum under argon. Dry THF (3.05 $\mathrm{mL}$ ) was added and the clear, slightly-yellow solution was immersed into a water-bath at $23{ }^{\circ} \mathrm{C} \pm$ $1{ }^{\circ} \mathrm{C}$ for $30 \mathrm{~min}$. The 2-iodothiophene $(28 \mu \mathrm{L}, 0.25 \mathrm{mmol})$ was then added and the reaction was stirred for $1 \mathrm{~min}$. A suspension of $\operatorname{Pd}(\mathrm{dba})_{2}(7.2 \mathrm{mg}, 0.0125 \mathrm{mmol})$ in dry THF $(1.0 \mathrm{~mL})$ was added. The initially purple, turbid reaction mixture turns into a clear solution within 30 seconds. Aliquots $(\sim 200 \mu \mathrm{L})$ were withdrawn after 1, 2, 3, 4, 5, 7, 10, 15, 20, 30, 40, 50, and $60 \mathrm{~min}$. To quench, these samples were added to $100 \mu \mathrm{L}$ of a $20 \%$ aqueous solution of 2dimethylaminoethanethiol hydrochloride. The clear colorless to slightly-yellow solution was then filtered through a plug of silica gel, and the silica gel was washed with diethyl ether to give a total sample volume of $\sim 2 \mathrm{~mL}$. This sample was injected into the GC three times.

initial rate $=0.0283$

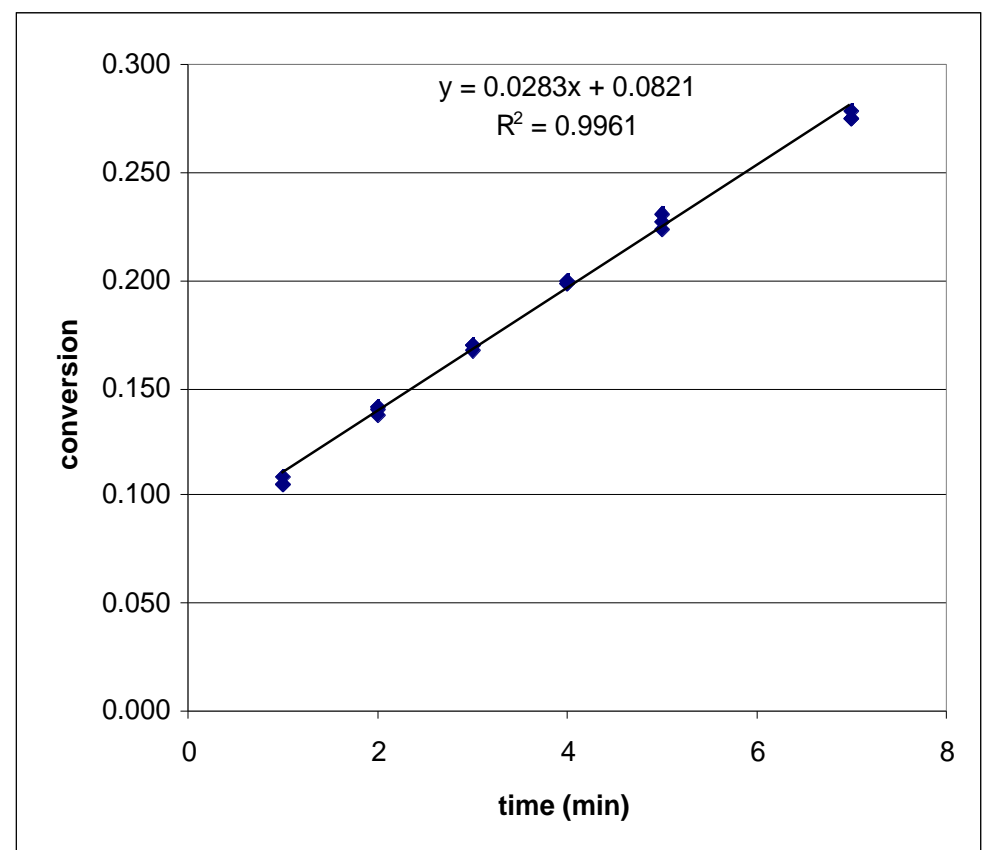




\begin{tabular}{|c|c|c|c|}
\hline time & area $\mathrm{A}$ & area $\mathbf{1 6}$ & conversion \\
\hline 1 & 8085 & 1874 & 0.108 \\
\hline 1 & 10213 & 2309 & 0.106 \\
\hline 1 & 12798 & 2889 & 0.106 \\
\hline 2 & 10794 & 3245 & 0.141 \\
\hline 2 & 13055 & 3891 & 0.139 \\
\hline 2 & 14578 & 4273 & 0.137 \\
\hline 3 & 14061 & 5087 & 0.169 \\
\hline 3 & 19594 & 7113 & 0.170 \\
\hline 3 & 23015 & 8219 & 0.167 \\
\hline 4 & 15791 & 6727 & 0.199 \\
\hline 4 & 27647 & 11755 & 0.199 \\
\hline 4 & 18995 & 8074 & 0.199 \\
\hline 5 & 10490 & 5023 & 0.224 \\
\hline 5 & 13292 & 6548 & 0.230 \\
\hline 5 & 17597 & 8529 & 0.227 \\
\hline 7 & 10573 & 6210 & 0.275 \\
\hline 7 & 14058 & 8373 & 0.279 \\
\hline 7 & 14719 & 8666 & 0.275 \\
\hline 10 & 8821 & 6575 & 0.349 \\
\hline 10 & 12373 & 9193 & 0.347 \\
\hline 10 & 13789 & 10227 & 0.347 \\
\hline 15 & 12090 & 11777 & 0.456 \\
\hline 15 & 19234 & 19077 & 0.464 \\
\hline 20 & 10025 & 11680 & 0.545 \\
\hline 20 & 17483 & 20222 & 0.541 \\
\hline 20 & 15959 & 18595 & 0.545 \\
\hline 30 & 11622 & 17269 & 0.695 \\
\hline 30 & 18793 & 27894 & 0.694 \\
\hline 30 & 19666 & 28776 & 0.684 \\
\hline 40 & 9122 & 15636 & 0.802 \\
\hline 40 & 13026 & 22343 & 0.802 \\
\hline 40 & 16788 & 28851 & 0.804 \\
\hline 50 & 9161 & 17612 & 0.899 \\
\hline 50 & 19648 & 37522 & 0.893 \\
\hline 50 & 22017 & 41920 & 0.890 \\
\hline 60 & 13803 & 28779 & 0.975 \\
\hline 60 & 20968 & 43385 & 0.968 \\
\hline 60 & 21611 & 44434 & 0.962 \\
\hline
\end{tabular}




\section{Order in $(E)-2,(0.5$ equiv $)$.}

(E)-2

2-Iodothiophene

Tetra-n-butylammonium fluoride

Bis(dibenzylideneacetone)palladium $6.25 \mathrm{mM}$ (0.05 equiv)
$62.5 \mathrm{mM}(0.5$ equiv $)$

$125 \mathrm{mM}$ (1.0 equiv)

$250 \mathrm{mM}$ (2.0 equiv)

Following the General Procedure, silanol (E)-2 (28.6 mg, 0.125 mmol) and biphenyl (7.5 $\mathrm{mg}$ ) were dissolved in TBAF solution (500 $\mu \mathrm{L}, 1.0 \mathrm{M}$ in THF, $0.5 \mathrm{mmol})$ in a flame dried 2-neck round-bottomed flask, equipped with a stir bar and a septum under argon. Dry THF $(0.7 \mathrm{~mL})$ was added and the clear, slightly-yellow solution was immersed into a water-bath at $23{ }^{\circ} \mathrm{C} \pm 1$ ${ }^{\circ} \mathrm{C}$ for $30 \mathrm{~min}$. The 2-iodothiophene $(28 \mu \mathrm{L}, 0.25 \mathrm{mmol})$ was then added and the reaction was stirred for $1 \mathrm{~min}$. A suspension of $\operatorname{Pd}(\mathrm{dba})_{2}(7.2 \mathrm{mg}, 0.0125 \mathrm{mmol})$ in dry THF $(1.0 \mathrm{~mL})$ was added. The initially purple, turbid reaction mixture turns into a clear solution within 30 seconds. Aliquots ( $200 \mathrm{uL} \_$were withdrawn after 1, 2, 3, 4, 5, 7, 10, 15, 20, 30, 40, 50, 60, 75, 90, and $120 \mathrm{~min}$. To quench, these samples were added to $100 \mathrm{uL}$ of a $20 \%$ aqueous solution of 2dimethylaminoethanethiol hydrochloride. The clear colorless to slightly-yellow solution was then filtered through a plug of silica gel, and the silica gel was washed with diethyl ether to give a total sample volume of $\sim 2 \mathrm{~mL}$. This sample was injected into the $\mathrm{GC}$ three times.

initial rate $=0.0022$

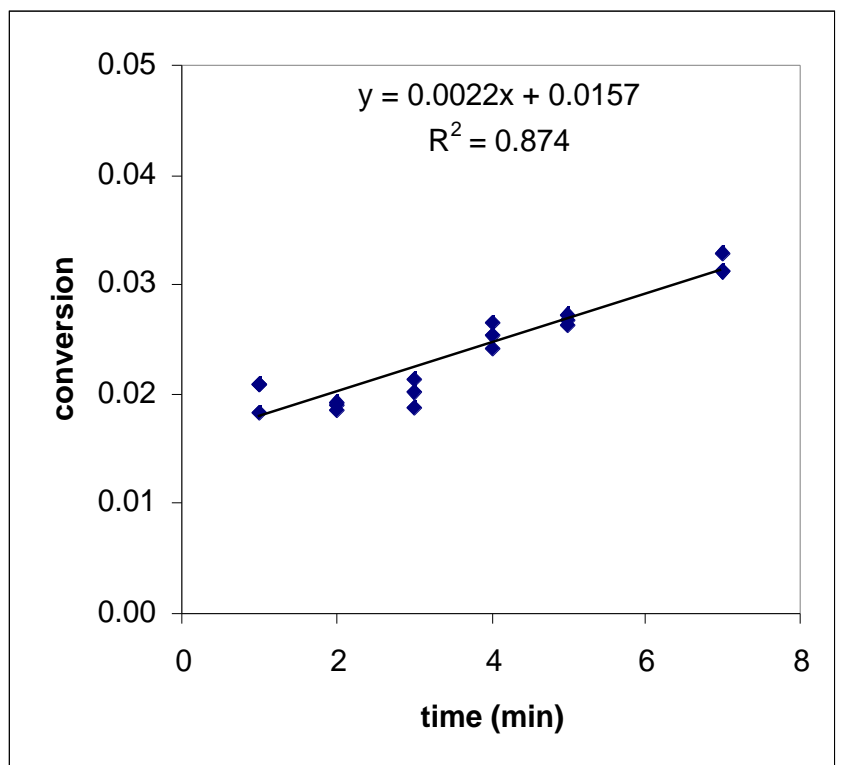




\begin{tabular}{|c|c|c|c|}
\hline time (min) & area $\mathrm{A}$ & area 16 & conversion \\
\hline 1 & 2177 & 161 & 0.021 \\
\hline 1 & 2202 & 142 & 0.018 \\
\hline 1 & 2157 & 160 & 0.021 \\
\hline 2 & 12436 & 814 & 0.019 \\
\hline 2 & 12392 & 832 & 0.019 \\
\hline 2 & 12425 & 845 & 0.019 \\
\hline 3 & 10953 & 826 & 0.021 \\
\hline 3 & 10958 & 785 & 0.020 \\
\hline 3 & 10999 & 727 & 0.019 \\
\hline 4 & 11646 & 998 & 0.024 \\
\hline 4 & 11541 & 1080 & 0.026 \\
\hline 4 & 11706 & 1046 & 0.025 \\
\hline 5 & 9787 & 930 & 0.027 \\
\hline 5 & 9761 & 907 & 0.026 \\
\hline 5 & 9634 & 927 & 0.027 \\
\hline 7 & 11376 & 1323 & 0.033 \\
\hline 7 & 11351 & 1251 & 0.031 \\
\hline 7 & 11217 & 1238 & 0.031 \\
\hline 10 & 7792 & 997 & 0.036 \\
\hline 10 & 7717 & 1041 & 0.038 \\
\hline 10 & 7743 & 991 & 0.036 \\
\hline 15 & 9979 & 1660 & 0.047 \\
\hline 15 & 10098 & 1662 & 0.047 \\
\hline 15 & 10101 & 1632 & 0.046 \\
\hline 20 & 11783 & 2185 & 0.052 \\
\hline 20 & 11801 & 2218 & 0.053 \\
\hline 20 & 11742 & 2156 & 0.052 \\
\hline 30 & 8638 & 2044 & 0.067 \\
\hline 30 & 8689 & 2112 & 0.069 \\
\hline 30 & 8672 & 2068 & 0.067 \\
\hline 40 & 11665 & 3254 & 0.079 \\
\hline 40 & 11724 & 3209 & 0.077 \\
\hline 40 & 11612 & 3211 & 0.078 \\
\hline 50 & 9225 & 3048 & 0.093 \\
\hline 50 & 9204 & 2910 & 0.089 \\
\hline 50 & 9218 & 2945 & 0.090 \\
\hline 60 & 7480 & 2694 & 0.102 \\
\hline 60 & 7397 & 2739 & 0.105 \\
\hline 60 & 7404 & 2697 & 0.103 \\
\hline 75 & 7989 & 3433 & 0.122 \\
\hline 75 & 8030 & 3450 & 0.122 \\
\hline 75 & 8047 & 3380 & 0.119 \\
\hline 90 & 5407 & 2741 & 0.143 \\
\hline 90 & 5411 & 2495 & 0.130 \\
\hline 90 & 5397 & 2601 & 0.136 \\
\hline 120 & 7939 & 4719 & 0.168 \\
\hline 120 & 8064 & 4800 & 0.168 \\
\hline 120 & 7918 & 4566 & 0.163 \\
\hline
\end{tabular}




\section{Order in $(E)-2,(0.5$ equiv $)$.}

(E)-2

2-Iodothiophene

Tetra-n-butylammonium fluoride

Bis(dibenzylideneacetone)palladium $6.25 \mathrm{mM}$ (0.05 equiv)
$62.5 \mathrm{mM}(0.5$ equiv $)$

$125 \mathrm{mM}$ (1.0 equiv)

$250 \mathrm{mM}$ (2.0 equiv)

Following the General Procedure, silanol (E)-2 (28.6 mg, $0.125 \mathrm{mmol})$ and biphenyl (8.8 $\mathrm{mg}$ ) were dissolved in TBAF solution $(500 \mu \mathrm{L}, 1.0 \mathrm{M}$ in THF, $0.5 \mathrm{mmol})$ in a flame dried 2-neck round-bottomed flask, equipped with a stir bar and a septum under argon. Dry THF $(0.7 \mathrm{~mL})$ was added and the clear, slightly-yellow solution was immersed into a water-bath at $23{ }^{\circ} \mathrm{C} \pm 1$ ${ }^{\circ} \mathrm{C}$ for $30 \mathrm{~min}$. The 2-iodothiophene $(28 \mu \mathrm{L}, 0.25 \mathrm{mmol})$ was then added and the reaction was stirred for $1 \mathrm{~min}$. A suspension of $\operatorname{Pd}(\mathrm{dba})_{2}(7.2 \mathrm{mg}, 0.0125 \mathrm{mmol})$ in dry THF $(1.0 \mathrm{~mL})$ was added. The initially purple, turbid reaction mixture turns into a clear solution within 30 seconds. Aliquots ( $200 \mathrm{uL} \_$were withdrawn after 1, 2, 3, 4, 5, 7, 10, 15, 20, 30, 40, 50, 60, 75, 90, and $120 \mathrm{~min}$. To quench, these samples were added to $100 \mathrm{uL}$ of a $20 \%$ aqueous solution of 2dimethylaminoethanethiol hydrochloride. The clear colorless to slightly-yellow solution was then filtered through a plug of silica gel, and the silica gel was washed with diethyl ether to give a total sample volume of $\sim 2 \mathrm{~mL}$. This sample was injected into the GC three times.

initial rate $=0.0033$

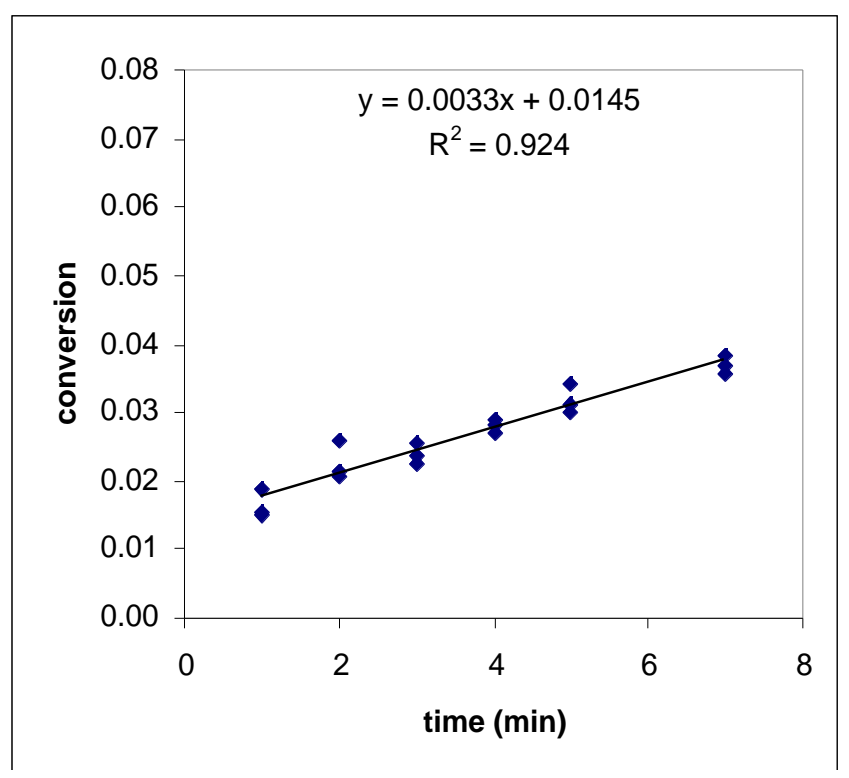




\begin{tabular}{|c|c|c|c|}
\hline time (min) & area $\mathrm{A}$ & area 16 & conversion \\
\hline 1 & 7416 & 423 & 0.019 \\
\hline 1 & 7761 & 358 & 0.015 \\
\hline 1 & 7821 & 355 & 0.015 \\
\hline 2 & 8565 & 531 & 0.021 \\
\hline 2 & 8599 & 558 & 0.022 \\
\hline 2 & 8643 & 679 & 0.026 \\
\hline 3 & 6931 & 470 & 0.023 \\
\hline 3 & 6955 & 534 & 0.025 \\
\hline 3 & 6921 & 495 & 0.024 \\
\hline 4 & 8142 & 711 & 0.029 \\
\hline 4 & 8103 & 684 & 0.028 \\
\hline 4 & 7995 & 647 & 0.027 \\
\hline 5 & 8221 & 771 & 0.031 \\
\hline 5 & 8267 & 748 & 0.030 \\
\hline 5 & 8225 & 843 & 0.034 \\
\hline 7 & 9046 & 973 & 0.036 \\
\hline 7 & 8983 & 994 & 0.037 \\
\hline 7 & 8969 & 1037 & 0.038 \\
\hline 10 & 9745 & 1185 & 0.040 \\
\hline 10 & 9697 & 1142 & 0.039 \\
\hline 10 & 9702 & 1193 & 0.041 \\
\hline 15 & 5973 & 922 & 0.051 \\
\hline 15 & 5920 & 863 & 0.048 \\
\hline 15 & 5915 & 869 & 0.049 \\
\hline 20 & 7904 & 1524 & 0.064 \\
\hline 20 & 7972 & 1287 & 0.054 \\
\hline 20 & 7994 & 1361 & 0.057 \\
\hline 30 & 9456 & 2024 & 0.071 \\
\hline 30 & 9403 & 1859 & 0.066 \\
\hline 30 & 9400 & 1979 & 0.070 \\
\hline 40 & 9112 & 2111 & 0.077 \\
\hline 40 & 9051 & 2293 & 0.084 \\
\hline 40 & 9113 & 2197 & 0.080 \\
\hline 50 & 11646 & 3001 & 0.086 \\
\hline 50 & 11834 & 2949 & 0.083 \\
\hline 50 & 11832 & 3068 & 0.086 \\
\hline 60 & 8625 & 2388 & 0.092 \\
\hline 60 & 8520 & 2475 & 0.096 \\
\hline 60 & 8532 & 2509 & 0.098 \\
\hline 75 & 5811 & 1979 & 0.113 \\
\hline 75 & 5743 & 1909 & 0.110 \\
\hline 75 & 5798 & 2000 & 0.114 \\
\hline 90 & 7475 & 2916 & 0.129 \\
\hline 90 & 7471 & 2899 & 0.129 \\
\hline 90 & 7434 & 2743 & 0.122 \\
\hline 120 & 6673 & 2873 & 0.143 \\
\hline 120 & 6618 & 3028 & 0.152 \\
\hline 120 & 6654 & 2944 & 0.147 \\
\hline
\end{tabular}




\section{Order in $(E)-2,(0.5$ equiv $)$.}

(E)-2

2-Iodothiophene

Tetra-n-butylammonium fluoride

Bis(dibenzylideneacetone)palladium $6.25 \mathrm{mM}$ (0.05 equiv)
$62.5 \mathrm{mM}(0.5$ equiv $)$

$125 \mathrm{mM}$ (1.0 equiv)

$250 \mathrm{mM}$ (2.0 equiv)

Following the General Procedure, silanol (E)-2 $(28.6 \mathrm{mg}, 0.125 \mathrm{mmol})$ and biphenyl (11.1 mg) were dissolved in TBAF solution $(500 \mu \mathrm{L}, 1.0 \mathrm{M}$ in THF, $0.5 \mathrm{mmol})$ in a flame dried 2-neck round-bottomed flask, equipped with a stir bar and a septum under argon. Dry THF (0.7 $\mathrm{mL}$ ) was added and the clear, slightly-yellow solution was immersed into a water-bath at $23{ }^{\circ} \mathrm{C} \pm$ $1{ }^{\circ} \mathrm{C}$ for $30 \mathrm{~min}$. The 2-iodothiophene $(28 \mu \mathrm{L}, 0.25 \mathrm{mmol})$ was then added and the reaction was stirred for $1 \mathrm{~min}$. A suspension of $\operatorname{Pd}(\mathrm{dba})_{2}(7.2 \mathrm{mg}, 0.0125 \mathrm{mmol})$ in dry THF $(1.0 \mathrm{~mL})$ was added. The initially purple, turbid reaction mixture turns into a clear solution within 30 seconds. Aliquots $\left(\sim 200 \mathrm{uL} \_\right.$were withdrawn after 1, 2, 3, 4, 5, 7, 10, 15, 20, 30, 40, 50, 60, 75, 90, and $120 \mathrm{~min}$. To quench, these samples were added to $100 \mathrm{uL}$ of a $20 \%$ aqueous solution of 2dimethylaminoethanethiol hydrochloride. The clear colorless to slightly-yellow solution was then filtered through a plug of silica gel, and the silica gel was washed with diethyl ether to give a total sample volume of $\sim 2 \mathrm{~mL}$. This sample was injected into the $\mathrm{GC}$ three times.

initial rate $=0.0031$

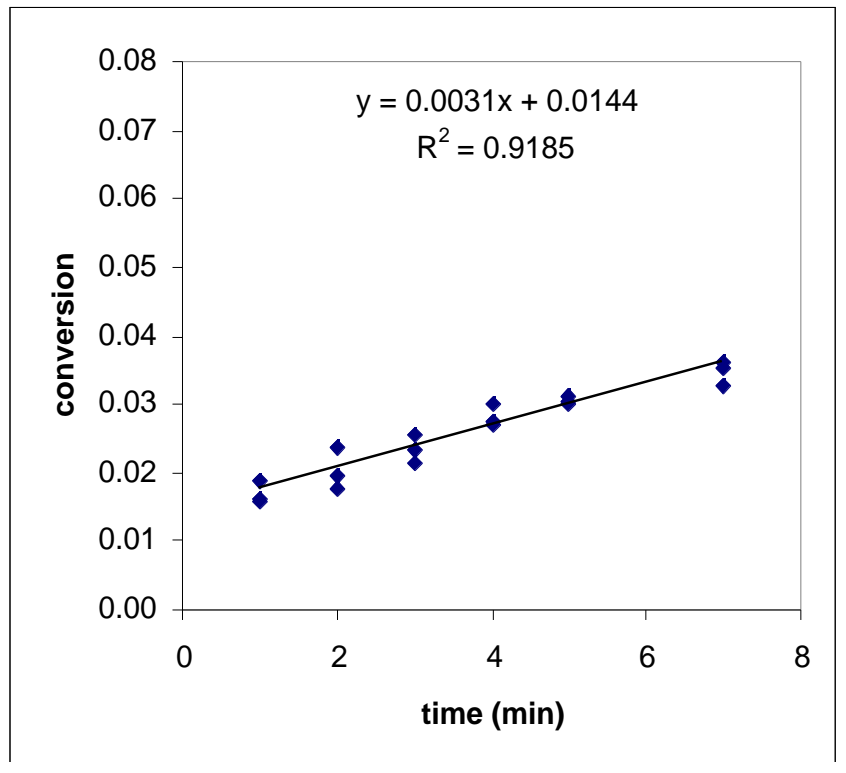




\begin{tabular}{|c|c|c|c|}
\hline time (min) & $\operatorname{area} \mathrm{A}$ & area 16 & conversion \\
\hline 1 & 12345 & 557 & 0.019 \\
\hline 1 & 12281 & 469 & 0.016 \\
\hline 1 & 12449 & 464 & 0.016 \\
\hline 2 & 11776 & 548 & 0.019 \\
\hline 2 & 11846 & 503 & 0.018 \\
\hline 2 & 11725 & 659 & 0.024 \\
\hline 3 & 11810 & 606 & 0.021 \\
\hline 3 & 11853 & 664 & 0.023 \\
\hline 3 & 11745 & 715 & 0.025 \\
\hline 4 & 10951 & 783 & 0.030 \\
\hline 4 & 11086 & 716 & 0.027 \\
\hline 4 & 10960 & 720 & 0.028 \\
\hline 5 & 8631 & 624 & 0.030 \\
\hline 5 & 8559 & 617 & 0.030 \\
\hline 5 & 8520 & 638 & 0.031 \\
\hline 7 & 11115 & 867 & 0.033 \\
\hline 7 & 11014 & 953 & 0.036 \\
\hline 7 & 11165 & 943 & 0.035 \\
\hline 10 & 8602 & 916 & 0.045 \\
\hline 10 & 9032 & 1120 & 0.052 \\
\hline 10 & 8904 & 1200 & 0.056 \\
\hline 15 & 11515 & 1418 & 0.052 \\
\hline 15 & 11470 & 1333 & 0.049 \\
\hline 15 & 11475 & 1387 & 0.051 \\
\hline 20 & 13116 & 2084 & 0.067 \\
\hline 20 & 13322 & 1782 & 0.056 \\
\hline 20 & 13283 & 1838 & 0.058 \\
\hline 30 & 11797 & 1948 & 0.069 \\
\hline 30 & 11713 & 2010 & 0.072 \\
\hline 30 & 11727 & 1954 & 0.070 \\
\hline 40 & 13428 & 2485 & 0.077 \\
\hline 40 & 13233 & 2619 & 0.083 \\
\hline 40 & 13251 & 2506 & 0.079 \\
\hline 50 & 8869 & 2004 & 0.095 \\
\hline 50 & 8690 & 1889 & 0.091 \\
\hline 50 & 8774 & 1952 & 0.093 \\
\hline 60 & 10872 & 2520 & 0.097 \\
\hline 60 & 10870 & 2621 & 0.101 \\
\hline 60 & 10809 & 2851 & 0.110 \\
\hline 75 & 12507 & 3571 & 0.120 \\
\hline 75 & 12379 & 3359 & 0.114 \\
\hline 75 & 12489 & 3292 & 0.110 \\
\hline 90 & 9529 & 2866 & 0.126 \\
\hline 90 & 9497 & 2960 & 0.130 \\
\hline 90 & 9541 & 2837 & 0.124 \\
\hline 120 & 17658 & 6556 & 0.155 \\
\hline 120 & 17932 & 6476 & 0.151 \\
\hline 120 & 18038 & 6700 & 0.155 \\
\hline
\end{tabular}


Order in $(E)-2,(0.75$ equiv $)$.

(E)-2

2-Iodothiophene

Tetra-n-butylammonium fluoride

Bis(dibenzylideneacetone)palladium $6.25 \mathrm{mM}$ (0.05 equiv)
$93.8 \mathrm{mM}(0.75$ equiv $)$

$125 \mathrm{mM}$ (1.0 equiv)

$250 \mathrm{mM}$ (2.0 equiv)

Following the General Procedure, silanol (E)-2 (42.8 mg, $0.188 \mathrm{mmol})$ and biphenyl $(8.2$ $\mathrm{mg})$ were dissolved in TBAF solution $((500 \mu \mathrm{L}, 1.0 \mathrm{M}$ in THF, $0.5 \mathrm{mmol})$ in a flame dried 2neck round-bottomed flask, equipped with a stir bar and a septum under argon. Dry THF (0.7 $\mathrm{mL}$ ) was added and the clear, slightly-yellow solution was immersed into a water-bath at $23{ }^{\circ} \mathrm{C} \pm$ $1{ }^{\circ} \mathrm{C}$ for $30 \mathrm{~min}$. The 2-iodothiophene $(28 \mu \mathrm{L}, 0.25 \mathrm{mmol})$ was then added and the reaction was stirred for $1 \mathrm{~min}$. A suspension of $\operatorname{Pd}(\mathrm{dba})_{2}(7.2 \mathrm{mg}, 0.0125 \mathrm{mmol})$ in dry THF $(1.0 \mathrm{~mL})$ was added. The initially purple, turbid reaction mixture turns into a clear solution within 30 seconds. Aliquots $(\sim 200 \mu \mathrm{L})$ were withdrawn after 1, 2, 3, 4, 5, 7, 10, 15, 20, 30, 40, 50, 60, 75, 90, and $120 \mathrm{~min}$. To quench, these samples were added to $100 \mu \mathrm{L}$ of a $20 \%$ aqueous solution of 2dimethylaminoethanethiol hydrochloride. The clear colorless to slightly-yellow solution was then filtered through a plug of silica gel, and the silica gel was washed with diethyl ether to give a total sample volume of $\sim 2 \mathrm{~mL}$. This sample was injected into the GC three times.

initial rate $=0.0074$

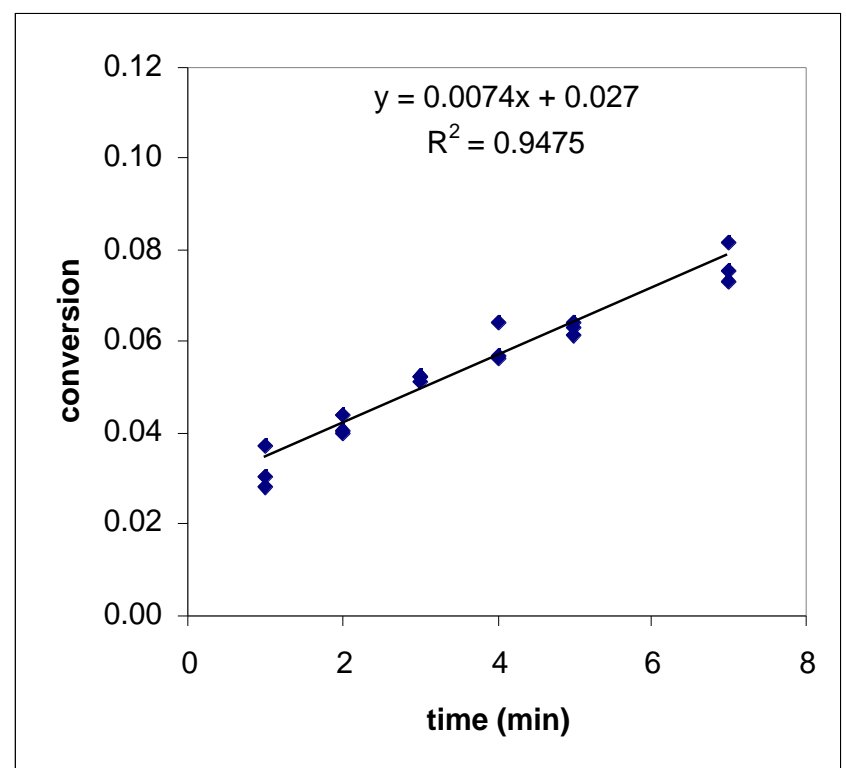




\begin{tabular}{|c|c|c|c|}
\hline time $(\min )$ & area $\mathrm{A}$ & area 16 & conversion \\
\hline 1 & 8295 & 607 & 0.030 \\
\hline 1 & 8505 & 763 & 0.037 \\
\hline 1 & 8267 & 561 & 0.028 \\
\hline 2 & 8831 & 871 & 0.041 \\
\hline 2 & 8973 & 951 & 0.044 \\
\hline 2 & 8993 & 867 & 0.040 \\
\hline 3 & 7779 & 985 & 0.052 \\
\hline 3 & 7848 & 994 & 0.052 \\
\hline 3 & 7698 & 962 & 0.052 \\
\hline 4 & 7203 & 998 & 0.057 \\
\hline 4 & 7094 & 1105 & 0.064 \\
\hline 4 & 7097 & 974 & 0.057 \\
\hline 5 & 8400 & 1309 & 0.064 \\
\hline 5 & 8444 & 1260 & 0.062 \\
\hline 5 & 8394 & 1285 & 0.063 \\
\hline 7 & 7442 & 1326 & 0.073 \\
\hline 7 & 7564 & 1388 & 0.076 \\
\hline 7 & 7404 & 1470 & 0.082 \\
\hline 10 & 8934 & 2037 & 0.094 \\
\hline 10 & 8879 & 2089 & 0.097 \\
\hline 10 & 8898 & 1957 & 0.091 \\
\hline 15 & 7962 & 2242 & 0.116 \\
\hline 15 & 7994 & 2251 & 0.116 \\
\hline 15 & 8015 & 2162 & 0.111 \\
\hline 20 & 9391 & 2957 & 0.130 \\
\hline 20 & 9355 & 2983 & 0.131 \\
\hline 20 & 9348 & 3109 & 0.137 \\
\hline 30 & 12594 & 4822 & 0.158 \\
\hline 30 & 12436 & 5004 & 0.166 \\
\hline 30 & 12485 & 4755 & 0.157 \\
\hline 40 & 10685 & 4974 & 0.192 \\
\hline 40 & 10558 & 5170 & 0.202 \\
\hline 40 & 10664 & 5069 & 0.196 \\
\hline 50 & 11520 & 6241 & 0.223 \\
\hline 50 & 11460 & 6312 & 0.227 \\
\hline 50 & 11541 & 6253 & 0.223 \\
\hline 60 & 11683 & 7280 & 0.257 \\
\hline 60 & 11698 & 7255 & 0.256 \\
\hline 60 & 11704 & 7282 & 0.257 \\
\hline 75 & 8479 & 6412 & 0.312 \\
\hline 75 & 8417 & 6366 & 0.312 \\
\hline 75 & 8401 & 6394 & 0.314 \\
\hline 90 & 9945 & 8371 & 0.347 \\
\hline 90 & 9906 & 8453 & 0.352 \\
\hline 90 & 10011 & 8453 & 0.348 \\
\hline 120 & 10449 & 11524 & 0.455 \\
\hline 120 & 10485 & 11600 & 0.456 \\
\hline 120 & 10519 & 11599 & 0.455 \\
\hline
\end{tabular}


Order in $(E)-2,(0.75$ equiv $)$.

(E)-2

2-Iodothiophene

Tetra-n-butylammonium fluoride

Bis(dibenzylideneacetone)palladium $6.25 \mathrm{mM}$ (0.05 equiv)
$93.8 \mathrm{mM}(0.75$ equiv $)$

$125 \mathrm{mM}$ (1.0 equiv)

$250 \mathrm{mM}$ (2.0 equiv)

Following the General Procedure, silanol $(E)-2(42.8 \mathrm{mg}, 0.188 \mathrm{mmol})$ and biphenyl $(8.7$ $\mathrm{mg})$ were dissolved in TBAF solution $((500 \mu \mathrm{L}, 1.0 \mathrm{M}$ in THF, $0.5 \mathrm{mmol})$ in a flame dried 2neck round-bottomed flask, equipped with a stir bar and a septum under argon. Dry THF (0.7 $\mathrm{mL}$ ) was added and the clear, slightly-yellow solution was immersed into a water-bath at $23{ }^{\circ} \mathrm{C} \pm$ $1{ }^{\circ} \mathrm{C}$ for $30 \mathrm{~min}$. The 2-iodothiophene $(28 \mu \mathrm{L}, 0.25 \mathrm{mmol})$ was then added and the reaction was stirred for $1 \mathrm{~min}$. A suspension of $\operatorname{Pd}(\mathrm{dba})_{2}(7.2 \mathrm{mg}, 0.0125 \mathrm{mmol})$ in dry THF $(1.0 \mathrm{~mL})$ was added. The initially purple, turbid reaction mixture turns into a clear solution within 30 seconds. Aliquots $(\sim 200 \mu \mathrm{L})$ were withdrawn after 1, 2, 3, 4, 5, 7, 10, 15, 20, 30, 40, 50, 60, 75, 90, and $120 \mathrm{~min}$. To quench, these samples were added to $100 \mu \mathrm{L}$ of a $20 \%$ aqueous solution of 2dimethylaminoethanethiol hydrochloride. The clear colorless to slightly-yellow solution was then filtered through a plug of silica gel, and the silica gel was washed with diethyl ether to give a total sample volume of $\sim 2 \mathrm{~mL}$. This sample was injected into the GC three times.

initial rate $=0.0058$

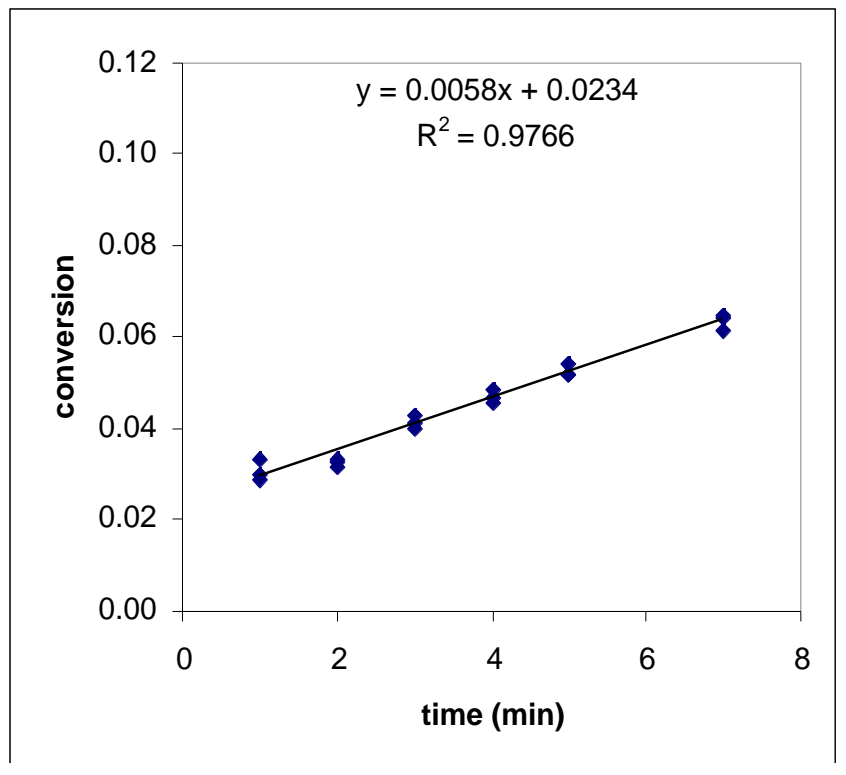




\begin{tabular}{|c|c|c|c|}
\hline time $(\min )$ & area $\mathrm{A}$ & area 16 & conversion \\
\hline 1 & 11453 & 781 & 0.030 \\
\hline 1 & 11557 & 877 & 0.033 \\
\hline 1 & 11511 & 763 & 0.029 \\
\hline 2 & 8520 & 635 & 0.033 \\
\hline 2 & 8422 & 603 & 0.031 \\
\hline 2 & 8460 & 640 & 0.033 \\
\hline 3 & 8440 & 769 & 0.040 \\
\hline 3 & 8249 & 803 & 0.043 \\
\hline 3 & 8122 & 762 & 0.041 \\
\hline 4 & 8694 & 959 & 0.048 \\
\hline 4 & 8480 & 903 & 0.047 \\
\hline 4 & 8460 & 887 & 0.046 \\
\hline 5 & 8975 & 1064 & 0.052 \\
\hline 5 & 8921 & 1104 & 0.054 \\
\hline 5 & 8842 & 1051 & 0.052 \\
\hline 7 & 9080 & 1343 & 0.065 \\
\hline 7 & 9102 & 1338 & 0.064 \\
\hline 7 & 9184 & 1295 & 0.062 \\
\hline 10 & 9953 & 1857 & 0.082 \\
\hline 10 & 9907 & 1825 & 0.081 \\
\hline 10 & 9990 & 1810 & 0.079 \\
\hline 15 & 9560 & 2178 & 0.100 \\
\hline 15 & 9525 & 2156 & 0.099 \\
\hline 15 & 9597 & 2123 & 0.097 \\
\hline 20 & 10518 & 2797 & 0.116 \\
\hline 20 & 10425 & 2788 & 0.117 \\
\hline 20 & 10392 & 2793 & 0.118 \\
\hline 30 & 9189 & 3106 & 0.148 \\
\hline 30 & 9180 & 3103 & 0.148 \\
\hline 30 & 9180 & 3131 & 0.149 \\
\hline 40 & 10490 & 4192 & 0.175 \\
\hline 40 & 10408 & 4195 & 0.176 \\
\hline 40 & 10473 & 4242 & 0.177 \\
\hline 50 & 9370 & 4462 & 0.208 \\
\hline 50 & 9315 & 4406 & 0.207 \\
\hline 50 & 9324 & 4402 & 0.207 \\
\hline 60 & 6674 & 3585 & 0.235 \\
\hline 60 & 6696 & 3560 & 0.233 \\
\hline 60 & 6733 & 3691 & 0.240 \\
\hline 75 & 6245 & 4020 & 0.282 \\
\hline 75 & 6236 & 3957 & 0.278 \\
\hline 75 & 6321 & 4098 & 0.284 \\
\hline 90 & 5808 & 4231 & 0.319 \\
\hline 90 & 5880 & 4202 & 0.313 \\
\hline 90 & 5949 & 4279 & 0.315 \\
\hline 120 & 8711 & 7667 & 0.385 \\
\hline 120 & 8614 & 7620 & 0.387 \\
\hline 120 & 8747 & 7746 & 0.387 \\
\hline
\end{tabular}


Order in $(E)-2,(0.75$ equiv).

(E)-2

2-Iodothiophene

Tetra-n-butylammonium fluoride

Bis(dibenzylideneacetone)palladium $6.25 \mathrm{mM}$ (0.05 equiv)
$93.8 \mathrm{mM}$ ( 0.75 equiv)

$250 \mathrm{mM}$ (2.0 equiv)
$125 \mathrm{mM}$ (1.0 equiv)

Following the General Procedure, silanol (E)-2 (42.8 mg, $0.188 \mathrm{mmol})$ and biphenyl (6.3 $\mathrm{mg})$ were dissolved in TBAF solution $((500 \mu \mathrm{L}, 1.0 \mathrm{M}$ in THF, $0.5 \mathrm{mmol})$ in a flame dried 2neck round-bottomed flask, equipped with a stir bar and a septum under argon. Dry THF (0.7 $\mathrm{mL}$ ) was added and the clear, slightly-yellow solution was immersed into a water-bath at $23{ }^{\circ} \mathrm{C} \pm$ $1{ }^{\circ} \mathrm{C}$ for $30 \mathrm{~min}$. The 2-iodothiophene $(28 \mu \mathrm{L}, 0.25 \mathrm{mmol})$ was then added and the reaction

$\mathrm{n}$ was stirred for $1 \mathrm{~min}$. A suspension of $\mathrm{Pd}(\mathrm{dba})_{2}(7.2 \mathrm{mg}, 0.0125 \mathrm{mmol})$ in dry THF $(1.0 \mathrm{~mL})$ was added. The initially purple, turbid reaction mixture turns into a clear solution within 30 seconds. Aliquots $(\sim 200 \mu \mathrm{L})$ were withdrawn after 1, 2, 3, 4, 5, 7, 10, 15, 20, 30, 40, $50,60,75,90$, and $120 \mathrm{~min}$. To quench, these samples were added to $100 \mu \mathrm{L}$ of a $20 \%$ aqueous solution of 2-dimethylaminoethanethiol hydrochloride. The clear colorless to slightly-yellow solution was then filtered through a plug of silica gel, and the silica gel was washed with diethyl ether to give a total sample volume of $\sim 2 \mathrm{~mL}$. This sample was injected into the GC three times.

initial rate $=0.0077$

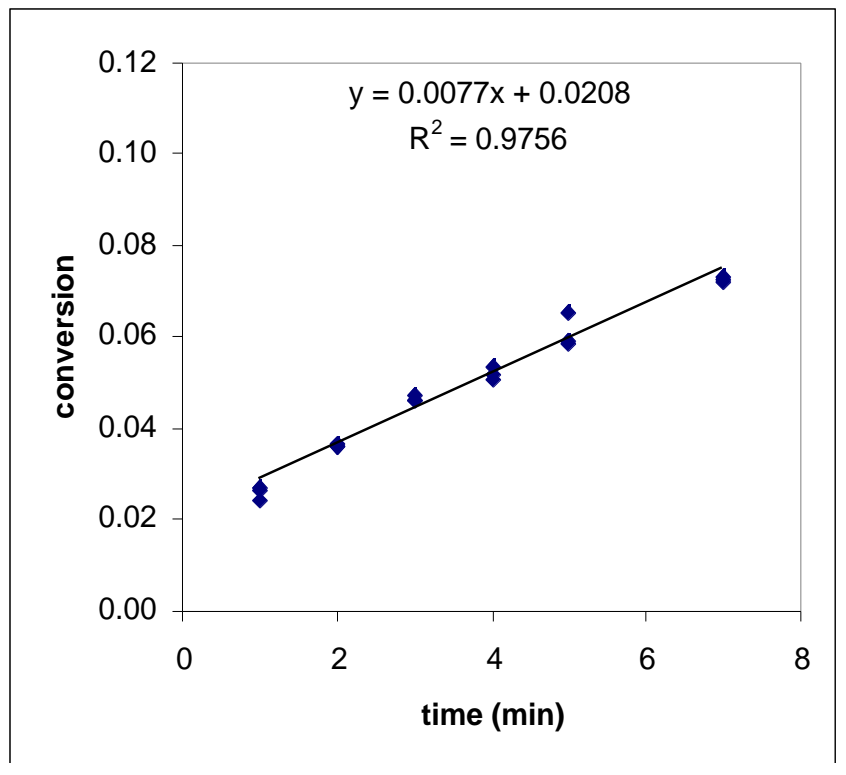




\begin{tabular}{|c|c|c|c|}
\hline time $(\min )$ & $\operatorname{area} \mathrm{A}$ & area 16 & conversion \\
\hline 1 & 7991 & 688 & 0.027 \\
\hline 1 & 8709 & 669 & 0.024 \\
\hline 1 & 8623 & 714 & 0.026 \\
\hline 2 & 7764 & 880 & 0.036 \\
\hline 2 & 7645 & 865 & 0.036 \\
\hline 2 & 7679 & 893 & 0.037 \\
\hline 3 & 8016 & 1202 & 0.048 \\
\hline 3 & 8110 & 1177 & 0.046 \\
\hline 3 & 7975 & 1163 & 0.046 \\
\hline 4 & 8349 & 1371 & 0.052 \\
\hline 4 & 8256 & 1401 & 0.054 \\
\hline 4 & 8373 & 1337 & 0.051 \\
\hline 5 & 4775 & 986 & 0.065 \\
\hline 5 & 4366 & 817 & 0.059 \\
\hline 5 & 4498 & 834 & 0.059 \\
\hline 7 & 7956 & 1824 & 0.073 \\
\hline 7 & 7834 & 1805 & 0.073 \\
\hline 7 & 8065 & 1836 & 0.072 \\
\hline 10 & 8165 & 2304 & 0.089 \\
\hline 10 & 9197 & 2534 & 0.087 \\
\hline 10 & 9247 & 2563 & 0.088 \\
\hline 15 & 6891 & 2397 & 0.110 \\
\hline 15 & 6837 & 2376 & 0.110 \\
\hline 15 & 6976 & 2418 & 0.110 \\
\hline 20 & 8054 & 3279 & 0.129 \\
\hline 20 & 8237 & 3278 & 0.126 \\
\hline 20 & 8040 & 3210 & 0.126 \\
\hline 30 & 7646 & 4019 & 0.167 \\
\hline 30 & 7767 & 4015 & 0.164 \\
\hline 30 & 7723 & 4046 & 0.166 \\
\hline 40 & 7371 & 4764 & 0.205 \\
\hline 40 & 7399 & 4749 & 0.203 \\
\hline 40 & 7353 & 4734 & 0.204 \\
\hline 50 & 6940 & 5386 & 0.246 \\
\hline 50 & 7090 & 5478 & 0.245 \\
\hline 50 & 7134 & 5499 & 0.244 \\
\hline 60 & 4684 & 4176 & 0.282 \\
\hline 60 & 4729 & 4202 & 0.281 \\
\hline 60 & 4723 & 4128 & 0.277 \\
\hline 75 & 6920 & 7516 & 0.344 \\
\hline 75 & 6962 & 7561 & 0.344 \\
\hline 75 & 6934 & 7604 & 0.347 \\
\hline 90 & 3880 & 4607 & 0.376 \\
\hline 90 & 3825 & 4617 & 0.382 \\
\hline 90 & 3863 & 4533 & 0.372 \\
\hline 120 & 1679 & 2587 & 0.488 \\
\hline 120 & 1684 & 2547 & 0.479 \\
\hline 120 & 1689 & 2635 & 0.494 \\
\hline
\end{tabular}


Order in $(E)-2,(1.0$ equiv $)$.

(E)-2

2-Iodothiophene

Tetra-n-butylammonium fluoride

Bis(dibenzylideneacetone)palladium $6.25 \mathrm{mM}$ (0.05 equiv)
$125 \mathrm{mM}$ (1.0 equiv)

$250 \mathrm{mM}$ (2.0 equiv)
$125 \mathrm{mM}$ (1.0 equiv)

Following the General Procedure, silanol (E)-2 (57.1 mg, $0.25 \mathrm{mmol})$ and biphenyl (10.4 $\mathrm{mg}$ ) were dissolved in TBAF solution $(500 \mu \mathrm{L}, 1.0 \mathrm{M}$ in THF, $0.5 \mathrm{mmol})$ in a flame dried 2-neck round-bottomed flask, equipped with a stir bar and a septum under argon. Dry THF $(0.7 \mathrm{~mL})$ was added and the clear, slightly-yellow solution was immersed into a water-bath at $23{ }^{\circ} \mathrm{C} \pm 1$ ${ }^{\circ} \mathrm{C}$ for $30 \mathrm{~min}$. The 2-iodothiophene $(28 \mu \mathrm{L}, 0.25 \mathrm{mmol})$ was then added and the reaction was stirred for $1 \mathrm{~min}$. A suspension of $\operatorname{Pd}(\mathrm{dba})_{2}(7.2 \mathrm{mg}, 0.0125 \mathrm{mmol})$ in dry THF $(1.0 \mathrm{~mL})$ was added. The initially purple, turbid reaction mixture turns into a clear solution within 30 seconds. Aliquots ( $200 \mathrm{uL}$ were withdrawn after 1, 2, 3, 4, 5, 7, 10, 15, 20, 30, 40, 50, 60, 75, 90, and $120 \mathrm{~min}$. To quench, these samples were added to $100 \mathrm{uL}$ of a $20 \%$ aqueous solution of 2dimethylaminoethanethiol hydrochloride. The clear colorless to slightly-yellow solution was then filtered through a plug of silica gel, and the silica gel was washed with diethyl ether to give a total sample volume of $\sim 2 \mathrm{~mL}$. This sample was injected into the GC three times.

initial rate $=0.0066$

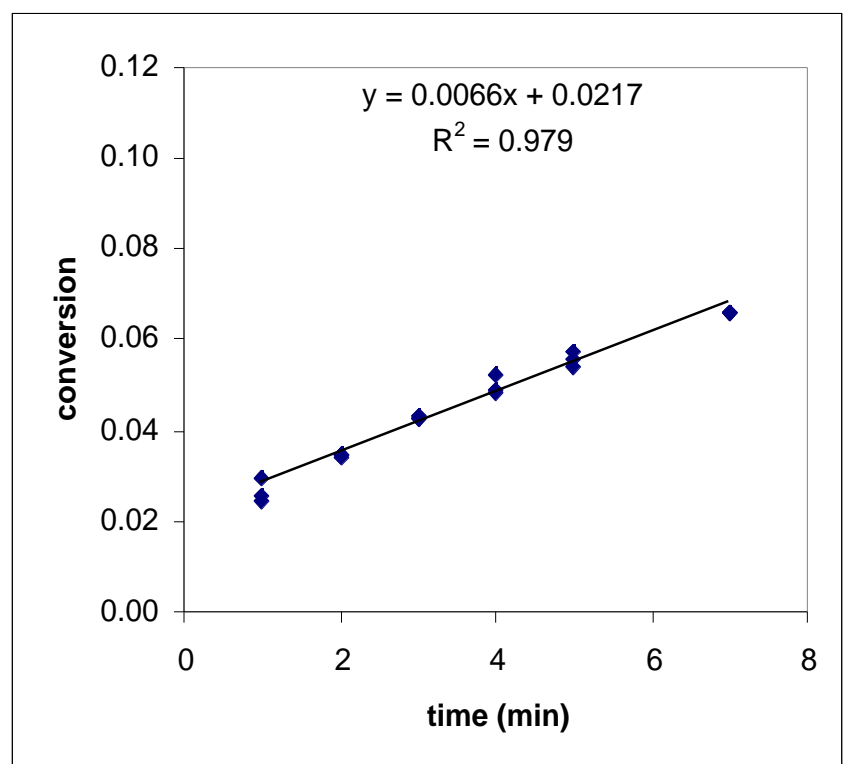




\begin{tabular}{|c|c|c|c|}
\hline time $(\min )$ & area $\mathrm{A}$ & area 16 & conversion \\
\hline 1 & 12970 & 978 & 0.030 \\
\hline 1 & 13117 & 849 & 0.025 \\
\hline 1 & 13010 & 812 & 0.024 \\
\hline 2 & 12394 & 1092 & 0.035 \\
\hline 2 & 12254 & 1092 & 0.035 \\
\hline 2 & 12180 & 1066 & 0.034 \\
\hline 3 & 10642 & 1161 & 0.043 \\
\hline 3 & 10843 & 1192 & 0.043 \\
\hline 3 & 10953 & 1190 & 0.043 \\
\hline 4 & 10980 & 1369 & 0.049 \\
\hline 4 & 10888 & 1447 & 0.052 \\
\hline 4 & 10813 & 1335 & 0.048 \\
\hline 5 & 12295 & 1753 & 0.056 \\
\hline 5 & 12340 & 1799 & 0.057 \\
\hline 5 & 12247 & 1681 & 0.054 \\
\hline 7 & 11209 & 1892 & 0.066 \\
\hline 7 & 11265 & 1902 & 0.066 \\
\hline 7 & 11190 & 1881 & 0.066 \\
\hline 10 & 11236 & 2307 & 0.081 \\
\hline 10 & 11133 & 2281 & 0.080 \\
\hline 10 & 11025 & 2296 & 0.082 \\
\hline 15 & 10850 & 2899 & 0.105 \\
\hline 15 & 10922 & 2934 & 0.105 \\
\hline 15 & 10950 & 3010 & 0.108 \\
\hline 20 & 12804 & 3998 & 0.122 \\
\hline 20 & 13042 & 3980 & 0.120 \\
\hline 20 & 12809 & 4003 & 0.123 \\
\hline 30 & 13168 & 5116 & 0.152 \\
\hline 30 & 13054 & 5022 & 0.151 \\
\hline 30 & 12987 & 5018 & 0.152 \\
\hline 40 & 11444 & 5541 & 0.190 \\
\hline 40 & 11437 & 5568 & 0.191 \\
\hline 40 & 11559 & 5714 & 0.194 \\
\hline 50 & 13248 & 7455 & 0.221 \\
\hline 50 & 13281 & 7615 & 0.225 \\
\hline 50 & 13260 & 7457 & 0.221 \\
\hline 60 & 9330 & 6243 & 0.262 \\
\hline 60 & 9290 & 6256 & 0.264 \\
\hline 60 & 9197 & 6180 & 0.264 \\
\hline 75 & 12757 & 9876 & 0.304 \\
\hline 75 & 12658 & 9821 & 0.304 \\
\hline 75 & 12571 & 9972 & 0.311 \\
\hline 90 & 11328 & 10325 & 0.357 \\
\hline 90 & 11154 & 10398 & 0.366 \\
\hline 90 & 11257 & 10533 & 0.367 \\
\hline 120 & 15057 & 17698 & 0.461 \\
\hline 120 & 15100 & 17697 & 0.460 \\
\hline 120 & 15105 & 17763 & 0.461 \\
\hline
\end{tabular}


Order in (E)-2, (1.0 equiv).

(E)-2

2-Iodothiophene

Tetra-n-butylammonium fluoride

Bis(dibenzylideneacetone)palladium $6.25 \mathrm{mM}$ (0.05 equiv)
$125 \mathrm{mM}$ (1.0 equiv)

$125 \mathrm{mM}$ (1.0 equiv)

$250 \mathrm{mM}$ (2.0 equiv)

Following the General Procedure, silanol $(E)-2(57.1 \mathrm{mg}, 0.25 \mathrm{mmol})$ and biphenyl $(7.2$ $\mathrm{mg}$ ) were dissolved in TBAF solution $(500 \mu \mathrm{L}, 1.0 \mathrm{M}$ in THF, $0.5 \mathrm{mmol})$ in a flame dried 2-neck round-bottomed flask, equipped with a stir bar and a septum under argon. Dry THF $(0.7 \mathrm{~mL})$ was added and the clear, slightly-yellow solution was immersed into a water-bath at $23{ }^{\circ} \mathrm{C} \pm 1$ ${ }^{\circ} \mathrm{C}$ for $30 \mathrm{~min}$. The 2-iodothiophene $(28 \mu \mathrm{L}, 0.25 \mathrm{mmol})$ was then added and the reaction was stirred for $1 \mathrm{~min}$. A suspension of $\operatorname{Pd}(\mathrm{dba})_{2}(7.2 \mathrm{mg}, 0.0125 \mathrm{mmol})$ in dry THF $(1.0 \mathrm{~mL})$ was added. The initially purple, turbid reaction mixture turns into a clear solution within 30 seconds. Aliquots ( $200 \mathrm{uL}$ were withdrawn after 1, 2, 3, 4, 5, 7, 10, 15, 20, 30, 40, 50, 60, 75, 90, and 120 min. To quench, these samples were added to $100 \mathrm{uL}$ of a $20 \%$ aqueous solution of 2dimethylaminoethanethiol hydrochloride. The clear colorless to slightly-yellow solution was then filtered through a plug of silica gel, and the silica gel was washed with diethyl ether to give a total sample volume of $\sim 2 \mathrm{~mL}$. This sample was injected into the GC three times.

initial rate $=0.0079$

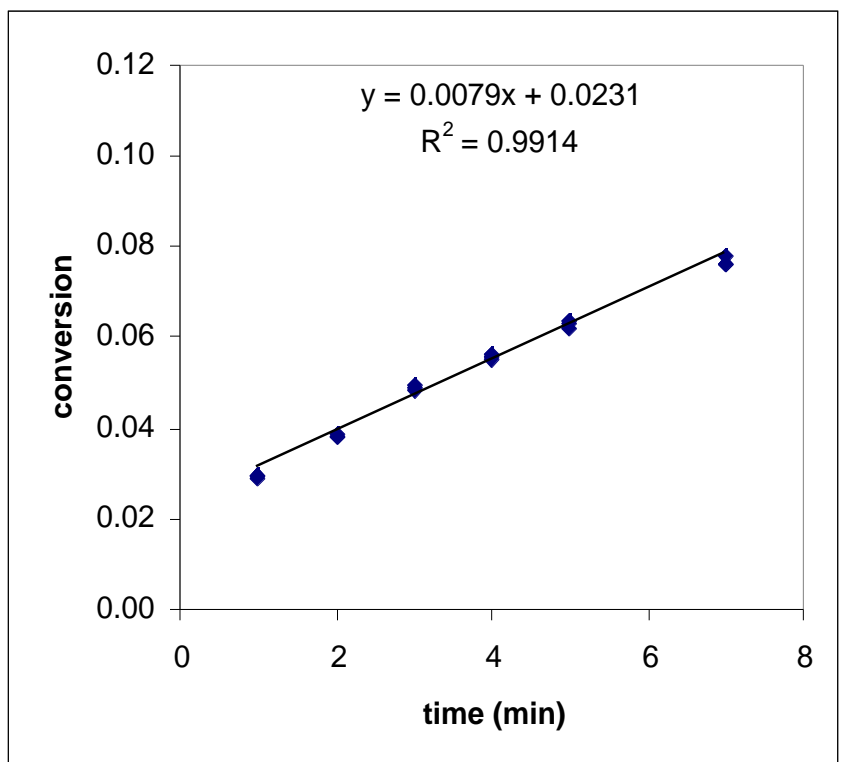




\begin{tabular}{|c|c|c|c|}
\hline time (min) & area $\mathrm{A}$ & area 16 & conversion \\
\hline 1 & 6411 & 703 & 0.030 \\
\hline 1 & 6507 & 708 & 0.030 \\
\hline 1 & 6483 & 689 & 0.029 \\
\hline 2 & 8164 & 1149 & 0.038 \\
\hline 2 & 8224 & 1177 & 0.039 \\
\hline 2 & 8187 & 1142 & 0.038 \\
\hline 3 & 6997 & 1243 & 0.048 \\
\hline 3 & 7042 & 1283 & 0.049 \\
\hline 3 & 6936 & 1245 & 0.049 \\
\hline 4 & 7881 & 1601 & 0.055 \\
\hline 4 & 7696 & 1572 & 0.055 \\
\hline 4 & 7719 & 1608 & 0.057 \\
\hline 5 & 7837 & 1820 & 0.063 \\
\hline 5 & 7713 & 1762 & 0.062 \\
\hline 5 & 7728 & 1808 & 0.064 \\
\hline 7 & 9741 & 2790 & 0.078 \\
\hline 7 & 9720 & 2730 & 0.076 \\
\hline 7 & 9666 & 2717 & 0.076 \\
\hline 10 & 6918 & 2440 & 0.096 \\
\hline 10 & 6876 & 2391 & 0.094 \\
\hline 10 & 6816 & 2395 & 0.095 \\
\hline 15 & 6983 & 3200 & 0.124 \\
\hline 15 & 6920 & 3188 & 0.125 \\
\hline 15 & 6995 & 3214 & 0.125 \\
\hline 20 & 7167 & 3968 & 0.150 \\
\hline 20 & 7077 & 3919 & 0.150 \\
\hline 20 & 7053 & 3925 & 0.151 \\
\hline 30 & 7615 & 5548 & 0.198 \\
\hline 30 & 7605 & 5484 & 0.196 \\
\hline 30 & 7580 & 5532 & 0.198 \\
\hline 40 & 7208 & 6652 & 0.251 \\
\hline 40 & 7153 & 6621 & 0.251 \\
\hline 40 & 7155 & 6526 & 0.248 \\
\hline 50 & 6847 & 7281 & 0.289 \\
\hline 50 & 6925 & 7251 & 0.284 \\
\hline 50 & 6929 & 7272 & 0.285 \\
\hline 60 & 7950 & 10148 & 0.347 \\
\hline 60 & 7953 & 10138 & 0.346 \\
\hline 60 & 8022 & 10026 & 0.339 \\
\hline 75 & 7064 & 10813 & 0.416 \\
\hline 75 & 7029 & 10755 & 0.415 \\
\hline 75 & 7015 & 10724 & 0.415 \\
\hline 90 & 8960 & 15748 & 0.477 \\
\hline 90 & 9142 & 16062 & 0.477 \\
\hline 90 & 9002 & 16053 & 0.484 \\
\hline 120 & 9329 & 21268 & 0.619 \\
\hline 120 & 9081 & 20507 & 0.613 \\
\hline 120 & 9053 & 20399 & 0.612 \\
\hline
\end{tabular}




\section{Order in $(E)-2,(1.0$ equiv $)$.}

(E)-2

2-Iodothiophene

Tetra-n-butylammonium fluoride

Bis(dibenzylideneacetone)palladium $6.25 \mathrm{mM}$ (0.05 equiv)
$125 \mathrm{mM}$ (1.0 equiv)

$125 \mathrm{mM}$ (1.0 equiv)

$250 \mathrm{mM}$ (2.0 equiv)

Following the General Procedure, silanol (E)-2 $(57.1 \mathrm{mg}, 0.25 \mathrm{mmol})$ and biphenyl $(9.2$ $\mathrm{mg}$ ) were dissolved in TBAF solution $(500 \mu \mathrm{L}, 1.0 \mathrm{M}$ in THF, $0.5 \mathrm{mmol})$ in a flame dried 2-neck round-bottomed flask, equipped with a stir bar and a septum under argon. Dry THF $(0.7 \mathrm{~mL})$ was added and the clear, slightly-yellow solution was immersed into a water-bath at $23{ }^{\circ} \mathrm{C} \pm 1$ ${ }^{\circ} \mathrm{C}$ for $30 \mathrm{~min}$. The 2-iodothiophene $(28 \mu \mathrm{L}, 0.25 \mathrm{mmol})$ was then added and the reaction was stirred for $1 \mathrm{~min}$. A suspension of $\operatorname{Pd}(\mathrm{dba})_{2}(7.2 \mathrm{mg}, 0.0125 \mathrm{mmol})$ in dry THF $(1.0 \mathrm{~mL})$ was added. The initially purple, turbid reaction mixture turns into a clear solution within 30 seconds. Aliquots ( $200 \mathrm{uL}$ were withdrawn after 1, 2, 3, 4, 5, 7, 10, 15, 20, 30, 40, 50, 60, 75, 90, and $120 \mathrm{~min}$. To quench, these samples were added to $100 \mathrm{uL}$ of a $20 \%$ aqueous solution of 2dimethylaminoethanethiol hydrochloride. The clear colorless to slightly-yellow solution was then filtered through a plug of silica gel, and the silica gel was washed with diethyl ether to give a total sample volume of $\sim 2 \mathrm{~mL}$. This sample was injected into the GC three times.

initial rate $=0.0082$

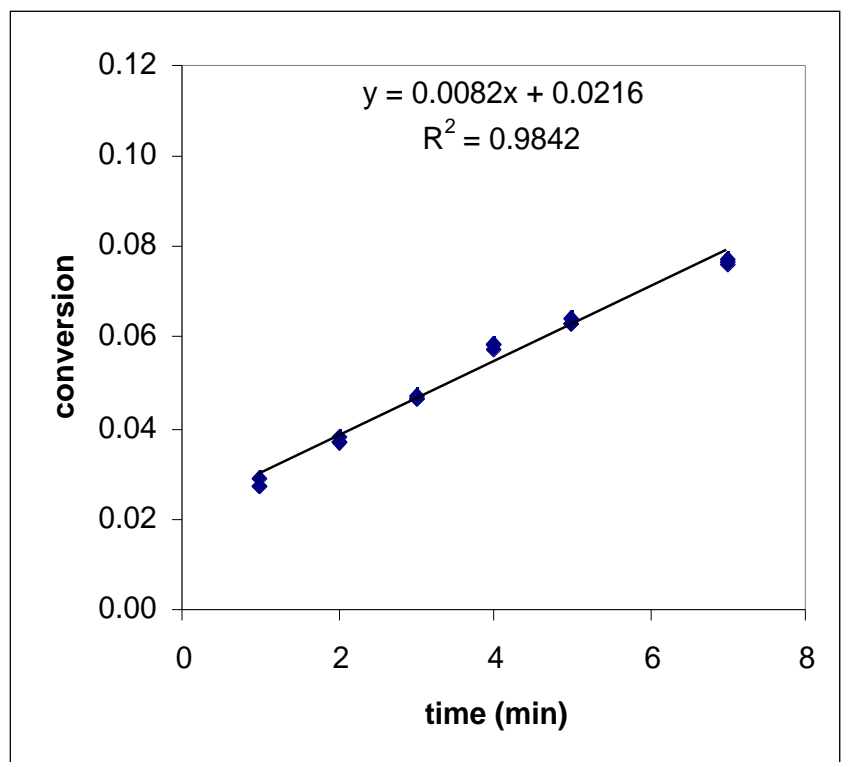




\begin{tabular}{|c|c|c|c|}
\hline time $(\min )$ & area $\mathrm{A}$ & area 16 & conversion \\
\hline 1 & 10109 & 837 & 0.029 \\
\hline 1 & 9940 & 783 & 0.027 \\
\hline 1 & 9966 & 789 & 0.027 \\
\hline 2 & 12187 & 1338 & 0.038 \\
\hline 2 & 12035 & 1290 & 0.037 \\
\hline 2 & 12077 & 1292 & 0.037 \\
\hline 3 & 10984 & 1497 & 0.047 \\
\hline 3 & 10971 & 1478 & 0.047 \\
\hline 3 & 11055 & 1487 & 0.047 \\
\hline 4 & 10282 & 1694 & 0.057 \\
\hline 4 & 10492 & 1769 & 0.058 \\
\hline 4 & 10460 & 1764 & 0.059 \\
\hline 5 & 11434 & 2073 & 0.063 \\
\hline 5 & 11480 & 2127 & 0.064 \\
\hline 5 & 11428 & 2070 & 0.063 \\
\hline 7 & 10893 & 2395 & 0.076 \\
\hline 7 & 10872 & 2423 & 0.077 \\
\hline 7 & 10880 & 2404 & 0.077 \\
\hline 10 & 11004 & 3030 & 0.096 \\
\hline 10 & 10744 & 2955 & 0.095 \\
\hline 10 & 10706 & 2969 & 0.096 \\
\hline 15 & 11325 & 3958 & 0.121 \\
\hline 15 & 11373 & 3932 & 0.120 \\
\hline 15 & 11210 & 3828 & 0.118 \\
\hline 20 & 9331 & 3852 & 0.143 \\
\hline 20 & 9314 & 3850 & 0.143 \\
\hline 20 & 10111 & 4337 & 0.149 \\
\hline 30 & 13634 & 7398 & 0.188 \\
\hline 30 & 13746 & 7489 & 0.189 \\
\hline 30 & 13263 & 7091 & 0.185 \\
\hline 40 & 10133 & 6850 & 0.235 \\
\hline 40 & 10461 & 7041 & 0.234 \\
\hline 40 & 10201 & 6925 & 0.236 \\
\hline 50 & 10520 & 8313 & 0.274 \\
\hline 50 & 10381 & 8241 & 0.275 \\
\hline 50 & 10664 & 8345 & 0.272 \\
\hline 60 & 5484 & 4824 & 0.305 \\
\hline 60 & 5677 & 4993 & 0.305 \\
\hline 60 & 5667 & 5047 & 0.309 \\
\hline 75 & 12162 & 13193 & 0.376 \\
\hline 75 & 12026 & 13230 & 0.382 \\
\hline 75 & 12383 & 13413 & 0.376 \\
\hline 90 & 11091 & 14106 & 0.441 \\
\hline 90 & 11166 & 14214 & 0.442 \\
\hline 90 & 11259 & 14109 & 0.435 \\
\hline 120 & 12311 & 20163 & 0.568 \\
\hline 120 & 12591 & 20648 & 0.569 \\
\hline 120 & 12379 & 20342 & 0.570 \\
\hline
\end{tabular}




\section{Order in $(E)-2,(1.5$ equiv).}

(E)-2

2-Iodothiophene

Tetra-n-butylammonium fluoride

Bis(dibenzylideneacetone)palladium $6.25 \mathrm{mM}$ (0.05 equiv)
$188 \mathrm{mM}$ (1.5 equiv)

$125 \mathrm{mM}$ (1.0 equiv)

$250 \mathrm{mM}$ ( 2.0 equiv)

Following the General Procedure, silanol (E)-2 $(85.7 \mathrm{mg}, 0.375 \mathrm{mmol})$ and biphenyl (23.5 mg) were dissolved in TBAF solution $(500 \mu \mathrm{L}, 1.0 \mathrm{M}$ in THF, $0.5 \mathrm{mmol})$ in a flame dried 2-neck round-bottomed flask, equipped with a stir bar and a septum under argon. Dry THF (0.7 $\mathrm{mL}$ ) was added and the clear, slightly-yellow solution was immersed into a water-bath at $23{ }^{\circ} \mathrm{C} \pm$ $1{ }^{\circ} \mathrm{C}$ for $30 \mathrm{~min}$. The 2-iodothiophene $(28 \mu \mathrm{L}, 0.25 \mathrm{mmol})$ was then added and the reaction was stirred for $1 \mathrm{~min}$. A suspension of $\operatorname{Pd}(\mathrm{dba})_{2}(7.2 \mathrm{mg}, 0.0125 \mathrm{mmol})$ in dry THF $(1.0 \mathrm{~mL})$ was added. The initially purple, turbid reaction mixture turns into a clear solution within 30 seconds. Aliquots $(\sim 200 \mathrm{uL}$ were withdrawn after 1, 2, 3, 4, 5, 7, 10, 15, 20, 30, 40, 50, and $60 \mathrm{~min}$. To quench, these samples were added to $100 \mathrm{uL}$ of a $20 \%$ aqueous solution of 2dimethylaminoethanethiol hydrochloride. The clear colorless to slightly-yellow solution was then filtered through a plug of silica gel, and the silica gel was washed with diethyl ether to give a total sample volume of $\sim 2 \mathrm{~mL}$. This sample was injected into the GC three times.

initial rate $=0.0261$

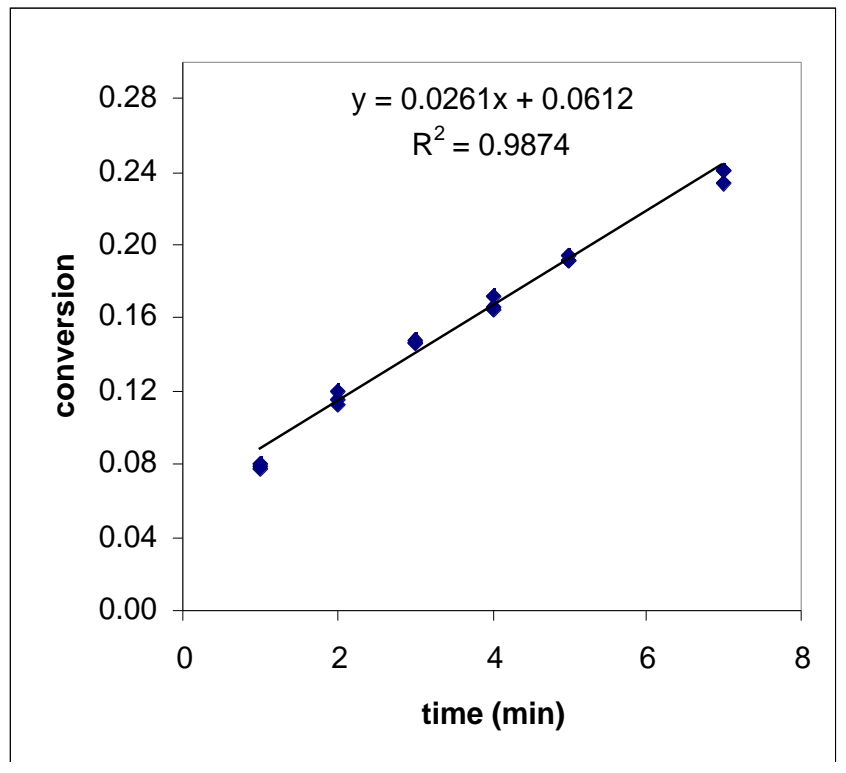




\begin{tabular}{|c|c|c|c|}
\hline time (min) & area $\mathrm{A}$ & area 16 & conversion \\
\hline 1 & 8464 & 1124 & 0.078 \\
\hline 1 & 8503 & 1113 & 0.077 \\
\hline 1 & 8565 & 1161 & 0.080 \\
\hline 2 & 6686 & 1270 & 0.112 \\
\hline 2 & 6492 & 1267 & 0.115 \\
\hline 2 & 6679 & 1351 & 0.120 \\
\hline 3 & 6046 & 1492 & 0.146 \\
\hline 3 & 6081 & 1529 & 0.149 \\
\hline 3 & 6079 & 1513 & 0.147 \\
\hline 4 & 6412 & 1788 & 0.165 \\
\hline 4 & 6361 & 1794 & 0.167 \\
\hline 4 & 6328 & 1838 & 0.172 \\
\hline 5 & 7546 & 2442 & 0.191 \\
\hline 5 & 7520 & 2480 & 0.195 \\
\hline 5 & 7668 & 2478 & 0.191 \\
\hline 7 & 7757 & 3077 & 0.234 \\
\hline 7 & 7806 & 3187 & 0.241 \\
\hline 7 & 7729 & 3152 & 0.241 \\
\hline 10 & 8847 & 4438 & 0.296 \\
\hline 10 & 8929 & 4499 & 0.298 \\
\hline 10 & 9135 & 4395 & 0.284 \\
\hline 15 & 6937 & 4679 & 0.399 \\
\hline 15 & 7012 & 4769 & 0.402 \\
\hline 15 & 7053 & 4723 & 0.396 \\
\hline 20 & 7910 & 6508 & 0.486 \\
\hline 20 & 7415 & 6532 & 0.520 \\
\hline 20 & 7855 & 6622 & 0.498 \\
\hline 30 & 9210 & 10540 & 0.676 \\
\hline 30 & 9131 & 10363 & 0.671 \\
\hline 30 & 9155 & 10543 & 0.680 \\
\hline 40 & 6716 & 9927 & 0.873 \\
\hline 40 & 6649 & 9849 & 0.875 \\
\hline 40 & 6678 & 9722 & 0.860 \\
\hline 50 & 4348 & 7644 & 1.039 \\
\hline 50 & 4392 & 7631 & 1.027 \\
\hline 50 & 4326 & 7573 & 1.034 \\
\hline
\end{tabular}




\section{Order in $(E)-2$, (1.5 equiv).}

(E)-2

2-Iodothiophene

Tetra-n-butylammonium fluoride

Bis(dibenzylideneacetone)palladium $6.25 \mathrm{mM}$ (0.05 equiv)
$188 \mathrm{mM}(1.5$ equiv)

$125 \mathrm{mM}$ (1.0 equiv)

$250 \mathrm{mM}$ (2.0 equiv)

Following the General Procedure, silanol (E)-2 $(85.7 \mathrm{mg}, 0.375 \mathrm{mmol})$ and biphenyl (22.8 mg) were dissolved in TBAF solution $(500 \mu \mathrm{L}, 1.0 \mathrm{M}$ in THF, $0.5 \mathrm{mmol})$ in a flame dried 2-neck round-bottomed flask, equipped with a stir bar and a septum under argon. Dry THF (0.7 $\mathrm{mL}$ ) was added and the clear, slightly-yellow solution was immersed into a water-bath at $23{ }^{\circ} \mathrm{C} \pm$ $1{ }^{\circ} \mathrm{C}$ for $30 \mathrm{~min}$. The 2-iodothiophene $(28 \mu \mathrm{L}, 0.25 \mathrm{mmol})$ was then added and the reaction was stirred for $1 \mathrm{~min}$. A suspension of $\operatorname{Pd}(\mathrm{dba})_{2}(7.2 \mathrm{mg}, 0.0125 \mathrm{mmol})$ in dry THF $(1.0 \mathrm{~mL})$ was added. The initially purple, turbid reaction mixture turns into a clear solution within 30 seconds. Aliquots $(\sim 200 \mathrm{uL}$ were withdrawn after 1, 2, 3, 4, 5, 7, 10, 15, 20, 30, 40, 50, and $60 \mathrm{~min}$. To quench, these samples were added to $100 \mathrm{uL}$ of a $20 \%$ aqueous solution of 2dimethylaminoethanethiol hydrochloride. The clear colorless to slightly-yellow solution was then filtered through a plug of silica gel, and the silica gel was washed with diethyl ether to give a total sample volume of $\sim 2 \mathrm{~mL}$. This sample was injected into the $\mathrm{GC}$ three times.

initial rate $=0.0291$

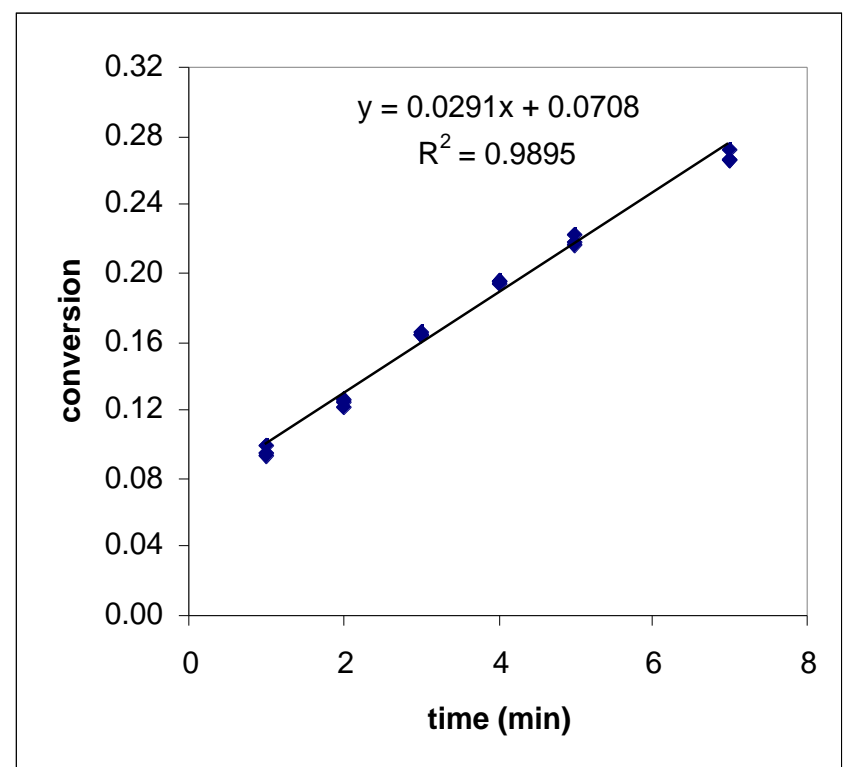




\begin{tabular}{|c|c|c|c|}
\hline time $(\min )$ & area $\mathrm{A}$ & area 16 & conversion \\
\hline 1 & 8338 & 1348 & 0.093 \\
\hline 1 & 8496 & 1399 & 0.094 \\
\hline 1 & 8143 & 1404 & 0.099 \\
\hline 2 & 4637 & 1022 & 0.126 \\
\hline 2 & 4641 & 1012 & 0.125 \\
\hline 2 & 4633 & 983 & 0.122 \\
\hline 3 & 7590 & 2181 & 0.165 \\
\hline 3 & 7526 & 2149 & 0.164 \\
\hline 3 & 7610 & 2168 & 0.163 \\
\hline 4 & 10286 & 3502 & 0.195 \\
\hline 4 & 10378 & 3511 & 0.194 \\
\hline 4 & 10256 & 3497 & 0.195 \\
\hline 5 & 8768 & 3315 & 0.217 \\
\hline 5 & 8531 & 3303 & 0.222 \\
\hline 5 & 8592 & 3260 & 0.217 \\
\hline 7 & 6587 & 3050 & 0.265 \\
\hline 7 & 6627 & 3076 & 0.266 \\
\hline 7 & 6356 & 3022 & 0.273 \\
\hline 10 & 7361 & 4324 & 0.337 \\
\hline 10 & 7464 & 4462 & 0.343 \\
\hline 10 & 7335 & 4416 & 0.345 \\
\hline 15 & 8564 & 6671 & 0.447 \\
\hline 15 & 8496 & 6550 & 0.442 \\
\hline 15 & 8164 & 6623 & 0.465 \\
\hline 20 & 8832 & 8374 & 0.544 \\
\hline 20 & 8913 & 8351 & 0.537 \\
\hline 20 & 8848 & 8364 & 0.542 \\
\hline 30 & 9497 & 12460 & 0.752 \\
\hline 30 & 9257 & 12343 & 0.764 \\
\hline 30 & 9180 & 12462 & 0.778 \\
\hline 40 & 9156 & 15826 & 0.991 \\
\hline 40 & 9141 & 15540 & 0.975 \\
\hline 40 & 9390 & 15771 & 0.963 \\
\hline
\end{tabular}




\section{Order in $(E)-2,(1.5$ equiv $)$.}

(E)-2

2-Iodothiophene

Tetra-n-butylammonium fluoride

Bis(dibenzylideneacetone)palladium $6.25 \mathrm{mM}$ (0.05 equiv)
$188 \mathrm{mM}(1.5$ equiv)

$125 \mathrm{mM}$ (1.0 equiv)

$250 \mathrm{mM}$ (2.0 equiv)

Following the General Procedure, silanol (E)-2 $(85.7 \mathrm{mg}, 0.375 \mathrm{mmol})$ and biphenyl (21.7 mg) were dissolved in TBAF solution $(500 \mu \mathrm{L}, 1.0 \mathrm{M}$ in THF, $0.5 \mathrm{mmol})$ in a flame dried 2-neck round-bottomed flask, equipped with a stir bar and a septum under argon. Dry THF (0.7 $\mathrm{mL}$ ) was added and the clear, slightly-yellow solution was immersed into a water-bath at $23{ }^{\circ} \mathrm{C} \pm$ $1{ }^{\circ} \mathrm{C}$ for $30 \mathrm{~min}$. The 2-iodothiophene $(28 \mu \mathrm{L}, 0.25 \mathrm{mmol})$ was then added and the reaction was stirred for $1 \mathrm{~min}$. A suspension of $\operatorname{Pd}(\mathrm{dba})_{2}(7.2 \mathrm{mg}, 0.0125 \mathrm{mmol})$ in dry THF $(1.0 \mathrm{~mL})$ was added. The initially purple, turbid reaction mixture turns into a clear solution within 30 seconds. Aliquots $(\sim 200 \mathrm{uL}$ were withdrawn after 1, 2, 3, 4, 5, 7, 10, 15, 20, 30, 40, 50, and $60 \mathrm{~min}$. To quench, these samples were added to $100 \mathrm{uL}$ of a $20 \%$ aqueous solution of 2dimethylaminoethanethiol hydrochloride. The clear colorless to slightly-yellow solution was then filtered through a plug of silica gel, and the silica gel was washed with diethyl ether to give a total sample volume of $\sim 2 \mathrm{~mL}$. This sample was injected into the $\mathrm{GC}$ three times.

initial rate $=0.0276$

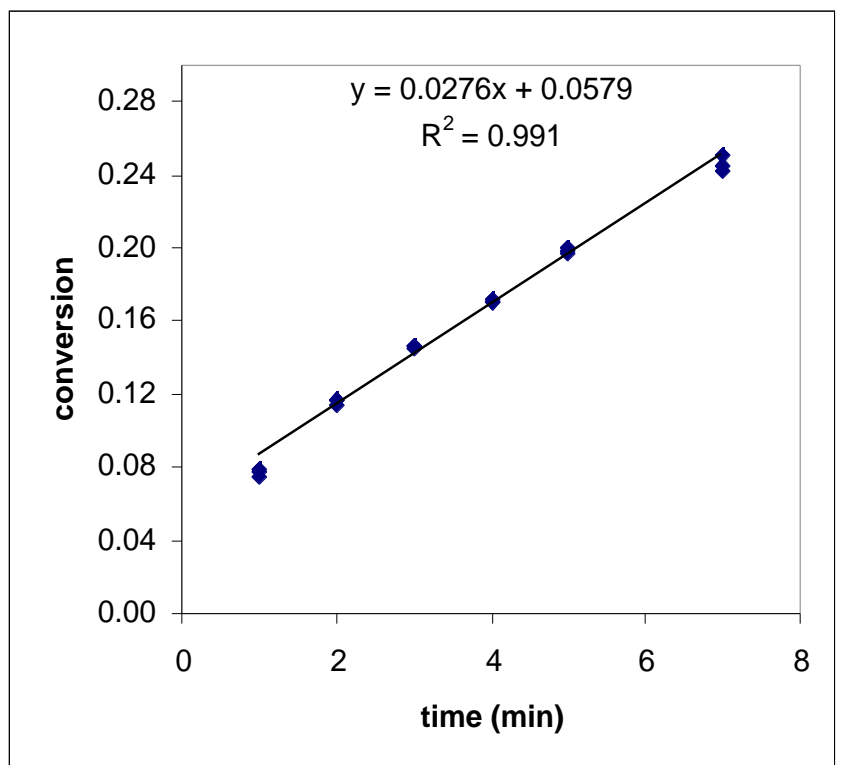




\begin{tabular}{|c|c|c|c|}
\hline time (min) & area $\mathrm{A}$ & area $\mathbf{1 6}$ & conversion \\
\hline 1 & 9393 & 1365 & 0.079 \\
\hline 1 & 9515 & 1311 & 0.075 \\
\hline 1 & 9337 & 1328 & 0.078 \\
\hline 2 & 8634 & 1797 & 0.114 \\
\hline 2 & 8642 & 1858 & 0.117 \\
\hline 2 & 8640 & 1858 & 0.117 \\
\hline 3 & 9564 & 2547 & 0.145 \\
\hline 3 & 9468 & 2550 & 0.147 \\
\hline 3 & 9617 & 2580 & 0.146 \\
\hline 4 & 8014 & 2505 & 0.171 \\
\hline 4 & 7931 & 2492 & 0.171 \\
\hline 4 & 8012 & 2509 & 0.171 \\
\hline 5 & 10036 & 3645 & 0.198 \\
\hline 5 & 10014 & 3629 & 0.198 \\
\hline 5 & 10076 & 3695 & 0.200 \\
\hline 7 & 8733 & 4008 & 0.250 \\
\hline 7 & 8918 & 4006 & 0.245 \\
\hline 7 & 8926 & 3972 & 0.243 \\
\hline 10 & 8532 & 4774 & 0.305 \\
\hline 10 & 8635 & 4847 & 0.306 \\
\hline 10 & 8706 & 4841 & 0.303 \\
\hline 15 & 10580 & 7829 & 0.404 \\
\hline 15 & 10388 & 7729 & 0.406 \\
\hline 15 & 10355 & 7781 & 0.410 \\
\hline 20 & 8684 & 7949 & 0.499 \\
\hline 20 & 8735 & 7785 & 0.486 \\
\hline 20 & 8644 & 7813 & 0.493 \\
\hline 30 & 9257 & 11844 & 0.698 \\
\hline 30 & 9431 & 12015 & 0.695 \\
\hline 30 & 9541 & 12207 & 0.698 \\
\hline 40 & 8924 & 14100 & 0.862 \\
\hline 40 & 8921 & 14071 & 0.861 \\
\hline 40 & 8939 & 13886 & 0.848 \\
\hline 50 & 9843 & 18485 & 1.025 \\
\hline 50 & 9390 & 17781 & 1.033 \\
\hline 50 & 9736 & 18522 & 1.038 \\
\hline
\end{tabular}


Order in (E)-2, (2.0 equiv).

(E)-2

2-Iodothiophene

Tetra-n-butylammonium fluoride

Bis(dibenzylideneacetone)palladium $6.25 \mathrm{mM}$ (0.05 equiv)
$250 \mathrm{mM}$ (2.0 equiv)

$125 \mathrm{mM}$ (1.0 equiv)

$250 \mathrm{mM}$ (2.0 equiv)

Following the General Procedure, silanol (E)-2 (114.2 mg, $0.5 \mathrm{mmol})$ and biphenyl (23.2 $\mathrm{mg}$ ) were dissolved in TBAF solution $((500 \mu \mathrm{L}, 1.0 \mathrm{M}$ in THF, $0.5 \mathrm{mmol})$ in a flame dried 2neck round-bottomed flask, equipped with a stir bar and a septum under argon. Dry THF (0.7 $\mathrm{mL}$ ) was added and the clear, slightly-yellow solution was immersed into a water-bath at $23{ }^{\circ} \mathrm{C} \pm$ $1{ }^{\circ} \mathrm{C}$ for $30 \mathrm{~min}$. The 2-iodothiophene $(28 \mu \mathrm{L}, 0.25 \mathrm{mmol})$ was then added and the reaction was stirred for $1 \mathrm{~min}$. A suspension of $\operatorname{Pd}(\mathrm{dba})_{2}(7.2 \mathrm{mg}, 0.0125 \mathrm{mmol})$ in dry THF $(1.0 \mathrm{~mL})$ was added. The initially purple, turbid reaction mixture turns into a clear solution within 30 seconds. Aliquots $(\sim 200 \mu \mathrm{L}$ )were withdrawn after 1, 2, 3, 4, 5, 7, 10, 15, 20, 30, 40, 50, 60, 75, 90, and $120 \mathrm{~min}$. To quench, these samples were added to $100 \mu \mathrm{L}$ of a $20 \%$ aqueous solution of 2dimethylaminoethanethiol hydrochloride. The clear colorless to slightly-yellow solution was then filtered through a plug of silica gel, and the silica gel was washed with diethyl ether to give a total sample volume of $\sim 2 \mathrm{~mL}$. This sample was injected into the GC three times.

initial rate $=0.0354$

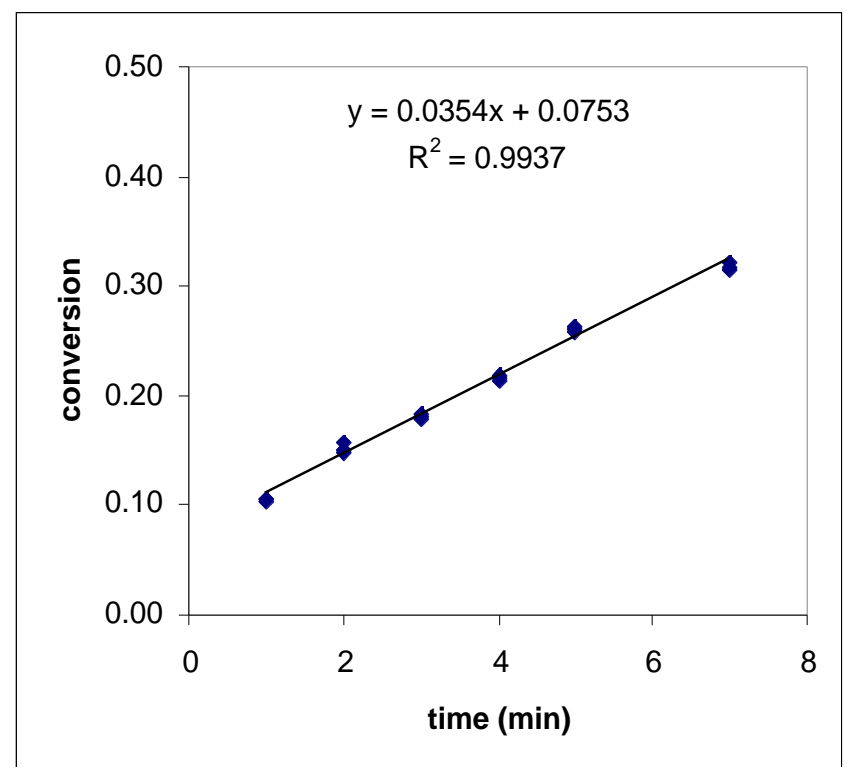




\begin{tabular}{|c|c|c|c|}
\hline time $(\min )$ & area $\mathrm{A}$ & area 16 & conversion \\
\hline 1 & 7041 & 852 & 0.106 \\
\hline 1 & 6674 & 804 & 0.105 \\
\hline 1 & 6930 & 816 & 0.103 \\
\hline 2 & 5582 & 997 & 0.156 \\
\hline 2 & 6349 & 1097 & 0.151 \\
\hline 2 & 7626 & 1289 & 0.148 \\
\hline 3 & 29546 & 6147 & 0.182 \\
\hline 3 & 50964 & 10367 & 0.178 \\
\hline 3 & 46323 & 9520 & 0.180 \\
\hline 4 & 63852 & 15639 & 0.214 \\
\hline 4 & 35537 & 8871 & 0.218 \\
\hline 4 & 31440 & 7782 & 0.217 \\
\hline 5 & 9003 & 2675 & 0.260 \\
\hline 5 & 8628 & 2587 & 0.262 \\
\hline 5 & 9266 & 2724 & 0.257 \\
\hline 7 & 11868 & 4358 & 0.321 \\
\hline 7 & 10832 & 3908 & 0.316 \\
\hline 7 & 14560 & 5269 & 0.317 \\
\hline 10 & 14426 & 6580 & 0.399 \\
\hline 10 & 11942 & 5422 & 0.397 \\
\hline 10 & 13406 & 6108 & 0.399 \\
\hline 15 & 14213 & 8619 & 0.531 \\
\hline 15 & 13250 & 8117 & 0.536 \\
\hline 15 & 13793 & 8387 & 0.532 \\
\hline 20 & 11003 & 8177 & 0.650 \\
\hline 20 & 10768 & 8042 & 0.653 \\
\hline 20 & 11086 & 8257 & 0.652 \\
\hline 30 & 16423 & 16797 & 0.895 \\
\hline 30 & 15569 & 15909 & 0.894 \\
\hline 30 & 23736 & 24160 & 0.891 \\
\hline
\end{tabular}


Order in (E)-2, (2.0 equiv).

(E)-2

2-Iodothiophene

Tetra-n-butylammonium fluoride

Bis(dibenzylideneacetone)palladium $6.25 \mathrm{mM}$ (0.05 equiv)
$250 \mathrm{mM}$ (2.0 equiv)

$125 \mathrm{mM}$ (1.0 equiv)

$250 \mathrm{mM}$ (2.0 equiv)

Following the General Procedure, silanol (E)-2 (114.2 mg, $0.5 \mathrm{mmol})$ and biphenyl (25.3 $\mathrm{mg}$ ) were dissolved in TBAF solution $((500 \mu \mathrm{L}, 1.0 \mathrm{M}$ in THF, $0.5 \mathrm{mmol})$ in a flame dried 2neck round-bottomed flask, equipped with a stir bar and a septum under argon. Dry THF (0.7 $\mathrm{mL}$ ) was added and the clear, slightly-yellow solution was immersed into a water-bath at $23{ }^{\circ} \mathrm{C} \pm$ $1{ }^{\circ} \mathrm{C}$ for $30 \mathrm{~min}$. The 2-iodothiophene $(28 \mu \mathrm{L}, 0.25 \mathrm{mmol})$ was then added and the reaction was stirred for $1 \mathrm{~min}$. A suspension of $\operatorname{Pd}(\mathrm{dba})_{2}(7.2 \mathrm{mg}, 0.0125 \mathrm{mmol})$ in dry THF $(1.0 \mathrm{~mL})$ was added. The initially purple, turbid reaction mixture turns into a clear solution within 30 seconds. Aliquots $(\sim 200 \mu \mathrm{L}$ )were withdrawn after 1, 2, 3, 4, 5, 7, 10, 15, 20, 30, 40, 50, 60, 75, 90, and $120 \mathrm{~min}$. To quench, these samples were added to $100 \mu \mathrm{L}$ of a $20 \%$ aqueous solution of 2dimethylaminoethanethiol hydrochloride. The clear colorless to slightly-yellow solution was then filtered through a plug of silica gel, and the silica gel was washed with diethyl ether to give a total sample volume of $\sim 2 \mathrm{~mL}$. This sample was injected into the GC three times.

initial rate $=0.0378$

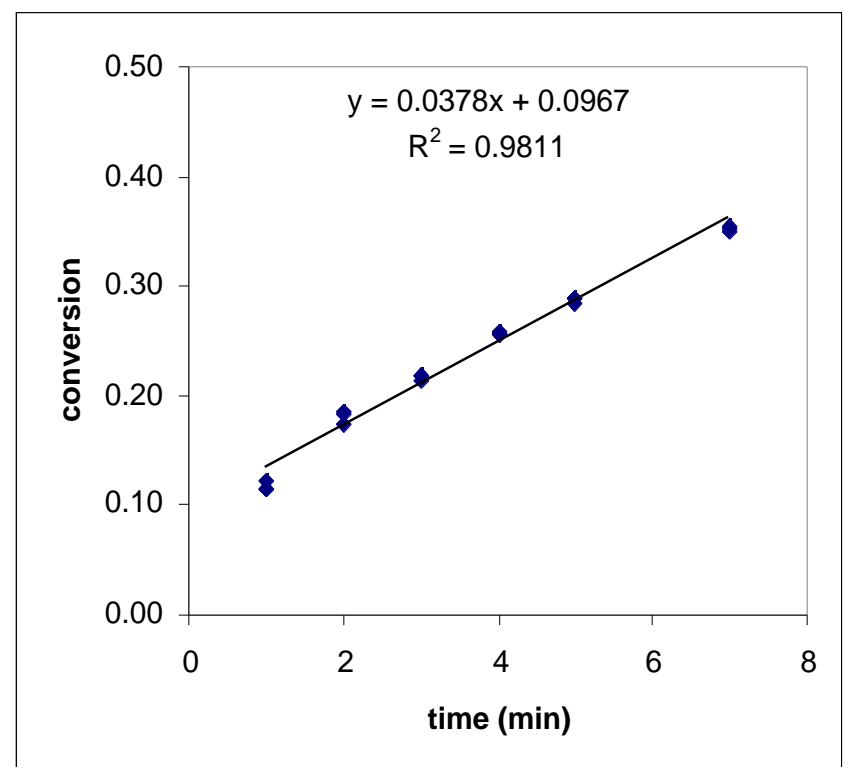




\begin{tabular}{|c|c|c|c|}
\hline time (min) & area $\mathrm{A}$ & area 16 & conversion \\
\hline 1 & 30783 & 3945 & 0.122 \\
\hline 1 & 56311 & 6802 & 0.115 \\
\hline 1 & 47032 & 5676 & 0.115 \\
\hline 2 & 12209 & 2357 & 0.184 \\
\hline 2 & 19695 & 3813 & 0.185 \\
\hline 2 & 11378 & 2062 & 0.173 \\
\hline 3 & 9069 & 2083 & 0.219 \\
\hline 3 & 11171 & 2508 & 0.214 \\
\hline 3 & 7969 & 1832 & 0.219 \\
\hline 4 & 7575 & 2027 & 0.255 \\
\hline 4 & 8894 & 2394 & 0.257 \\
\hline 4 & 8774 & 2384 & 0.259 \\
\hline 5 & 11802 & 3579 & 0.289 \\
\hline 5 & 13251 & 3997 & 0.288 \\
\hline 5 & 19138 & 5712 & 0.285 \\
\hline 7 & 16212 & 5958 & 0.351 \\
\hline 7 & 11825 & 4351 & 0.351 \\
\hline 7 & 11544 & 4285 & 0.354 \\
\hline 10 & 13682 & 6440 & 0.449 \\
\hline 10 & 13000 & 6500 & 0.477 \\
\hline 10 & 13132 & 6455 & 0.469 \\
\hline 15 & 12621 & 7852 & 0.594 \\
\hline 15 & 13995 & 8591 & 0.586 \\
\hline 15 & 36197 & 22059 & 0.581 \\
\hline 20 & 32197 & 24266 & 0.719 \\
\hline 20 & 40273 & 30138 & 0.714 \\
\hline 20 & 38875 & 29226 & 0.717 \\
\hline 30 & 77173 & 77748 & 0.961 \\
\hline 30 & 16404 & 16994 & 0.988 \\
\hline 30 & 16825 & 17316 & 0.982 \\
\hline
\end{tabular}


Order in (E)-2, (2.0 equiv).

(E)-2

2-Iodothiophene

Tetra-n-butylammonium fluoride

Bis(dibenzylideneacetone)palladium $6.25 \mathrm{mM}$ (0.05 equiv)
$250 \mathrm{mM}$ (2.0 equiv)

$125 \mathrm{mM}$ (1.0 equiv)

$250 \mathrm{mM}$ (2.0 equiv)

Following the General Procedure, silanol (E)-2 (114.2 mg, $0.5 \mathrm{mmol})$ and biphenyl (21.2 $\mathrm{mg}$ ) were dissolved in TBAF solution $((500 \mu \mathrm{L}, 1.0 \mathrm{M}$ in THF, $0.5 \mathrm{mmol})$ in a flame dried 2neck round-bottomed flask, equipped with a stir bar and a septum under argon. Dry THF (0.7 $\mathrm{mL}$ ) was added and the clear, slightly-yellow solution was immersed into a water-bath at $23{ }^{\circ} \mathrm{C} \pm$ $1{ }^{\circ} \mathrm{C}$ for $30 \mathrm{~min}$. The 2-iodothiophene $(28 \mu \mathrm{L}, 0.25 \mathrm{mmol})$ was then added and the reaction was stirred for $1 \mathrm{~min}$. A suspension of $\operatorname{Pd}(\mathrm{dba})_{2}(7.2 \mathrm{mg}, 0.0125 \mathrm{mmol})$ in dry THF $(1.0 \mathrm{~mL})$ was added. The initially purple, turbid reaction mixture turns into a clear solution within 30 seconds. Aliquots $(\sim 200 \mu \mathrm{L}$ )were withdrawn after 1, 2, 3, 4, 5, 7, 10, 15, 20, 30, 40, 50, 60, 75, 90, and $120 \mathrm{~min}$. To quench, these samples were added to $100 \mu \mathrm{L}$ of a $20 \%$ aqueous solution of 2dimethylaminoethanethiol hydrochloride. The clear colorless to slightly-yellow solution was then filtered through a plug of silica gel, and the silica gel was washed with diethyl ether to give a total sample volume of $\sim 2 \mathrm{~mL}$. This sample was injected into the GC three times.

initial rate $=0.0442$

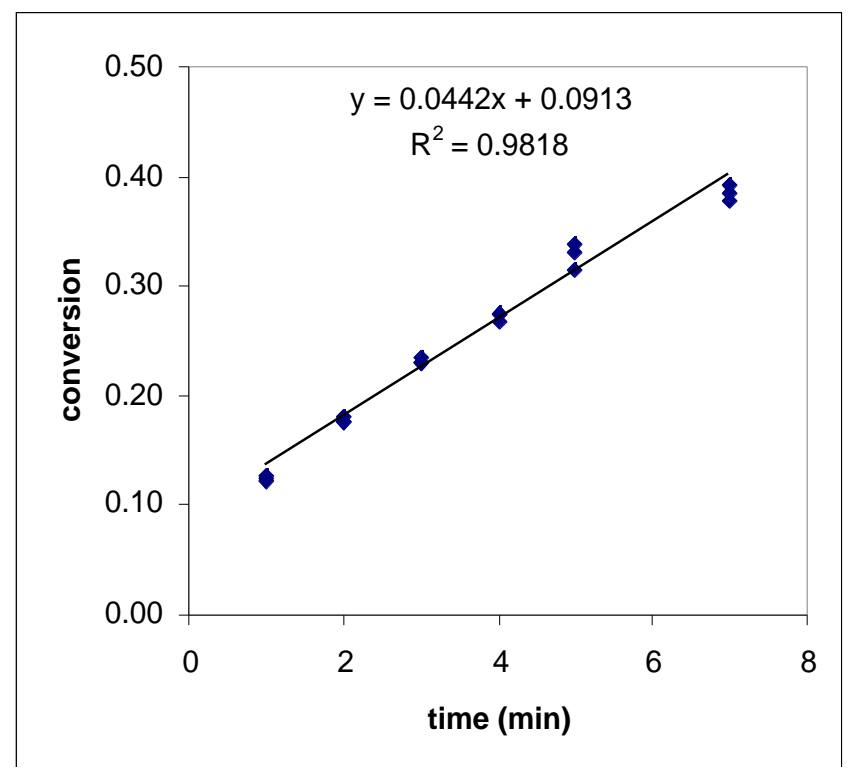




\begin{tabular}{|c|c|c|c|}
\hline time (min) & area $\mathrm{A}$ & area 16 & conversion \\
\hline 1 & 11915 & 1820 & 0.122 \\
\hline 1 & 11992 & 1904 & 0.127 \\
\hline 1 & 12505 & 1928 & 0.123 \\
\hline 2 & 8352 & 1849 & 0.177 \\
\hline 2 & 8235 & 1855 & 0.180 \\
\hline 2 & 7961 & 1755 & 0.176 \\
\hline 3 & 7798 & 2243 & 0.230 \\
\hline 3 & 8007 & 2303 & 0.230 \\
\hline 3 & 7997 & 2358 & 0.236 \\
\hline 4 & 7874 & 2636 & 0.268 \\
\hline 4 & 7398 & 2543 & 0.275 \\
\hline 4 & 7920 & 2722 & 0.275 \\
\hline 5 & 12050 & 4993 & 0.331 \\
\hline 5 & 11358 & 4793 & 0.337 \\
\hline 5 & 10605 & 4178 & 0.315 \\
\hline 7 & 8435 & 4145 & 0.393 \\
\hline 7 & 8554 & 4113 & 0.384 \\
\hline 7 & 7688 & 3642 & 0.379 \\
\hline 10 & 11533 & 6777 & 0.470 \\
\hline 10 & 12117 & 7043 & 0.465 \\
\hline 10 & 11717 & 6845 & 0.467 \\
\hline 15 & 15198 & 11474 & 0.604 \\
\hline 15 & 15485 & 11818 & 0.610 \\
\hline 15 & 15059 & 11370 & 0.604 \\
\hline 20 & 7928 & 7479 & 0.754 \\
\hline 20 & 7628 & 7186 & 0.753 \\
\hline 20 & 8199 & 7727 & 0.753 \\
\hline 30 & 12542 & 16965 & 1.081 \\
\hline 30 & 13311 & 17780 & 1.068 \\
\hline 30 & 12079 & 16162 & 1.070 \\
\hline
\end{tabular}


Order in $(E)-7,(0.25$ equiv).

(E)-7

2-Iodothiophene

Tetra- $n$-butylammonium fluoride

Bis(dibenzylideneacetone)palladium $2.5 \mathrm{mM}$ (0.05 equiv)
$12.5 \mathrm{mM}(0.25$ equiv $)$

$50 \mathrm{mM}$ (1.0 equiv)

$188 \mathrm{mM}$ (3.75 equiv)

Following the General Procedure, disiloxane $(E)-7$ (20 mg, $0.063 \mathrm{mmol})$ and biphenyl $(11.8 \mathrm{mg})$ were dissolved in TBAF solution $(940 \mu \mathrm{L}, 1.0 \mathrm{M}$ in THF, $0.94 \mathrm{mmol})$ in a flame dried 2-neck round-bottomed flask, equipped with a stir bar and a septum under argon. Dry THF (3.05 $\mathrm{mL}$ ) was added and the clear, slightly-yellow solution was immersed into a water-bath at $23{ }^{\circ} \mathrm{C} \pm$ $1{ }^{\circ} \mathrm{C}$ for $30 \mathrm{~min}$. The 2-iodothiophene $(28 \mu \mathrm{L}, 0.25 \mathrm{mmol})$ was then added and the reaction was stirred for $1 \mathrm{~min}$. A suspension of $\mathrm{Pd}(\mathrm{dba})_{2}(7.2 \mathrm{mg}, 0.0125 \mathrm{mmol})$ in dry THF $(1.0 \mathrm{~mL})$ was added. The initially purple, turbid reaction mixture turns into a clear solution within 30 seconds. Aliquots $(\sim 200 \mu \mathrm{L})$ were withdrawn after 1, 2, 3, 4, 5, 7, 10, 15, 20, 30, 40, 50, 60, 75, 90, and $120 \mathrm{~min}$. To quench, these samples were added to $100 \mu \mathrm{L}$ of a $20 \%$ aqueous solution of 2dimethylaminoethanethiol hydrochloride. The clear colorless to slightly-yellow solution was then filtered through a plug of silica gel, and the silica gel was washed with diethyl ether to give a total sample volume of $\sim 2 \mathrm{~mL}$. This sample was injected into the GC three times.

initial rate $=0.0056$

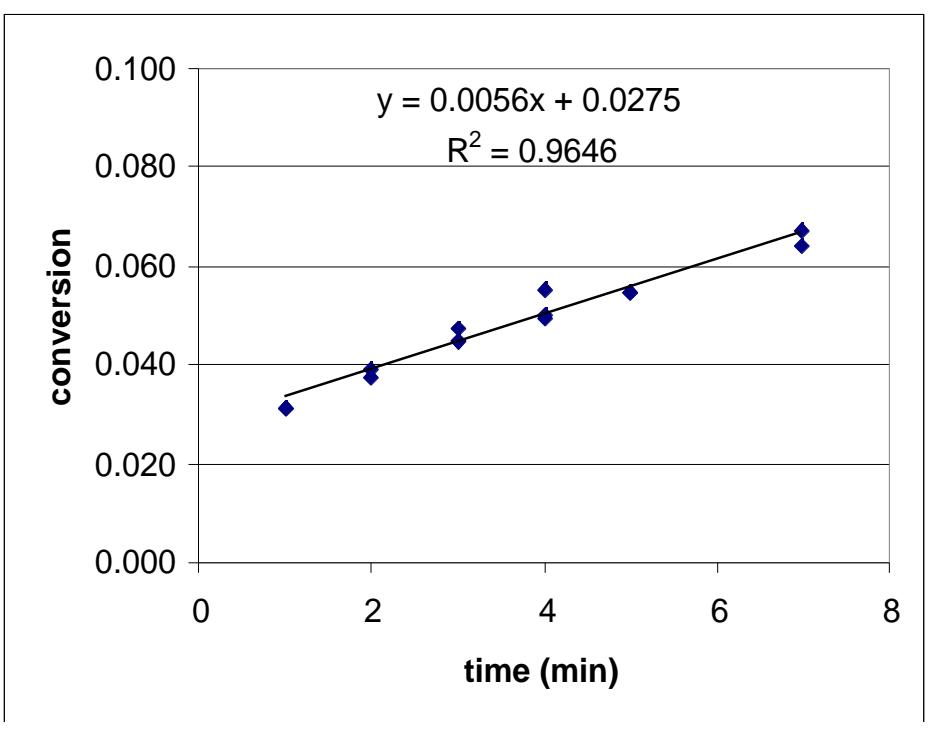




\begin{tabular}{|c|c|c|c|}
\hline Time & area standard & area 16 & conversion \\
\hline 1 & 9502 & 668 & 0.031 \\
\hline 1 & 10172 & 715 & 0.031 \\
\hline 2 & 9342 & 816 & 0.039 \\
\hline 2 & 10371 & 876 & 0.038 \\
\hline 3 & 4166 & 443 & 0.047 \\
\hline 3 & 9541 & 957 & 0.045 \\
\hline 3 & 10488 & 1054 & 0.045 \\
\hline 4 & 3413 & 425 & 0.055 \\
\hline 4 & 8063 & 909 & 0.050 \\
\hline 4 & 8694 & 967 & 0.049 \\
\hline 5 & 9697 & 1197 & 0.055 \\
\hline 5 & 9701 & 1192 & 0.055 \\
\hline 7 & 5961 & 897 & 0.067 \\
\hline 7 & 6414 & 926 & 0.064 \\
\hline 10 & 3378 & 588 & 0.077 \\
\hline 10 & 4313 & 762 & 0.079 \\
\hline 10 & 4110 & 720 & 0.078 \\
\hline 15 & 3778 & 804 & 0.095 \\
\hline 15 & 3958 & 815 & 0.092 \\
\hline 20 & 2150 & 497 & 0.103 \\
\hline 20 & 3288 & 753 & 0.102 \\
\hline 20 & 3408 & 808 & 0.106 \\
\hline 30 & 4919 & 1629 & 0.147 \\
\hline 30 & 5129 & 1505 & 0.131 \\
\hline 30 & 5247 & 1524 & 0.129 \\
\hline 40 & 3776 & 1287 & 0.152 \\
\hline 40 & 4355 & 1505 & 0.154 \\
\hline 40 & 4531 & 1501 & 0.147 \\
\hline 50 & 4373 & 1726 & 0.176 \\
\hline 50 & 4978 & 1961 & 0.175 \\
\hline 50 & 5316 & 2041 & 0.171 \\
\hline 60 & 5165 & 2213 & 0.191 \\
\hline 60 & 5429 & 2228 & 0.183 \\
\hline 60 & 5430 & 2357 & 0.193 \\
\hline 75 & 4435 & 2125 & 0.213 \\
\hline 75 & 4766 & 2265 & 0.211 \\
\hline 75 & 4623 & 2179 & 0.210 \\
\hline 90 & 4331 & 2314 & 0.238 \\
\hline 90 & 4317 & 2228 & 0.230 \\
\hline 90 & 4253 & 2221 & 0.232 \\
\hline 120 & 4210 & 2514 & 0.266 \\
\hline 120 & 4528 & 2830 & 0.278 \\
\hline 120 & 4558 & 2805 & 0.274 \\
\hline
\end{tabular}




\section{Order in $(E)-7$, (0.5 equiv).}

\section{$(E)-7$}

2-Iodothiophene

Tetra- $n$-butylammonium fluoride

Bis(dibenzylideneacetone)palladium $2.5 \mathrm{mM}$ (0.05 equiv)
$25 \mathrm{mM}(0.5$ equiv $)$

$50 \mathrm{mM}$ (1.0 equiv)

$188 \mathrm{mM}$ (3.75 equiv)

Following the General Procedure, disiloxane $(E)-7(41 \mathrm{mg}, 0.25 \mathrm{mmol})$ and biphenyl $(11.0 \mathrm{mg})$ were dissolved in TBAF solution $(940 \mu \mathrm{L}, 1.0 \mathrm{M}$ in THF, $0.94 \mathrm{mmol})$ in a flame dried 2-neck round-bottomed flask, equipped with a stir bar and a septum under argon. Dry THF (3.00 $\mathrm{mL}$ ) was added and the clear, slightly-yellow solution was immersed into a water-bath at $23{ }^{\circ} \mathrm{C} \pm$ $1{ }^{\circ} \mathrm{C}$ for $30 \mathrm{~min}$. The 2-iodothiophene $(28 \mu \mathrm{L}, 0.25 \mathrm{mmol})$ was then added and the reaction was stirred for $1 \mathrm{~min}$. A suspension of $\mathrm{Pd}(\mathrm{dba})_{2}(7.2 \mathrm{mg}, 0.0125 \mathrm{mmol})$ in dry THF $(1.0 \mathrm{~mL})$ was added. The initially purple, turbid reaction mixture turns into a clear solution within 30 seconds. Aliquots $(\sim 200 \mu \mathrm{L}$ )were withdrawn after 1, 2, 3, 4, 5, 7, 10, 15, 20, 30, 40, 50, 60, 75, 90, and $120 \mathrm{~min}$. To quench, these samples were added to $100 \mu \mathrm{L}$ of a $20 \%$ aqueous solution of 2dimethylaminoethanethiol hydrochloride. The clear colorless to slightly-yellow solution was then filtered through a plug of silica gel, and the silica gel was washed with diethyl ether to give a total sample volume of $\sim 2 \mathrm{~mL}$. This sample was injected into the GC three times.

initial rate $=0.0124$

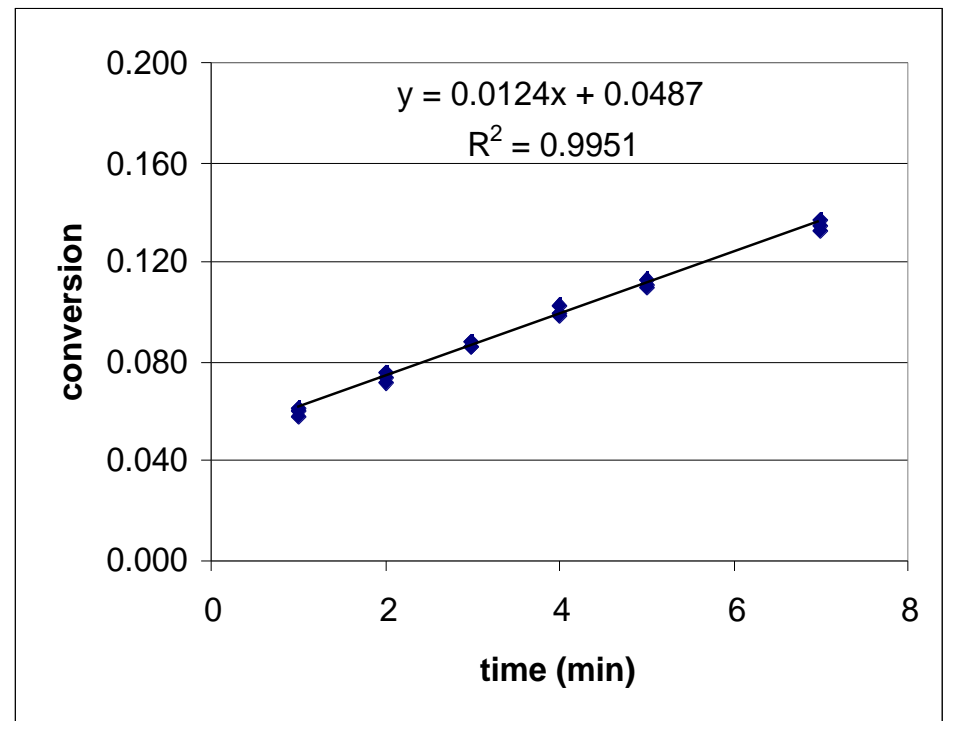




\begin{tabular}{|c|c|c|c|}
\hline time & area standard & area 16 & conversion \\
\hline 1 & 4821 & 695 & 0.060 \\
\hline 1 & 4499 & 659 & 0.061 \\
\hline 1 & 5143 & 724 & 0.058 \\
\hline 2 & 4883 & 893 & 0.076 \\
\hline 2 & 4720 & 819 & 0.072 \\
\hline 2 & 4825 & 852 & 0.073 \\
\hline 3 & 5534 & 1153 & 0.086 \\
\hline 3 & 5356 & 1104 & 0.086 \\
\hline 3 & 5584 & 1182 & 0.088 \\
\hline 4 & 4447 & 1063 & 0.099 \\
\hline 4 & 4373 & 1076 & 0.102 \\
\hline 4 & 4708 & 1122 & 0.099 \\
\hline 5 & 4707 & 1254 & 0.111 \\
\hline 5 & 4302 & 1166 & 0.112 \\
\hline 5 & 4647 & 1232 & 0.110 \\
\hline 7 & 4508 & 1436 & 0.132 \\
\hline 7 & 4088 & 1346 & 0.137 \\
\hline 7 & 4448 & 1443 & 0.135 \\
\hline 10 & 4461 & 1733 & 0.161 \\
\hline 10 & 4494 & 1720 & 0.159 \\
\hline 10 & 4671 & 1793 & 0.159 \\
\hline 15 & 4398 & 2195 & 0.207 \\
\hline 15 & 4051 & 2186 & 0.224 \\
\hline 15 & 4564 & 2288 & 0.208 \\
\hline 20 & 5126 & 2990 & 0.242 \\
\hline 20 & 5138 & 3024 & 0.244 \\
\hline 20 & 5133 & 2928 & 0.237 \\
\hline 30 & 4366 & 3284 & 0.312 \\
\hline 30 & 4585 & 3333 & 0.302 \\
\hline 30 & 5060 & 3748 & 0.307 \\
\hline 40 & 5028 & 4528 & 0.374 \\
\hline 40 & 4879 & 4393 & 0.374 \\
\hline 40 & 5061 & 4631 & 0.380 \\
\hline 50 & 4289 & 4525 & 0.438 \\
\hline 50 & 4356 & 4618 & 0.440 \\
\hline 50 & 4689 & 4970 & 0.440 \\
\hline 60 & 5455 & 6484 & 0.493 \\
\hline 60 & 4906 & 5998 & 0.507 \\
\hline 60 & 5872 & 6856 & 0.484 \\
\hline 75 & 4987 & 6616 & 0.550 \\
\hline 75 & 4928 & 6607 & 0.556 \\
\hline 75 & 5060 & 6792 & 0.557 \\
\hline 90 & 5328 & 7877 & 0.613 \\
\hline 90 & 5108 & 7715 & 0.627 \\
\hline 90 & 5748 & 8571 & 0.619 \\
\hline 120 & 4615 & 7882 & 0.709 \\
\hline
\end{tabular}




\section{Order in $(E)-7$, (1.0 equiv).}

\section{$(E)-7$}

2-Iodothiophene

Tetra- $n$-butylammonium fluoride

Bis(dibenzylideneacetone)palladium $2.5 \mathrm{mM}$ (0.05 equiv)
$50 \mathrm{mM}$ (1.0 equiv)

$50 \mathrm{mM}$ (1.0 equiv)

$188 \mathrm{mM}$ (3.75 equiv)

Following the General Procedure, disiloxane $(E)-7(82 \mathrm{mg}, 0.25 \mathrm{mmol})$ and biphenyl (11.4 mg) were dissolved in TBAF solution $(940 \mu \mathrm{L}, 1.0 \mathrm{M}$ in THF, $0.94 \mathrm{mmol})$ in a flame dried 2-neck round-bottomed flask, equipped with a stir bar and a septum under argon. Dry THF (2.95 $\mathrm{mL}$ ) was added and the clear, slightly-yellow solution was immersed into a water-bath at $23{ }^{\circ} \mathrm{C} \pm$ $1{ }^{\circ} \mathrm{C}$ for $30 \mathrm{~min}$. The 2-iodothiophene $(28 \mu \mathrm{L}, 0.25 \mathrm{mmol})$ was then added and the reaction was stirred for $1 \mathrm{~min}$. A suspension of $\operatorname{Pd}(\mathrm{dba})_{2}(7.2 \mathrm{mg}, 0.0125 \mathrm{mmol})$ in dry THF $(1.0 \mathrm{~mL})$ was added. The initially purple, turbid reaction mixture turns into a clear solution within 30 seconds. Aliquots $(\sim 200 \mu \mathrm{L}$ )were withdrawn after 1, 2, 3, 4, 5, 7, 10, 15, 20, 30, 40, 50, 60, 75, 90, and $120 \mathrm{~min}$. To quench, these samples were added to $100 \mu \mathrm{L}$ of a $20 \%$ aqueous solution of 2dimethylaminoethanethiol hydrochloride. The clear colorless to slightly-yellow solution was then filtered through a plug of silica gel, and the silica gel was washed with diethyl ether to give a total sample volume of $\sim 2 \mathrm{~mL}$. This sample was injected into the GC three times.

initial rate $=0.0214$

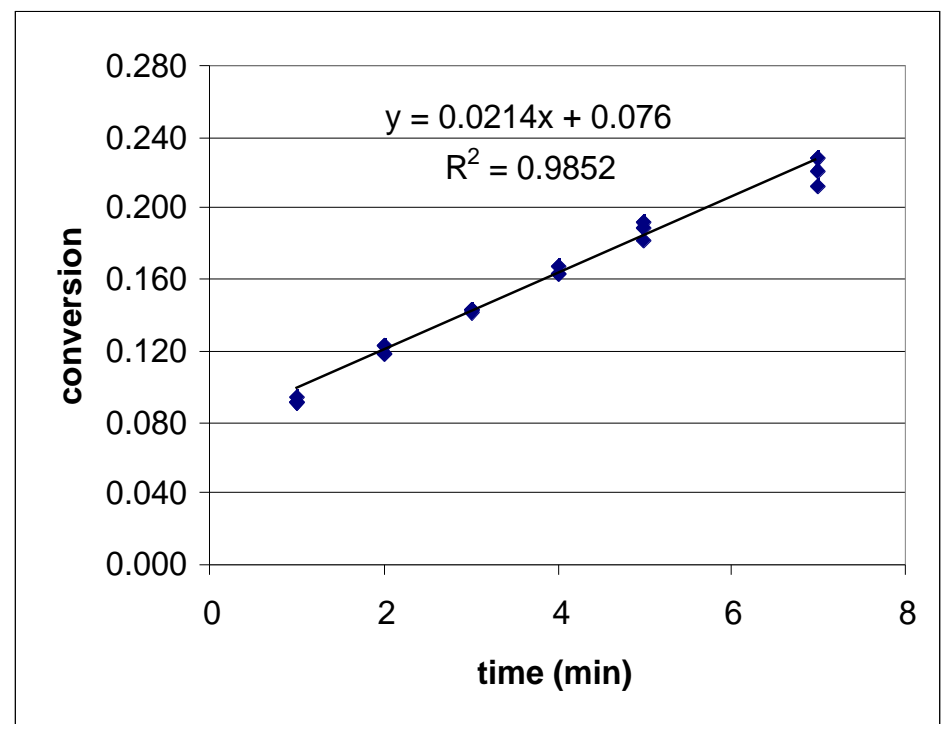




\begin{tabular}{|c|c|c|c|}
\hline time & area standard & area 16 & conversion \\
\hline 1 & 3815 & 833 & 0.047 \\
\hline 1 & 3883 & 826 & 0.046 \\
\hline 1 & 4597 & 976 & 0.046 \\
\hline 2 & 6094 & 1680 & 0.059 \\
\hline 2 & 5950 & 1694 & 0.061 \\
\hline 2 & 7111 & 1963 & 0.059 \\
\hline 3 & 6109 & 2035 & 0.072 \\
\hline 3 & 5951 & 1957 & 0.071 \\
\hline 3 & 6890 & 2300 & 0.072 \\
\hline 4 & 6300 & 2400 & 0.082 \\
\hline 4 & 6286 & 2445 & 0.084 \\
\hline 4 & 7181 & 2724 & 0.082 \\
\hline 5 & 4375 & 1849 & 0.091 \\
\hline 5 & 4432 & 1979 & 0.096 \\
\hline 5 & 4941 & 2179 & 0.095 \\
\hline 7 & 4698 & 2417 & 0.111 \\
\hline 7 & 4832 & 2390 & 0.106 \\
\hline 7 & 5278 & 2806 & 0.114 \\
\hline 10 & 3819 & 2437 & 0.137 \\
\hline 10 & 4036 & 2565 & 0.137 \\
\hline 10 & 4323 & 2776 & 0.138 \\
\hline 15 & 5507 & 4482 & 0.175 \\
\hline 15 & 5779 & 4693 & 0.175 \\
\hline 15 & 6253 & 5015 & 0.172 \\
\hline 20 & 4488 & 4320 & 0.207 \\
\hline 20 & 4529 & 4348 & 0.206 \\
\hline 20 & 4855 & 4711 & 0.209 \\
\hline 30 & 5238 & 6665 & 0.274 \\
\hline 30 & 5500 & 6924 & 0.271 \\
\hline 30 & 5994 & 7575 & 0.272 \\
\hline 40 & 5551 & 8429 & 0.326 \\
\hline 40 & 5563 & 8643 & 0.334 \\
\hline 40 & 6496 & 9794 & 0.324 \\
\hline 50 & 5679 & 9915 & 0.375 \\
\hline 50 & 6023 & 10553 & 0.377 \\
\hline 50 & 6083 & 10704 & 0.378 \\
\hline 60 & 5502 & 10573 & 0.413 \\
\hline 60 & 5476 & 10659 & 0.418 \\
\hline 60 & 5694 & 10997 & 0.415 \\
\hline 75 & 6239 & 13739 & 0.473 \\
\hline 75 & 6419 & 14121 & 0.473 \\
\hline 75 & 6625 & 14463 & 0.469 \\
\hline 90 & 5407 & 12959 & 0.515 \\
\hline 90 & 5629 & 13598 & 0.519 \\
\hline 90 & 6169 & 14990 & 0.522 \\
\hline 120 & 6417 & 16322 & 0.547 \\
\hline 120 & 6227 & 15858 & 0.547 \\
\hline
\end{tabular}




\section{Order in TBAF, (1.0 equiv).}

(E)-1

2-Iodothiophene

Tetra- $n$-butylammonium fluoride

Bis(dibenzylideneacetone)palladium $2.5 \mathrm{mM}$ (0.05 equiv)
$50 \mathrm{mM}$ (1.0 equiv)

$50 \mathrm{mM}$ (1.0 equiv)

$50 \mathrm{mM}(1.0$ equiv)

Following the General Procedure, silanol (E)-1 (43 mg, $0.25 \mathrm{mmol})$ and biphenyl (6.3 $\mathrm{mg}$ ) were dissolved in TBAF solution $(250 \mu \mathrm{L}, 1.0 \mathrm{M}$ in THF, $0.25 \mathrm{mmol})$ in a flame dried 2neck round-bottomed flask, equipped with a stir bar and a septum under argon. Dry THF (3.75 $\mathrm{mL}$ ) was added and the clear, slightly-yellow solution was immersed into a water-bath at $23{ }^{\circ} \mathrm{C} \pm$ $1{ }^{\circ} \mathrm{C}$ for $30 \mathrm{~min}$. The 2-iodothiophene $(28 \mu \mathrm{L}, 0.25 \mathrm{mmol})$ was then added and the reaction was stirred for $1 \mathrm{~min}$. A suspension of $\operatorname{Pd}(\mathrm{dba})_{2}(7.2 \mathrm{mg}, 0.0125 \mathrm{mmol})$ in dry THF $(1.0 \mathrm{~mL})$ was added. The initially purple, turbid reaction mixture turns into a clear solution within 30 seconds. Aliquots $(\sim 200 \mu \mathrm{L}$ )were withdrawn after 1, 2, 3, 4, 5, 7, 10, 15, 20, 30, 40, 50, 60, 75, 90, and $120 \mathrm{~min}$. To quench, these samples were added to $100 \mu \mathrm{L}$ of a $20 \%$ aqueous solution of 2dimethylaminoethanethiol hydrochloride. The clear colorless to slightly-yellow solution was then filtered through a plug of silica gel, and the silica gel was washed with diethyl ether to give a total sample volume of $\sim 2 \mathrm{~mL}$. This sample was injected into the GC three times.

initial rate $=0.0085$

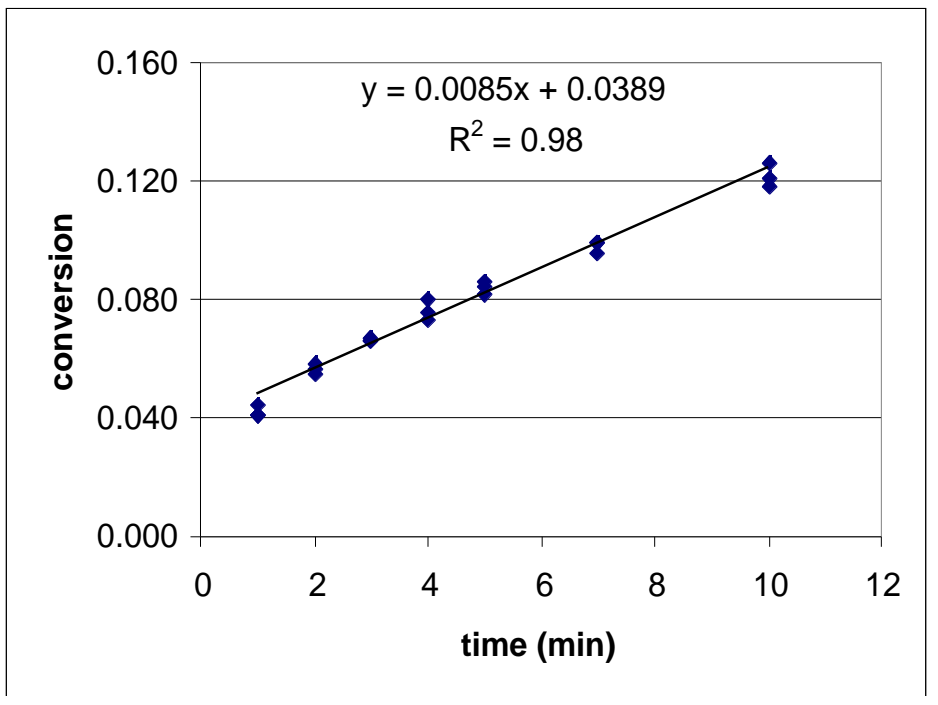




\begin{tabular}{|c|c|c|c|}
\hline time & area $\mathrm{A}$ & area 16 & conversion \\
\hline 1 & 5186 & 961 & 0.044 \\
\hline 1 & 4372 & 749 & 0.041 \\
\hline 1 & 7902 & 1366 & 0.041 \\
\hline 2 & 6542 & 1560 & 0.057 \\
\hline 2 & 5787 & 1336 & 0.055 \\
\hline 2 & 7329 & 1797 & 0.058 \\
\hline 3 & 5944 & 1645 & 0.066 \\
\hline 3 & 5736 & 1620 & 0.067 \\
\hline 3 & 6386 & 1780 & 0.066 \\
\hline 4 & 4806 & 1613 & 0.080 \\
\hline 4 & 6041 & 1934 & 0.076 \\
\hline 4 & 5965 & 1829 & 0.073 \\
\hline 5 & 5542 & 2013 & 0.086 \\
\hline 5 & 5376 & 1917 & 0.085 \\
\hline 5 & 5700 & 1961 & 0.082 \\
\hline 7 & 5230 & 2105 & 0.096 \\
\hline 7 & 5230 & 2185 & 0.099 \\
\hline 7 & 6790 & 2837 & 0.099 \\
\hline 10 & 5030 & 2661 & 0.126 \\
\hline 10 & 5203 & 2653 & 0.121 \\
\hline 10 & 5858 & 2921 & 0.118 \\
\hline 15 & 4957 & 3267 & 0.157 \\
\hline 15 & 5455 & 3448 & 0.150 \\
\hline 15 & 5561 & 3417 & 0.146 \\
\hline 20 & 5945 & 4233 & 0.169 \\
\hline 20 & 6386 & 4558 & 0.170 \\
\hline 20 & 8482 & 5925 & 0.166 \\
\hline 30 & 6945 & 5944 & 0.203 \\
\hline 30 & 7027 & 5891 & 0.199 \\
\hline 30 & 8928 & 7472 & 0.199 \\
\hline 40 & 5676 & 5569 & 0.233 \\
\hline 40 & 6455 & 6190 & 0.228 \\
\hline 40 & 10521 & 10085 & 0.228 \\
\hline 50 & 5548 & 5862 & 0.251 \\
\hline 50 & 5865 & 6239 & 0.253 \\
\hline 50 & 6761 & 7094 & 0.249 \\
\hline 60 & 5355 & 6058 & 0.269 \\
\hline 60 & 5591 & 6376 & 0.271 \\
\hline 60 & 6332 & 7285 & 0.273 \\
\hline 75 & 6192 & 7776 & 0.298 \\
\hline 75 & 6256 & 7789 & 0.296 \\
\hline 75 & 7886 & 10014 & 0.302 \\
\hline 90 & 4473 & 5990 & 0.318 \\
\hline 90 & 4725 & 6465 & 0.325 \\
\hline 120 & 6223 & 9481 & 0.362 \\
\hline 120 & 5968 & 9084 & 0.362 \\
\hline
\end{tabular}




\section{Order in TBAF, (1.0 equiv).}

(E)-1

2-Iodothiophene

Tetra- $n$-butylammonium fluoride

Bis(dibenzylideneacetone)palladium $2.5 \mathrm{mM}$ (0.05 equiv)
$50 \mathrm{mM}$ (1.0 equiv)

$50 \mathrm{mM}$ (1.0 equiv)

$50 \mathrm{mM}$ (1.0 equiv)

Following the General Procedure, silanol (E)-1 (43 mg, $0.25 \mathrm{mmol})$ and biphenyl $(7.4$ $\mathrm{mg}$ ) were dissolved in TBAF solution $(250 \mu \mathrm{L}, 1.0 \mathrm{M}$ in THF, $0.25 \mathrm{mmol})$ in a flame dried 2neck round-bottomed flask, equipped with a stir bar and a septum under argon. Dry THF (3.75 $\mathrm{mL}$ ) was added and the clear, slightly-yellow solution was immersed into a water-bath at $23{ }^{\circ} \mathrm{C} \pm$ $1{ }^{\circ} \mathrm{C}$ for $30 \mathrm{~min}$. The 2-iodothiophene $(28 \mu \mathrm{L}, 0.25 \mathrm{mmol})$ was then added and the reaction was stirred for $1 \mathrm{~min}$. A suspension of $\operatorname{Pd}(\mathrm{dba})_{2}(7.2 \mathrm{mg}, 0.0125 \mathrm{mmol})$ in dry THF $(1.0 \mathrm{~mL})$ was added. The initially purple, turbid reaction mixture turns into a clear solution within 30 seconds. Aliquots $(\sim 200 \mu \mathrm{L}$ )were withdrawn after 1, 2, 3, 4, 5, 7, 10, 15, 20, 30, 40, 50, 60, 75, 90, and $120 \mathrm{~min}$. To quench, these samples were added to $100 \mu \mathrm{L}$ of a $20 \%$ aqueous solution of 2dimethylaminoethanethiol hydrochloride. The clear colorless to slightly-yellow solution was then filtered through a plug of silica gel, and the silica gel was washed with diethyl ether to give a total sample volume of $\sim 2 \mathrm{~mL}$. This sample was injected into the GC three times.

initial rate $=0.0107$

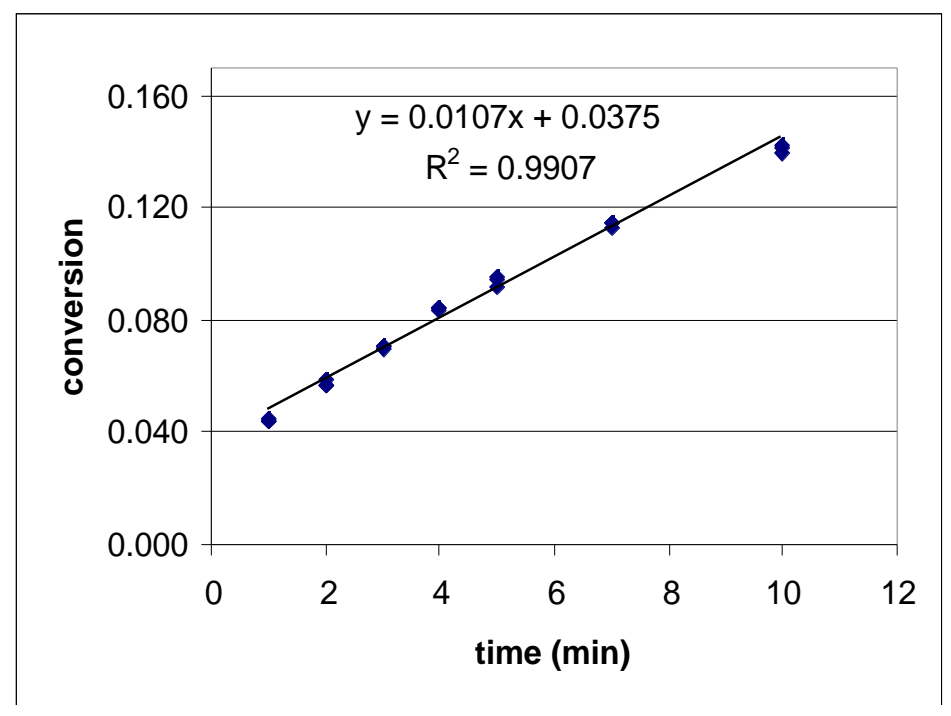




\begin{tabular}{|c|c|c|c|}
\hline time & area $\mathrm{A}$ & area 16 & conversion \\
\hline 1 & 6382 & 1023 & 0.045 \\
\hline 1 & 10621 & 1673 & 0.044 \\
\hline 1 & 10399 & 1635 & 0.044 \\
\hline 2 & 6718 & 1409 & 0.059 \\
\hline 2 & 10843 & 2210 & 0.057 \\
\hline 2 & 12468 & 2555 & 0.057 \\
\hline 3 & 6510 & 1656 & 0.071 \\
\hline 3 & 8352 & 2107 & 0.070 \\
\hline 3 & 8483 & 2129 & 0.070 \\
\hline 4 & 6772 & 2055 & 0.085 \\
\hline 4 & 8884 & 2655 & 0.083 \\
\hline 4 & 9967 & 3005 & 0.084 \\
\hline 5 & 6070 & 2001 & 0.092 \\
\hline 5 & 8017 & 2731 & 0.095 \\
\hline 5 & 9792 & 3340 & 0.095 \\
\hline 7 & 6378 & 2634 & 0.115 \\
\hline 7 & 9417 & 3868 & 0.115 \\
\hline 7 & 10487 & 4239 & 0.113 \\
\hline 10 & 6269 & 3190 & 0.142 \\
\hline 10 & 10257 & 5187 & 0.141 \\
\hline 10 & 10613 & 5326 & 0.140 \\
\hline 15 & 5503 & 3345 & 0.170 \\
\hline 15 & 6215 & 3758 & 0.169 \\
\hline 15 & 7034 & 4114 & 0.163 \\
\hline 20 & 6472 & 4535 & 0.196 \\
\hline 20 & 8178 & 5677 & 0.194 \\
\hline 20 & 7551 & 5214 & 0.193 \\
\hline 30 & 8052 & 6858 & 0.238 \\
\hline 30 & 9851 & 8167 & 0.231 \\
\hline 30 & 10786 & 8125 & 0.210 \\
\hline 40 & 5256 & 5022 & 0.267 \\
\hline 40 & 12004 & 11304 & 0.263 \\
\hline 40 & 13839 & 13078 & 0.264 \\
\hline 50 & 5598 & 5918 & 0.295 \\
\hline 50 & 15229 & 16091 & 0.295 \\
\hline 50 & 19796 & 20597 & 0.290 \\
\hline 60 & 5616 & 6322 & 0.314 \\
\hline 60 & 8022 & 8944 & 0.311 \\
\hline 60 & 8308 & 9443 & 0.317 \\
\hline 75 & 6994 & 8647 & 0.345 \\
\hline 75 & 13350 & 16343 & 0.342 \\
\hline 75 & 13530 & 16508 & 0.341 \\
\hline 90 & 5585 & 7333 & 0.366 \\
\hline 90 & 7814 & 10319 & 0.369 \\
\hline 90 & 7625 & 9967 & 0.365 \\
\hline 120 & 5768 & 8255 & 0.399 \\
\hline 120 & 8271 & 11888 & 0.401 \\
\hline
\end{tabular}




\section{Order in TBAF, (1.0 equiv).}

(E)-1

2-Iodothiophene

Tetra- $n$-butylammonium fluoride

Bis(dibenzylideneacetone)palladium $2.5 \mathrm{mM}$ (0.05 equiv)
$50 \mathrm{mM}$ (1.0 equiv)

$50 \mathrm{mM}$ (1.0 equiv)

$50 \mathrm{mM}$ (1.0 equiv)

Following the General Procedure, silanol (E)-1 (43 mg, $0.25 \mathrm{mmol})$ and biphenyl $(7.9$ $\mathrm{mg}$ ) were dissolved in TBAF solution $(250 \mu \mathrm{L}, 1.0 \mathrm{M}$ in THF, $0.25 \mathrm{mmol})$ in a flame dried 2neck round-bottomed flask, equipped with a stir bar and a septum under argon. Dry THF (3.75 $\mathrm{mL}$ ) was added and the clear, slightly-yellow solution was immersed into a water-bath at $23{ }^{\circ} \mathrm{C} \pm$ $1{ }^{\circ} \mathrm{C}$ for $30 \mathrm{~min}$. The 2-iodothiophene $(28 \mu \mathrm{L}, 0.25 \mathrm{mmol})$ was then added and the reaction was stirred for $1 \mathrm{~min}$. A suspension of $\operatorname{Pd}(\mathrm{dba})_{2}(7.2 \mathrm{mg}, 0.0125 \mathrm{mmol})$ in dry THF $(1.0 \mathrm{~mL})$ was added. The initially purple, turbid reaction mixture turns into a clear solution within 30 seconds. Aliquots $(\sim 200 \mu \mathrm{L}$ )were withdrawn after 1, 2, 3, 4, 5, 7, 10, 15, 20, 30, 40, 50, 60, 75, 90, and $120 \mathrm{~min}$. To quench, these samples were added to $100 \mu \mathrm{L}$ of a $20 \%$ aqueous solution of 2dimethylaminoethanethiol hydrochloride. The clear colorless to slightly-yellow solution was then filtered through a plug of silica gel, and the silica gel was washed with diethyl ether to give a total sample volume of $\sim 2 \mathrm{~mL}$. This sample was injected into the GC three times.

initial rate $=0.0081$

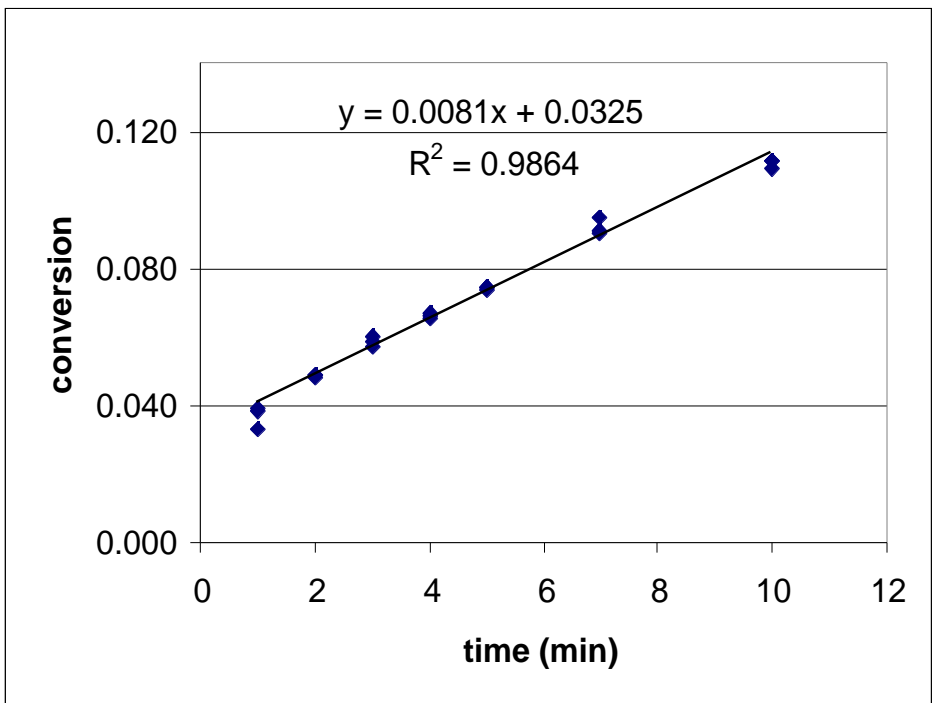




\begin{tabular}{|c|c|c|c|}
\hline time & area $\mathrm{A}$ & area 16 & conversion \\
\hline 1 & 7184 & 797 & 0.033 \\
\hline 1 & 8692 & 1117 & 0.038 \\
\hline 1 & 10116 & 1327 & 0.039 \\
\hline 2 & 7053 & 1166 & 0.049 \\
\hline 2 & 7930 & 1295 & 0.049 \\
\hline 2 & 8561 & 1383 & 0.048 \\
\hline 3 & 8155 & 1646 & 0.060 \\
\hline 3 & 10579 & 2086 & 0.059 \\
\hline 3 & 11084 & 2139 & 0.057 \\
\hline 4 & 6904 & 1557 & 0.067 \\
\hline 4 & 11119 & 2456 & 0.066 \\
\hline 4 & 9754 & 2162 & 0.066 \\
\hline 5 & 7757 & 1931 & 0.074 \\
\hline 5 & 14450 & 3613 & 0.074 \\
\hline 5 & 13884 & 3440 & 0.074 \\
\hline 7 & 7478 & 2377 & 0.095 \\
\hline 7 & 14324 & 4334 & 0.090 \\
\hline 7 & 12811 & 3905 & 0.091 \\
\hline 10 & 7402 & 2764 & 0.111 \\
\hline 10 & 7410 & 2710 & 0.109 \\
\hline 10 & 11682 & 4363 & 0.111 \\
\hline 15 & 6321 & 2958 & 0.139 \\
\hline 15 & 7512 & 3492 & 0.138 \\
\hline 15 & 11295 & 5300 & 0.140 \\
\hline 20 & 6523 & 3577 & 0.163 \\
\hline 20 & 7330 & 3994 & 0.162 \\
\hline 20 & 8925 & 4625 & 0.154 \\
\hline 30 & 7404 & 4828 & 0.194 \\
\hline 30 & 9370 & 5970 & 0.190 \\
\hline 30 & 12561 & 7962 & 0.189 \\
\hline 40 & 6835 & 5112 & 0.223 \\
\hline 40 & 11391 & 8311 & 0.217 \\
\hline 40 & 13561 & 9860 & 0.217 \\
\hline 50 & 7634 & 6361 & 0.248 \\
\hline 50 & 10016 & 8215 & 0.244 \\
\hline 50 & 12951 & 10465 & 0.241 \\
\hline 60 & 6729 & 5987 & 0.265 \\
\hline 60 & 10926 & 9685 & 0.264 \\
\hline 60 & 15688 & 13595 & 0.258 \\
\hline 75 & 6727 & 6442 & 0.285 \\
\hline 75 & 13873 & 13189 & 0.283 \\
\hline 75 & 16110 & 15329 & 0.283 \\
\hline 90 & 10016 & 10335 & 0.307 \\
\hline 90 & 11286 & 11609 & 0.306 \\
\hline 120 & 7255 & 8426 & 0.346 \\
\hline 120 & 11587 & 13147 & 0.338 \\
\hline
\end{tabular}




\section{Order in TBAF, (1.5 equiv).}

(E)-1

2-Iodothiophene

Tetra- $n$-butylammonium fluoride

Bis(dibenzylideneacetone)palladium $2.5 \mathrm{mM}$ (0.05 equiv)
$50 \mathrm{mM}$ (1.0 equiv)

$50 \mathrm{mM}$ (1.0 equiv)

$75 \mathrm{mM}$ (1.5 equiv)

Following the General Procedure, silanol $(E)-1$ (43 mg, $0.25 \mathrm{mmol})$ and biphenyl (16.5 $\mathrm{mg}$ ) were dissolved in TBAF solution $(375 \mu \mathrm{L}, 1.0 \mathrm{M}$ in THF, $0.375 \mathrm{mmol})$ in a flame dried 2neck round-bottomed flask, equipped with a stir bar and a septum under argon. Dry THF (3.60 $\mathrm{mL}$ ) was added and the clear, slightly-yellow solution was immersed into a water-bath at $23{ }^{\circ} \mathrm{C} \pm$ $1{ }^{\circ} \mathrm{C}$ for $30 \mathrm{~min}$. The 2-iodothiophene $(28 \mu \mathrm{L}, 0.25 \mathrm{mmol})$ was then added and the reaction was stirred for $1 \mathrm{~min}$. A suspension of $\operatorname{Pd}(\mathrm{dba})_{2}(7.2 \mathrm{mg}, 0.0125 \mathrm{mmol})$ in dry THF $(1.0 \mathrm{~mL})$ was added. The initially purple, turbid reaction mixture turns into a clear solution within 30 seconds. Aliquots $(\sim 200 \mu \mathrm{L}$ )were withdrawn after 1, 2, 3, 4, 5, 7, 10, 15, 20, 30, 40, 50, 60, 75, 90, and $120 \mathrm{~min}$. To quench, these samples were added to $100 \mu \mathrm{L}$ of a $20 \%$ aqueous solution of 2dimethylaminoethanethiol hydrochloride. The clear colorless to slightly-yellow solution was then filtered through a plug of silica gel, and the silica gel was washed with diethyl ether to give a total sample volume of $\sim 2 \mathrm{~mL}$. This sample was injected into the $\mathrm{GC}$ three times.

initial rate $=0.0135$

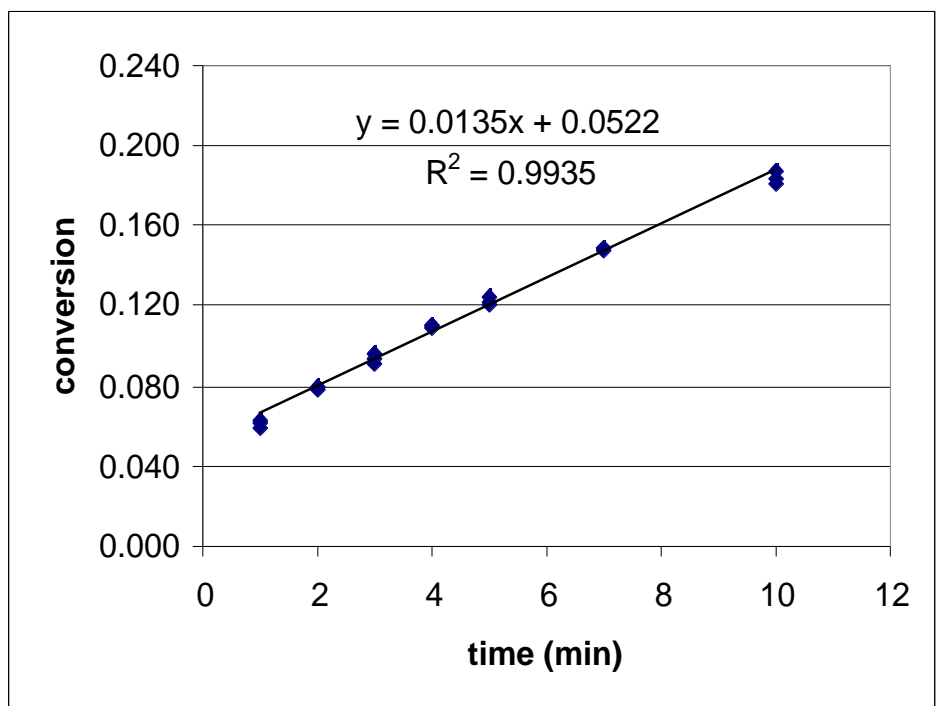




\begin{tabular}{|c|c|c|c|}
\hline Time & area $\mathrm{A}$ & area $\mathbf{1 6}$ & conversion \\
\hline 1 & 13163 & 1317 & 0.062 \\
\hline 1 & 13456 & 1329 & 0.061 \\
\hline 1 & 15514 & 1487 & 0.060 \\
\hline 2 & 16303 & 2079 & 0.079 \\
\hline 2 & 14876 & 1876 & 0.078 \\
\hline 2 & 18429 & 2340 & 0.079 \\
\hline 3 & 14763 & 2158 & 0.091 \\
\hline 3 & 12962 & 1950 & 0.094 \\
\hline 3 & 14495 & 2234 & 0.096 \\
\hline 4 & 11164 & 1972 & 0.110 \\
\hline 4 & 11557 & 2017 & 0.109 \\
\hline 4 & 12673 & 2241 & 0.110 \\
\hline 5 & 12255 & 2411 & 0.122 \\
\hline 5 & 12182 & 2430 & 0.124 \\
\hline 5 & 11654 & 2263 & 0.121 \\
\hline 7 & 15066 & 3602 & 0.149 \\
\hline 7 & 13534 & 3218 & 0.148 \\
\hline 7 & 12412 & 2961 & 0.148 \\
\hline 10 & 12722 & 3705 & 0.181 \\
\hline 10 & 12793 & 3852 & 0.187 \\
\hline 10 & 11263 & 3313 & 0.183 \\
\hline 15 & 10297 & 3948 & 0.239 \\
\hline 15 & 10158 & 3927 & 0.241 \\
\hline 15 & 9090 & 3461 & 0.237 \\
\hline 20 & 12658 & 5678 & 0.279 \\
\hline 20 & 12978 & 5818 & 0.279 \\
\hline 20 & 11783 & 5290 & 0.279 \\
\hline 30 & 11488 & 6348 & 0.344 \\
\hline 30 & 11256 & 6346 & 0.351 \\
\hline 30 & 11061 & 5965 & 0.336 \\
\hline 40 & 11326 & 7301 & 0.401 \\
\hline 40 & 12349 & 7930 & 0.400 \\
\hline 40 & 10963 & 7068 & 0.401 \\
\hline 50 & 15176 & 10795 & 0.443 \\
\hline 50 & 14280 & 10205 & 0.445 \\
\hline 50 & 14189 & 10109 & 0.443 \\
\hline 60 & 11497 & 8888 & 0.481 \\
\hline 60 & 10590 & 8221 & 0.483 \\
\hline 60 & 10518 & 8128 & 0.481 \\
\hline 75 & 13935 & 11968 & 0.534 \\
\hline 75 & 13361 & 11534 & 0.537 \\
\hline 75 & 11761 & 10307 & 0.545 \\
\hline 90 & 10011 & 9254 & 0.575 \\
\hline 90 & 9741 & 9184 & 0.587 \\
\hline 90 & 8795 & 8076 & 0.571 \\
\hline 120 & 17462 & 17962 & 0.640 \\
\hline 120 & 15628 & 16108 & 0.641 \\
\hline
\end{tabular}




\section{Order in TBAF, (1.5 equiv).}

(E)-1

2-Iodothiophene

Tetra- $n$-butylammonium fluoride

Bis(dibenzylideneacetone)palladium $2.5 \mathrm{mM}$ (0.05 equiv)
$50 \mathrm{mM}$ (1.0 equiv)

$50 \mathrm{mM}$ (1.0 equiv)

$75 \mathrm{mM}$ (1.5 equiv)

Following the General Procedure, silanol $(E)-1$ (43 mg, $0.25 \mathrm{mmol})$ and biphenyl (11.8 $\mathrm{mg}$ ) were dissolved in TBAF solution $(375 \mu \mathrm{L}, 1.0 \mathrm{M}$ in THF, $0.375 \mathrm{mmol})$ in a flame dried 2neck round-bottomed flask, equipped with a stir bar and a septum under argon. Dry THF (3.60 $\mathrm{mL}$ ) was added and the clear, slightly-yellow solution was immersed into a water-bath at $23{ }^{\circ} \mathrm{C} \pm$ $1{ }^{\circ} \mathrm{C}$ for $30 \mathrm{~min}$. The 2-iodothiophene $(28 \mu \mathrm{L}, 0.25 \mathrm{mmol})$ was then added and the reaction was stirred for $1 \mathrm{~min}$. A suspension of $\operatorname{Pd}(\mathrm{dba})_{2}(7.2 \mathrm{mg}, 0.0125 \mathrm{mmol})$ in dry THF $(1.0 \mathrm{~mL})$ was added. The initially purple, turbid reaction mixture turns into a clear solution within 30 seconds. Aliquots $(\sim 200 \mu \mathrm{L}$ )were withdrawn after 1, 2, 3, 4, 5, 7, 10, 15, 20, 30, 40, 50, 60, 75, 90, and $120 \mathrm{~min}$. To quench, these samples were added to $100 \mu \mathrm{L}$ of a $20 \%$ aqueous solution of 2dimethylaminoethanethiol hydrochloride. The clear colorless to slightly-yellow solution was then filtered through a plug of silica gel, and the silica gel was washed with diethyl ether to give a total sample volume of $\sim 2 \mathrm{~mL}$. This sample was injected into the GC three times.

initial rate $=0.0140$

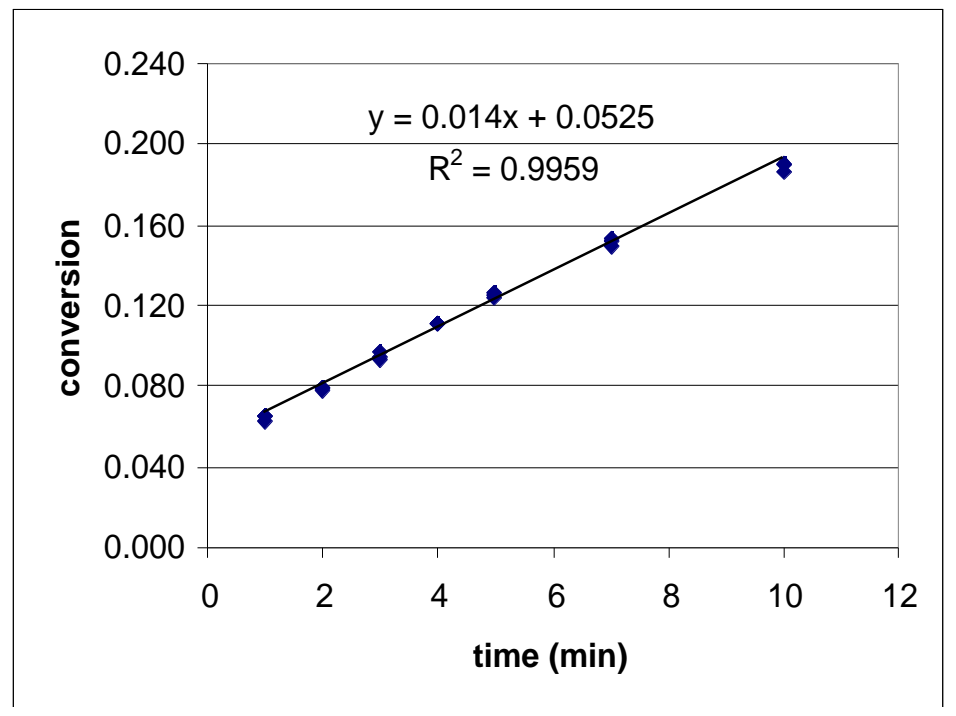




\begin{tabular}{|c|c|c|c|}
\hline time & area $\mathrm{A}$ & area 16 & conversion \\
\hline 1 & 7949 & 1165 & 0.065 \\
\hline 1 & 8412 & 1183 & 0.063 \\
\hline 1 & 7627 & 1108 & 0.065 \\
\hline 2 & 8986 & 1591 & 0.079 \\
\hline 2 & 9339 & 1652 & 0.079 \\
\hline 2 & 8118 & 1423 & 0.078 \\
\hline 3 & 9431 & 2044 & 0.096 \\
\hline 3 & 9571 & 2003 & 0.093 \\
\hline 3 & 9544 & 2016 & 0.094 \\
\hline 4 & 11865 & 2971 & 0.111 \\
\hline 4 & 12253 & 3065 & 0.111 \\
\hline 4 & 11312 & 2811 & 0.111 \\
\hline 5 & 10478 & 2983 & 0.127 \\
\hline 5 & 9394 & 2646 & 0.125 \\
\hline 5 & 9492 & 2642 & 0.124 \\
\hline 7 & 9667 & 3308 & 0.152 \\
\hline 7 & 9737 & 3350 & 0.153 \\
\hline 7 & 9626 & 3238 & 0.150 \\
\hline 10 & 9429 & 4030 & 0.190 \\
\hline 10 & 8173 & 3496 & 0.190 \\
\hline 10 & 9045 & 3799 & 0.187 \\
\hline 15 & 9581 & 5120 & 0.238 \\
\hline 15 & 8096 & 4489 & 0.247 \\
\hline 15 & 9463 & 5232 & 0.246 \\
\hline 20 & 11787 & 7551 & 0.285 \\
\hline 20 & 9337 & 6094 & 0.290 \\
\hline 20 & 9849 & 6383 & 0.288 \\
\hline 30 & 14143 & 11302 & 0.356 \\
\hline 30 & 11067 & 8844 & 0.356 \\
\hline 30 & 11302 & 8932 & 0.352 \\
\hline 40 & 11338 & 10355 & 0.406 \\
\hline 40 & 9628 & 8790 & 0.406 \\
\hline 40 & 9933 & 9262 & 0.415 \\
\hline 50 & 10903 & 11096 & 0.453 \\
\hline 50 & 8895 & 9133 & 0.457 \\
\hline 50 & 9152 & 9352 & 0.455 \\
\hline 60 & 9978 & 10928 & 0.487 \\
\hline 60 & 9195 & 10160 & 0.492 \\
\hline 60 & 8789 & 9846 & 0.499 \\
\hline 75 & 10816 & 13235 & 0.545 \\
\hline 75 & 10237 & 12681 & 0.551 \\
\hline 75 & 8847 & 10793 & 0.543 \\
\hline 90 & 14208 & 18626 & 0.583 \\
\hline 90 & 11814 & 15462 & 0.582 \\
\hline 90 & 12582 & 16485 & 0.583 \\
\hline 120 & 21059 & 31228 & 0.660 \\
\hline 120 & 11404 & 16854 & 0.658 \\
\hline
\end{tabular}




\section{Order in TBAF, (1.5 equiv).}

(E)-1

2-Iodothiophene

Tetra- $n$-butylammonium fluoride

Bis(dibenzylideneacetone)palladium $2.5 \mathrm{mM}$ (0.05 equiv)
$50 \mathrm{mM}$ (1.0 equiv)

$50 \mathrm{mM}$ (1.0 equiv)

$75 \mathrm{mM}$ (1.5 equiv)

Following the General Procedure, silanol $(E)-1$ (43 mg, $0.25 \mathrm{mmol})$ and biphenyl $(21.5$ $\mathrm{mg}$ ) were dissolved in TBAF solution $(375 \mu \mathrm{L}, 1.0 \mathrm{M}$ in THF, $0.375 \mathrm{mmol})$ in a flame dried 2neck round-bottomed flask, equipped with a stir bar and a septum under argon. Dry THF (3.60 $\mathrm{mL}$ ) was added and the clear, slightly-yellow solution was immersed into a water-bath at $23{ }^{\circ} \mathrm{C} \pm$ $1{ }^{\circ} \mathrm{C}$ for $30 \mathrm{~min}$. The 2-iodothiophene $(28 \mu \mathrm{L}, 0.25 \mathrm{mmol})$ was then added and the reaction was stirred for $1 \mathrm{~min}$. A suspension of $\operatorname{Pd}(\mathrm{dba})_{2}(7.2 \mathrm{mg}, 0.0125 \mathrm{mmol})$ in dry THF $(1.0 \mathrm{~mL})$ was added. The initially purple, turbid reaction mixture turns into a clear solution within 30 seconds. Aliquots $(\sim 200 \mu \mathrm{L}$ )were withdrawn after 1, 2, 3, 4, 5, 7, 10, 15, 20, 30, 40, 50, 60, 75, 90, and $120 \mathrm{~min}$. To quench, these samples were added to $100 \mu \mathrm{L}$ of a $20 \%$ aqueous solution of 2dimethylaminoethanethiol hydrochloride. The clear colorless to slightly-yellow solution was then filtered through a plug of silica gel, and the silica gel was washed with diethyl ether to give a total sample volume of $\sim 2 \mathrm{~mL}$. This sample was injected into the GC three times.

initial rate $=0.0149$

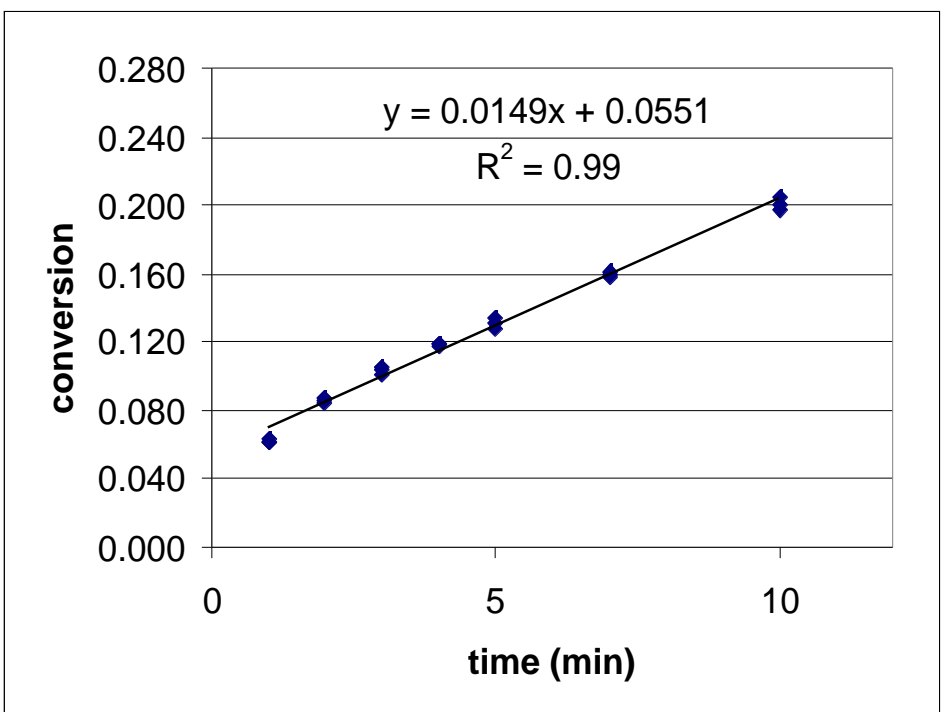




\begin{tabular}{|c|c|c|c|}
\hline time & area $\mathrm{A}$ & area 16 & conversion \\
\hline 1 & 33869 & 2581 & 0.062 \\
\hline 1 & 17574 & 1350 & 0.062 \\
\hline 1 & 20589 & 1617 & 0.064 \\
\hline 2 & 25065 & 2596 & 0.084 \\
\hline 2 & 15794 & 1703 & 0.087 \\
\hline 2 & 16031 & 1701 & 0.086 \\
\hline 3 & 18810 & 2339 & 0.101 \\
\hline 3 & 14126 & 1838 & 0.106 \\
\hline 3 & 15098 & 1938 & 0.104 \\
\hline 4 & 18140 & 2670 & 0.119 \\
\hline 4 & 17082 & 2519 & 0.120 \\
\hline 4 & 16182 & 2330 & 0.117 \\
\hline 5 & 16538 & 2616 & 0.128 \\
\hline 5 & 13422 & 2169 & 0.131 \\
\hline 5 & 14901 & 2473 & 0.135 \\
\hline 7 & 19557 & 3837 & 0.159 \\
\hline 7 & 19067 & 3701 & 0.157 \\
\hline 7 & 16538 & 3289 & 0.161 \\
\hline 10 & 17114 & 4149 & 0.197 \\
\hline 10 & 16774 & 4143 & 0.200 \\
\hline 10 & 15148 & 3835 & 0.205 \\
\hline 15 & 16421 & 5145 & 0.254 \\
\hline 15 & 16351 & 5150 & 0.255 \\
\hline 15 & 15103 & 4798 & 0.258 \\
\hline 20 & 22892 & 8435 & 0.299 \\
\hline 20 & 22993 & 8474 & 0.299 \\
\hline 20 & 20204 & 7573 & 0.304 \\
\hline 30 & 12486 & 5733 & 0.372 \\
\hline 30 & 13181 & 6155 & 0.379 \\
\hline 30 & 11079 & 5176 & 0.379 \\
\hline 40 & 20017 & 10660 & 0.432 \\
\hline 40 & 21597 & 11400 & 0.428 \\
\hline 40 & 17705 & 9416 & 0.431 \\
\hline 50 & 20897 & 12098 & 0.469 \\
\hline 50 & 25938 & 15071 & 0.471 \\
\hline 50 & 20445 & 11876 & 0.471 \\
\hline 60 & 16718 & 10453 & 0.507 \\
\hline 60 & 20335 & 12880 & 0.514 \\
\hline 60 & 13492 & 8585 & 0.516 \\
\hline 75 & 16885 & 11774 & 0.565 \\
\hline 75 & 17609 & 12181 & 0.561 \\
\hline 75 & 15960 & 11055 & 0.562 \\
\hline 90 & 14086 & 10606 & 0.611 \\
\hline 90 & 15045 & 11334 & 0.611 \\
\hline 90 & 13407 & 10110 & 0.611 \\
\hline 120 & 17652 & 14595 & 0.670 \\
\hline 120 & 19451 & 16036 & 0.668 \\
\hline
\end{tabular}




\section{Order in TBAF, (2.0 equiv).}

(E)-1

2-Iodothiophene

Tetra- $n$-butylammonium fluoride

Bis(dibenzylideneacetone)palladium $2.5 \mathrm{mM}$ (0.05 equiv)
$50 \mathrm{mM}$ (1.0 equiv)

$50 \mathrm{mM}$ (1.0 equiv)

$100 \mathrm{mM}$ (2.0 equiv)

Following the General Procedure, silanol $(E)-1$ (43 mg, $0.25 \mathrm{mmol})$ and biphenyl (16.8 $\mathrm{mg}$ ) were dissolved in TBAF solution $(500 \mu \mathrm{L}, 1.0 \mathrm{M}$ in THF, $0.50 \mathrm{mmol})$ in a flame dried 2neck round-bottomed flask, equipped with a stir bar and a septum under argon. Dry THF (3.50 $\mathrm{mL}$ ) was added and the clear, slightly-yellow solution was immersed into a water-bath at $23{ }^{\circ} \mathrm{C} \pm$ $1{ }^{\circ} \mathrm{C}$ for $30 \mathrm{~min}$. The 2-iodothiophene $(28 \mu \mathrm{L}, 0.25 \mathrm{mmol})$ was then added and the reaction was stirred for $1 \mathrm{~min}$. A suspension of $\operatorname{Pd}(\mathrm{dba})_{2}(7.2 \mathrm{mg}, 0.0125 \mathrm{mmol})$ in dry THF $(1.0 \mathrm{~mL})$ was added. The initially purple, turbid reaction mixture turns into a clear solution within 30 seconds. Aliquots $(\sim 200 \mu \mathrm{L}$ )were withdrawn after 1, 2, 3, 4, 5, 7, 10, 15, 20, 30, 40, 50, 60, 75, 90, and $120 \mathrm{~min}$. To quench, these samples were added to $100 \mu \mathrm{L}$ of a $20 \%$ aqueous solution of 2dimethylaminoethanethiol hydrochloride. The clear colorless to slightly-yellow solution was then filtered through a plug of silica gel, and the silica gel was washed with diethyl ether to give a total sample volume of $\sim 2 \mathrm{~mL}$. This sample was injected into the $\mathrm{GC}$ three times.

$\mathrm{k}_{2 \mathrm{obs}}=0.0179 \mathrm{~mol} / \mathrm{l} \min$

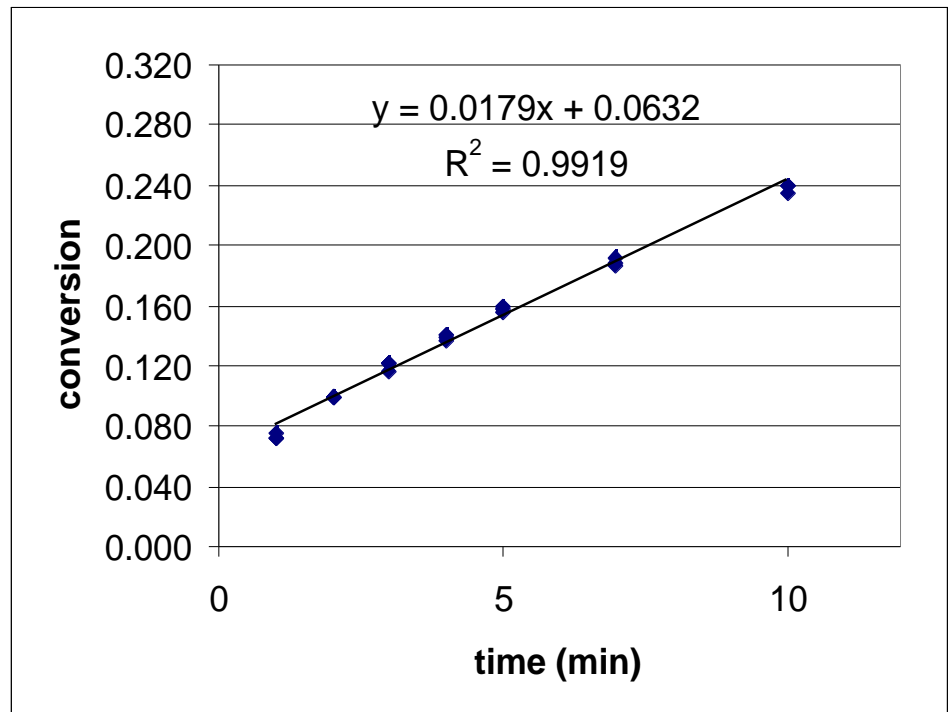




\begin{tabular}{|c|c|c|c|}
\hline time & area $\mathrm{A}$ & area 16 & conversion \\
\hline 1 & 10246 & 1209 & 0.075 \\
\hline 1 & 9548 & 1088 & 0.072 \\
\hline 1 & 10209 & 1159 & 0.072 \\
\hline 2 & 8888 & 1388 & 0.099 \\
\hline 2 & 9633 & 1510 & 0.099 \\
\hline 2 & 11386 & 1796 & 0.100 \\
\hline 3 & 10377 & 1982 & 0.121 \\
\hline 3 & 11078 & 2037 & 0.117 \\
\hline 3 & 13064 & 2504 & 0.121 \\
\hline 4 & 11563 & 2535 & 0.139 \\
\hline 4 & 11729 & 2590 & 0.140 \\
\hline 4 & 15343 & 3326 & 0.137 \\
\hline 5 & 9732 & 2420 & 0.158 \\
\hline 5 & 10996 & 2713 & 0.156 \\
\hline 5 & 13487 & 3390 & 0.159 \\
\hline 7 & 10070 & 3034 & 0.191 \\
\hline 7 & 14894 & 4391 & 0.187 \\
\hline 7 & 22742 & 6774 & 0.189 \\
\hline 10 & 12986 & 4893 & 0.239 \\
\hline 10 & 13033 & 4931 & 0.240 \\
\hline 10 & 16216 & 6007 & 0.235 \\
\hline 15 & 8042 & 3918 & 0.309 \\
\hline 15 & 10087 & 4898 & 0.308 \\
\hline 15 & 13060 & 6296 & 0.305 \\
\hline 20 & 8107 & 4697 & 0.367 \\
\hline 20 & 10928 & 6331 & 0.367 \\
\hline 20 & 13358 & 7709 & 0.366 \\
\hline 30 & 6766 & 5172 & 0.484 \\
\hline 30 & 9956 & 7542 & 0.480 \\
\hline 30 & 12607 & 9225 & 0.464 \\
\hline 40 & 9975 & 8743 & 0.555 \\
\hline 40 & 10426 & 9080 & 0.552 \\
\hline 40 & 13582 & 11992 & 0.559 \\
\hline 50 & 8666 & 8245 & 0.603 \\
\hline 50 & 9487 & 9318 & 0.622 \\
\hline 50 & 13735 & 13327 & 0.615 \\
\hline 60 & 11430 & 12191 & 0.676 \\
\hline 60 & 12608 & 13444 & 0.676 \\
\hline 60 & 17530 & 18867 & 0.682 \\
\hline 75 & 10392 & 12270 & 0.748 \\
\hline 75 & 11920 & 14018 & 0.745 \\
\hline 75 & 15470 & 18197 & 0.745 \\
\hline 90 & 9927 & 12753 & 0.814 \\
\hline 90 & 10956 & 13919 & 0.805 \\
\hline 90 & 13454 & 16735 & 0.788 \\
\hline 120 & 10464 & 14932 & 0.904 \\
\hline 120 & 12826 & 18145 & 0.896 \\
\hline
\end{tabular}




\section{Order in TBAF, (2.0 equiv).}

(E)-1

2-Iodothiophene

Tetra- $n$-butylammonium fluoride

Bis(dibenzylideneacetone)palladium $2.5 \mathrm{mM}$ (0.05 equiv)
$50 \mathrm{mM}$ (1.0 equiv)

$50 \mathrm{mM}$ (1.0 equiv)

$100 \mathrm{mM}$ (2.0 equiv)

Following the General Procedure, silanol (E)-1 (43 mg, $0.25 \mathrm{mmol})$ and biphenyl $(21.5$ $\mathrm{mg}$ ) were dissolved in TBAF solution $(500 \mu \mathrm{L}, 1.0 \mathrm{M}$ in THF, $0.50 \mathrm{mmol})$ in a flame dried 2neck round-bottomed flask, equipped with a stir bar and a septum under argon. Dry THF (3.50 $\mathrm{mL}$ ) was added and the clear, slightly-yellow solution was immersed into a water-bath at $23{ }^{\circ} \mathrm{C} \pm$ $1{ }^{\circ} \mathrm{C}$ for $30 \mathrm{~min}$. The 2-iodothiophene $(28 \mu \mathrm{L}, 0.25 \mathrm{mmol})$ was then added and the reaction was stirred for $1 \mathrm{~min}$. A suspension of $\operatorname{Pd}(\mathrm{dba})_{2}(7.2 \mathrm{mg}, 0.0125 \mathrm{mmol})$ in dry THF $(1.0 \mathrm{~mL})$ was added. The initially purple, turbid reaction mixture turns into a clear solution within 30 seconds. Aliquots $(\sim 200 \mu \mathrm{L}$ )were withdrawn after 1, 2, 3, 4, 5, 7, 10, 15, 20, 30, 40, 50, 60, 75, 90, and $120 \mathrm{~min}$. To quench, these samples were added to $100 \mu \mathrm{L}$ of a $20 \%$ aqueous solution of 2dimethylaminoethanethiol hydrochloride. The clear colorless to slightly-yellow solution was then filtered through a plug of silica gel, and the silica gel was washed with diethyl ether to give a total sample volume of $\sim 2 \mathrm{~mL}$. This sample was injected into the GC three times.

initial rate $=0.016$

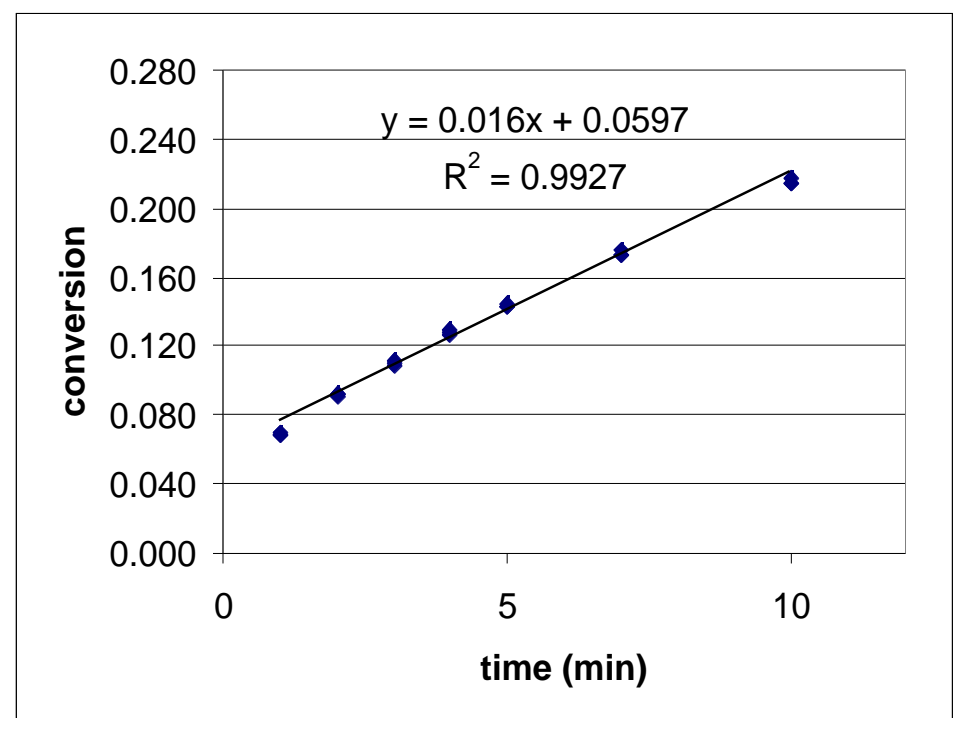




\begin{tabular}{|c|c|c|c|}
\hline time & area $\mathrm{A}$ & area 16 & conversion \\
\hline 1 & 13198 & 1134 & 0.070 \\
\hline 1 & 16369 & 1380 & 0.068 \\
\hline 1 & 20410 & 1724 & 0.068 \\
\hline 2 & 12335 & 1402 & 0.092 \\
\hline 2 & 15994 & 1791 & 0.091 \\
\hline 2 & 21921 & 2486 & 0.092 \\
\hline 3 & 14046 & 1935 & 0.112 \\
\hline 3 & 17118 & 2315 & 0.110 \\
\hline 3 & 20251 & 2718 & 0.109 \\
\hline 4 & 14877 & 2367 & 0.129 \\
\hline 4 & 16073 & 2551 & 0.129 \\
\hline 4 & 21170 & 3311 & 0.127 \\
\hline 5 & 12002 & 2107 & 0.142 \\
\hline 5 & 16700 & 2961 & 0.144 \\
\hline 5 & 21264 & 3745 & 0.143 \\
\hline 7 & 11902 & 2528 & 0.172 \\
\hline 7 & 13642 & 2946 & 0.175 \\
\hline 7 & 16735 & 3557 & 0.172 \\
\hline 10 & 12811 & 3441 & 0.218 \\
\hline 10 & 15508 & 4116 & 0.215 \\
\hline 10 & 18430 & 4865 & 0.214 \\
\hline 15 & 12485 & 4276 & 0.278 \\
\hline 15 & 16406 & 5607 & 0.277 \\
\hline 15 & 17663 & 6053 & 0.278 \\
\hline 20 & 12457 & 5183 & 0.337 \\
\hline 20 & 15150 & 6316 & 0.338 \\
\hline 20 & 18013 & 7523 & 0.339 \\
\hline 30 & 11524 & 6304 & 0.444 \\
\hline 30 & 16416 & 8780 & 0.434 \\
\hline 30 & 18055 & 9687 & 0.435 \\
\hline 40 & 14321 & 9222 & 0.522 \\
\hline 40 & 20257 & 12821 & 0.513 \\
\hline 40 & 20030 & 12658 & 0.512 \\
\hline 50 & 14831 & 10583 & 0.579 \\
\hline 50 & 20199 & 14347 & 0.576 \\
\hline 50 & 23994 & 17039 & 0.576 \\
\hline 60 & 10795 & 8611 & 0.647 \\
\hline 60 & 13936 & 10991 & 0.639 \\
\hline 60 & 16987 & 13244 & 0.632 \\
\hline 75 & 12417 & 10979 & 0.717 \\
\hline 75 & 19024 & 16514 & 0.704 \\
\hline 75 & 23455 & 20201 & 0.698 \\
\hline 90 & 12651 & 11945 & 0.766 \\
\hline 90 & 16570 & 15701 & 0.768 \\
\hline 90 & 22569 & 21053 & 0.756 \\
\hline 120 & 10872 & 11459 & 0.855 \\
\hline 120 & 18025 & 18835 & 0.847 \\
\hline
\end{tabular}




\section{Order in TBAF, (2.0 equiv).}

(E)-1

2-Iodothiophene

Tetra- $n$-butylammonium fluoride

Bis(dibenzylideneacetone)palladium $2.5 \mathrm{mM}$ (0.05 equiv)
$50 \mathrm{mM}$ (1.0 equiv)

$50 \mathrm{mM}$ (1.0 equiv)

$100 \mathrm{mM}$ (2.0 equiv)

Following the General Procedure, silanol (E)-1 (43 mg, $0.25 \mathrm{mmol})$ and biphenyl $(23.2$ $\mathrm{mg}$ ) were dissolved in TBAF solution $(500 \mu \mathrm{L}, 1.0 \mathrm{M}$ in THF, $0.50 \mathrm{mmol})$ in a flame dried 2neck round-bottomed flask, equipped with a stir bar and a septum under argon. Dry THF (3.50 $\mathrm{mL}$ ) was added and the clear, slightly-yellow solution was immersed into a water-bath at $23{ }^{\circ} \mathrm{C} \pm$ $1{ }^{\circ} \mathrm{C}$ for $30 \mathrm{~min}$. The 2-iodothiophene $(28 \mu \mathrm{L}, 0.25 \mathrm{mmol})$ was then added and the reaction was stirred for $1 \mathrm{~min}$. A suspension of $\operatorname{Pd}(\mathrm{dba})_{2}(7.2 \mathrm{mg}, 0.0125 \mathrm{mmol})$ in dry THF $(1.0 \mathrm{~mL})$ was added. The initially purple, turbid reaction mixture turns into a clear solution within 30 seconds. Aliquots $(\sim 200 \mu \mathrm{L}$ )were withdrawn after 1, 2, 3, 4, 5, 7, 10, 15, 20, 30, 40, 50, 60, 75, 90, and $120 \mathrm{~min}$. To quench, these samples were added to $100 \mu \mathrm{L}$ of a $20 \%$ aqueous solution of 2dimethylaminoethanethiol hydrochloride. The clear colorless to slightly-yellow solution was then filtered through a plug of silica gel, and the silica gel was washed with diethyl ether to give a total sample volume of $\sim 2 \mathrm{~mL}$. This sample was injected into the GC three times.

initial rate $=0.0166$

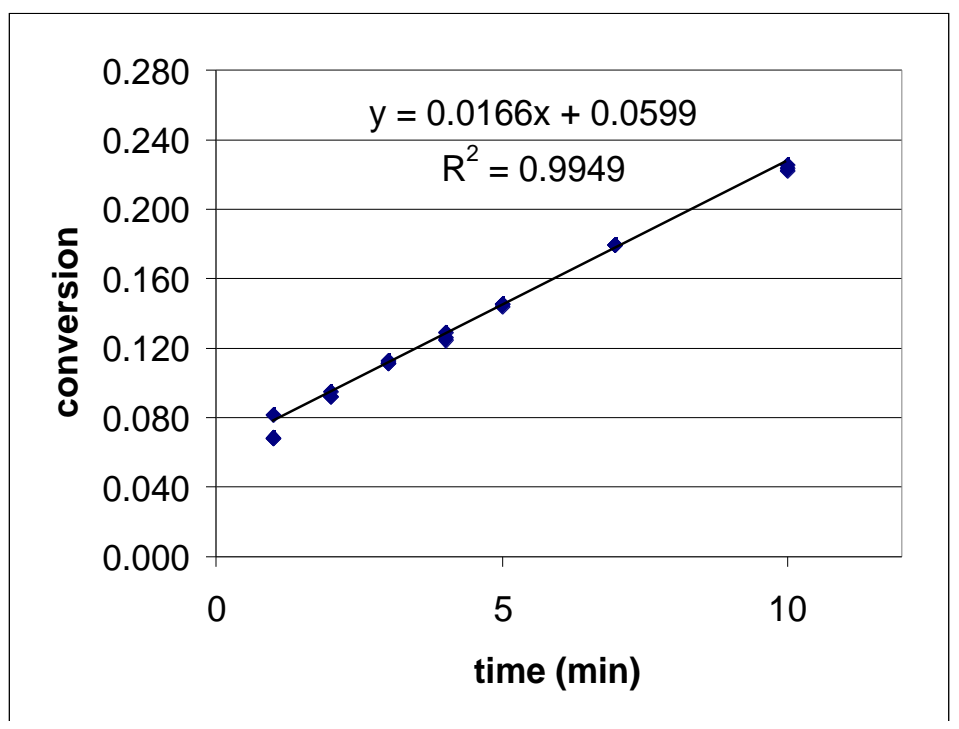




\begin{tabular}{|c|c|c|c|}
\hline time & area $\mathrm{A}$ & area 16 & conversion \\
\hline 1 & 13426 & 1057 & 0.069 \\
\hline 1 & 18783 & 1464 & 0.068 \\
\hline 1 & 21427 & 2005 & 0.082 \\
\hline 2 & 12970 & 1404 & 0.095 \\
\hline 2 & 19560 & 2067 & 0.092 \\
\hline 2 & 20202 & 2123 & 0.092 \\
\hline 3 & 12279 & 1568 & 0.112 \\
\hline 3 & 16679 & 2128 & 0.112 \\
\hline 3 & 17108 & 2210 & 0.113 \\
\hline 4 & 11461 & 1626 & 0.124 \\
\hline 4 & 14425 & 2120 & 0.129 \\
\hline 4 & 14031 & 2027 & 0.126 \\
\hline 5 & 17762 & 2931 & 0.144 \\
\hline 5 & 19537 & 3234 & 0.145 \\
\hline 5 & 19696 & 3274 & 0.145 \\
\hline 7 & 12446 & 2542 & 0.179 \\
\hline 7 & 15796 & 3227 & 0.179 \\
\hline 7 & 17514 & 3584 & 0.179 \\
\hline 10 & 11643 & 2968 & 0.223 \\
\hline 10 & 15098 & 3882 & 0.225 \\
\hline 10 & 17594 & 4463 & 0.222 \\
\hline 15 & 12240 & 4033 & 0.288 \\
\hline 15 & 15499 & 5105 & 0.288 \\
\hline 15 & 17104 & 5623 & 0.288 \\
\hline 20 & 14698 & 5740 & 0.342 \\
\hline 20 & 20518 & 8121 & 0.346 \\
\hline 20 & 26931 & 10787 & 0.350 \\
\hline 30 & 14418 & 7288 & 0.442 \\
\hline 30 & 18203 & 9217 & 0.443 \\
\hline 30 & 17828 & 9013 & 0.442 \\
\hline 40 & 14565 & 8763 & 0.526 \\
\hline 40 & 18152 & 11026 & 0.531 \\
\hline 40 & 19003 & 11456 & 0.527 \\
\hline 50 & 13145 & 8970 & 0.597 \\
\hline 50 & 17224 & 11612 & 0.590 \\
\hline 50 & 17834 & 12219 & 0.599 \\
\hline 60 & 13267 & 9762 & 0.644 \\
\hline 60 & 16756 & 12243 & 0.639 \\
\hline 60 & 16604 & 12293 & 0.648 \\
\hline 75 & 18408 & 15354 & 0.730 \\
\hline 75 & 20975 & 16986 & 0.709 \\
\hline 75 & 18099 & 14713 & 0.711 \\
\hline 90 & 15818 & 14026 & 0.776 \\
\hline 90 & 18116 & 16279 & 0.786 \\
\hline 90 & 17149 & 15171 & 0.774 \\
\hline 120 & 14207 & 13940 & 0.858 \\
\hline 120 & 17273 & 16942 & 0.858 \\
\hline
\end{tabular}




\section{Order in TBAF, (3.0 equiv).}

(E)-1

2-Iodothiophene

Tetra- $n$-butylammonium fluoride

Bis(dibenzylideneacetone)palladium $2.5 \mathrm{mM}$ (0.05 equiv)
$50 \mathrm{mM}$ (1.0 equiv)

$50 \mathrm{mM}$ (1.0 equiv)

$150 \mathrm{mM}$ (3.0 equiv)

Following the General Procedure, silanol (E)-1 (43 mg, $0.25 \mathrm{mmol})$ and biphenyl (24.0 $\mathrm{mg}$ ) were dissolved in TBAF solution $(750 \mu \mathrm{L}, 1.0 \mathrm{M}$ in THF, $0.75 \mathrm{mmol})$ in a flame dried 2neck round-bottomed flask, equipped with a stir bar and a septum under argon. Dry THF (3.25 $\mathrm{mL}$ ) was added and the clear, slightly-yellow solution was immersed into a water-bath at $23{ }^{\circ} \mathrm{C} \pm$ $1{ }^{\circ} \mathrm{C}$ for $30 \mathrm{~min}$. The 2-iodothiophene $(28 \mu \mathrm{L}, 0.25 \mathrm{mmol})$ was then added and the reaction was stirred for $1 \mathrm{~min}$. A suspension of $\operatorname{Pd}(\mathrm{dba})_{2}(7.2 \mathrm{mg}, 0.0125 \mathrm{mmol})$ in dry THF $(1.0 \mathrm{~mL})$ was added. The initially purple, turbid reaction mixture turns into a clear solution within 30 seconds. Aliquots $(\sim 200 \mu \mathrm{L}$ )were withdrawn after 1, 2, 3, 4, 5, 7, 10, 15, 20, 30, 40, 50, 60, 75, 90, and $120 \mathrm{~min}$. To quench, these samples were added to $100 \mu \mathrm{L}$ of a $20 \%$ aqueous solution of 2dimethylaminoethanethiol hydrochloride. The clear colorless to slightly-yellow solution was then filtered through a plug of silica gel, and the silica gel was washed with diethyl ether to give a total sample volume of $\sim 2 \mathrm{~mL}$. This sample was injected into the GC three times.

initial rate $=0.0104$

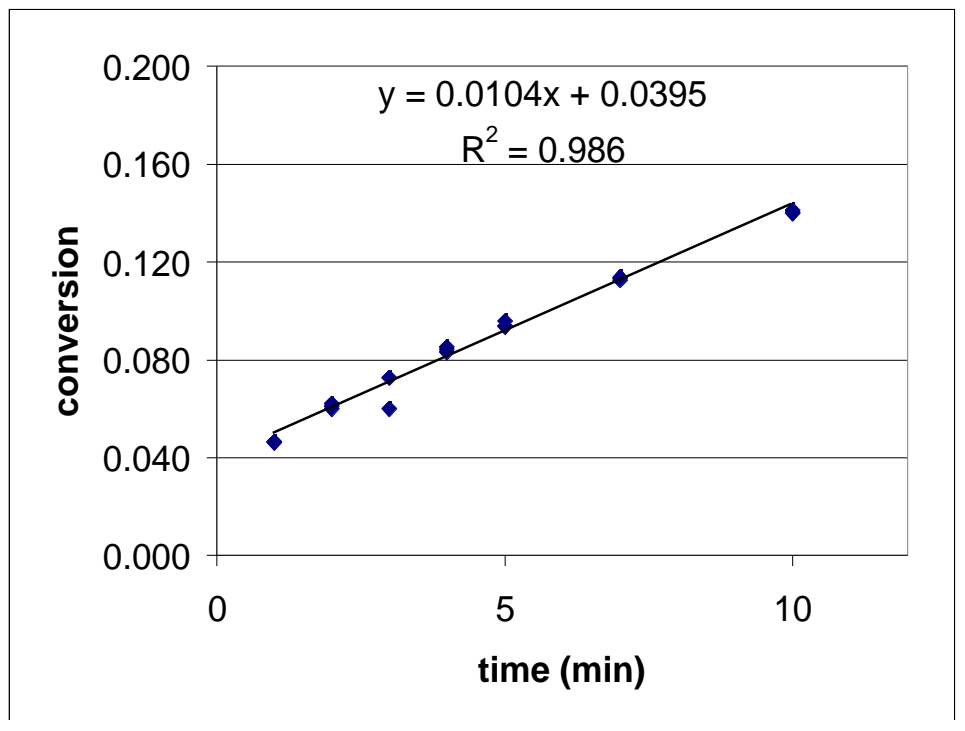




\begin{tabular}{|c|c|c|c|}
\hline time & area $\mathrm{A}$ & area 16 & conversion \\
\hline 1 & 34327 & 1762 & 0.046 \\
\hline 1 & 27260 & 1407 & 0.047 \\
\hline 1 & 29152 & 1490 & 0.046 \\
\hline 2 & 29480 & 2014 & 0.062 \\
\hline 2 & 21005 & 1400 & 0.060 \\
\hline 2 & 24431 & 1659 & 0.061 \\
\hline 3 & 27116 & 2177 & 0.073 \\
\hline 3 & 22895 & 1507 & 0.060 \\
\hline 3 & 25923 & 2073 & 0.072 \\
\hline 4 & 25068 & 2305 & 0.083 \\
\hline 4 & 22834 & 2154 & 0.085 \\
\hline 4 & 23322 & 2171 & 0.084 \\
\hline 5 & 35351 & 3663 & 0.094 \\
\hline 5 & 33908 & 3500 & 0.093 \\
\hline 5 & 36229 & 3818 & 0.095 \\
\hline 7 & 31385 & 3917 & 0.113 \\
\hline 7 & 27382 & 3432 & 0.113 \\
\hline 7 & 31529 & 3955 & 0.114 \\
\hline 10 & 29324 & 4529 & 0.140 \\
\hline 10 & 25988 & 4007 & 0.140 \\
\hline 10 & 29210 & 4555 & 0.141 \\
\hline 15 & 28271 & 5731 & 0.183 \\
\hline 15 & 23313 & 4652 & 0.181 \\
\hline 15 & 28267 & 5708 & 0.183 \\
\hline 20 & 30022 & 7312 & 0.220 \\
\hline 20 & 24731 & 6006 & 0.220 \\
\hline 20 & 29248 & 7176 & 0.222 \\
\hline 30 & 25189 & 8277 & 0.297 \\
\hline 30 & 21230 & 6950 & 0.296 \\
\hline 30 & 25648 & 8150 & 0.288 \\
\hline 40 & 30197 & 11954 & 0.358 \\
\hline 40 & 26925 & 10851 & 0.365 \\
\hline 40 & 34922 & 14030 & 0.364 \\
\hline 50 & 32775 & 15741 & 0.435 \\
\hline 50 & 26715 & 12761 & 0.432 \\
\hline 50 & 34509 & 16448 & 0.431 \\
\hline 60 & 30727 & 16585 & 0.489 \\
\hline 60 & 27092 & 14404 & 0.481 \\
\hline 60 & 30362 & 16115 & 0.480 \\
\hline 75 & 31712 & 19354 & 0.552 \\
\hline 75 & 27981 & 17177 & 0.556 \\
\hline 75 & 31807 & 19220 & 0.547 \\
\hline 90 & 24723 & 16723 & 0.612 \\
\hline 90 & 23599 & 15824 & 0.607 \\
\hline 90 & 29226 & 19551 & 0.605 \\
\hline 120 & 25737 & 19948 & 0.702 \\
\hline 120 & 22326 & 17142 & 0.695 \\
\hline
\end{tabular}




\section{Order in TBAF, (3.0 equiv).}

(E)-1

2-Iodothiophene

Tetra- $n$-butylammonium fluoride

Bis(dibenzylideneacetone)palladium $2.5 \mathrm{mM}$ (0.05 equiv)
$50 \mathrm{mM}$ (1.0 equiv)

$50 \mathrm{mM}$ (1.0 equiv)

$150 \mathrm{mM}$ (3.0 equiv)

Following the General Procedure, silanol (E)-1 (43 mg, $0.25 \mathrm{mmol})$ and biphenyl (20.0 $\mathrm{mg}$ ) were dissolved in TBAF solution $(750 \mu \mathrm{L}, 1.0 \mathrm{M}$ in THF, $0.75 \mathrm{mmol})$ in a flame dried 2neck round-bottomed flask, equipped with a stir bar and a septum under argon. Dry THF (3.25 $\mathrm{mL}$ ) was added and the clear, slightly-yellow solution was immersed into a water-bath at $23{ }^{\circ} \mathrm{C} \pm$ $1{ }^{\circ} \mathrm{C}$ for $30 \mathrm{~min}$. The 2-iodothiophene $(28 \mu \mathrm{L}, 0.25 \mathrm{mmol})$ was then added and the reaction was stirred for $1 \mathrm{~min}$. A suspension of $\operatorname{Pd}(\mathrm{dba})_{2}(7.2 \mathrm{mg}, 0.0125 \mathrm{mmol})$ in dry THF $(1.0 \mathrm{~mL})$ was added. The initially purple, turbid reaction mixture turns into a clear solution within 30 seconds. Aliquots $(\sim 200 \mu \mathrm{L}$ )were withdrawn after 1, 2, 3, 4, 5, 7, 10, 15, 20, 32, 42, 52, 62, 77, 92, and $122 \mathrm{~min}$. To quench, these samples were added to $100 \mu \mathrm{L}$ of a $20 \%$ aqueous solution of 2dimethylaminoethanethiol hydrochloride. The clear colorless to slightly-yellow solution was then filtered through a plug of silica gel, and the silica gel was washed with diethyl ether to give a total sample volume of $\sim 2 \mathrm{~mL}$. This sample was injected into the $\mathrm{GC}$ three times.

initial rate $=0.0120$

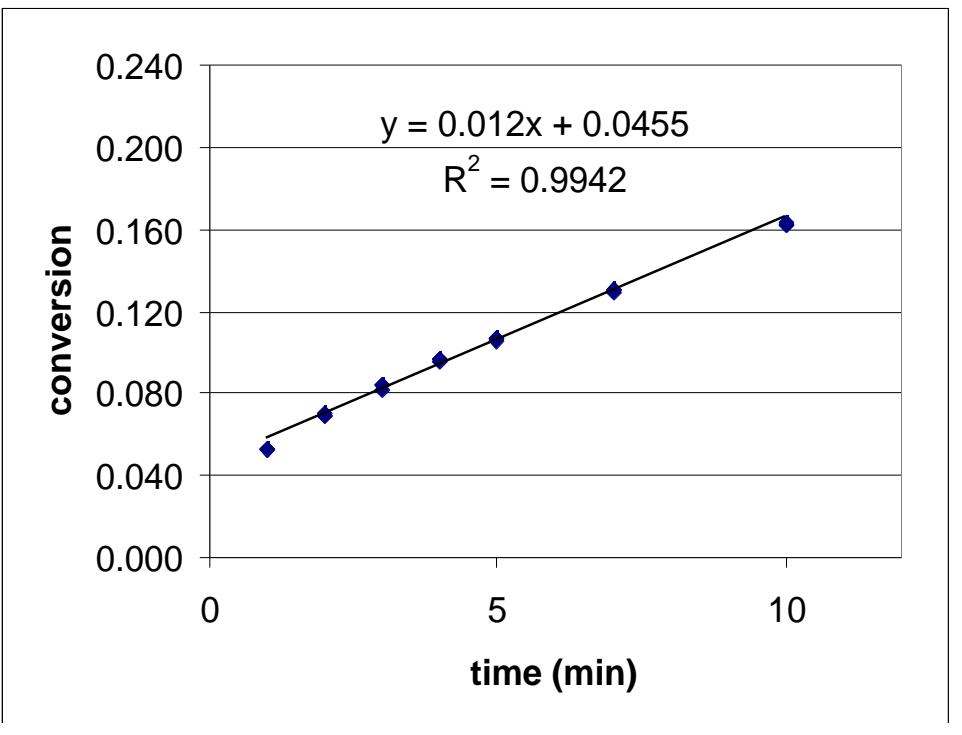




\begin{tabular}{|c|c|c|c|}
\hline time & $\operatorname{area} \mathrm{A}$ & area 16 & conversion \\
\hline 1 & 37536 & 2597 & 0.052 \\
\hline 1 & 32415 & 2282 & 0.053 \\
\hline 1 & 39935 & 2808 & 0.053 \\
\hline 2 & 20603 & 1891 & 0.069 \\
\hline 2 & 17494 & 1636 & 0.071 \\
\hline 2 & 20403 & 1864 & 0.069 \\
\hline 3 & 25750 & 2887 & 0.085 \\
\hline 3 & 20255 & 2208 & 0.082 \\
\hline 3 & 23538 & 2625 & 0.084 \\
\hline 4 & 31613 & 4006 & 0.096 \\
\hline 4 & 25730 & 3296 & 0.097 \\
\hline 4 & 27542 & 3551 & 0.097 \\
\hline 5 & 25713 & 3653 & 0.107 \\
\hline 5 & 21799 & 3101 & 0.107 \\
\hline 5 & 26608 & 3745 & 0.106 \\
\hline 7 & 28907 & 4994 & 0.130 \\
\hline 7 & 23218 & 3996 & 0.130 \\
\hline 7 & 27974 & 4853 & 0.131 \\
\hline 10 & 23829 & 5159 & 0.163 \\
\hline 10 & 26405 & 5681 & 0.162 \\
\hline 10 & 28981 & 6252 & 0.163 \\
\hline 15 & 29281 & 8351 & 0.215 \\
\hline 15 & 24312 & 6872 & 0.213 \\
\hline 15 & 31144 & 8842 & 0.214 \\
\hline 20 & 21650 & 7102 & 0.247 \\
\hline 20 & 19972 & 6556 & 0.248 \\
\hline 20 & 23194 & 7603 & 0.247 \\
\hline 30 & 32288 & 15020 & 0.351 \\
\hline 30 & 29285 & 13643 & 0.351 \\
\hline 30 & 34741 & 15998 & 0.347 \\
\hline 40 & 28567 & 16023 & 0.423 \\
\hline 40 & 29076 & 16274 & 0.422 \\
\hline 40 & 26350 & 14757 & 0.422 \\
\hline 50 & 23964 & 15635 & 0.492 \\
\hline 50 & 21366 & 13826 & 0.488 \\
\hline 50 & 21609 & 13971 & 0.488 \\
\hline 60 & 26944 & 19539 & 0.547 \\
\hline 60 & 27685 & 20268 & 0.552 \\
\hline 60 & 24884 & 18052 & 0.547 \\
\hline 75 & 31170 & 25440 & 0.616 \\
\hline 75 & 32168 & 26203 & 0.614 \\
\hline 75 & 35552 & 29108 & 0.618 \\
\hline 90 & 27110 & 24062 & 0.669 \\
\hline 90 & 27565 & 24214 & 0.663 \\
\hline 90 & 30125 & 26977 & 0.675 \\
\hline 120 & 27348 & 26976 & 0.744 \\
\hline 120 & 25472 & 25203 & 0.746 \\
\hline
\end{tabular}




\section{Order in TBAF, (3.0 equiv).}

(E)-1

2-Iodothiophene

Tetra- $n$-butylammonium fluoride

Bis(dibenzylideneacetone)palladium $2.5 \mathrm{mM}$ ( 0.05 equiv)
$50 \mathrm{mM}$ (1.0 equiv)

$50 \mathrm{mM}$ (1.0 equiv)

$150 \mathrm{mM}$ (3.0 equiv)

Following the General Procedure, silanol (E)-1 (43 mg, $0.25 \mathrm{mmol})$ and biphenyl (18.4 $\mathrm{mg}$ ) were dissolved in TBAF solution $(750 \mu \mathrm{L}, 1.0 \mathrm{M}$ in THF, $0.75 \mathrm{mmol})$ in a flame dried 2neck round-bottomed flask, equipped with a stir bar and a septum under argon. Dry THF (3.25 $\mathrm{mL}$ ) was added and the clear, slightly-yellow solution was immersed into a water-bath at $23{ }^{\circ} \mathrm{C} \pm$ $1{ }^{\circ} \mathrm{C}$ for $30 \mathrm{~min}$. The 2-iodothiophene $(28 \mu \mathrm{L}, 0.25 \mathrm{mmol})$ was then added and the reaction was stirred for $1 \mathrm{~min}$. A suspension of $\operatorname{Pd}(\mathrm{dba})_{2}(7.2 \mathrm{mg}, 0.0125 \mathrm{mmol})$ in dry THF $(1.0 \mathrm{~mL})$ was added. The initially purple, turbid reaction mixture turns into a clear solution within 30 seconds. Aliquots $(\sim 200 \mu \mathrm{L})$ were withdrawn after 1, 2, 3, 4, 5, 7, 10, 15, 20, 30, 40, 50, 60, 75, 90, and $120 \mathrm{~min}$. To quench, these samples were added to $100 \mu \mathrm{L}$ of a $20 \%$ aqueous solution of 2dimethylaminoethanethiol hydrochloride. The clear colorless to slightly-yellow solution was then filtered through a plug of silica gel, and the silica gel was washed with diethyl ether to give a total sample volume of $\sim 2 \mathrm{~mL}$. This sample was injected into the GC three times.

initial rate $=0.0133$

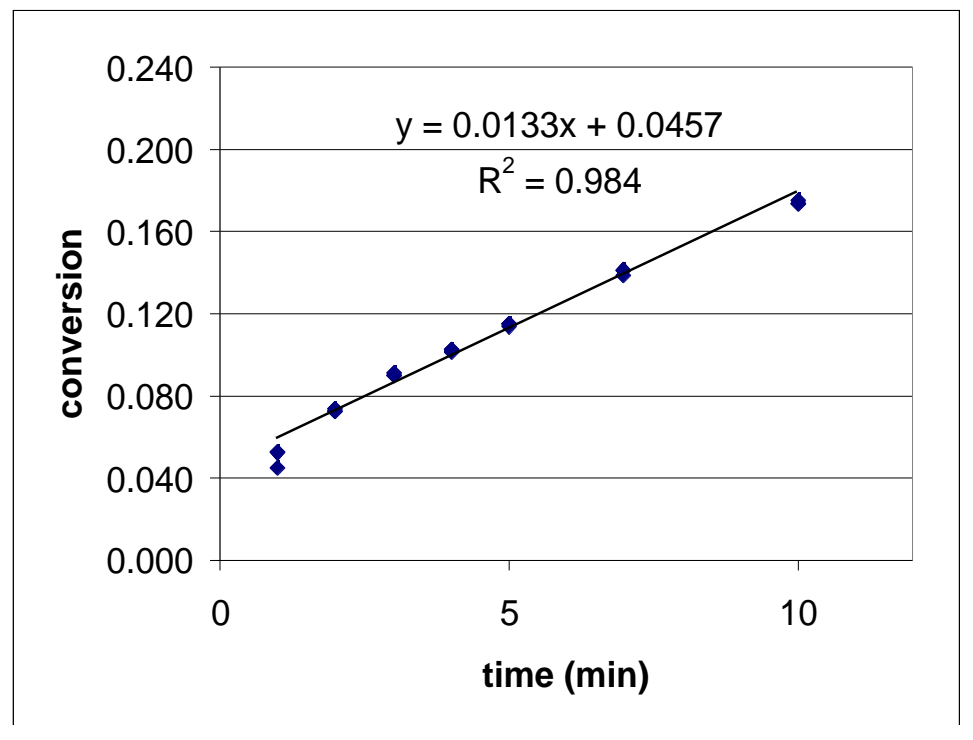




\begin{tabular}{|c|c|c|c|}
\hline time & $\operatorname{area} \mathrm{A}$ & area 16 & conversion \\
\hline 1 & 26483 & 1999 & 0.052 \\
\hline 1 & 26630 & 2036 & 0.053 \\
\hline 1 & 33436 & 2153 & 0.045 \\
\hline 2 & 26285 & 2786 & 0.074 \\
\hline 2 & 25467 & 2664 & 0.073 \\
\hline 2 & 32938 & 3449 & 0.073 \\
\hline 3 & 27358 & 3571 & 0.091 \\
\hline 3 & 24307 & 3191 & 0.091 \\
\hline 3 & 29113 & 3794 & 0.090 \\
\hline 4 & 25491 & 3758 & 0.102 \\
\hline 4 & 25990 & 3818 & 0.102 \\
\hline 4 & 31249 & 4587 & 0.102 \\
\hline 5 & 23610 & 3884 & 0.114 \\
\hline 5 & 22834 & 3801 & 0.116 \\
\hline 5 & 25604 & 4249 & 0.115 \\
\hline 7 & 26781 & 5434 & 0.141 \\
\hline 7 & 24651 & 4997 & 0.141 \\
\hline 7 & 32686 & 6560 & 0.139 \\
\hline 10 & 24573 & 6140 & 0.173 \\
\hline 10 & 23389 & 5856 & 0.174 \\
\hline 10 & 28791 & 7236 & 0.174 \\
\hline 15 & 25937 & 8467 & 0.227 \\
\hline 15 & 27135 & 8852 & 0.226 \\
\hline 15 & 34324 & 11275 & 0.228 \\
\hline 20 & 21371 & 8463 & 0.275 \\
\hline 20 & 24510 & 9710 & 0.275 \\
\hline 20 & 30401 & 12071 & 0.276 \\
\hline 30 & 17998 & 9444 & 0.364 \\
\hline 30 & 23897 & 12568 & 0.365 \\
\hline 30 & 32900 & 16782 & 0.354 \\
\hline 40 & 19772 & 12684 & 0.445 \\
\hline 40 & 24100 & 15324 & 0.441 \\
\hline 40 & 29184 & 18662 & 0.444 \\
\hline 50 & 22115 & 16347 & 0.513 \\
\hline 50 & 25586 & 18970 & 0.514 \\
\hline 50 & 30659 & 22374 & 0.506 \\
\hline 60 & 17409 & 14081 & 0.561 \\
\hline 60 & 21781 & 17746 & 0.565 \\
\hline 60 & 27019 & 21690 & 0.557 \\
\hline 75 & 21052 & 19090 & 0.629 \\
\hline 75 & 27579 & 24794 & 0.624 \\
\hline 75 & 31603 & 28490 & 0.626 \\
\hline 90 & 20998 & 20403 & 0.674 \\
\hline 90 & 28098 & 27310 & 0.674 \\
\hline 90 & 31044 & 30246 & 0.676 \\
\hline 120 & 27673 & 29581 & 0.742 \\
\hline 120 & 33622 & 36258 & 0.748 \\
\hline
\end{tabular}




\section{Order in TBAF, (4.0 equiv).}

(E)-1

2-Iodothiophene

Tetra- $n$-butylammonium fluoride $200 \mathrm{mM}$ (4.0 equiv)
$50 \mathrm{mM}$ (1.0 equiv)

$50 \mathrm{mM}$ (1.0 equiv)

Bis(dibenzylideneacetone)palladium $2.5 \mathrm{mM}$ (0.05 equiv)

Following the General Procedure, silanol (E)-1 (43 mg, $0.25 \mathrm{mmol})$ and biphenyl (15.4 $\mathrm{mg}$ ) were dissolved in TBAF solution $(1000 \mu \mathrm{L}, 1.0 \mathrm{M}$ in THF, $1.00 \mathrm{mmol})$ in a flame dried 2neck round-bottomed flask, equipped with a stir bar and a septum under argon. Dry THF (3.0 $\mathrm{mL}$ ) was added and the clear, slightly-yellow solution was immersed into a water-bath at $23{ }^{\circ} \mathrm{C} \pm$ $1{ }^{\circ} \mathrm{C}$ for $30 \mathrm{~min}$. The 2-iodothiophene $(28 \mu \mathrm{L}, 0.25 \mathrm{mmol})$ was then added and the reaction was stirred for $1 \mathrm{~min}$. A suspension of $\operatorname{Pd}(\mathrm{dba})_{2}(7.2 \mathrm{mg}, 0.0125 \mathrm{mmol})$ in dry THF $(1.0 \mathrm{~mL})$ was added. The initially purple, turbid reaction mixture turns into a clear solution within 30 seconds. Aliquots $(\sim 200 \mu \mathrm{L}$ )were withdrawn after 1, 2, 3, 4, 5, 7, 10, 15, 20, 30, 40, 50, 60, 75, 90, and $120 \mathrm{~min}$. To quench, these samples were added to $100 \mu \mathrm{L}$ of a $20 \%$ aqueous solution of 2dimethylaminoethanethiol hydrochloride. The clear colorless to slightly-yellow solution was then filtered through a plug of silica gel, and the silica gel was washed with diethyl ether to give a total sample volume of $\sim 2 \mathrm{~mL}$. This sample was injected into the GC three times.

initial rate $=0.009$

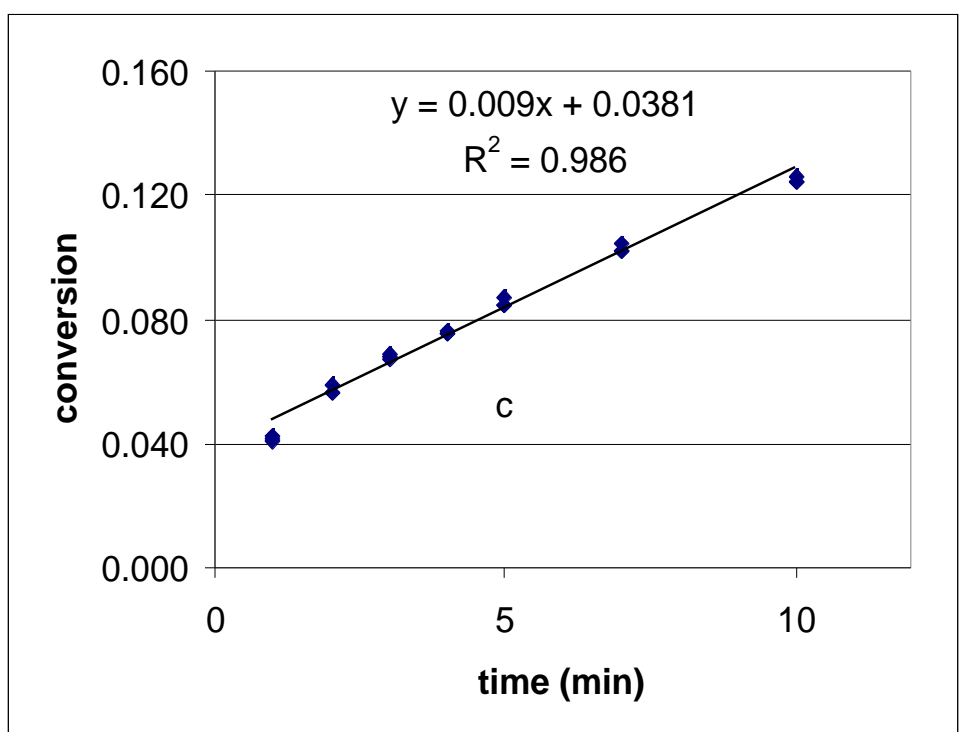




\begin{tabular}{|c|c|c|c|}
\hline time & area $\mathrm{A}$ & area 16 & conversion \\
\hline 1 & 12676 & 905 & 0.041 \\
\hline 1 & 18056 & 1263 & 0.041 \\
\hline 1 & 14833 & 1078 & 0.042 \\
\hline 2 & 12846 & 1245 & 0.056 \\
\hline 2 & 17327 & 1681 & 0.056 \\
\hline 2 & 13643 & 1375 & 0.059 \\
\hline 3 & 12672 & 1499 & 0.069 \\
\hline 3 & 13989 & 1642 & 0.068 \\
\hline 3 & 11133 & 1286 & 0.067 \\
\hline 4 & 14010 & 1836 & 0.076 \\
\hline 4 & 15998 & 2080 & 0.076 \\
\hline 4 & 14550 & 1881 & 0.075 \\
\hline 5 & 11591 & 1691 & 0.085 \\
\hline 5 & 12964 & 1947 & 0.087 \\
\hline 5 & 11899 & 1737 & 0.085 \\
\hline 7 & 14584 & 2567 & 0.102 \\
\hline 7 & 17108 & 3076 & 0.104 \\
\hline 7 & 15054 & 2635 & 0.102 \\
\hline 10 & 13293 & 2852 & 0.125 \\
\hline 10 & 16653 & 3622 & 0.126 \\
\hline 10 & 13758 & 2947 & 0.124 \\
\hline 15 & 11198 & 3125 & 0.162 \\
\hline 15 & 14266 & 3971 & 0.162 \\
\hline 15 & 12549 & 3467 & 0.160 \\
\hline 20 & 11240 & 3883 & 0.201 \\
\hline 20 & 14822 & 5047 & 0.198 \\
\hline 20 & 13142 & 4405 & 0.195 \\
\hline 30 & 15776 & 7172 & 0.264 \\
\hline 30 & 18563 & 8438 & 0.264 \\
\hline 30 & 17942 & 8087 & 0.262 \\
\hline 40 & 15848 & 9007 & 0.330 \\
\hline 40 & 18706 & 10343 & 0.321 \\
\hline 40 & 16468 & 9374 & 0.331 \\
\hline 50 & 17793 & 11949 & 0.390 \\
\hline 50 & 20085 & 13325 & 0.385 \\
\hline 50 & 18570 & 12366 & 0.387 \\
\hline 60 & 16662 & 12706 & 0.443 \\
\hline 60 & 17829 & 13812 & 0.450 \\
\hline 60 & 17617 & 13653 & 0.450 \\
\hline 75 & 16793 & 14591 & 0.505 \\
\hline 75 & 18123 & 15695 & 0.503 \\
\hline 75 & 17932 & 15637 & 0.506 \\
\hline 90 & 14334 & 14155 & 0.574 \\
\hline 90 & 15107 & 14975 & 0.576 \\
\hline 90 & 15783 & 15620 & 0.575 \\
\hline 120 & 15126 & 17385 & 0.668 \\
\hline 120 & 15067 & 17570 & 0.677 \\
\hline
\end{tabular}




\section{Order in TBAF, (4.0 equiv).}

(E)-1

2-Iodothiophene

Tetra- $n$-butylammonium fluoride $200 \mathrm{mM}$ (4.0 equiv)
$50 \mathrm{mM}$ (1.0 equiv)

$50 \mathrm{mM}$ (1.0 equiv)

Bis(dibenzylideneacetone)palladium $2.5 \mathrm{mM}$ (0.05 equiv)

Following the General Procedure, silanol (E)-1 (43 mg, $0.25 \mathrm{mmol})$ and biphenyl (15.1 $\mathrm{mg}$ ) were dissolved in TBAF solution $(1000 \mu \mathrm{L}, 1.0 \mathrm{M}$ in THF, $1.00 \mathrm{mmol})$ in a flame dried 2neck round-bottomed flask, equipped with a stir bar and a septum under argon. Dry THF (3.0 $\mathrm{mL}$ ) was added and the clear, slightly-yellow solution was immersed into a water-bath at $23{ }^{\circ} \mathrm{C} \pm$ $1{ }^{\circ} \mathrm{C}$ for $30 \mathrm{~min}$. The 2-iodothiophene $(28 \mu \mathrm{L}, 0.25 \mathrm{mmol})$ was then added and the reaction was stirred for $1 \mathrm{~min}$. A suspension of $\operatorname{Pd}(\mathrm{dba})_{2}(7.2 \mathrm{mg}, 0.0125 \mathrm{mmol})$ in dry THF $(1.0 \mathrm{~mL})$ was added. The initially purple, turbid reaction mixture turns into a clear solution within 30 seconds. Aliquots $(\sim 200 \mu \mathrm{L}$ )were withdrawn after 1, 2, 3, 4, 5, 7, 10, 15, 20, 30, 40, 50, 60, 75, 90, and $120 \mathrm{~min}$. To quench, these samples were added to $100 \mu \mathrm{L}$ of a $20 \%$ aqueous solution of 2dimethylaminoethanethiol hydrochloride. The clear colorless to slightly-yellow solution was then filtered through a plug of silica gel, and the silica gel was washed with diethyl ether to give a total sample volume of $\sim 2 \mathrm{~mL}$. This sample was injected into the $\mathrm{GC}$ three times.

initial rate $=0.0088$

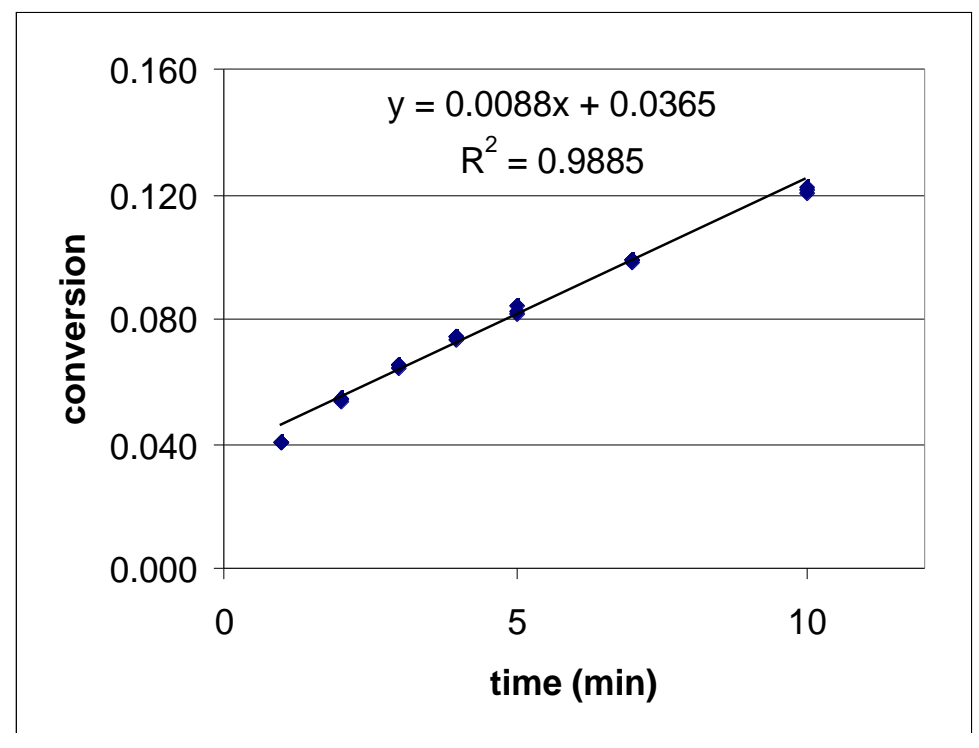




\begin{tabular}{|c|c|c|c|}
\hline time & area $\mathrm{A}$ & area 16 & conversion \\
\hline 1 & 13853 & 982 & 0.040 \\
\hline 1 & 14121 & 1006 & 0.041 \\
\hline 1 & 15089 & 1062 & 0.040 \\
\hline 2 & 13170 & 1244 & 0.054 \\
\hline 2 & 14848 & 1421 & 0.054 \\
\hline 2 & 14903 & 1419 & 0.054 \\
\hline 3 & 14508 & 1661 & 0.065 \\
\hline 3 & 18039 & 2064 & 0.065 \\
\hline 3 & 19641 & 2212 & 0.064 \\
\hline 4 & 14251 & 1845 & 0.074 \\
\hline 4 & 16265 & 2116 & 0.074 \\
\hline 4 & 16140 & 2105 & 0.074 \\
\hline 5 & 17318 & 2482 & 0.082 \\
\hline 5 & 20024 & 2957 & 0.084 \\
\hline 5 & 24006 & 3465 & 0.082 \\
\hline 7 & 16202 & 2785 & 0.098 \\
\hline 7 & 18280 & 3186 & 0.099 \\
\hline 7 & 19016 & 3299 & 0.099 \\
\hline 10 & 13537 & 2857 & 0.120 \\
\hline 10 & 15115 & 3219 & 0.121 \\
\hline 10 & 17615 & 3785 & 0.122 \\
\hline 15 & 14523 & 3894 & 0.153 \\
\hline 15 & 15196 & 4189 & 0.157 \\
\hline 15 & 16547 & 4515 & 0.155 \\
\hline 20 & 14580 & 4860 & 0.190 \\
\hline 20 & 15820 & 5227 & 0.188 \\
\hline 20 & 18896 & 6333 & 0.191 \\
\hline 30 & 12895 & 5724 & 0.253 \\
\hline 30 & 15967 & 6889 & 0.246 \\
\hline 30 & 16896 & 7479 & 0.252 \\
\hline 40 & 15402 & 8557 & 0.316 \\
\hline 40 & 18775 & 10255 & 0.311 \\
\hline 40 & 20208 & 11125 & 0.314 \\
\hline 50 & 13994 & 9059 & 0.369 \\
\hline 50 & 15436 & 10038 & 0.370 \\
\hline 50 & 15491 & 10073 & 0.370 \\
\hline 60 & 15409 & 11458 & 0.423 \\
\hline 60 & 17171 & 12697 & 0.421 \\
\hline 60 & 17862 & 13466 & 0.429 \\
\hline 75 & 14552 & 12516 & 0.490 \\
\hline 75 & 14405 & 12693 & 0.502 \\
\hline 75 & 15360 & 13353 & 0.495 \\
\hline 90 & 12230 & 11800 & 0.549 \\
\hline 90 & 13791 & 13272 & 0.548 \\
\hline 90 & 13357 & 13008 & 0.555 \\
\hline 120 & 14905 & 16846 & 0.644 \\
\hline 120 & 17118 & 19376 & 0.645 \\
\hline
\end{tabular}




\section{Order in TBAF, (4.0 equiv).}

(E)-1

2-Iodothiophene

Tetra- $n$-butylammonium fluoride $200 \mathrm{mM}$ (4.0 equiv)
$50 \mathrm{mM}$ (1.0 equiv)

$50 \mathrm{mM}$ (1.0 equiv)

Bis(dibenzylideneacetone)palladium $2.5 \mathrm{mM}$ (0.05 equiv)

Following the General Procedure, silanol (E)-1 (43 mg, $0.25 \mathrm{mmol})$ and biphenyl (13.3 $\mathrm{mg}$ ) were dissolved in TBAF solution $(1000 \mu \mathrm{L}, 1.0 \mathrm{M}$ in THF, $1.00 \mathrm{mmol})$ in a flame dried 2neck round-bottomed flask, equipped with a stir bar and a septum under argon. Dry THF (3.0 $\mathrm{mL}$ ) was added and the clear, slightly-yellow solution was immersed into a water-bath at $23{ }^{\circ} \mathrm{C} \pm$ $1{ }^{\circ} \mathrm{C}$ for $30 \mathrm{~min}$. The 2-iodothiophene $(28 \mu \mathrm{L}, 0.25 \mathrm{mmol})$ was then added and the reaction was stirred for $1 \mathrm{~min}$. A suspension of $\operatorname{Pd}(\mathrm{dba})_{2}(7.2 \mathrm{mg}, 0.0125 \mathrm{mmol})$ in dry THF $(1.0 \mathrm{~mL})$ was added. The initially purple, turbid reaction mixture turns into a clear solution within 30 seconds. Aliquots $(\sim 200 \mu \mathrm{L}$ )were withdrawn after 1, 2, 3, 4, 5, 7, 10, 15, 20, 30, 40, 50, 60, 75, 90, and $120 \mathrm{~min}$. To quench, these samples were added to $100 \mu \mathrm{L}$ of a $20 \%$ aqueous solution of 2dimethylaminoethanethiol hydrochloride. The clear colorless to slightly-yellow solution was then filtered through a plug of silica gel, and the silica gel was washed with diethyl ether to give a total sample volume of $\sim 2 \mathrm{~mL}$. This sample was injected into the $\mathrm{GC}$ three times.

initial rate $=0.0099$

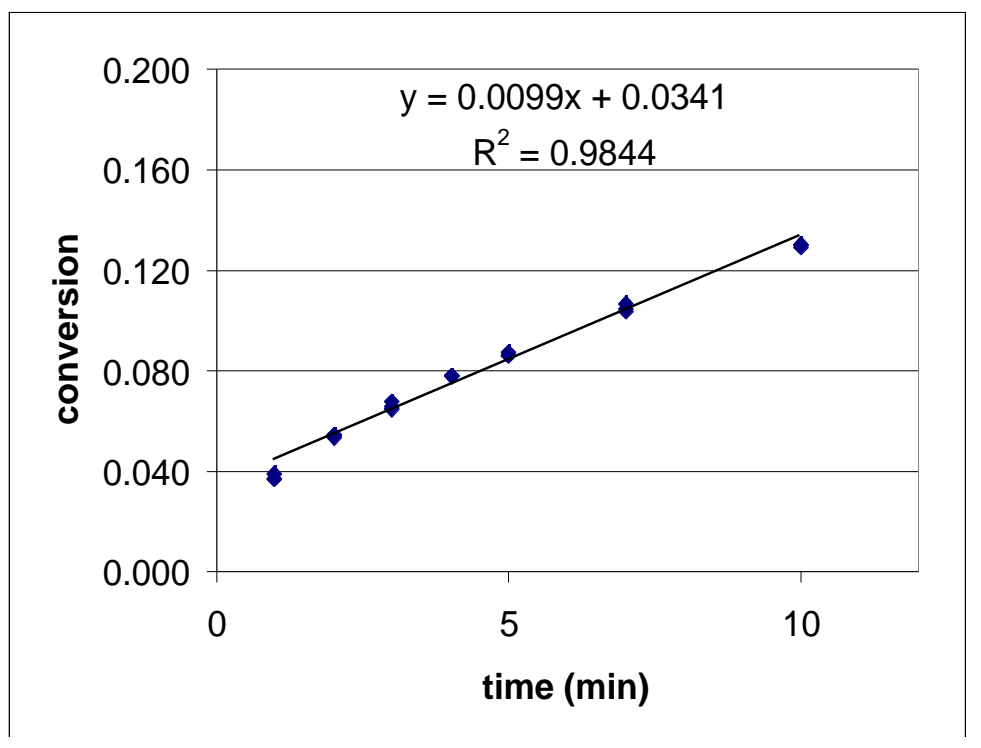




\begin{tabular}{|c|c|c|c|}
\hline time & area $\mathrm{A}$ & area 16 & conversion \\
\hline 1 & 12039 & 940 & 0.039 \\
\hline 1 & 14280 & 1061 & 0.037 \\
\hline 1 & 13827 & 1006 & 0.036 \\
\hline 2 & 14945 & 1617 & 0.054 \\
\hline 2 & 15748 & 1701 & 0.054 \\
\hline 2 & 16801 & 1794 & 0.054 \\
\hline 3 & 13967 & 1897 & 0.068 \\
\hline 3 & 14856 & 1953 & 0.066 \\
\hline 3 & 15196 & 1969 & 0.065 \\
\hline 4 & 15415 & 2407 & 0.078 \\
\hline 4 & 16075 & 2500 & 0.078 \\
\hline 4 & 15867 & 2462 & 0.078 \\
\hline 5 & 12047 & 2087 & 0.087 \\
\hline 5 & 12855 & 2196 & 0.086 \\
\hline 5 & 11048 & 1903 & 0.086 \\
\hline 7 & 12160 & 2504 & 0.103 \\
\hline 7 & 12701 & 2642 & 0.104 \\
\hline 7 & 10424 & 2213 & 0.106 \\
\hline 10 & 14710 & 3814 & 0.130 \\
\hline 10 & 15738 & 4088 & 0.130 \\
\hline 10 & 11216 & 2888 & 0.129 \\
\hline 15 & 13115 & 4339 & 0.166 \\
\hline 15 & 13773 & 4553 & 0.166 \\
\hline 15 & 12580 & 4180 & 0.167 \\
\hline 20 & 14611 & 5882 & 0.202 \\
\hline 20 & 13816 & 5543 & 0.201 \\
\hline 20 & 12115 & 4890 & 0.202 \\
\hline 30 & 11727 & 6100 & 0.261 \\
\hline 30 & 11303 & 6007 & 0.267 \\
\hline 30 & 10169 & 5365 & 0.265 \\
\hline 40 & 13341 & 8939 & 0.336 \\
\hline 40 & 14381 & 9613 & 0.335 \\
\hline 40 & 13722 & 9199 & 0.336 \\
\hline 50 & 13462 & 10577 & 0.394 \\
\hline 50 & 11956 & 9544 & 0.400 \\
\hline 50 & 11649 & 9147 & 0.394 \\
\hline 60 & 12051 & 10788 & 0.449 \\
\hline 60 & 11681 & 10508 & 0.451 \\
\hline 60 & 10703 & 9606 & 0.450 \\
\hline 75 & 12412 & 12784 & 0.517 \\
\hline 75 & 13659 & 14093 & 0.518 \\
\hline 75 & 10878 & 11133 & 0.513 \\
\hline 90 & 11849 & 13750 & 0.582 \\
\hline 90 & 11971 & 13703 & 0.574 \\
\hline 90 & 8013 & 9314 & 0.583 \\
\hline 120 & 14665 & 19712 & 0.674 \\
\hline 120 & 11135 & 14398 & 0.649 \\
\hline
\end{tabular}




\section{Order in TBAF, (5.0 equiv).}

(E)-1

2-Iodothiophene

Tetra- $n$-butylammonium fluoride

Bis(dibenzylideneacetone)palladium $2.5 \mathrm{mM}$ (0.05 equiv)
$50 \mathrm{mM}$ (1.0 equiv)

$50 \mathrm{mM}$ (1.0 equiv)

$250 \mathrm{mM}$ (5.0 equiv)

Following the General Procedure, silanol (E)-1 (43 mg, $0.25 \mathrm{mmol})$ and biphenyl (17.6 $\mathrm{mg}$ ) were dissolved in TBAF solution $(1250 \mu \mathrm{L}, 1.0 \mathrm{M}$ in THF, $1.25 \mathrm{mmol})$ in a flame dried 2neck round-bottomed flask, equipped with a stir bar and a septum under argon. Dry THF (2.75 $\mathrm{mL}$ ) was added and the clear, slightly-yellow solution was immersed into a water-bath at $23{ }^{\circ} \mathrm{C} \pm$ $1{ }^{\circ} \mathrm{C}$ for $30 \mathrm{~min}$. The 2-iodothiophene $(28 \mu \mathrm{L}, 0.25 \mathrm{mmol})$ was then added and the reaction was stirred for $1 \mathrm{~min}$. A suspension of $\operatorname{Pd}(\mathrm{dba})_{2}(7.2 \mathrm{mg}, 0.0125 \mathrm{mmol})$ in dry THF $(1.0 \mathrm{~mL})$ was added. The initially purple, turbid reaction mixture turns into a clear solution within 30 seconds. Aliquots $(\sim 200 \mu \mathrm{L}$ )were withdrawn after 1, 2, 3, 4, 5, 7, 10, 15, 20, 30, 40, 50, 60, 75, 90, and $120 \mathrm{~min}$. To quench, these samples were added to $100 \mu \mathrm{L}$ of a $20 \%$ aqueous solution of 2dimethylaminoethanethiol hydrochloride. The clear colorless to slightly-yellow solution was then filtered through a plug of silica gel, and the silica gel was washed with diethyl ether to give a total sample volume of $\sim 2 \mathrm{~mL}$. This sample was injected into the $\mathrm{GC}$ three times.

initial rate $=0.0073$

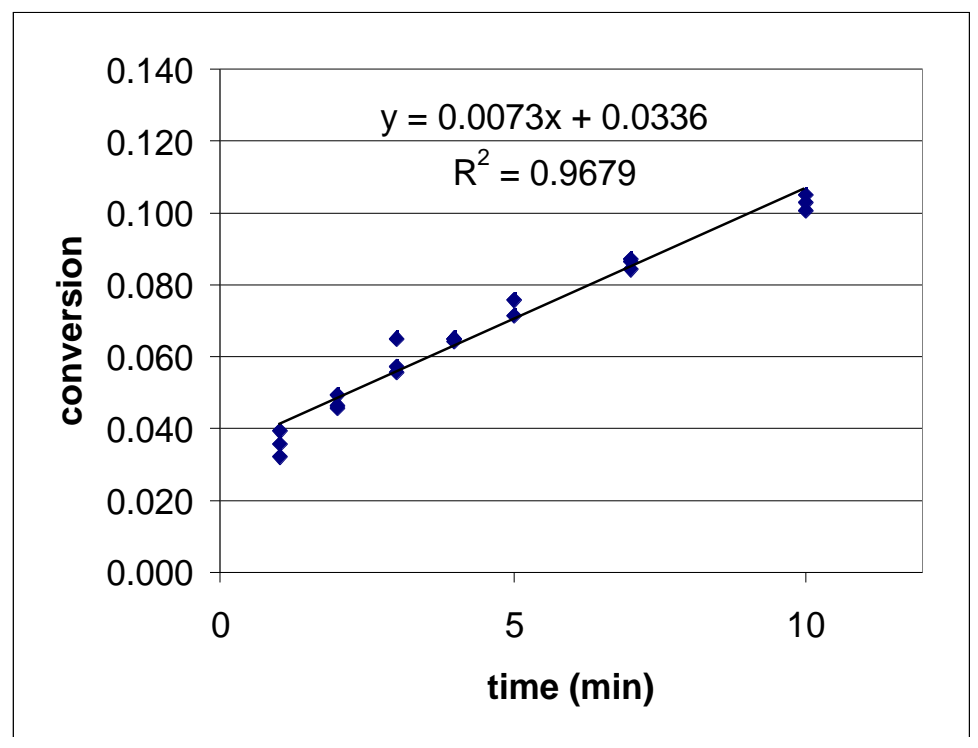




\begin{tabular}{|c|c|c|c|}
\hline time & area $\mathrm{A}$ & area 16 & conversion \\
\hline 1 & 8608 & 459 & 0.035 \\
\hline 1 & 8121 & 477 & 0.039 \\
\hline 1 & 9204 & 450 & 0.032 \\
\hline 2 & 9580 & 672 & 0.047 \\
\hline 2 & 7724 & 575 & 0.049 \\
\hline 2 & 8201 & 563 & 0.046 \\
\hline 3 & 8595 & 744 & 0.057 \\
\hline 3 & 7367 & 617 & 0.056 \\
\hline 3 & 7440 & 725 & 0.065 \\
\hline 4 & 8737 & 856 & 0.065 \\
\hline 4 & 8416 & 811 & 0.064 \\
\hline 4 & 8615 & 845 & 0.065 \\
\hline 5 & 9101 & 984 & 0.072 \\
\hline 5 & 8376 & 954 & 0.076 \\
\hline 7 & 8368 & 1095 & 0.087 \\
\hline 7 & 8397 & 1064 & 0.084 \\
\hline 7 & 13547 & 1758 & 0.086 \\
\hline 10 & 8281 & 1282 & 0.103 \\
\hline 10 & 8783 & 1394 & 0.105 \\
\hline 10 & 11639 & 1771 & 0.101 \\
\hline 15 & 8761 & 1776 & 0.135 \\
\hline 15 & 8461 & 1669 & 0.131 \\
\hline 15 & 8630 & 1705 & 0.131 \\
\hline 20 & 8649 & 2055 & 0.158 \\
\hline 20 & 8075 & 1926 & 0.158 \\
\hline 20 & 12056 & 2832 & 0.156 \\
\hline 30 & 7865 & 2397 & 0.202 \\
\hline 30 & 7002 & 2149 & 0.204 \\
\hline 30 & 8887 & 2805 & 0.209 \\
\hline 40 & 9221 & 3551 & 0.256 \\
\hline 40 & 9185 & 3552 & 0.257 \\
\hline 40 & 15151 & 5856 & 0.257 \\
\hline 50 & 6850 & 3165 & 0.307 \\
\hline 50 & 7387 & 3391 & 0.305 \\
\hline 50 & 11349 & 5332 & 0.312 \\
\hline 60 & 7631 & 4010 & 0.349 \\
\hline 60 & 7700 & 4075 & 0.351 \\
\hline 60 & 9304 & 4889 & 0.349 \\
\hline 75 & 6957 & 4414 & 0.421 \\
\hline 75 & 7156 & 4617 & 0.428 \\
\hline 75 & 8567 & 5426 & 0.420 \\
\hline 90 & 6896 & 4950 & 0.476 \\
\hline 90 & 8001 & 5789 & 0.480 \\
\hline 90 & 11356 & 8168 & 0.477 \\
\hline 120 & 6942 & 6028 & 0.576 \\
\hline 120 & 7627 & 6737 & 0.586 \\
\hline
\end{tabular}




\section{Order in TBAF, (5.0 equiv).}

(E)-1

2-Iodothiophene

Tetra- $n$-butylammonium fluoride

Bis(dibenzylideneacetone)palladium $2.5 \mathrm{mM}$ (0.05 equiv)
$50 \mathrm{mM}$ (1.0 equiv)

$50 \mathrm{mM}$ (1.0 equiv)

$250 \mathrm{mM}$ (5.0 equiv)

Following the General Procedure, silanol (E)-1 (43 mg, $0.25 \mathrm{mmol})$ and biphenyl (17.5 $\mathrm{mg}$ ) were dissolved in TBAF solution $(1250 \mu \mathrm{L}, 1.0 \mathrm{M}$ in THF, $1.25 \mathrm{mmol})$ in a flame dried 2neck round-bottomed flask, equipped with a stir bar and a septum under argon. Dry THF (2.75 $\mathrm{mL}$ ) was added and the clear, slightly-yellow solution was immersed into a water-bath at $23{ }^{\circ} \mathrm{C} \pm$ $1{ }^{\circ} \mathrm{C}$ for $30 \mathrm{~min}$. The 2-iodothiophene $(28 \mu \mathrm{L}, 0.25 \mathrm{mmol})$ was then added and the reaction was stirred for $1 \mathrm{~min}$. A suspension of $\operatorname{Pd}(\mathrm{dba})_{2}(7.2 \mathrm{mg}, 0.0125 \mathrm{mmol})$ in dry THF $(1.0 \mathrm{~mL})$ was added. The initially purple, turbid reaction mixture turns into a clear solution within 30 seconds. Aliquots $(\sim 200 \mu \mathrm{L}$ )were withdrawn after 1, 2, 3, 4, 5, 7, 10, 15, 20, 30, 40, 50, 60, 75, 90, and $120 \mathrm{~min}$. To quench, these samples were added to $100 \mu \mathrm{L}$ of a $20 \%$ aqueous solution of 2dimethylaminoethanethiol hydrochloride. The clear colorless to slightly-yellow solution was then filtered through a plug of silica gel, and the silica gel was washed with diethyl ether to give a total sample volume of $\sim 2 \mathrm{~mL}$. This sample was injected into the GC three times.

initial rate $=0.0061$

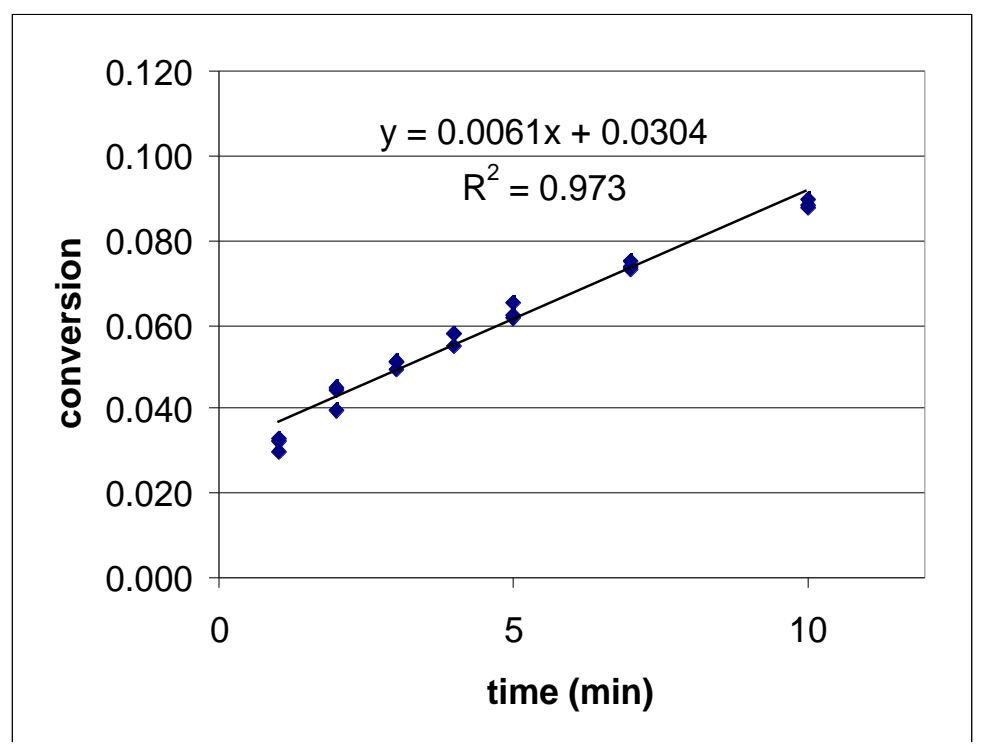




\begin{tabular}{|c|c|c|c|}
\hline time & area $\mathrm{A}$ & area 16 & conversion \\
\hline 1 & 8246 & 400 & 0.032 \\
\hline 1 & 8353 & 414 & 0.033 \\
\hline 1 & 12934 & 587 & 0.030 \\
\hline 2 & 10326 & 710 & 0.045 \\
\hline 2 & 10459 & 702 & 0.044 \\
\hline 2 & 13093 & 788 & 0.040 \\
\hline 3 & 9150 & 685 & 0.049 \\
\hline 3 & 8920 & 694 & 0.051 \\
\hline 3 & 14299 & 1108 & 0.051 \\
\hline 4 & 10454 & 915 & 0.058 \\
\hline 4 & 10075 & 836 & 0.055 \\
\hline 4 & 11764 & 1033 & 0.058 \\
\hline 5 & 9179 & 867 & 0.062 \\
\hline 5 & 9327 & 917 & 0.065 \\
\hline 5 & 9746 & 907 & 0.061 \\
\hline 7 & 9430 & 1050 & 0.073 \\
\hline 7 & 9489 & 1053 & 0.073 \\
\hline 7 & 10158 & 1149 & 0.075 \\
\hline 10 & 12341 & 1670 & 0.089 \\
\hline 10 & 12144 & 1609 & 0.087 \\
\hline 10 & 16246 & 2179 & 0.089 \\
\hline 15 & 11330 & 1893 & 0.110 \\
\hline 15 & 10744 & 1804 & 0.111 \\
\hline 15 & 13321 & 2276 & 0.113 \\
\hline 20 & 8997 & 1852 & 0.136 \\
\hline 20 & 8467 & 1625 & 0.127 \\
\hline 20 & 13930 & 2815 & 0.133 \\
\hline 30 & 8346 & 2294 & 0.181 \\
\hline 30 & 8729 & 2330 & 0.176 \\
\hline 30 & 13724 & 3698 & 0.178 \\
\hline 40 & 8226 & 2791 & 0.224 \\
\hline 40 & 8110 & 2732 & 0.222 \\
\hline 40 & 13439 & 4466 & 0.219 \\
\hline 50 & 7961 & 3136 & 0.260 \\
\hline 50 & 7590 & 3005 & 0.261 \\
\hline 50 & 11998 & 4726 & 0.260 \\
\hline 60 & 9112 & 4215 & 0.305 \\
\hline 60 & 9400 & 4327 & 0.304 \\
\hline 60 & 16303 & 7502 & 0.304 \\
\hline 75 & 8841 & 4837 & 0.361 \\
\hline 75 & 8985 & 5053 & 0.371 \\
\hline 75 & 14356 & 7955 & 0.366 \\
\hline 90 & 8082 & 5222 & 0.426 \\
\hline 90 & 8128 & 5269 & 0.428 \\
\hline 90 & 13065 & 8253 & 0.417 \\
\hline 120 & 10128 & 8059 & 0.525 \\
\hline 120 & 10098 & 7961 & 0.520 \\
\hline
\end{tabular}




\section{Order in TBAF, (5.0 equiv).}

(E)-1

2-Iodothiophene

Tetra- $n$-butylammonium fluoride

Bis(dibenzylideneacetone)palladium $2.5 \mathrm{mM}$ (0.05 equiv)
$50 \mathrm{mM}$ (1.0 equiv)

$50 \mathrm{mM}$ (1.0 equiv)

$250 \mathrm{mM}$ (5.0 equiv)

Following the General Procedure, silanol (E)-1 (43 mg, $0.25 \mathrm{mmol})$ and biphenyl (17.5 $\mathrm{mg}$ ) were dissolved in TBAF solution $(1250 \mu \mathrm{L}, 1.0 \mathrm{M}$ in THF, $1.25 \mathrm{mmol})$ in a flame dried 2neck round-bottomed flask, equipped with a stir bar and a septum under argon. Dry THF (2.75 $\mathrm{mL}$ ) was added and the clear, slightly-yellow solution was immersed into a water-bath at $23{ }^{\circ} \mathrm{C} \pm$ $1{ }^{\circ} \mathrm{C}$ for $30 \mathrm{~min}$. The 2-iodothiophene $(28 \mu \mathrm{L}, 0.25 \mathrm{mmol})$ was then added and the reaction was stirred for $1 \mathrm{~min}$. A suspension of $\operatorname{Pd}(\mathrm{dba})_{2}(7.2 \mathrm{mg}, 0.0125 \mathrm{mmol})$ in dry THF $(1.0 \mathrm{~mL})$ was added. The initially purple, turbid reaction mixture turns into a clear solution within 30 seconds. Aliquots $(\sim 200 \mu \mathrm{L})$ were withdrawn after 1, 2, 3, 4, 5, 7, 10, 15, 20, 30, 40, 50, 60, 75, 90, and $120 \mathrm{~min}$. To quench, these samples were added to $100 \mu \mathrm{L}$ of a $20 \%$ aqueous solution of 2dimethylaminoethanethiol hydrochloride. The clear colorless to slightly-yellow solution was then filtered through a plug of silica gel, and the silica gel was washed with diethyl ether to give a total sample volume of $\sim 2 \mathrm{~mL}$. This sample was injected into the GC three times.

initial rate $=0.0050$

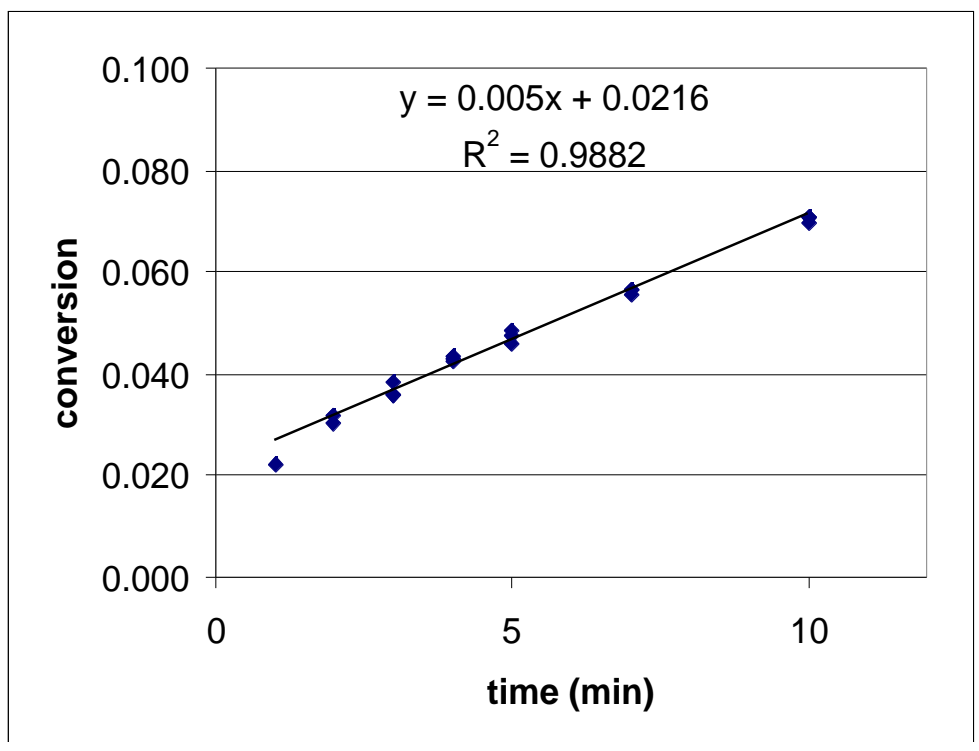




\begin{tabular}{|c|c|c|c|}
\hline time & area $\mathrm{A}$ & area 16 & conversion \\
\hline 1 & 13687 & 462 & 0.022 \\
\hline 2 & 8640 & 399 & 0.030 \\
\hline 2 & 15020 & 724 & 0.032 \\
\hline 3 & 7619 & 416 & 0.036 \\
\hline 3 & 8077 & 470 & 0.038 \\
\hline 3 & 13212 & 720 & 0.036 \\
\hline 4 & 8011 & 525 & 0.043 \\
\hline 4 & 8308 & 543 & 0.043 \\
\hline 4 & 10837 & 697 & 0.042 \\
\hline 5 & 7900 & 579 & 0.048 \\
\hline 5 & 9239 & 665 & 0.048 \\
\hline 5 & 15499 & 1079 & 0.046 \\
\hline 7 & 8505 & 729 & 0.057 \\
\hline 7 & 9063 & 780 & 0.057 \\
\hline 7 & 15912 & 1339 & 0.056 \\
\hline 10 & 9101 & 963 & 0.070 \\
\hline 10 & 9314 & 996 & 0.071 \\
\hline 10 & 16196 & 1729 & 0.070 \\
\hline 15 & 7617 & 1006 & 0.087 \\
\hline 15 & 8245 & 1161 & 0.093 \\
\hline 15 & 13904 & 1861 & 0.088 \\
\hline 20 & 7896 & 1299 & 0.109 \\
\hline 20 & 11615 & 1943 & 0.110 \\
\hline 20 & 13865 & 2237 & 0.106 \\
\hline 30 & 8265 & 1827 & 0.146 \\
\hline 30 & 9034 & 2010 & 0.147 \\
\hline 30 & 9493 & 2114 & 0.147 \\
\hline 40 & 10815 & 2971 & 0.181 \\
\hline 40 & 13645 & 3839 & 0.186 \\
\hline 40 & 24801 & 6662 & 0.177 \\
\hline 50 & 7652 & 2547 & 0.220 \\
\hline 50 & 8095 & 2692 & 0.219 \\
\hline 50 & 14294 & 4630 & 0.214 \\
\hline 60 & 8300 & 3188 & 0.253 \\
\hline 60 & 8573 & 3240 & 0.249 \\
\hline 60 & 12119 & 4641 & 0.253 \\
\hline 75 & 7624 & 3591 & 0.311 \\
\hline 75 & 8547 & 3949 & 0.305 \\
\hline 75 & 16297 & 7620 & 0.309 \\
\hline 90 & 7645 & 4051 & 0.350 \\
\hline 90 & 8699 & 4647 & 0.353 \\
\hline 90 & 18227 & 9649 & 0.349 \\
\hline 120 & 9316 & 6191 & 0.439 \\
\hline 120 & 10065 & 6719 & 0.441 \\
\hline
\end{tabular}




\section{Order in TBAF, (1.0 equiv).}

(E)-2

2-Iodothiophene

Tetra-n-butylammonium fluoride

Bis(dibenzylideneacetone)palladium $6.25 \mathrm{mM}$ (0.05 equiv)
$125 \mathrm{mM}$ (1.0 equiv)

$125 \mathrm{mM}$ (1.0 equiv)

$125 \mathrm{mM}$ (1.0 equiv)

Following the general procedure silanol $(E)-2(57.2 \mathrm{mg}, 0.25 \mathrm{mmol})$ and biphenyl $(9.2$ $\mathrm{mg}$ ) were dissolved in TBAF solution $(250 \mu \mathrm{L}, 1.0 \mathrm{M}$ in THF, $0.25 \mathrm{mmol})$ in a flame dried 2neck round-bottomed flask, equipped with a stir bar and a septum under argon. Dry THF (0.68 $\mathrm{mL}$ ) was added and the clear, slightly yellow solution was immersed into a water-bath at $23{ }^{\circ} \mathrm{C} \pm$ $1{ }^{\circ} \mathrm{C}$ for $30 \mathrm{~min}$. The 2-iodothiophene $(28 \mu \mathrm{L}, 0.25 \mathrm{mmol})$ was then added and the reaction was stirred for $1 \mathrm{~min}$. A suspension of $\operatorname{Pd}(\mathrm{dba})_{2}(7.2 \mathrm{mg}, 0.0125 \mathrm{mmol})$ in dry THF $(1.0 \mathrm{~mL})$ was added. The initially purple, turbid reaction mixture turns into a clear solution within 30 seconds. Aliquots ( $200 \mathrm{uL}$ were withdrawn after 1, 2, 3, 4, 5, 7, 10, 15, 20, 30, 40, 50, 60, 75, 90, and $120 \mathrm{~min}$. To quench, these samples were added to $100 \mathrm{uL}$ of an aqueous solution of 2dimethylaminoethanethiol hydrochloride $(20 \%)$. The clear colorless to slightly yellow solution was then filtered through a plug of silica-gel, and the silica-gel was washed with diethyl ether to give a total sample volume of $\sim 2 \mathrm{~mL}$. This sample was injected into the GC three times.

initial rate $=0.0173$

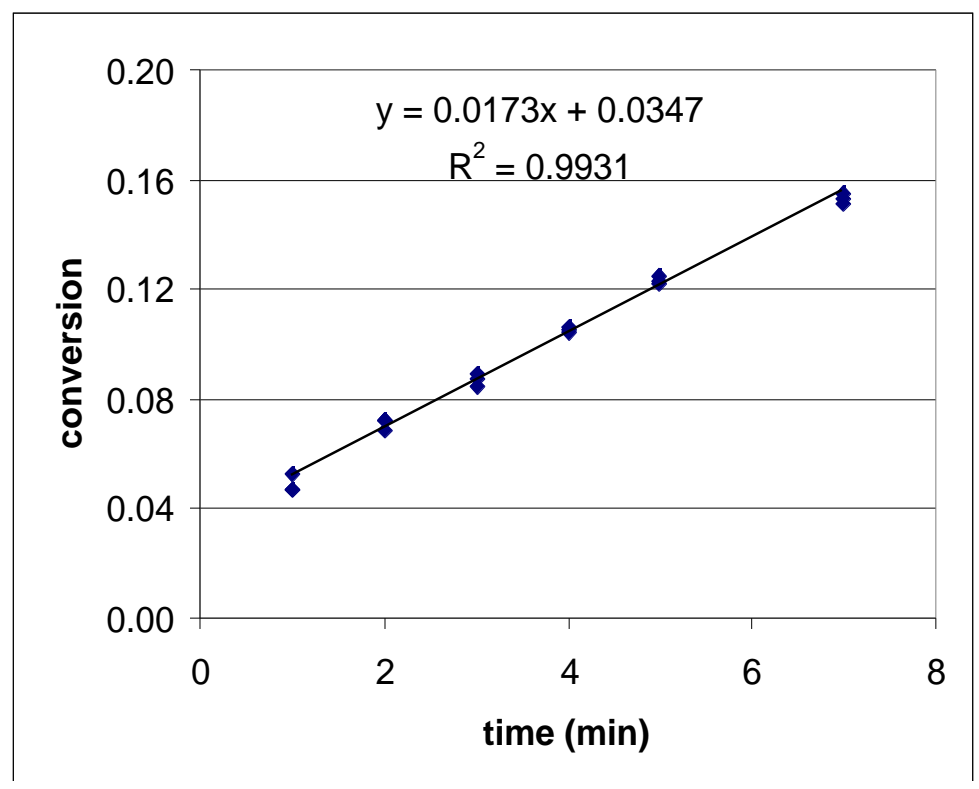




\begin{tabular}{|c|c|c|c|}
\hline time $(\min )$ & $\operatorname{area} \mathrm{A}$ & area 16 & conversion \\
\hline 1 & 9067 & 1378 & 0.053 \\
\hline 1 & 8313 & 1116 & 0.047 \\
\hline 1 & 9361 & 1266 & 0.047 \\
\hline 2 & 3664 & 724 & 0.069 \\
\hline 2 & 3785 & 784 & 0.072 \\
\hline 2 & 4405 & 922 & 0.073 \\
\hline 3 & 5545 & 1421 & 0.089 \\
\hline 3 & 6065 & 1530 & 0.088 \\
\hline 3 & 6031 & 1464 & 0.084 \\
\hline 4 & 3626 & 1098 & 0.105 \\
\hline 4 & 3686 & 1112 & 0.105 \\
\hline 4 & 3672 & 1122 & 0.106 \\
\hline 5 & 6236 & 2190 & 0.122 \\
\hline 5 & 6227 & 2246 & 0.125 \\
\hline 5 & 6497 & 2312 & 0.123 \\
\hline 7 & 7111 & 3129 & 0.153 \\
\hline 7 & 7000 & 3119 & 0.155 \\
\hline 7 & 6929 & 3020 & 0.151 \\
\hline 10 & 7715 & 4187 & 0.188 \\
\hline 10 & 6656 & 3735 & 0.195 \\
\hline 10 & 7567 & 4210 & 0.193 \\
\hline 15 & 6890 & 4821 & 0.243 \\
\hline 15 & 6343 & 4434 & 0.243 \\
\hline 15 & 6623 & 4671 & 0.245 \\
\hline 20 & 5522 & 4909 & 0.308 \\
\hline 20 & 5764 & 4994 & 0.301 \\
\hline 20 & 6095 & 5195 & 0.296 \\
\hline 30 & 5347 & 5785 & 0.375 \\
\hline 30 & 6185 & 6553 & 0.368 \\
\hline 30 & 5406 & 5958 & 0.382 \\
\hline 40 & 1561 & 1993 & 0.443 \\
\hline 40 & 1501 & 1820 & 0.421 \\
\hline 40 & 1506 & 1894 & 0.436 \\
\hline
\end{tabular}




\section{Order in TBAF, (1.0 equiv).}

(E)-2

2-Iodothiophene

Tetra-n-butylammonium fluoride

Bis(dibenzylideneacetone)palladium $6.25 \mathrm{mM}$ (0.05 equiv)
$125 \mathrm{mM}$ (1.0 equiv)

$125 \mathrm{mM}$ (1.0 equiv)

$125 \mathrm{mM}$ (1.0 equiv)

Following the general procedure silanol $(E)-2(57.2 \mathrm{mg}, 0.25 \mathrm{mmol})$ and biphenyl $(14.5$ $\mathrm{mg}$ ) were dissolved in TBAF solution $(250 \mu \mathrm{L}, 1.0 \mathrm{M}$ in THF, $0.25 \mathrm{mmol})$ in a flame dried 2neck round-bottomed flask, equipped with a stir bar and a septum under argon. Dry THF (0.68 $\mathrm{mL}$ ) was added and the clear, slightly yellow solution was immersed into a water-bath at $23{ }^{\circ} \mathrm{C} \pm$ $1{ }^{\circ} \mathrm{C}$ for $30 \mathrm{~min}$. The 2-iodothiophene $(28 \mu \mathrm{L}, 0.25 \mathrm{mmol})$ was then added and the reaction was stirred for $1 \mathrm{~min}$. A suspension of $\operatorname{Pd}(\mathrm{dba})_{2}(7.2 \mathrm{mg}, 0.0125 \mathrm{mmol})$ in dry THF $(1.0 \mathrm{~mL})$ was added. The initially purple, turbid reaction mixture turns into a clear solution within 30 seconds. Aliquots ( $200 \mathrm{uL}$ were withdrawn after 1, 2, 3, 4, 5, 7, 10, 15, 20, 30, 40, 50, 60, 75, 90, and $120 \mathrm{~min}$. To quench, these samples were added to $100 \mathrm{uL}$ of an aqueous solution of 2dimethylaminoethanethiol hydrochloride $(20 \%)$. The clear colorless to slightly yellow solution was then filtered through a plug of silica-gel, and the silica-gel was washed with diethyl ether to give a total sample volume of $\sim 2 \mathrm{~mL}$. This sample was injected into the GC three times.

initial rate $=0.0157$

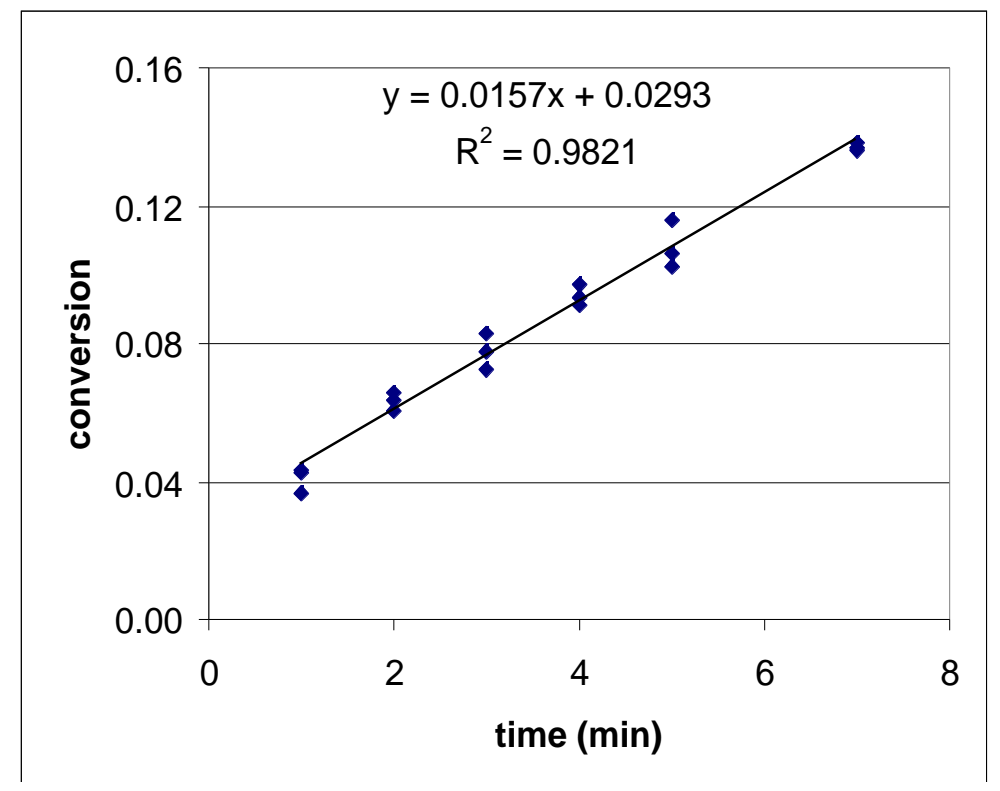




\begin{tabular}{|c|c|c|c|}
\hline time (min) & area $\mathrm{A}$ & area 16 & conversion \\
\hline 1 & 44881 & 3587 & 0.044 \\
\hline 1 & 16907 & 1142 & 0.037 \\
\hline 1 & 19605 & 1518 & 0.042 \\
\hline 2 & 22356 & 2589 & 0.063 \\
\hline 2 & 25042 & 2790 & 0.061 \\
\hline 2 & 20930 & 2522 & 0.066 \\
\hline 3 & 13325 & 2021 & 0.083 \\
\hline 3 & 13028 & 1846 & 0.077 \\
\hline 3 & 14452 & 1909 & 0.072 \\
\hline 4 & 13818 & 2308 & 0.091 \\
\hline 4 & 13624 & 2421 & 0.097 \\
\hline 4 & 16332 & 2801 & 0.094 \\
\hline 5 & 13247 & 2476 & 0.102 \\
\hline 5 & 12097 & 2571 & 0.116 \\
\hline 5 & 11730 & 2278 & 0.106 \\
\hline 7 & 12775 & 3202 & 0.137 \\
\hline 7 & 11800 & 2944 & 0.136 \\
\hline 7 & 9238 & 2342 & 0.139 \\
\hline 10 & 10613 & 3587 & 0.185 \\
\hline 10 & 9219 & 3382 & 0.201 \\
\hline 10 & 10190 & 3608 & 0.194 \\
\hline 15 & 8122 & 4035 & 0.272 \\
\hline 15 & 7566 & 3838 & 0.277 \\
\hline 15 & 8285 & 4304 & 0.284 \\
\hline 20 & 7270 & 4502 & 0.339 \\
\hline 20 & 7381 & 4547 & 0.337 \\
\hline 20 & 7120 & 4443 & 0.341 \\
\hline 30 & 8060 & 6628 & 0.450 \\
\hline 30 & 8601 & 6968 & 0.443 \\
\hline 30 & 8835 & 7116 & 0.440 \\
\hline 40 & 7139 & 7276 & 0.557 \\
\hline 40 & 7494 & 7528 & 0.549 \\
\hline 40 & 8425 & 8272 & 0.537 \\
\hline 50 & 11815 & 13148 & 0.609 \\
\hline 50 & 10994 & 12397 & 0.617 \\
\hline 50 & 10787 & 11956 & 0.606 \\
\hline 60 & 12166 & 14762 & 0.664 \\
\hline 60 & 9913 & 12020 & 0.663 \\
\hline 60 & 11223 & 13578 & 0.662 \\
\hline 75 & 12622 & 16515 & 0.715 \\
\hline 75 & 11206 & 14620 & 0.713 \\
\hline 75 & 12981 & 17090 & 0.720 \\
\hline 90 & 12982 & 18262 & 0.769 \\
\hline 90 & 12485 & 17550 & 0.769 \\
\hline 90 & 12431 & 17437 & 0.767 \\
\hline 120 & 12887 & 19033 & 0.808 \\
\hline 120 & 13298 & 19667 & 0.809 \\
\hline 120 & 13493 & 19847 & 0.804 \\
\hline
\end{tabular}




\section{Order in TBAF, (1.0 equiv).}

(E)-2

2-Iodothiophene

Tetra-n-butylammonium fluoride

Bis(dibenzylideneacetone)palladium $6.25 \mathrm{mM}$ (0.05 equiv)
$125 \mathrm{mM}$ (1.0 equiv)

$125 \mathrm{mM}$ (1.0 equiv)

$125 \mathrm{mM}$ (1.0 equiv)

Following the general procedure silanol $(E)-2(57.2 \mathrm{mg}, 0.25 \mathrm{mmol})$ and biphenyl $(10.2$ $\mathrm{mg}$ ) were dissolved in TBAF solution $(250 \mu \mathrm{L}, 1.0 \mathrm{M}$ in THF, $0.25 \mathrm{mmol})$ in a flame dried 2neck round-bottomed flask, equipped with a stir bar and a septum under argon. Dry THF (0.68 $\mathrm{mL}$ ) was added and the clear, slightly yellow solution was immersed into a water-bath at $23{ }^{\circ} \mathrm{C} \pm$ $1{ }^{\circ} \mathrm{C}$ for $30 \mathrm{~min}$. The 2-iodothiophene $(28 \mu \mathrm{L}, 0.25 \mathrm{mmol})$ was then added and the reaction was stirred for $1 \mathrm{~min}$. A suspension of $\operatorname{Pd}(\mathrm{dba})_{2}(7.2 \mathrm{mg}, 0.0125 \mathrm{mmol})$ in dry THF $(1.0 \mathrm{~mL})$ was added. The initially purple, turbid reaction mixture turns into a clear solution within 30 seconds. Aliquots ( $200 \mathrm{uL}$ were withdrawn after 1, 2, 3, 4, 5, 7, 10, 15, 20, 30, 40, 50, 60, 75, 90, and $120 \mathrm{~min}$. To quench, these samples were added to $100 \mathrm{uL}$ of an aqueous solution of 2dimethylaminoethanethiol hydrochloride $(20 \%)$. The clear colorless to slightly yellow solution was then filtered through a plug of silica-gel, and the silica-gel was washed with diethyl ether to give a total sample volume of $\sim 2 \mathrm{~mL}$. This sample was injected into the GC three times.

initial rate $=0.0184$

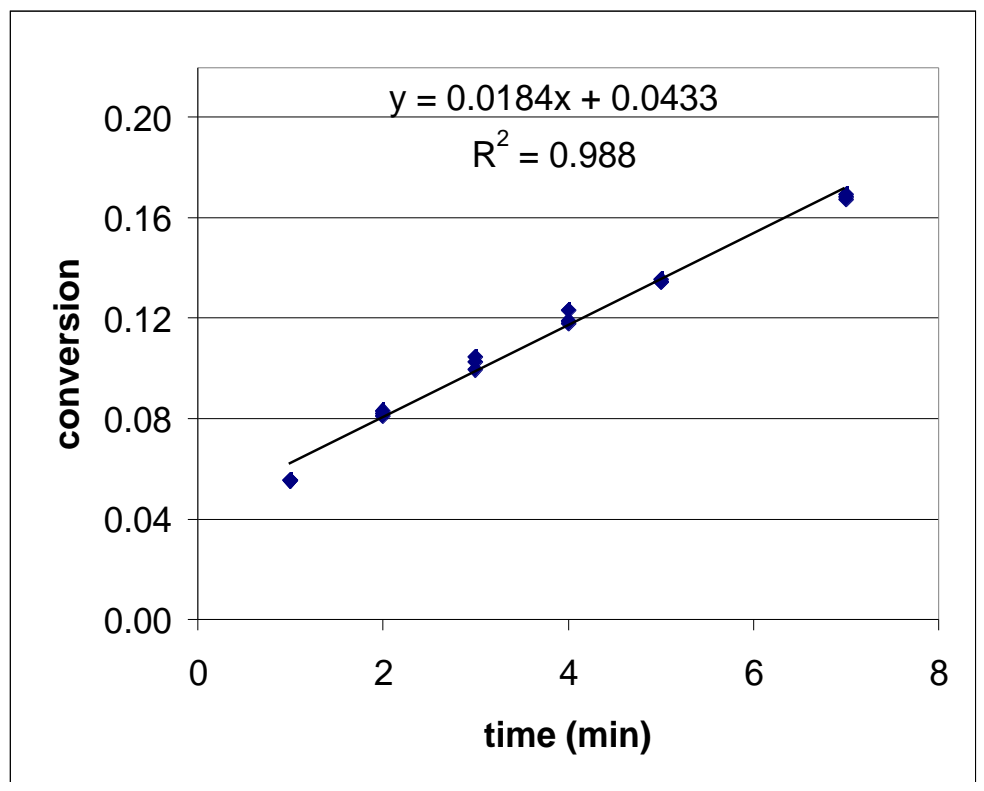




\begin{tabular}{|c|c|c|c|}
\hline time (min) & area $\mathrm{A}$ & area $\mathbf{1 6}$ & conversion \\
\hline 1 & 17697 & 2547 & 0.055 \\
\hline 1 & 16383 & 2378 & 0.056 \\
\hline 1 & 22856 & 3269 & 0.055 \\
\hline 2 & 11434 & 2411 & 0.081 \\
\hline 2 & 9266 & 2001 & 0.083 \\
\hline 2 & 9521 & 2039 & 0.082 \\
\hline 3 & 12323 & 3309 & 0.103 \\
\hline 3 & 25763 & 6710 & 0.100 \\
\hline 3 & 11106 & 3027 & 0.105 \\
\hline 4 & 33290 & 10190 & 0.118 \\
\hline 4 & 14450 & 4637 & 0.123 \\
\hline 4 & 33679 & 10420 & 0.119 \\
\hline 5 & 75014 & 26358 & 0.135 \\
\hline 5 & 36274 & 12788 & 0.136 \\
\hline 5 & 74017 & 25932 & 0.135 \\
\hline 7 & 23089 & 10177 & 0.170 \\
\hline 7 & 16444 & 7219 & 0.169 \\
\hline 7 & 23394 & 10183 & 0.167 \\
\hline 10 & 61908 & 33227 & 0.206 \\
\hline 10 & 31230 & 17002 & 0.209 \\
\hline 10 & 37095 & 20243 & 0.210 \\
\hline 15 & 143978 & 102113 & 0.273 \\
\hline 15 & 164387 & 117542 & 0.275 \\
\hline 15 & 45467 & 27013 & 0.229 \\
\hline 20 & 22365 & 16380 & 0.282 \\
\hline 20 & 20775 & 15234 & 0.282 \\
\hline 20 & 6980 & 5364 & 0.296 \\
\hline 30 & 15570 & 13758 & 0.340 \\
\hline 30 & 7125 & 6537 & 0.353 \\
\hline 30 & 15058 & 13142 & 0.336 \\
\hline 40 & 5596 & 6786 & 0.466 \\
\hline 40 & 10024 & 11626 & 0.446 \\
\hline 40 & 11560 & 13482 & 0.449 \\
\hline 50 & 5984 & 8616 & 0.554 \\
\hline 50 & 13054 & 17880 & 0.527 \\
\hline 50 & 9435 & 13029 & 0.531 \\
\hline 60 & 12915 & 19551 & 0.582 \\
\hline 60 & 15833 & 23914 & 0.581 \\
\hline 60 & 16188 & 24341 & 0.578 \\
\hline 75 & 19708 & 32066 & 0.626 \\
\hline 75 & 17436 & 28377 & 0.626 \\
\hline 75 & 21882 & 35508 & 0.624 \\
\hline 90 & 19086 & 33109 & 0.667 \\
\hline 90 & 22518 & 39132 & 0.668 \\
\hline 90 & 11949 & 20889 & 0.672 \\
\hline 120 & 16733 & 30856 & 0.709 \\
\hline
\end{tabular}


Order in TBAF, (2.0 equiv).

(E)-2

2-Iodothiophene

Tetra-n-butylammonium fluoride

Bis(dibenzylideneacetone)palladium $6.25 \mathrm{mM}$ (0.05 equiv)
$125 \mathrm{mM}$ (1.0 equiv)

$125 \mathrm{mM}$ (1.0 equiv)

$250 \mathrm{mM}$ (2.0 equiv)

Following the general procedure silanol $(E)-2(57.2 \mathrm{mg}, 0.25 \mathrm{mmol})$ and biphenyl $(13.8$ $\mathrm{mg}$ ) were dissolved in TBAF solution $(500 \mu \mathrm{L}, 1.0 \mathrm{M}$ in THF, $0.5 \mathrm{mmol})$ in a flame dried 2-neck round-bottomed flask, equipped with a stir bar and a septum under argon. Dry THF $(0.43 \mathrm{~mL})$ was added and the clear, slightly yellow solution was immersed into a water-bath at $23{ }^{\circ} \mathrm{C} \pm 1{ }^{\circ} \mathrm{C}$ for $30 \mathrm{~min}$. The 2-iodothiophene $(28 \mu \mathrm{L}, 0.25 \mathrm{mmol})$ was then added and the reaction was stirred for $1 \mathrm{~min}$. A suspension of $\operatorname{Pd}(\mathrm{dba})_{2}(7.2 \mathrm{mg}, 0.0125 \mathrm{mmol})$ in dry THF $(1.0 \mathrm{~mL})$ was added. The initially purple, turbid reaction mixture turns into a clear solution within 30 seconds. Aliquots ( $200 \mathrm{uL}$ were withdrawn after 1, 2, 3, 4, 5, 7, 10, 15, 20, 30, 40, 50, 60, 75, 90, and $120 \mathrm{~min}$. To quench, these samples were added to $100 \mathrm{uL}$ of an aqueous solution of 2dimethylaminoethanethiol hydrochloride $(20 \%)$. The clear colorless to slightly yellow solution was then filtered through a plug of silica-gel, and the silica-gel was washed with diethyl ether to give a total sample volume of $\sim 2 \mathrm{~mL}$. This sample was injected into the GC three times.

initial rate $=0.0132$

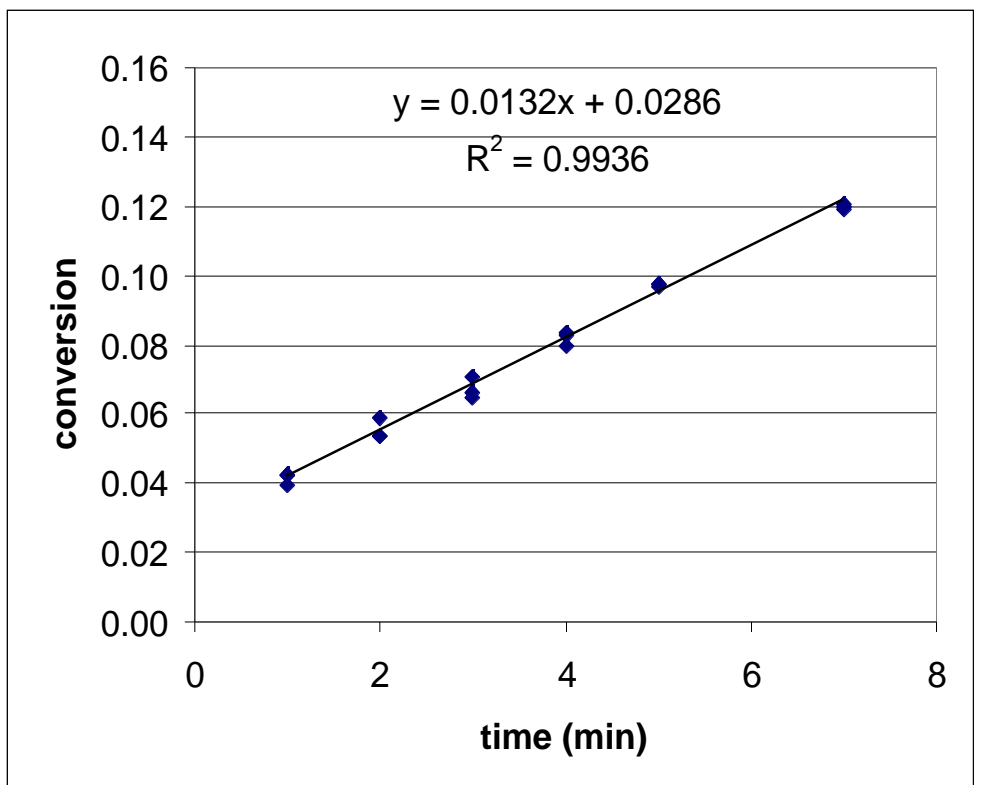




\begin{tabular}{|c|c|c|c|}
\hline time (min) & area $\mathrm{A}$ & area 16 & conversion \\
\hline 1 & 11312 & 855 & 0.042 \\
\hline 1 & 8892 & 722 & 0.042 \\
\hline 1 & 9005 & 687 & 0.040 \\
\hline 2 & 9061 & 927 & 0.053 \\
\hline 2 & 8528 & 968 & 0.059 \\
\hline 2 & 8866 & 912 & 0.054 \\
\hline 3 & 10271 & 1390 & 0.070 \\
\hline 3 & 10595 & 1319 & 0.065 \\
\hline 3 & 10979 & 1403 & 0.067 \\
\hline 4 & 10740 & 1720 & 0.083 \\
\hline 4 & 11536 & 1770 & 0.080 \\
\hline 4 & 10837 & 1727 & 0.083 \\
\hline 5 & 10574 & 1978 & 0.097 \\
\hline 5 & 10474 & 1941 & 0.096 \\
\hline 5 & 10832 & 2031 & 0.098 \\
\hline 7 & 13973 & 3216 & 0.120 \\
\hline 7 & 12833 & 2937 & 0.119 \\
\hline 7 & 14350 & 3329 & 0.121 \\
\hline 10 & 11297 & 3092 & 0.142 \\
\hline 10 & 11767 & 3140 & 0.139 \\
\hline 10 & 12424 & 3383 & 0.142 \\
\hline 15 & 12828 & 4254 & 0.173 \\
\hline 15 & 12133 & 4353 & 0.187 \\
\hline 15 & 14300 & 4986 & 0.181 \\
\hline 20 & 5734 & 2299 & 0.209 \\
\hline 20 & 5343 & 2324 & 0.226 \\
\hline 20 & 5261 & 2156 & 0.213 \\
\hline 30 & 15032 & 8709 & 0.302 \\
\hline 30 & 15673 & 8995 & 0.299 \\
\hline 30 & 15434 & 8908 & 0.300 \\
\hline 40 & 12109 & 8614 & 0.370 \\
\hline 40 & 11216 & 8416 & 0.391 \\
\hline 40 & 11931 & 8356 & 0.364 \\
\hline 50 & 11651 & 9448 & 0.422 \\
\hline 50 & 11842 & 9856 & 0.433 \\
\hline 50 & 11663 & 9434 & 0.421 \\
\hline 60 & 8241 & 7643 & 0.483 \\
\hline 60 & 8618 & 7910 & 0.478 \\
\hline 60 & 8658 & 7957 & 0.478 \\
\hline 75 & 10030 & 13265 & 0.688 \\
\hline 75 & 10619 & 14286 & 0.700 \\
\hline 75 & 10330 & 13639 & 0.687 \\
\hline 90 & 5949 & 6314 & 0.552 \\
\hline 90 & 5420 & 6155 & 0.591 \\
\hline 90 & 5649 & 6327 & 0.583 \\
\hline
\end{tabular}


Order in TBAF, (2.0 equiv).

(E)-2

2-Iodothiophene

Tetra-n-butylammonium fluoride

Bis(dibenzylideneacetone)palladium $6.25 \mathrm{mM}$ (0.05 equiv)
$125 \mathrm{mM}$ (1.0 equiv)

$125 \mathrm{mM}$ (1.0 equiv)

$250 \mathrm{mM}$ (2.0 equiv)

Following the general procedure silanol $(E)-2(57.2 \mathrm{mg}, 0.25 \mathrm{mmol})$ and biphenyl (13.7 $\mathrm{mg}$ ) were dissolved in TBAF solution $(500 \mu \mathrm{L}, 1.0 \mathrm{M}$ in THF, $0.5 \mathrm{mmol})$ in a flame dried 2-neck round-bottomed flask, equipped with a stir bar and a septum under argon. Dry THF $(0.43 \mathrm{~mL})$ was added and the clear, slightly yellow solution was immersed into a water-bath at $23{ }^{\circ} \mathrm{C} \pm 1{ }^{\circ} \mathrm{C}$ for $30 \mathrm{~min}$. The 2-iodothiophene $(28 \mu \mathrm{L}, 0.25 \mathrm{mmol})$ was then added and the reaction was stirred for $1 \mathrm{~min}$. A suspension of $\operatorname{Pd}(\mathrm{dba})_{2}(7.2 \mathrm{mg}, 0.0125 \mathrm{mmol})$ in dry THF $(1.0 \mathrm{~mL})$ was added. The initially purple, turbid reaction mixture turns into a clear solution within 30 seconds. Aliquots ( $200 \mathrm{uL}$ were withdrawn after 1, 2, 3, 4, 5, 7, 10, 15, 20, 30, 40, 50, 60, 75, 90, and $120 \mathrm{~min}$. To quench, these samples were added to $100 \mathrm{uL}$ of an aqueous solution of 2dimethylaminoethanethiol hydrochloride $(20 \%)$. The clear colorless to slightly yellow solution was then filtered through a plug of silica-gel, and the silica-gel was washed with diethyl ether to give a total sample volume of $\sim 2 \mathrm{~mL}$. This sample was injected into the GC three times.

initial rate $=0.0154$

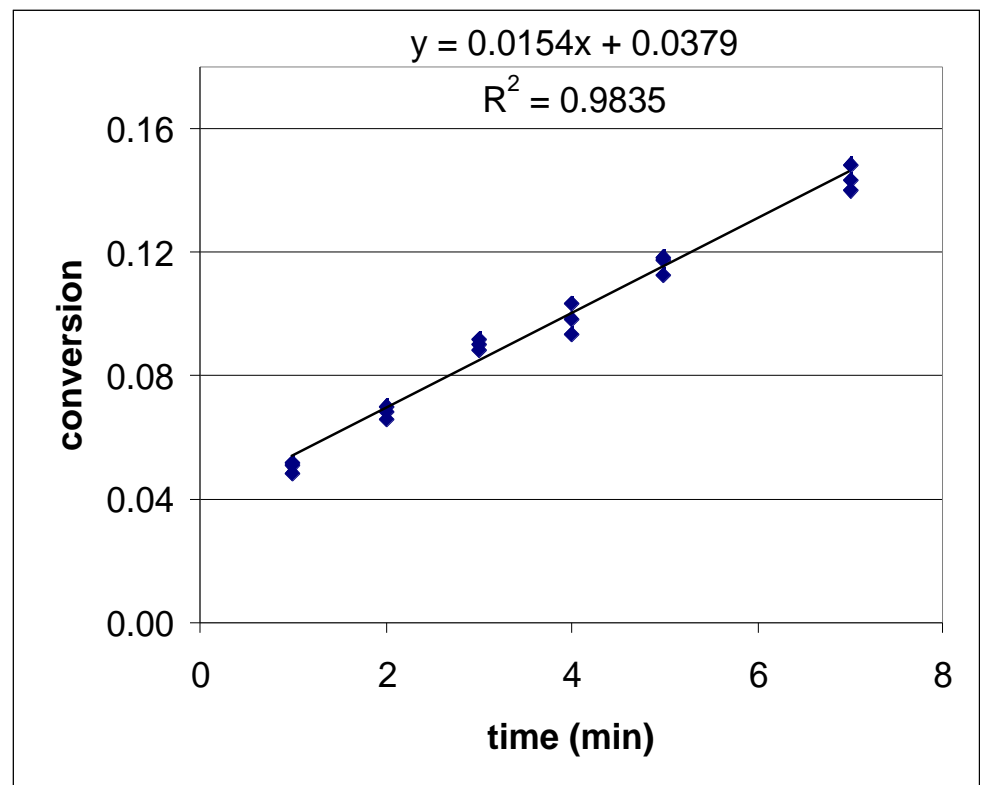




\begin{tabular}{|c|c|c|c|}
\hline time (min) & area $\mathrm{A}$ & area 16 & conversion \\
\hline 1 & 11489 & 1136 & 0.051 \\
\hline 1 & 11435 & 1139 & 0.051 \\
\hline 1 & 10993 & 1022 & 0.048 \\
\hline 2 & 10653 & 1450 & 0.070 \\
\hline 2 & 10580 & 1398 & 0.068 \\
\hline 2 & 10365 & 1321 & 0.066 \\
\hline 3 & 10180 & 1781 & 0.090 \\
\hline 3 & 10529 & 1799 & 0.088 \\
\hline 3 & 10240 & 1817 & 0.092 \\
\hline 4 & 9057 & 1643 & 0.094 \\
\hline 4 & 9618 & 1919 & 0.103 \\
\hline 4 & 9860 & 1872 & 0.098 \\
\hline 5 & 7691 & 1760 & 0.118 \\
\hline 5 & 8663 & 1891 & 0.113 \\
\hline 5 & 8622 & 1966 & 0.118 \\
\hline 7 & 7419 & 2009 & 0.140 \\
\hline 7 & 8229 & 2364 & 0.148 \\
\hline 7 & 7752 & 2150 & 0.143 \\
\hline 10 & 9390 & 3382 & 0.186 \\
\hline 10 & 10263 & 3680 & 0.185 \\
\hline 10 & 10078 & 3607 & 0.185 \\
\hline 15 & 8408 & 3812 & 0.234 \\
\hline 15 & 8033 & 3706 & 0.238 \\
\hline 15 & 7946 & 3478 & 0.226 \\
\hline 20 & 9516 & 5418 & 0.294 \\
\hline 20 & 9754 & 5514 & 0.292 \\
\hline 20 & 9728 & 5490 & 0.292 \\
\hline 30 & 9248 & 7152 & 0.400 \\
\hline 30 & 9186 & 6946 & 0.391 \\
\hline 30 & 9450 & 7168 & 0.392 \\
\hline 40 & 8185 & 7676 & 0.485 \\
\hline 40 & 8655 & 8128 & 0.485 \\
\hline 40 & 8470 & 7832 & 0.478 \\
\hline 50 & 13992 & 15552 & 0.574 \\
\hline 50 & 15225 & 17040 & 0.578 \\
\hline 50 & 12931 & 14476 & 0.578 \\
\hline 60 & 10905 & 14057 & 0.666 \\
\hline 60 & 10273 & 13093 & 0.659 \\
\hline 60 & 11532 & 14698 & 0.658 \\
\hline 75 & 10741 & 16048 & 0.772 \\
\hline 75 & 10618 & 15695 & 0.764 \\
\hline 75 & 10860 & 16374 & 0.779 \\
\hline 90 & 7188 & 11551 & 0.830 \\
\hline 90 & 7638 & 11826 & 0.800 \\
\hline 90 & 7226 & 11687 & 0.836 \\
\hline
\end{tabular}




\section{Order in TBAF, (2.0 equiv).}

(E)-2

2-Iodothiophene

Tetra-n-butylammonium fluoride

Bis(dibenzylideneacetone)palladium $6.25 \mathrm{mM}$ (0.05 equiv)
$125 \mathrm{mM}$ (1.0 equiv)

$125 \mathrm{mM}$ (1.0 equiv)

$250 \mathrm{mM}$ (2.0 equiv)

Following the general procedure silanol $(E)-2(57.2 \mathrm{mg}, 0.25 \mathrm{mmol})$ and biphenyl $(12.2$ $\mathrm{mg}$ ) were dissolved in TBAF solution $(500 \mu \mathrm{L}, 1.0 \mathrm{M}$ in THF, $0.5 \mathrm{mmol})$ in a flame dried 2-neck round-bottomed flask, equipped with a stir bar and a septum under argon. Dry THF $(0.43 \mathrm{~mL})$ was added and the clear, slightly yellow solution was immersed into a water-bath at $23{ }^{\circ} \mathrm{C} \pm 1{ }^{\circ} \mathrm{C}$ for $30 \mathrm{~min}$. The 2-iodothiophene $(28 \mu \mathrm{L}, 0.25 \mathrm{mmol})$ was then added and the reaction was stirred for $1 \mathrm{~min}$. A suspension of $\operatorname{Pd}(\mathrm{dba})_{2}(7.2 \mathrm{mg}, 0.0125 \mathrm{mmol})$ in dry THF $(1.0 \mathrm{~mL})$ was added. The initially purple, turbid reaction mixture turns into a clear solution within 30 seconds. Aliquots ( $200 \mathrm{uL}$ were withdrawn after 1, 2, 3, 4, 5, 7, 10, 15, 20, 30, 40, 50, 60, 75, 90, and $120 \mathrm{~min}$. To quench, these samples were added to $100 \mathrm{uL}$ of an aqueous solution of 2dimethylaminoethanethiol hydrochloride $(20 \%)$. The clear colorless to slightly yellow solution was then filtered through a plug of silica-gel, and the silica-gel was washed with diethyl ether to give a total sample volume of $\sim 2 \mathrm{~mL}$. This sample was injected into the GC three times.

initial rate $=0.0133$

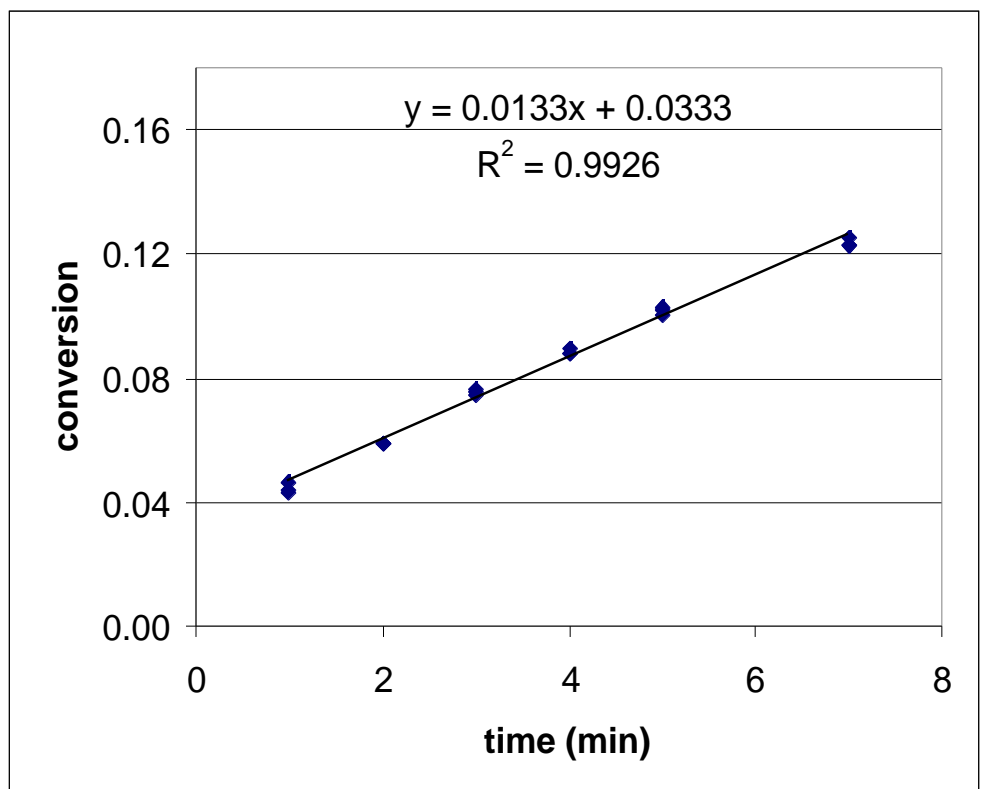




\begin{tabular}{|c|c|c|c|}
\hline time $(\min )$ & area $\mathrm{A}$ & area 16 & conversion \\
\hline 1 & 9680 & 978 & 0.046 \\
\hline 1 & 10005 & 936 & 0.043 \\
\hline 1 & 9696 & 926 & 0.044 \\
\hline 2 & 9831 & 1261 & 0.059 \\
\hline 2 & 9314 & 1188 & 0.059 \\
\hline 2 & 9646 & 1239 & 0.059 \\
\hline 3 & 10247 & 1689 & 0.076 \\
\hline 3 & 11271 & 1826 & 0.075 \\
\hline 3 & 10852 & 1793 & 0.076 \\
\hline 4 & 9785 & 1864 & 0.088 \\
\hline 4 & 9182 & 1758 & 0.088 \\
\hline 4 & 9203 & 1796 & 0.090 \\
\hline 5 & 12074 & 2629 & 0.100 \\
\hline 5 & 12209 & 2725 & 0.103 \\
\hline 5 & 12207 & 2703 & 0.102 \\
\hline 7 & 10832 & 2891 & 0.123 \\
\hline 7 & 11046 & 3016 & 0.126 \\
\hline 7 & 11488 & 3071 & 0.123 \\
\hline 10 & 13620 & 4460 & 0.151 \\
\hline 10 & 13966 & 4574 & 0.151 \\
\hline 10 & 13164 & 4379 & 0.153 \\
\hline 15 & 9764 & 4195 & 0.198 \\
\hline 15 & 9911 & 4212 & 0.196 \\
\hline 15 & 9695 & 4207 & 0.200 \\
\hline 20 & 15035 & 7635 & 0.234 \\
\hline 20 & 15265 & 7944 & 0.239 \\
\hline 20 & 15426 & 7913 & 0.236 \\
\hline 30 & 16760 & 11624 & 0.319 \\
\hline 30 & 19891 & 13756 & 0.318 \\
\hline 30 & 29440 & 20549 & 0.321 \\
\hline 40 & 16384 & 14003 & 0.393 \\
\hline 40 & 14127 & 12069 & 0.393 \\
\hline 40 & 18089 & 15302 & 0.389 \\
\hline 50 & 17594 & 17713 & 0.463 \\
\hline 50 & 16482 & 16691 & 0.466 \\
\hline 50 & 21441 & 21807 & 0.468 \\
\hline 60 & 20902 & 24030 & 0.529 \\
\hline 60 & 12676 & 14553 & 0.528 \\
\hline 60 & 13107 & 15161 & 0.532 \\
\hline 75 & 16681 & 23111 & 0.637 \\
\hline 75 & 18567 & 25622 & 0.635 \\
\hline 75 & 14922 & 20677 & 0.638 \\
\hline 90 & 16667 & 25010 & 0.690 \\
\hline 90 & 21038 & 31621 & 0.692 \\
\hline 90 & 19613 & 29446 & 0.691 \\
\hline 120 & 10409 & 18422 & 0.814 \\
\hline 120 & 42483 & 72513 & 0.785 \\
\hline 120 & 24738 & 41865 & 0.779 \\
\hline
\end{tabular}




\section{Order in TBAF, (4.0 equiv).}

(E)-2

2-Iodothiophene

Tetra-n-butylammonium fluoride $500 \mathrm{mM}$ (4.0 equiv)

Bis(dibenzylideneacetone)palladium $6.25 \mathrm{mM}$ (0.05 equiv)
$125 \mathrm{mM}$ (1.0 equiv)

$125 \mathrm{mM}$ (1.0 equiv)

Following the general procedure silanol $(E)-2(57.2 \mathrm{mg}, 0.25 \mathrm{mmol})$ and biphenyl $(9.8$ $\mathrm{mg}$ ) were dissolved in TBAF solution $(1.0 \mathrm{~mL}, 1.0 \mathrm{M}$ in THF, $1.0 \mathrm{mmol})$ in a flame dried 2-neck round-bottomed flask, equipped with a stir bar and a septum under argon. Dry THF $(0.43 \mathrm{~mL})$ was added and the clear, slightly yellow solution was immersed into a water-bath at $23{ }^{\circ} \mathrm{C} \pm 1{ }^{\circ} \mathrm{C}$ for $30 \mathrm{~min}$. The 2-iodothiophene $(28 \mu \mathrm{L}, 0.25 \mathrm{mmol})$ was then added and the reaction was stirred for $1 \mathrm{~min}$. A suspension of $\operatorname{Pd}(\mathrm{dba})_{2}(7.2 \mathrm{mg}, 0.0125 \mathrm{mmol})$ in dry THF $(0.5 \mathrm{~mL})$ was added. The initially purple, turbid reaction mixture turns into a clear solution within 30 seconds. Aliquots ( $200 \mathrm{uL}$ were withdrawn after 1, 2, 3, 4, 5, 7, 10, 15, 20, 30, 40, 50, 60, 75, 90, and $120 \mathrm{~min}$. To quench, these samples were added to $100 \mathrm{uL}$ of an aqueous solution of 2dimethylaminoethanethiol hydrochloride $(20 \%)$. The clear colorless to slightly yellow solution was then filtered through a plug of silica-gel, and the silica-gel was washed with diethyl ether to give a total sample volume of $\sim 2 \mathrm{~mL}$. This sample was injected into the GC three times.

initial rate $=0.0079$

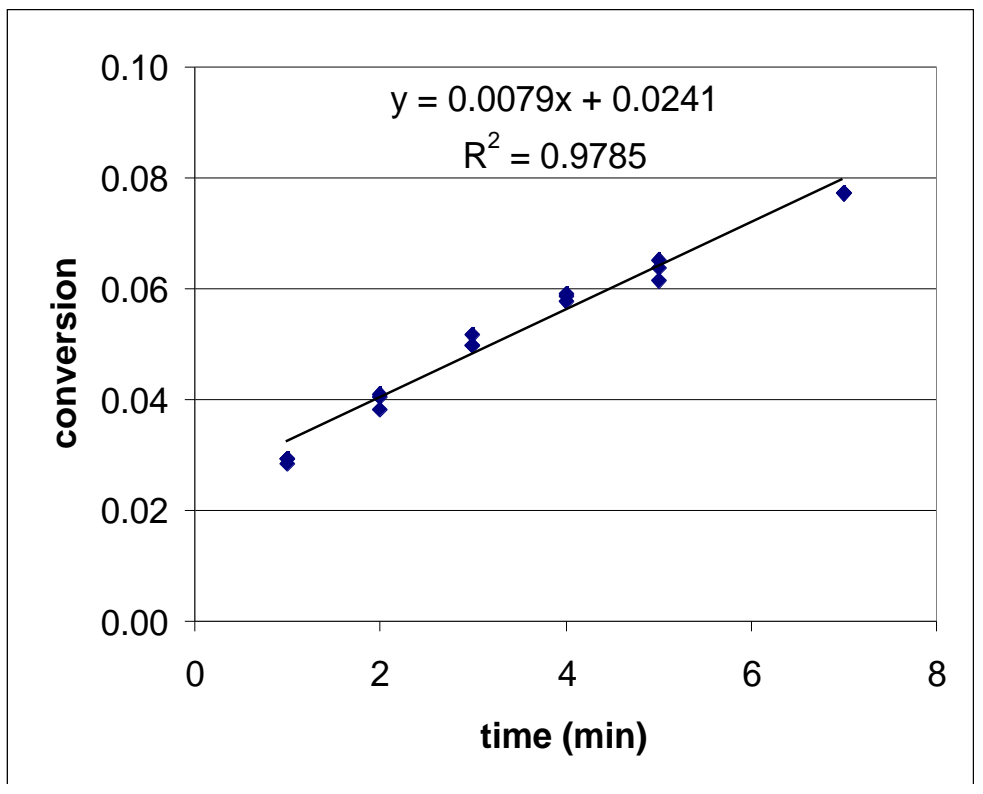




\begin{tabular}{|c|c|c|c|}
\hline time (min) & area $\mathrm{A}$ & area 16 & conversion \\
\hline 1 & 15754 & 1202 & 0.028 \\
\hline 1 & 16273 & 1296 & 0.029 \\
\hline 1 & 16469 & 1316 & 0.030 \\
\hline 2 & 13506 & 1496 & 0.041 \\
\hline 2 & 13575 & 1407 & 0.038 \\
\hline 2 & 13646 & 1486 & 0.040 \\
\hline 3 & 11524 & 1557 & 0.050 \\
\hline 3 & 11751 & 1583 & 0.050 \\
\hline 3 & 11387 & 1588 & 0.052 \\
\hline 4 & 12568 & 1965 & 0.058 \\
\hline 4 & 12380 & 1984 & 0.059 \\
\hline 4 & 12667 & 2001 & 0.058 \\
\hline 5 & 12817 & 2211 & 0.064 \\
\hline 5 & 12284 & 2161 & 0.065 \\
\hline 5 & 12672 & 2113 & 0.062 \\
\hline 7 & 12302 & 2574 & 0.077 \\
\hline 7 & 12391 & 2595 & 0.077 \\
\hline 7 & 12127 & 2535 & 0.077 \\
\hline 10 & 17378 & 4397 & 0.094 \\
\hline 10 & 17235 & 4429 & 0.095 \\
\hline 10 & 17251 & 4450 & 0.095 \\
\hline 15 & 15544 & 5225 & 0.124 \\
\hline 15 & 15566 & 5205 & 0.124 \\
\hline 15 & 16020 & 5262 & 0.121 \\
\hline 20 & 10936 & 4452 & 0.150 \\
\hline 20 & 11167 & 4450 & 0.147 \\
\hline 20 & 10812 & 4335 & 0.148 \\
\hline 30 & 12216 & 6610 & 0.200 \\
\hline 30 & 11890 & 6417 & 0.199 \\
\hline 30 & 12299 & 6477 & 0.195 \\
\hline 40 & 17042 & 11511 & 0.250 \\
\hline 40 & 17468 & 11912 & 0.252 \\
\hline 40 & 17539 & 11824 & 0.249 \\
\hline 50 & 14186 & 11528 & 0.300 \\
\hline 50 & 14565 & 11710 & 0.297 \\
\hline 50 & 14683 & 11600 & 0.292 \\
\hline 60 & 9042 & 8361 & 0.342 \\
\hline 60 & 9395 & 8291 & 0.326 \\
\hline 60 & 9421 & 8457 & 0.332 \\
\hline 75 & 7863 & 8585 & 0.404 \\
\hline 75 & 8424 & 9405 & 0.413 \\
\hline 75 & 8104 & 9052 & 0.413 \\
\hline 90 & 12428 & 16692 & 0.496 \\
\hline 90 & 12621 & 16526 & 0.484 \\
\hline 90 & 13361 & 17298 & 0.478 \\
\hline 120 & 9939 & 15295 & 0.569 \\
\hline 120 & 10743 & 15945 & 0.549 \\
\hline 120 & 10566 & 15287 & 0.535 \\
\hline
\end{tabular}




\section{Order in TBAF, (4.0 equiv).}

(E)-2

2-Iodothiophene

Tetra-n-butylammonium fluoride $500 \mathrm{mM}$ (4.0 equiv)

Bis(dibenzylideneacetone)palladium $6.25 \mathrm{mM}$ (0.05 equiv)
$125 \mathrm{mM}$ (1.0 equiv)

$125 \mathrm{mM}$ (1.0 equiv)

Following the general procedure silanol $(E)-2(57.2 \mathrm{mg}, 0.25 \mathrm{mmol})$ and biphenyl $(8.8$ $\mathrm{mg}$ ) were dissolved in TBAF solution $(1.0 \mathrm{~mL}, 1.0 \mathrm{M}$ in THF, $1.0 \mathrm{mmol})$ in a flame dried 2-neck round-bottomed flask, equipped with a stir bar and a septum under argon. Dry THF $(0.43 \mathrm{~mL})$ was added and the clear, slightly yellow solution was immersed into a water-bath at $23{ }^{\circ} \mathrm{C} \pm 1{ }^{\circ} \mathrm{C}$ for $30 \mathrm{~min}$. The 2-iodothiophene $(28 \mu \mathrm{L}, 0.25 \mathrm{mmol})$ was then added and the reaction was stirred for $1 \mathrm{~min}$. A suspension of $\operatorname{Pd}(\mathrm{dba})_{2}(7.2 \mathrm{mg}, 0.0125 \mathrm{mmol})$ in dry THF $(0.5 \mathrm{~mL})$ was added. The initially purple, turbid reaction mixture turns into a clear solution within 30 seconds. Aliquots ( $200 \mathrm{uL}$ were withdrawn after 1, 2, 3, 4, 5, 7, 10, 15, 20, 30, 40, 50, 60, 75, 90, and $120 \mathrm{~min}$. To quench, these samples were added to $100 \mathrm{uL}$ of an aqueous solution of 2dimethylaminoethanethiol hydrochloride $(20 \%)$. The clear colorless to slightly yellow solution was then filtered through a plug of silica-gel, and the silica-gel was washed with diethyl ether to give a total sample volume of $\sim 2 \mathrm{~mL}$. This sample was injected into the GC three times.

initial rate $=0.0062$

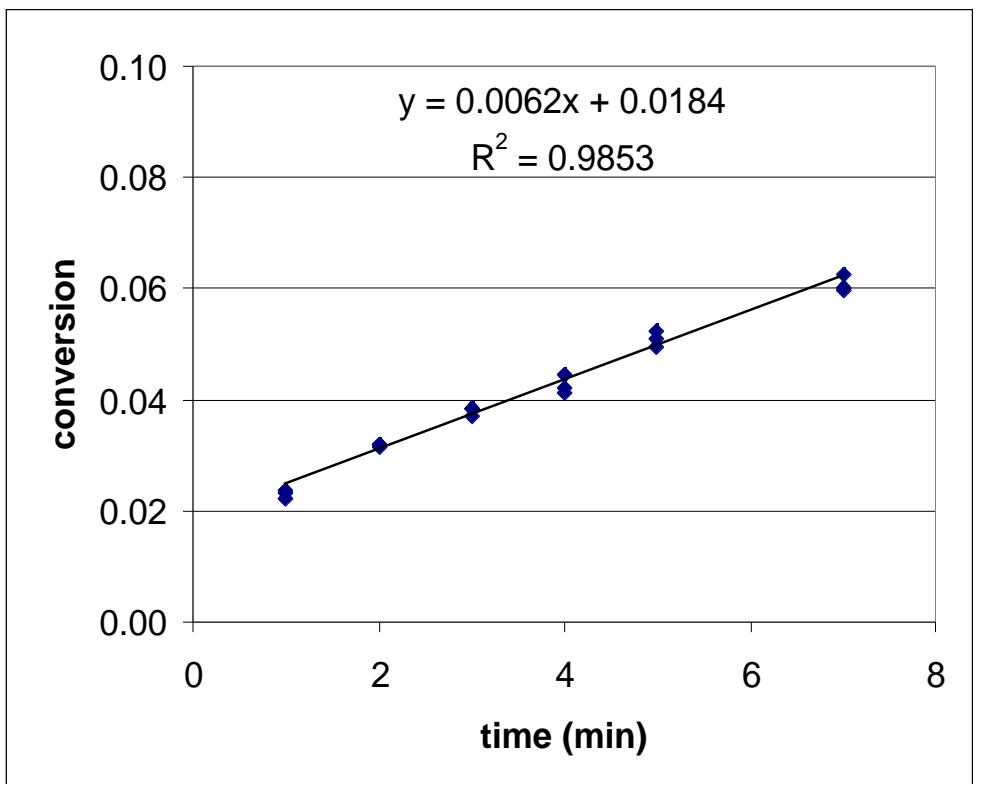




\begin{tabular}{|c|c|c|c|}
\hline time (min) & area $\mathrm{A}$ & area 16 & conversion \\
\hline 1 & 9760 & 687 & 0.023 \\
\hline 1 & 10106 & 717 & 0.024 \\
\hline 1 & 10265 & 683 & 0.022 \\
\hline 2 & 8858 & 843 & 0.032 \\
\hline 2 & 9306 & 899 & 0.032 \\
\hline 2 & 8934 & 866 & 0.032 \\
\hline 3 & 8435 & 941 & 0.037 \\
\hline 3 & 7986 & 922 & 0.038 \\
\hline 3 & 8405 & 976 & 0.039 \\
\hline 4 & 8616 & 1155 & 0.044 \\
\hline 4 & 9139 & 1154 & 0.042 \\
\hline 4 & 8738 & 1090 & 0.041 \\
\hline 5 & 9281 & 1388 & 0.050 \\
\hline 5 & 8922 & 1368 & 0.051 \\
\hline 5 & 9032 & 1421 & 0.052 \\
\hline 7 & 12139 & 2201 & 0.060 \\
\hline 7 & 12343 & 2229 & 0.060 \\
\hline 7 & 11978 & 2259 & 0.063 \\
\hline 10 & 8934 & 1992 & 0.074 \\
\hline 10 & 8809 & 2024 & 0.076 \\
\hline 10 & 8717 & 1994 & 0.076 \\
\hline 15 & 10435 & 2870 & 0.091 \\
\hline 15 & 10987 & 3085 & 0.093 \\
\hline 15 & 10752 & 3014 & 0.093 \\
\hline 20 & 8916 & 2807 & 0.104 \\
\hline 20 & 8616 & 2753 & 0.106 \\
\hline 20 & 8688 & 2819 & 0.108 \\
\hline 30 & 10124 & 4226 & 0.139 \\
\hline 30 & 10731 & 4443 & 0.137 \\
\hline 30 & 10818 & 4460 & 0.137 \\
\hline 40 & 10345 & 5218 & 0.167 \\
\hline 40 & 10200 & 5118 & 0.167 \\
\hline 40 & 10329 & 5150 & 0.165 \\
\hline 50 & 8506 & 4501 & 0.176 \\
\hline 50 & 8669 & 5042 & 0.193 \\
\hline 50 & 8915 & 5138 & 0.191 \\
\hline 60 & 9642 & 6446 & 0.222 \\
\hline 60 & 9218 & 6095 & 0.219 \\
\hline 60 & 9681 & 6537 & 0.224 \\
\hline 75 & 7304 & 5753 & 0.261 \\
\hline 75 & 7589 & 5915 & 0.259 \\
\hline 75 & 7229 & 5712 & 0.262 \\
\hline 90 & 10160 & 9456 & 0.309 \\
\hline 90 & 10155 & 9473 & 0.310 \\
\hline 90 & 9892 & 9146 & 0.307 \\
\hline 120 & 10350 & 12428 & 0.399 \\
\hline 120 & 10413 & 12271 & 0.391 \\
\hline 120 & 10191 & 12334 & 0.402 \\
\hline
\end{tabular}




\section{Order in TBAF, (4.0 equiv).}

(E)-2

2-Iodothiophene

Tetra-n-butylammonium fluoride $500 \mathrm{mM}$ (4.0 equiv)

Bis(dibenzylideneacetone)palladium $6.25 \mathrm{mM}$ (0.05 equiv)
$125 \mathrm{mM}$ (1.0 equiv)

$125 \mathrm{mM}$ (1.0 equiv)

Following the general procedure silanol $(E)-2(57.2 \mathrm{mg}, 0.25 \mathrm{mmol})$ and biphenyl (13.9 $\mathrm{mg}$ ) were dissolved in TBAF solution $(1.0 \mathrm{~mL}, 1.0 \mathrm{M}$ in THF, $1.0 \mathrm{mmol}$ ) in a flame dried 2-neck round-bottomed flask, equipped with a stir bar and a septum under argon. Dry THF $(0.43 \mathrm{~mL})$ was added and the clear, slightly yellow solution was immersed into a water-bath at $23{ }^{\circ} \mathrm{C} \pm 1{ }^{\circ} \mathrm{C}$ for $30 \mathrm{~min}$. The 2-iodothiophene $(28 \mu \mathrm{L}, 0.25 \mathrm{mmol})$ was then added and the reaction was stirred for $1 \mathrm{~min}$. A suspension of $\mathrm{Pd}(\mathrm{dba})_{2}(7.2 \mathrm{mg}, 0.0125 \mathrm{mmol})$ in dry THF $(0.5 \mathrm{~mL})$ was added. The initially purple, turbid reaction mixture turns into a clear solution within 30 seconds. Aliquots ( $200 \mathrm{uL}$ were withdrawn after 1, 2, 3, 4, 5, 7, 10, 15, 20, 30, 40, 50, 60, 75, 90, and $120 \mathrm{~min}$. To quench, these samples were added to $100 \mathrm{uL}$ of an aqueous solution of 2dimethylaminoethanethiol hydrochloride $(20 \%)$. The clear colorless to slightly yellow solution was then filtered through a plug of silica-gel, and the silica-gel was washed with diethyl ether to give a total sample volume of $\sim 2 \mathrm{~mL}$. This sample was injected into the GC three times.

initial rate $=0.0078$

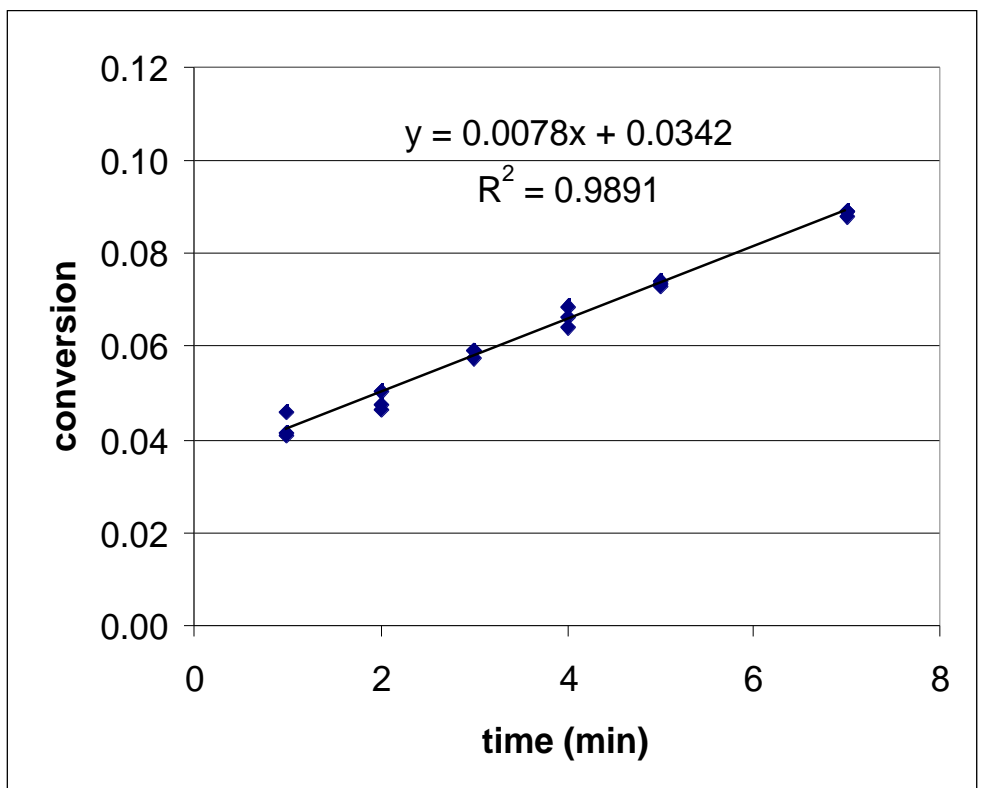




\begin{tabular}{|c|c|c|c|}
\hline time (min) & area $\mathrm{A}$ & area 16 & conversion \\
\hline 1 & 9235 & 733 & 0.042 \\
\hline 1 & 9626 & 749 & 0.041 \\
\hline 1 & 9786 & 853 & 0.046 \\
\hline 2 & 12223 & 1087 & 0.047 \\
\hline 2 & 12629 & 1142 & 0.047 \\
\hline 2 & 12805 & 1223 & 0.050 \\
\hline 3 & 9689 & 1058 & 0.057 \\
\hline 3 & 9290 & 1047 & 0.059 \\
\hline 3 & 9322 & 1055 & 0.059 \\
\hline 4 & 15571 & 2030 & 0.068 \\
\hline 4 & 15834 & 1945 & 0.064 \\
\hline 4 & 16343 & 2065 & 0.066 \\
\hline 5 & 11078 & 1552 & 0.073 \\
\hline 5 & 11057 & 1544 & 0.073 \\
\hline 5 & 11798 & 1665 & 0.074 \\
\hline 7 & 17782 & 3021 & 0.089 \\
\hline 7 & 17822 & 2995 & 0.088 \\
\hline 7 & 17421 & 2963 & 0.089 \\
\hline 10 & 17151 & 3412 & 0.104 \\
\hline 10 & 16910 & 3374 & 0.105 \\
\hline 10 & 16764 & 3422 & 0.107 \\
\hline 15 & 11693 & 2895 & 0.130 \\
\hline 15 & 11844 & 2926 & 0.130 \\
\hline 15 & 11533 & 2859 & 0.130 \\
\hline 20 & 11815 & 3384 & 0.150 \\
\hline 20 & 12029 & 3512 & 0.153 \\
\hline 20 & 12316 & 3505 & 0.149 \\
\hline 30 & 13741 & 4946 & 0.189 \\
\hline 30 & 13655 & 5019 & 0.193 \\
\hline 30 & 13255 & 4879 & 0.193 \\
\hline 40 & 12457 & 5445 & 0.229 \\
\hline 40 & 13323 & 5935 & 0.234 \\
\hline 40 & 12865 & 5702 & 0.232 \\
\hline 50 & 15278 & 7812 & 0.268 \\
\hline 50 & 14598 & 7503 & 0.269 \\
\hline 50 & 14506 & 7528 & 0.272 \\
\hline 60 & 14972 & 8694 & 0.304 \\
\hline 60 & 15007 & 8963 & 0.313 \\
\hline 60 & 15677 & 9206 & 0.308 \\
\hline 75 & 10858 & 7438 & 0.359 \\
\hline 75 & 11192 & 7624 & 0.357 \\
\hline 75 & 10932 & 7629 & 0.366 \\
\hline 90 & 20176 & 16430 & 0.427 \\
\hline 90 & 19637 & 15856 & 0.423 \\
\hline 90 & 19846 & 15671 & 0.414 \\
\hline 120 & 15150 & 15145 & 0.524 \\
\hline 120 & 14718 & 15008 & 0.535 \\
\hline 120 & 14345 & 14757 & 0.539 \\
\hline
\end{tabular}




\section{Order in TBAF, (5.0 equiv).}

(E)-2

2-Iodothiophene

Tetra-n-butylammonium fluoride

Bis(dibenzylideneacetone)palladium $6.25 \mathrm{mM}$ (0.05 equiv)
$125 \mathrm{mM}$ (1.0 equiv)

$125 \mathrm{mM}$ ( 1.0 equiv)

$625 \mathrm{mM}$ (5.0 equiv)

Following the general procedure silanol $(E)-2(57.2 \mathrm{mg}, 0.25 \mathrm{mmol})$ and biphenyl $(8.6$ $\mathrm{mg}$ ) were dissolved in TBAF solution (1.25 mL, 1.0 M in THF, $1.25 \mathrm{mmol})$ in a flame dried 2neck round-bottomed flask, equipped with a stir bar and a septum under argon. Dry THF (0.18 $\mathrm{mL}$ ) was added and the clear, slightly yellow solution was immersed into a water-bath at $23{ }^{\circ} \mathrm{C} \pm$ $1{ }^{\circ} \mathrm{C}$ for $30 \mathrm{~min}$. The 2-iodothiophene $(28 \mu \mathrm{L}, 0.25 \mathrm{mmol})$ was then added and the reaction was stirred for $1 \mathrm{~min}$. A suspension of $\operatorname{Pd}(\mathrm{dba})_{2}(7.2 \mathrm{mg}, 0.0125 \mathrm{mmol})$ in dry THF $(0.5 \mathrm{~mL})$ was added. The initially purple, turbid reaction mixture turns into a clear solution within 30 seconds. Aliquots ( $200 \mathrm{uL}$ were withdrawn after 1, 2, 3, 4, 5, 7, 10, 15, 20, 30, 40, 50, 60, 75, 90, and $120 \mathrm{~min}$. To quench, these samples were added to $100 \mathrm{uL}$ of an aqueous solution of 2dimethylaminoethanethiol hydrochloride $(20 \%)$. The clear colorless to slightly yellow solution was then filtered through a plug of silica-gel, and the silica-gel was washed with diethyl ether to give a total sample volume of $\sim 2 \mathrm{~mL}$. This sample was injected into the GC three times.

initial rate $=0.0044$

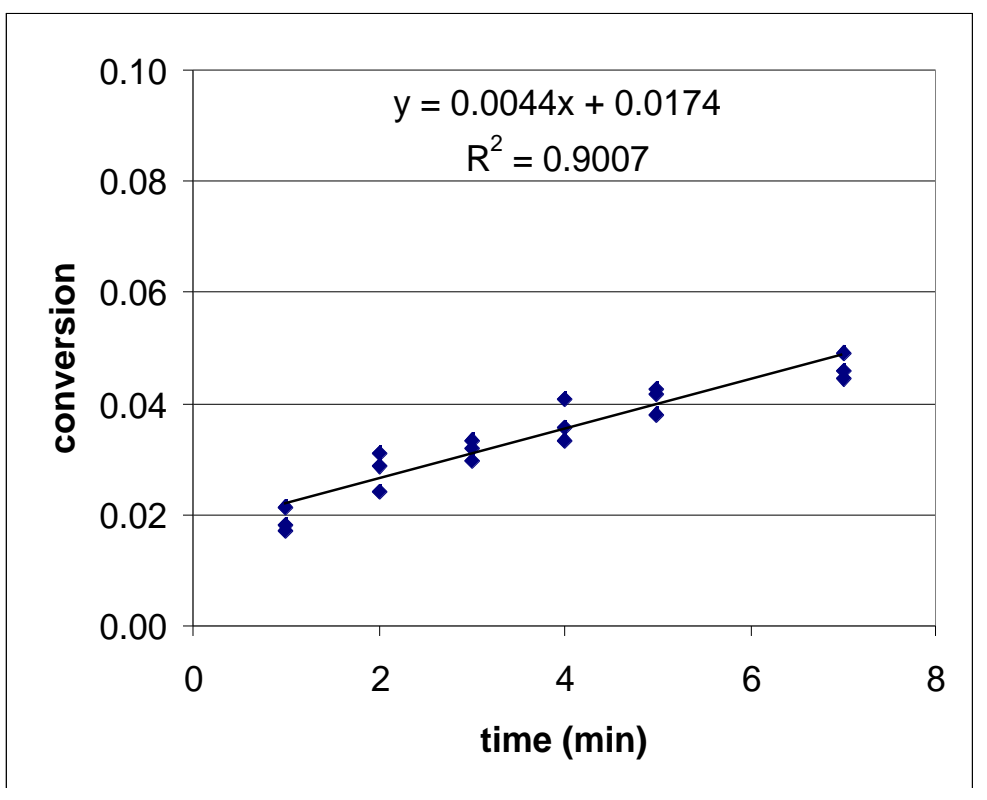




\begin{tabular}{|c|c|c|c|}
\hline time $(\min )$ & area $\mathrm{A}$ & area 16 & conversion \\
\hline 1 & 15323 & 1003 & 0.021 \\
\hline 1 & 13646 & 730 & 0.017 \\
\hline 1 & 13482 & 748 & 0.018 \\
\hline 2 & 7402 & 545 & 0.024 \\
\hline 2 & 7392 & 703 & 0.031 \\
\hline 2 & 13536 & 1189 & 0.028 \\
\hline 3 & 6277 & 644 & 0.033 \\
\hline 3 & 6181 & 608 & 0.032 \\
\hline 3 & 6328 & 578 & 0.030 \\
\hline 4 & 6548 & 672 & 0.033 \\
\hline 4 & 12390 & 1364 & 0.036 \\
\hline 4 & 6435 & 806 & 0.041 \\
\hline 5 & 7372 & 858 & 0.038 \\
\hline 5 & 7223 & 933 & 0.042 \\
\hline 5 & 7036 & 922 & 0.043 \\
\hline 7 & 9244 & 1395 & 0.049 \\
\hline 7 & 9180 & 1255 & 0.044 \\
\hline 7 & 9301 & 1312 & 0.046 \\
\hline 10 & 6726 & 1305 & 0.063 \\
\hline 10 & 6682 & 1313 & 0.064 \\
\hline 10 & 6843 & 1306 & 0.062 \\
\hline 15 & 7262 & 1745 & 0.078 \\
\hline 15 & 7578 & 1878 & 0.080 \\
\hline 15 & 8597 & 1881 & 0.071 \\
\hline 20 & 11536 & 3144 & 0.088 \\
\hline 20 & 10010 & 2943 & 0.095 \\
\hline 20 & 10098 & 2808 & 0.090 \\
\hline 30 & 6463 & 2364 & 0.119 \\
\hline 30 & 6623 & 2284 & 0.112 \\
\hline 30 & 6516 & 2456 & 0.122 \\
\hline 40 & 8596 & 3939 & 0.149 \\
\hline 40 & 8704 & 4074 & 0.152 \\
\hline 40 & 8461 & 3696 & 0.142 \\
\hline 50 & 8390 & 4949 & 0.191 \\
\hline 50 & 8963 & 5095 & 0.184 \\
\hline 50 & 8968 & 4827 & 0.175 \\
\hline 60 & 9750 & 6340 & 0.211 \\
\hline 60 & 8786 & 5746 & 0.212 \\
\hline 60 & 8658 & 5698 & 0.213 \\
\hline 75 & 10185 & 8045 & 0.256 \\
\hline 75 & 10411 & 8162 & 0.254 \\
\hline 75 & 10093 & 7832 & 0.252 \\
\hline 90 & 7097 & 5901 & 0.270 \\
\hline 90 & 7221 & 6404 & 0.288 \\
\hline 90 & 7109 & 6402 & 0.292 \\
\hline 120 & 6270 & 7203 & 0.373 \\
\hline 120 & 6281 & 6684 & 0.345 \\
\hline 120 & 6169 & 7488 & 0.394 \\
\hline
\end{tabular}




\section{Order in TBAF, (5.0 equiv).}

(E)-2

2-Iodothiophene

Tetra-n-butylammonium fluoride

Bis(dibenzylideneacetone)palladium $6.25 \mathrm{mM}$ (0.05 equiv)
$125 \mathrm{mM}$ (1.0 equiv)

$125 \mathrm{mM}$ (1.0 equiv)

$625 \mathrm{mM}$ (5.0 equiv)

Following the general procedure silanol $(E)-2(57.2 \mathrm{mg}, 0.25 \mathrm{mmol})$ and biphenyl $(11.4$ $\mathrm{mg}$ ) were dissolved in TBAF solution (1.25 mL, 1.0 M in THF, $1.25 \mathrm{mmol})$ in a flame dried 2neck round-bottomed flask, equipped with a stir bar and a septum under argon. Dry THF (0.18 $\mathrm{mL}$ ) was added and the clear, slightly yellow solution was immersed into a water-bath at $23^{\circ} \mathrm{C} \pm$ $1{ }^{\circ} \mathrm{C}$ for $30 \mathrm{~min}$. The 2-iodothiophene $(28 \mu \mathrm{L}, 0.25 \mathrm{mmol})$ was then added and the reaction was stirred for $1 \mathrm{~min}$. A suspension of $\mathrm{Pd}(\mathrm{dba})_{2}(7.2 \mathrm{mg}, 0.0125 \mathrm{mmol})$ in dry THF $(0.5 \mathrm{~mL})$ was added. The initially purple, turbid reaction mixture turns into a clear solution within 30 seconds. Aliquots ( $200 \mathrm{uL}$ were withdrawn after 1, 2, 3, 4, 5, 7, 10, 15, 20, 30, 40, 50, 60, 75, 90, and $120 \mathrm{~min}$. To quench, these samples were added to $100 \mathrm{uL}$ of an aqueous solution of 2dimethylaminoethanethiol hydrochloride $(20 \%)$. The clear colorless to slightly yellow solution was then filtered through a plug of silica-gel, and the silica-gel was washed with diethyl ether to give a total sample volume of $\sim 2 \mathrm{~mL}$. This sample was injected into the GC three times.

initial rate $=0.0052$

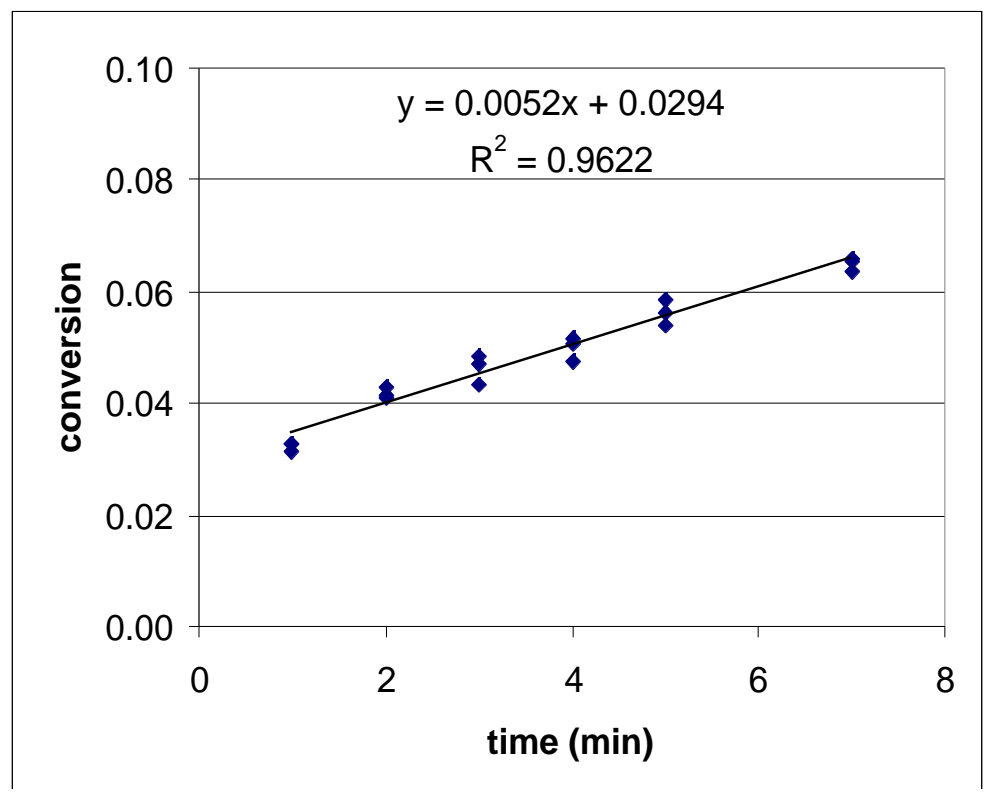




\begin{tabular}{|c|c|c|c|}
\hline time (min) & area $\mathrm{A}$ & area 16 & conversion \\
\hline 1 & 13292 & 1012 & 0.033 \\
\hline 1 & 14520 & 1053 & 0.031 \\
\hline 1 & 15832 & 1202 & 0.033 \\
\hline 2 & 43313 & 4123 & 0.041 \\
\hline 2 & 27896 & 2691 & 0.041 \\
\hline 2 & 31239 & 3104 & 0.043 \\
\hline 3 & 22437 & 2522 & 0.048 \\
\hline 3 & 53479 & 5844 & 0.047 \\
\hline 3 & 62189 & 6299 & 0.044 \\
\hline 4 & 42035 & 4639 & 0.047 \\
\hline 4 & 94258 & 11071 & 0.050 \\
\hline 4 & 119780 & 14338 & 0.051 \\
\hline 5 & 26202 & 3285 & 0.054 \\
\hline 5 & 22043 & 2886 & 0.056 \\
\hline 5 & 28727 & 3900 & 0.058 \\
\hline 7 & 17438 & 2675 & 0.066 \\
\hline 7 & 18097 & 2671 & 0.063 \\
\hline 7 & 17981 & 2744 & 0.066 \\
\hline 10 & 14910 & 2582 & 0.074 \\
\hline 10 & 15334 & 2499 & 0.070 \\
\hline 10 & 14729 & 2412 & 0.070 \\
\hline 15 & 19110 & 3826 & 0.086 \\
\hline 15 & 19549 & 3845 & 0.085 \\
\hline 15 & 19417 & 3761 & 0.083 \\
\hline 20 & 14152 & 3350 & 0.102 \\
\hline 20 & 14403 & 3276 & 0.098 \\
\hline 20 & 15020 & 3599 & 0.103 \\
\hline 30 & 17792 & 5702 & 0.138 \\
\hline 30 & 17023 & 5403 & 0.136 \\
\hline 30 & 17800 & 5602 & 0.135 \\
\hline 40 & 13775 & 5064 & 0.158 \\
\hline 40 & 13735 & 5211 & 0.163 \\
\hline 40 & 13786 & 5157 & 0.161 \\
\hline 50 & 17445 & 7944 & 0.196 \\
\hline 50 & 17039 & 7888 & 0.199 \\
\hline 50 & 17492 & 7764 & 0.191 \\
\hline 60 & 13940 & 7191 & 0.222 \\
\hline 60 & 14500 & 7309 & 0.217 \\
\hline 60 & 14287 & 7300 & 0.220 \\
\hline 75 & 8432 & 4798 & 0.245 \\
\hline 75 & 8314 & 5033 & 0.260 \\
\hline 75 & 8114 & 4927 & 0.261 \\
\hline 90 & 7643 & 5228 & 0.294 \\
\hline 90 & 7672 & 5292 & 0.297 \\
\hline 90 & 7702 & 5247 & 0.293 \\
\hline
\end{tabular}




\section{Order in TBAF, (5.0 equiv).}

(E)-2

2-Iodothiophene

Tetra-n-butylammonium fluoride

Bis(dibenzylideneacetone)palladium $6.25 \mathrm{mM}$ (0.05 equiv)
$125 \mathrm{mM}$ (1.0 equiv)

$125 \mathrm{mM}$ (1.0 equiv)

$625 \mathrm{mM}$ (5.0 equiv)

Following the general procedure silanol $(E)-2(57.2 \mathrm{mg}, 0.25 \mathrm{mmol})$ and biphenyl $(7.2$ $\mathrm{mg}$ ) were dissolved in TBAF solution $(1.25 \mathrm{~mL}, 1.0 \mathrm{M}$ in THF, $1.25 \mathrm{mmol})$ in a flame dried 2neck round-bottomed flask, equipped with a stir bar and a septum under argon. Dry THF (0.18 $\mathrm{mL}$ ) was added and the clear, slightly yellow solution was immersed into a water-bath at $23{ }^{\circ} \mathrm{C} \pm$ $1{ }^{\circ} \mathrm{C}$ for $30 \mathrm{~min}$. The 2-iodothiophene $(28 \mu \mathrm{L}, 0.25 \mathrm{mmol})$ was then added and the reaction was stirred for $1 \mathrm{~min}$. A suspension of $\operatorname{Pd}(\mathrm{dba})_{2}(7.2 \mathrm{mg}, 0.0125 \mathrm{mmol})$ in dry THF $(0.5 \mathrm{~mL})$ was added. The initially purple, turbid reaction mixture turns into a clear solution within 30 seconds. Aliquots ( $200 \mathrm{uL}$ were withdrawn after 1, 2, 3, 4, 5, 7, 10, 15, 20, 30, 40, 50, 60, 75, 90, and $120 \mathrm{~min}$. To quench, these samples were added to $100 \mathrm{uL}$ of an aqueous solution of 2dimethylaminoethanethiol hydrochloride $(20 \%)$. The clear colorless to slightly yellow solution was then filtered through a plug of silica-gel, and the silica-gel was washed with diethyl ether to give a total sample volume of $\sim 2 \mathrm{~mL}$. This sample was injected into the GC three times.

initial rate $=0.0065$

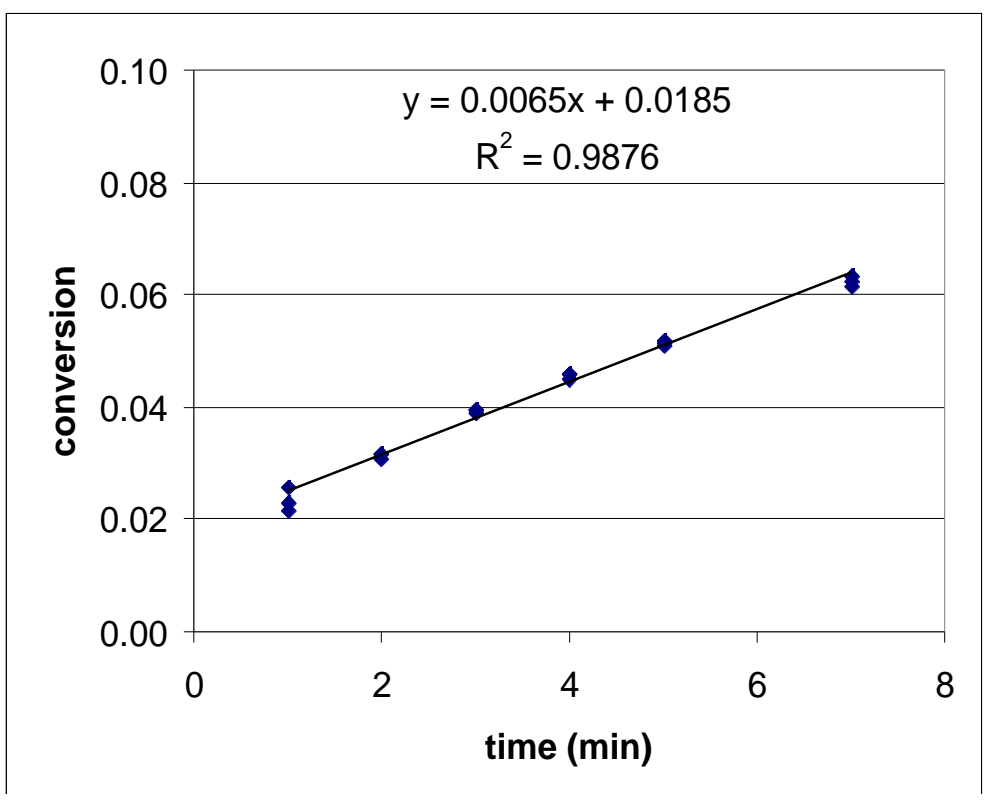




\begin{tabular}{|c|c|c|c|}
\hline time (min) & area $\mathrm{A}$ & area $\mathbf{1 6}$ & conversion \\
\hline 1 & 28124 & 2245 & 0.022 \\
\hline 1 & 9028 & 847 & 0.025 \\
\hline 1 & 14067 & 1182 & 0.023 \\
\hline 2 & 14134 & 1603 & 0.031 \\
\hline 2 & 8972 & 1048 & 0.032 \\
\hline 2 & 8271 & 962 & 0.032 \\
\hline 3 & 6392 & 933 & 0.040 \\
\hline 3 & 6274 & 914 & 0.040 \\
\hline 3 & 6161 & 885 & 0.039 \\
\hline 4 & 6095 & 1007 & 0.045 \\
\hline 4 & 5907 & 995 & 0.046 \\
\hline 4 & 5653 & 958 & 0.046 \\
\hline 5 & 8588 & 1631 & 0.052 \\
\hline 5 & 8174 & 1555 & 0.052 \\
\hline 5 & 8147 & 1526 & 0.051 \\
\hline 7 & 7382 & 1719 & 0.063 \\
\hline 7 & 7219 & 1654 & 0.062 \\
\hline 7 & 8022 & 1819 & 0.062 \\
\hline 10 & 8810 & 2417 & 0.074 \\
\hline 10 & 8133 & 2113 & 0.071 \\
\hline 10 & 9405 & 2558 & 0.074 \\
\hline 15 & 8641 & 2943 & 0.092 \\
\hline 15 & 8167 & 2782 & 0.092 \\
\hline 15 & 9541 & 3200 & 0.091 \\
\hline 20 & 8619 & 3421 & 0.108 \\
\hline 20 & 9847 & 3988 & 0.110 \\
\hline 20 & 12687 & 4948 & 0.106 \\
\hline 30 & 11449 & 5705 & 0.135 \\
\hline 30 & 20634 & 10155 & 0.134 \\
\hline 30 & 20080 & 9857 & 0.133 \\
\hline 40 & 23435 & 14485 & 0.168 \\
\hline 40 & 10792 & 6691 & 0.168 \\
\hline 40 & 10792 & 6691 & 0.168 \\
\hline 50 & 14452 & 10189 & 0.191 \\
\hline 50 & 19008 & 13516 & 0.193 \\
\hline 50 & 7400 & 5301 & 0.195 \\
\hline 60 & 15637 & 12708 & 0.221 \\
\hline 60 & 15811 & 13059 & 0.224 \\
\hline 60 & 12761 & 10410 & 0.222 \\
\hline 75 & 10933 & 10360 & 0.257 \\
\hline 75 & 17852 & 16821 & 0.256 \\
\hline 75 & 14921 & 14384 & 0.262 \\
\hline 90 & 25877 & 29619 & 0.311 \\
\hline 90 & 18178 & 21036 & 0.314 \\
\hline 90 & 17582 & 19939 & 0.308 \\
\hline 120 & 19806 & 27472 & 0.377 \\
\hline 120 & 134004 & 175336 & 0.355 \\
\hline 120 & 21458 & 29576 & 0.374 \\
\hline
\end{tabular}




\section{Order in TBAF, (7.0 equiv).}

(E)-2

2-Iodothiophene

Tetra-n-butylammonium fluoride

Bis(dibenzylideneacetone)palladium $6.25 \mathrm{mM}$ (0.05 equiv)
$125 \mathrm{mM}$ (1.0 equiv)

$125 \mathrm{mM}$ (1.0 equiv)

$875 \mathrm{mM}$ (7.0 equiv)

Following the general procedure silanol $(E)-2(57.2 \mathrm{mg}, 0.25 \mathrm{mmol})$ and biphenyl $(6.3$ $\mathrm{mg}$ ) were dissolved in TBAF solution $(1.75 \mathrm{~mL}, 1.0 \mathrm{M}$ in THF, $1.75 \mathrm{mmol})$ in a flame dried 2neck round-bottomed flask, equipped with a stir bar and a septum under argon. The clear, slightly yellow solution was immersed into a water-bath at $23{ }^{\circ} \mathrm{C} \pm 1{ }^{\circ} \mathrm{C}$ for $30 \mathrm{~min}$. The 2iodothiophene $(28 \mu \mathrm{L}, 0.25 \mathrm{mmol})$ was then added and the reaction was stirred for $1 \mathrm{~min}$. A suspension of $\mathrm{Pd}(\mathrm{dba})_{2}(7.2 \mathrm{mg}, 0.0125 \mathrm{mmol})$ in dry THF $(0.18 \mathrm{~mL})$ was added. The initially purple, turbid reaction mixture turns into a clear solution within 30 seconds. Aliquots $(\sim 200 \mathrm{uL}$ were withdrawn after $1,2,3,4,5,7,10,15,20,30,40,50,60,75,90$, and $120 \mathrm{~min}$. To quench, these samples were added to $100 \mathrm{uL}$ of an aqueous solution of 2-dimethylaminoethanethiol hydrochloride $(20 \%)$. The clear colorless to slightly yellow solution was then filtered through a plug of silica-gel, and the silica-gel was washed with diethyl ether to give a total sample volume of $\sim 2 \mathrm{~mL}$. This sample was injected into the GC three times.

initial rate $=0.0029$

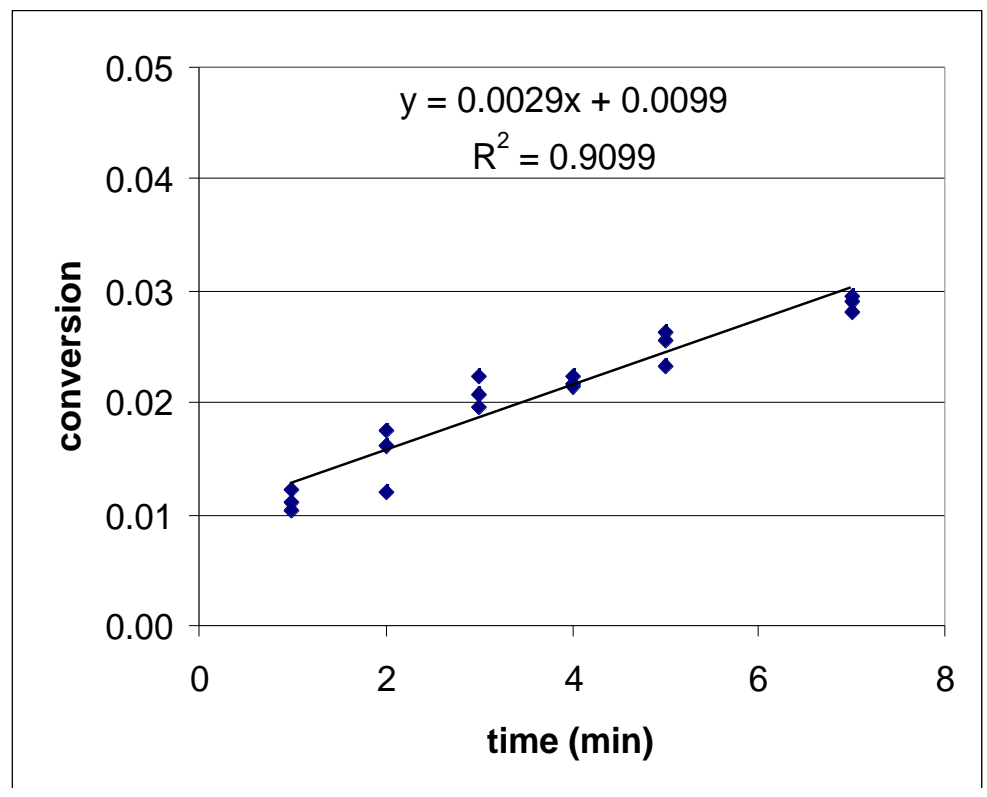




\begin{tabular}{|c|c|c|c|}
\hline time $(\min )$ & area $\mathrm{A}$ & area $\mathbf{1 6}$ & conversion \\
\hline 1 & 14439 & 679 & 0.011 \\
\hline 1 & 14183 & 613 & 0.010 \\
\hline 1 & 15195 & 787 & 0.012 \\
\hline 2 & 8170 & 606 & 0.018 \\
\hline 2 & 8011 & 545 & 0.016 \\
\hline 2 & 7884 & 397 & 0.012 \\
\hline 3 & 7159 & 670 & 0.022 \\
\hline 3 & 7399 & 647 & 0.021 \\
\hline 3 & 6952 & 570 & 0.019 \\
\hline 4 & 6481 & 587 & 0.022 \\
\hline 4 & 6663 & 607 & 0.022 \\
\hline 4 & 6425 & 603 & 0.022 \\
\hline 5 & 6222 & 611 & 0.023 \\
\hline 5 & 7063 & 758 & 0.025 \\
\hline 5 & 7289 & 803 & 0.026 \\
\hline 7 & 7315 & 893 & 0.029 \\
\hline 7 & 6386 & 795 & 0.030 \\
\hline 7 & 6518 & 771 & 0.028 \\
\hline 10 & 9038 & 1295 & 0.034 \\
\hline 10 & 8376 & 1213 & 0.034 \\
\hline 10 & 8938 & 1303 & 0.035 \\
\hline 15 & 9357 & 1728 & 0.044 \\
\hline 15 & 9646 & 1751 & 0.043 \\
\hline 15 & 9886 & 1827 & 0.044 \\
\hline 20 & 7622 & 1753 & 0.055 \\
\hline 20 & 7668 & 1737 & 0.054 \\
\hline 20 & 7915 & 1857 & 0.056 \\
\hline 30 & 6726 & 1940 & 0.069 \\
\hline 30 & 6301 & 1820 & 0.069 \\
\hline 30 & 6237 & 1896 & 0.072 \\
\hline 40 & 7649 & 2575 & 0.080 \\
\hline 40 & 7735 & 2661 & 0.082 \\
\hline 40 & 7516 & 2595 & 0.082 \\
\hline 50 & 7036 & 3086 & 0.104 \\
\hline 50 & 7103 & 3137 & 0.105 \\
\hline 50 & 7487 & 3278 & 0.104 \\
\hline 60 & 8224 & 4270 & 0.123 \\
\hline 60 & 8556 & 4480 & 0.124 \\
\hline 60 & 8279 & 4388 & 0.126 \\
\hline 75 & 6424 & 4097 & 0.152 \\
\hline 75 & 6418 & 4215 & 0.156 \\
\hline 75 & 6686 & 3950 & 0.140 \\
\hline
\end{tabular}




\section{Order in TBAF, (7.0 equiv).}

(E)-2

2-Iodothiophene

Tetra-n-butylammonium fluoride

Bis(dibenzylideneacetone)palladium $6.25 \mathrm{mM}$ (0.05 equiv)
$125 \mathrm{mM}$ (1.0 equiv)

$125 \mathrm{mM}$ (1.0 equiv)

$875 \mathrm{mM}$ (7.0 equiv)

Following the general procedure silanol $(E)-2(57.2 \mathrm{mg}, 0.25 \mathrm{mmol})$ and biphenyl $(7.6$ $\mathrm{mg}$ ) were dissolved in TBAF solution $(1.75 \mathrm{~mL}, 1.0 \mathrm{M}$ in THF, $1.75 \mathrm{mmol})$ in a flame dried 2neck round-bottomed flask, equipped with a stir bar and a septum under argon. The clear, slightly yellow solution was immersed into a water-bath at $23{ }^{\circ} \mathrm{C} \pm 1{ }^{\circ} \mathrm{C}$ for $30 \mathrm{~min}$. The 2iodothiophene $(28 \mu \mathrm{L}, 0.25 \mathrm{mmol})$ was then added and the reaction was stirred for $1 \mathrm{~min}$. A suspension of $\operatorname{Pd}(\mathrm{dba})_{2}(7.2 \mathrm{mg}, 0.0125 \mathrm{mmol})$ in dry THF $(0.18 \mathrm{~mL})$ was added. The initially purple, turbid reaction mixture turns into a clear solution within 30 seconds. Aliquots $(\sim 200 \mathrm{uL}$ were withdrawn after $1,2,3,4,5,7,10,15,20,30,40,50,60,75,90$, and $120 \mathrm{~min}$. To quench, these samples were added to $100 \mathrm{uL}$ of an aqueous solution of 2-dimethylaminoethanethiol hydrochloride $(20 \%)$. The clear colorless to slightly yellow solution was then filtered through a plug of silica-gel, and the silica-gel was washed with diethyl ether to give a total sample volume of $\sim 2 \mathrm{~mL}$. This sample was injected into the GC three times.

initial rate $=0.0042$

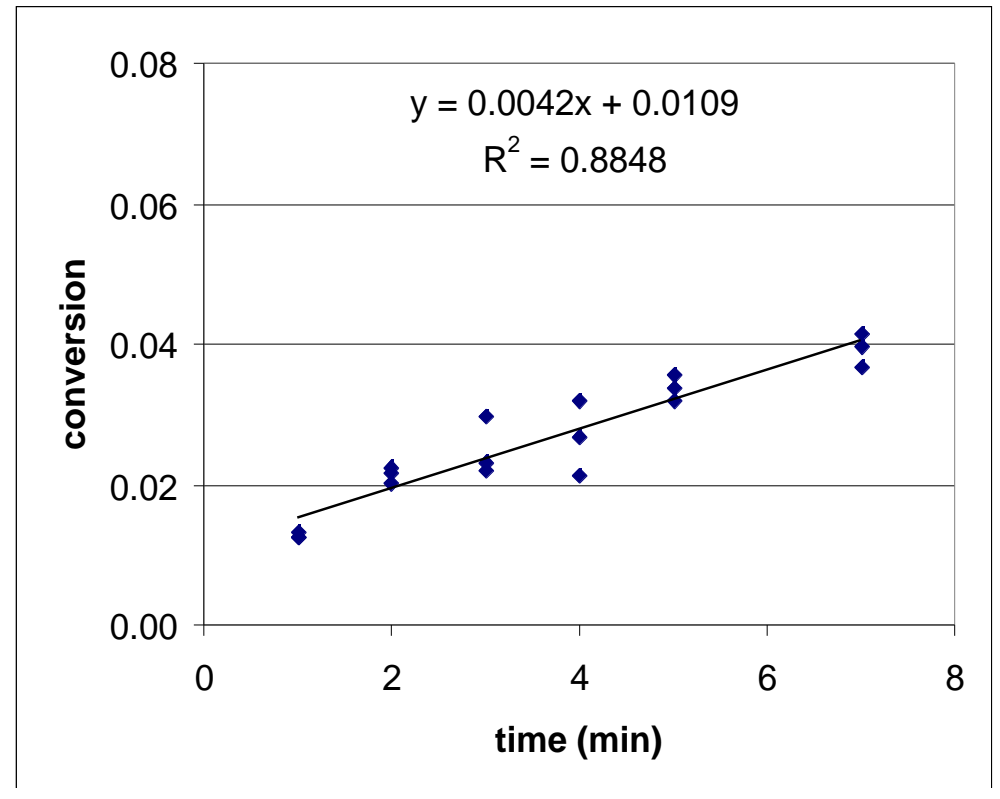




\begin{tabular}{|c|c|c|c|}
\hline time (min) & area $\mathrm{A}$ & area 16 & conversion \\
\hline 1 & 13322 & 579 & 0.012 \\
\hline 1 & 12497 & 547 & 0.013 \\
\hline 1 & 12294 & 565 & 0.013 \\
\hline 2 & 11270 & 846 & 0.022 \\
\hline 2 & 14879 & 1165 & 0.022 \\
\hline 2 & 11089 & 779 & 0.020 \\
\hline 3 & 12923 & 1333 & 0.030 \\
\hline 3 & 18323 & 1415 & 0.022 \\
\hline 3 & 13001 & 1052 & 0.023 \\
\hline 4 & 11817 & 1311 & 0.032 \\
\hline 4 & 12141 & 1139 & 0.027 \\
\hline 4 & 16714 & 1237 & 0.021 \\
\hline 5 & 9222 & 1022 & 0.032 \\
\hline 5 & 9128 & 1132 & 0.036 \\
\hline 5 & 9720 & 1144 & 0.034 \\
\hline 7 & 10466 & 1339 & 0.037 \\
\hline 7 & 14169 & 1957 & 0.040 \\
\hline 7 & 11150 & 1620 & 0.042 \\
\hline 10 & 16046 & 3002 & 0.054 \\
\hline 10 & 14629 & 2756 & 0.054 \\
\hline 10 & 15013 & 2468 & 0.047 \\
\hline 15 & 14421 & 3252 & 0.065 \\
\hline 15 & 14468 & 3253 & 0.064 \\
\hline 15 & 14208 & 3586 & 0.072 \\
\hline 20 & 9811 & 2900 & 0.085 \\
\hline 20 & 9710 & 2970 & 0.088 \\
\hline 20 & 10423 & 2791 & 0.077 \\
\hline 30 & 14187 & 4267 & 0.086 \\
\hline 30 & 18981 & 5498 & 0.083 \\
\hline 30 & 17835 & 5681 & 0.091 \\
\hline 40 & 18801 & 6469 & 0.099 \\
\hline 40 & 15571 & 5902 & 0.109 \\
\hline 40 & 16411 & 7080 & 0.124 \\
\hline 50 & 9512 & 4714 & 0.142 \\
\hline 50 & 9635 & 3742 & 0.111 \\
\hline 50 & 10265 & 4661 & 0.130 \\
\hline 60 & 18005 & 8825 & 0.140 \\
\hline 60 & 21068 & 9736 & 0.132 \\
\hline 60 & 20473 & 9477 & 0.133 \\
\hline 75 & 13403 & 7339 & 0.157 \\
\hline 75 & 18589 & 10069 & 0.155 \\
\hline 75 & 10524 & 6121 & 0.167 \\
\hline 90 & 15499 & 10019 & 0.185 \\
\hline 90 & 23568 & 15273 & 0.186 \\
\hline 90 & 15414 & 9805 & 0.182 \\
\hline 120 & 23905 & 18954 & 0.227 \\
\hline 120 & 24823 & 19941 & 0.230 \\
\hline 120 & 27705 & 22002 & 0.228 \\
\hline
\end{tabular}




\section{Order in TBAF, (7.0 equiv).}

(E)-2

2-Iodothiophene

Tetra-n-butylammonium fluoride

Bis(dibenzylideneacetone)palladium $6.25 \mathrm{mM}$ (0.05 equiv)
$125 \mathrm{mM}$ (1.0 equiv)

$125 \mathrm{mM}$ (1.0 equiv)

$875 \mathrm{mM}$ (7.0 equiv)

Following the general procedure silanol $(E)-2(57.2 \mathrm{mg}, 0.25 \mathrm{mmol})$ and biphenyl $(6.5$ $\mathrm{mg}$ ) were dissolved in TBAF solution $(1.75 \mathrm{~mL}, 1.0 \mathrm{M}$ in THF, $1.75 \mathrm{mmol})$ in a flame dried 2neck round-bottomed flask, equipped with a stir bar and a septum under argon. The clear, slightly yellow solution was immersed into a water-bath at $23{ }^{\circ} \mathrm{C} \pm 1{ }^{\circ} \mathrm{C}$ for $30 \mathrm{~min}$. The 2iodothiophene $(28 \mu \mathrm{L}, 0.25 \mathrm{mmol})$ was then added and the reaction was stirred for $1 \mathrm{~min}$. A suspension of $\mathrm{Pd}(\mathrm{dba})_{2}(7.2 \mathrm{mg}, 0.0125 \mathrm{mmol})$ in dry THF $(0.18 \mathrm{~mL})$ was added. The initially purple, turbid reaction mixture turns into a clear solution within 30 seconds. Aliquots $(\sim 200 \mathrm{uL}$ were withdrawn after $1,2,3,4,5,7,10,15,20,30,40,50,60,75,90$, and $120 \mathrm{~min}$. To quench, these samples were added to $100 \mathrm{uL}$ of an aqueous solution of 2-dimethylaminoethanethiol hydrochloride $(20 \%)$. The clear colorless to slightly yellow solution was then filtered through a plug of silica-gel, and the silica-gel was washed with diethyl ether to give a total sample volume of $\sim 2 \mathrm{~mL}$. This sample was injected into the GC three times.

initial rate $=0.0048$

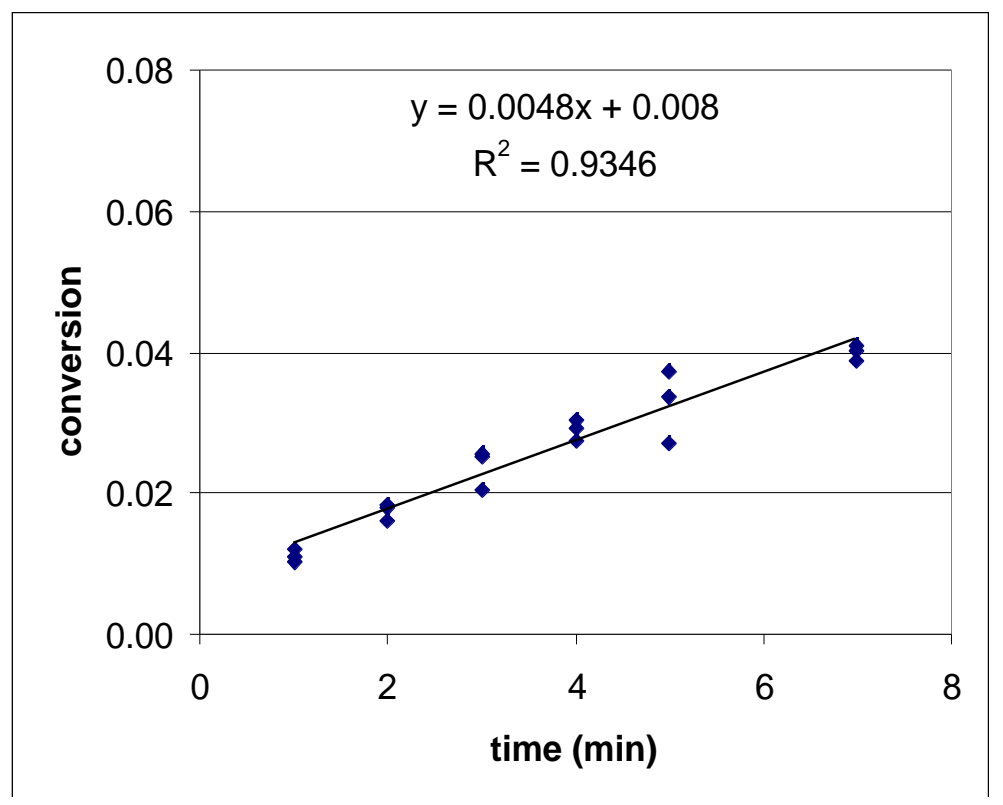




\begin{tabular}{|c|c|c|c|}
\hline time $(\min )$ & area $\mathrm{A}$ & area 16 & conversion \\
\hline 1 & 12860 & 631 & 0.012 \\
\hline 1 & 12372 & 552 & 0.011 \\
\hline 1 & 12795 & 543 & 0.010 \\
\hline 2 & 34151 & 2512 & 0.018 \\
\hline 2 & 34287 & 2535 & 0.018 \\
\hline 2 & 9756 & 637 & 0.016 \\
\hline 3 & 13355 & 1365 & 0.025 \\
\hline 3 & 11101 & 928 & 0.020 \\
\hline 3 & 10946 & 1148 & 0.026 \\
\hline 4 & 27766 & 3328 & 0.029 \\
\hline 4 & 12078 & 1487 & 0.030 \\
\hline 4 & 10840 & 1212 & 0.027 \\
\hline 5 & 11072 & 1226 & 0.027 \\
\hline 5 & 8993 & 1370 & 0.037 \\
\hline 5 & 9399 & 1287 & 0.034 \\
\hline 7 & 10935 & 1832 & 0.041 \\
\hline 7 & 11552 & 1828 & 0.039 \\
\hline 7 & 11009 & 1802 & 0.040 \\
\hline 10 & 12854 & 2468 & 0.047 \\
\hline 10 & 14426 & 2433 & 0.041 \\
\hline 10 & 13725 & 2682 & 0.048 \\
\hline 15 & 11317 & 2358 & 0.051 \\
\hline 15 & 12119 & 2963 & 0.060 \\
\hline 15 & 12566 & 3181 & 0.062 \\
\hline 20 & 11812 & 2441 & 0.051 \\
\hline 20 & 12436 & 3183 & 0.063 \\
\hline 20 & 11842 & 3323 & 0.069 \\
\hline 30 & 12211 & 3588 & 0.072 \\
\hline 30 & 11462 & 3957 & 0.085 \\
\hline 30 & 11221 & 3476 & 0.076 \\
\hline 40 & 16440 & 6042 & 0.090 \\
\hline 40 & 14900 & 4867 & 0.080 \\
\hline 40 & 14597 & 4690 & 0.079 \\
\hline 50 & 13427 & 5485 & 0.100 \\
\hline 50 & 13921 & 6294 & 0.111 \\
\hline 50 & 13363 & 5018 & 0.092 \\
\hline 60 & 7889 & 3343 & 0.104 \\
\hline 60 & 21281 & 9173 & 0.106 \\
\hline 60 & 8399 & 3592 & 0.105 \\
\hline 75 & 12760 & 6568 & 0.126 \\
\hline 75 & 13367 & 7408 & 0.136 \\
\hline 75 & 13114 & 7226 & 0.135 \\
\hline 90 & 10632 & 6611 & 0.152 \\
\hline 90 & 11831 & 7960 & 0.165 \\
\hline 90 & 10616 & 7031 & 0.162 \\
\hline 120 & 8937 & 6936 & 0.190 \\
\hline 120 & 19728 & 13718 & 0.170 \\
\hline 120 & 9138 & 6643 & 0.178 \\
\hline
\end{tabular}




\section{References}

(1) Denmark, S. E.; Wehrli, D. Org. Lett. 2000, 2, 565.

(2) Denmark, S. E.; Choi, J.-Y.; J. Am. Chem. Soc 1999, 121, 5821.

(3) Kropp, P. J., Crawford, S. D. J. Org. Chem. 1994, 59, 3102.

(4) Ukai, T.; Kawazura, H.; Ishii, Y.; Bonnett, J. J.; Ibers, J. A. J. Organomet. Chem. 1974, $65,253$. 